\title{
Molecules in strong laser fields
}

In depth study of $\mathrm{H}_{2}$ molecule

DISSERTATION

zur Erlangung des akademischen Grades

\author{
Dr. Rer. Nat. \\ im Fach Physik \\ eingereicht an der \\ Mathematisch-Wissenschaftlichen Fakultät I \\ Humboldt-Universität zu Berlin \\ von \\ M.Sc. Manohar Awasthi \\ geboren am 03.12.1978 in Kanpur, Indien
}

Präsident der Humboldt-Universität zu Berlin:

Prof. Dr. Dr. h.c. Christoph Markschies

Dekan der Mathematisch-Wissenschaftlichen Fakultät I:

Prof. Dr. Lutz-Helmut Schön

Gutachter:

1. P.D. Dr. Alejandro Saenz

2. Prof. Dr. Jörn Manz

3. Prof. Dr. Manfred Lein

eingereicht am: 31.08.2009

Tag der mündlichen Prüfung: 29.10.2009 


\begin{abstract}
A method for solving the time-dependent Schrödinger equation (TDSE) describing the electronic motion of the molecules exposed to very short intense laser pulses has been developed. The time-dependent electronic wavefunction is expanded in terms of a superposition of field-free eigenstates. The field-free eigenstates are calculated in two ways. In the first approach, which is applicable to two electron systems like $\mathrm{H}_{2}$, fully correlated field-free eigenstates are obtained in complete dimensionality using configuration-interaction calculation where the one-electron basis functions are built from $B$ splines. In the second approach, which is even applicable to larger molecules, the field-free eigenstates are calculated within the single-active-electron (SAE) approximation using density functional theory. In general, the method can be divided into two parts, in the first part the field-free eigenstates are calculated and then in the second part a time propagation for the laser pulse parameters is performed.
\end{abstract}

The $\mathrm{H}_{2}$ molecule is the testing ground for the implementation of both the methods. The reliability of the configuration interaction (CI) based method for the solution of TDSE (CI-TDSE) is tested by comparing results in the low-intensity regime to the prediction of lowest-order perturbation theory. Another test for the CI-TDSE method is in the united atom limit for the $\mathrm{H}_{2}$ molecule. By selecting a very small value of the internuclear distance close to zero for the $\mathrm{H}_{2}$ molecule, Helium atom is obtained. The results in this united-atom limit are compared to the existing results for Helium atom. Once the functionality and the reliability of the method is established, it is used for obtaining accurate results for molecular hydrogen exposed to intense laser fields. The results for the standard $800 \mathrm{~nm}$ Titanium-Sapphire laser and its harmonics at $400 \mathrm{~nm}$ and $266 \mathrm{~nm}$ are shown. The results for a scan over a wide range of incident photon energies as well as dependence on the internuclear distance are presented. The photoelectron spectra including above-threshold-ionization peaks is also demonstrated.

The CI-TDSE results for $\mathrm{H}_{2}$ are used for testing the validity of SAE approximation. In strong field physics, there are models based on the SAE approximation. Most popular are the Ammosov-Delone-Krainov (ADK) model, a molecular version of the ADK model called MO-ADK (MO stands for molecular orbital) and the strong field approximation (SFA). The validity of the second method for the solution of TDSE in SAE approximation is investigated by applying it to $\mathrm{H}_{2}$ molecule where the exact two-electron results were already calculated using CI-TDSE. The SAE method uses density-functional-theory (DFT) for the description of field-free eigenstates and is thus abbreviated as DFT-SAE-TDSE. Since DFT is used for the calculation of field-free states, different functionals were also tested. The validity of MO-ADK model is also investigated.

After establishing the DFT-SAE-TDSE method, the first excited state $\mathrm{B}^{1} \Sigma_{u}^{+}$of $\mathrm{H}_{2}$ is studied over a large range of laser parameters. When compared to the ground state, the $\mathrm{B}^{1} \Sigma_{u}^{+}$state is closely spaced to other excited states. The effect of the closely lying excited states on ionization and excitation is studied. The earlier predictions made by the $a b$ initio calculations in the quasi-static regime are investigated. The results for different approaches based on SAE approximation are also compared.

After successful testing of DFT-SAE-TDSE method on $\mathrm{H}_{2}$ molecule, the results for larger molecules like $\mathrm{N}_{2}, \mathrm{O}_{2}$ and $\mathrm{C}_{2} \mathrm{H}_{2}$ in the DFT-SAE framework are presented. 
The applicability of DFT-SAE-TDSE is not limited to just linear molecules. It has been applied to water molecule and the study is already underway. The DFT-SAETDSE method has a lot potential and promises to be a method for the theoretical treatment for larger molecular systems in future. 



\section{Zusammenfassung}

Eine Methode zur Lösung der zeitabhängigen Schrödingergleichung (engl. timedependent Schrödinger equation, TDSE) wurde entwickelt, welche das Verhalten der Elektronenbewegung in Molekülen beschreibt, die ultrakurzen, intensiven Laserpulsen ausgesetzt werden. Die zeitabhängigen elektronischen Wellenfunktionen werden durch eine Superposition von feldfreien Eigenzuständen beschrieben, welche auf zwei Weisen berechnet werden. Im ersten Ansatz, welcher auf ZweielektronenSysteme wie $\mathrm{H}_{2}$ anwendbar ist, werden die voll korrelierten feldfreien Eigenzustände in voller Dimensionalität in einem Konfigurations-Wechselwirkungs Verfahren (engl. configuration interaction, CI) bestimmt, wobei die Einelektron-Basisfunktionen mit $B$-Splines beschrieben werden. Im zweiten Verfahren, welches sogar auf größere Moleküle anwendbar ist, werden die feldfreien Eigenzustände in der Näherung eines aktiven Elektrons (engl. single active electron, SAE) mit Verwendung der Dichtefunktionaltheorie (DFT) bestimmt. Im Allgemeinen kann die Methode zum Auffinden der zeitabhängigen Lösung in zwei Schritte, dem Auffinden der feldfreien Eigenzustände und einer Zeitpropagation in Abhängigkeit der Laserpuls-Parameter, unterteilt werden.

Das $\mathrm{H}_{2}$ Molekül wird als Testfall für die Implementation beider Methoden verwendet. Die Zuverlässigkeit der CI basierten Methode für die Lösung der TDSE (CI-TDSE) wird für schwache Laserintensitäten durch den Vergleich mit der Störungstheorie niedrigster Ordnung untersucht. Ein weiterer Test besteht in der Betrachtung des Übergangs zu kleinen Kernabständen. Durch Verwendung von Kernabstände nahe Null führt man das $\mathrm{H}_{2}$ Molekül auf ein Heliumatom zurück, sodass die berechneten Ergebnisse mit vorhandenen Daten von Helium verglichen werden können. Nachdem die Funktionalität und Zuverlässigkeit der Methode überprüft ist, wird sie verwendet, um präzise Resultate für molekularen Wasserstoff in intensiven Laserfeldern zu erhalten. Die Ergebnisse für einen 800 nm Titan:Saphir Laser und seinen Harmonischen bei $400 \mathrm{~nm}$ und $266 \mathrm{~nm}$ werden präsentiert. Des weiteren werden Ergebnisse eines Scanns über einen großen Bereich von Energien der einfallenden Photonen und einer Variation des Kernabstand gezeigt. Die Photoelektronenspektren enthalten Peaks über der Ionisationsschwelle, welche ebenfalls vorgestellt werden.

Die CI-TDSE Ergebnisse für $\mathrm{H}_{2}$ werden verwendet, um die Gültigkeit der SAE Näherung zu überprüfen. In der Physik starker Felder existieren Modelle, die auf der SAE Näherung basieren. Zu den bekanntesten zählen das Ammosov-Delone-Krainov (ADK) Modell, eine molekulare Version des ADK Modells namens MO-ADK (MO steht für Molekülorbital) und die Starkfeld-Näherung (engl. strong field approximation, SFA). Die Gültigkeit des zweiten Modells für die TDSE Lösung in SAE Näherung wird untersucht, indem sie auf das $\mathrm{H}_{2}$ Molekül angewandt wird, dessen exakte Zweielektronen-Resultate bereits mittels CI-TDSE ermittelt wurden. Da die SAE Methode DFT für die Beschreibung der feldfreien Eigenzustände verwendet, wird Sie mit DFT-SAE-TDSE abgekürzt. Verschiedene Funktionale im Rahmen der DFT werden untersucht. Ebenso wird die Gültigkeit des MO-ADK Modells überprüft.

Nach der Implementation der DFT-SAE-TDSE Methode, wird der erste angeregte Zustand $B^{1} \Sigma_{u}^{+}$von $\mathrm{H}_{2}$ für einen großen Bereich unterschiedlicher Laserparameter untersucht. Im Vergleich zum Grundzustand liegt der $B^{1} \Sigma_{u}^{+}$Zustand nahe an anderen angeregten Zuständen. Deren Einfluss auf die Ionisation und Anregung wird 
ebenfalls erforscht. Die früheren Vorhersagen durch $a \mathrm{~b}$ initio Rechnungen im quasistatischen Regime werden untersucht. Die Resultate der unterschiedlichen auf der SAE Näherung basierenden Ansätze werden verglichen.

Nach erfolgreichem Testen der DFT-SAE-TDSE Methode für das $\mathrm{H}_{2}$ Molekül werden größere Moleküle wie $\mathrm{N}_{2}, \mathrm{O}_{2}$ und $\mathrm{C}_{2} \mathrm{H}_{2}$ im Rahmen der DFT-SAE Näherung behandelt. Die Anwendbarkeit der DFT-SAE-TDSE Methode ist nicht auf lineare Moleküle beschränkt. Sie wurde auf das Wassermolekül angewendet und eine genauere Untersuchung ist bereits im Gange. Die DFT-SAE-TDSE Methode hat viel Potenzial und verspricht in Zukunft Verwendung bei der theoretischen Behandlung größerer Moleküle zu finden. 


\section{Contents}

1 Introduction 1

2 Time-dependent Schrödinger equation $\quad 11$

2.1 One-electron Schrödinger equation . . . . . . . . . . . . . . . 12

2.2 Two-electron Schrödinger equation . . . . . . . . . . . . . . 15

2.3 Time propagation . . . . . . . . . . . . . . . . 17

3 SAE-TDSE 21

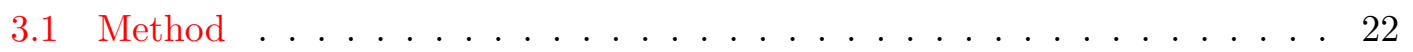

3.2 Computational details . . . . . . . . . . . . . . . . 25

4 Models and approximations in strong fields physics $\quad 29$

4.1 ADK Model . . . . . . . . . . . . . . . . . . . . . 30

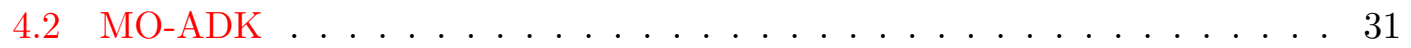

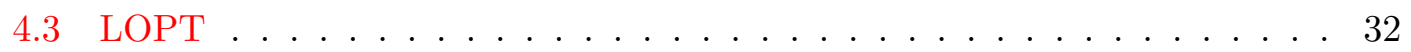

5 CI-TDSE works $\mathbf{3 5}$

5.1 He: United atom limit . . . . . . . . . . . . . . . . . . 35

5.2 LOPT for $\mathrm{He} \ldots \ldots \ldots \ldots \ldots \ldots$

6 CI-TDSE results for molecular hydrogen $\quad 41$

6.1 Photon energy scan . . . . . . . . . . . . . . . . . . . 41

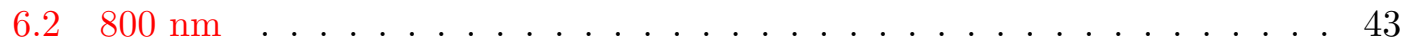

6.2.1 Variation of internuclear distance . . . . . . . . . . . . . 44

$6.3400 \mathrm{~nm} \ldots \ldots \ldots \ldots \ldots \ldots \ldots \ldots$

6.3.1 Variation of internuclear distance . . . . . . . . . . . . . . 48

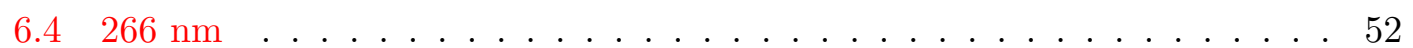

6.4.1 Variation of internuclear distance . . . . . . . . . . . . . 52

6.5 Photoelectron spectra . . . . . . . . . . . . . . . 54

7 SAE-TDSE Results $\quad \mathbf{5 7}$

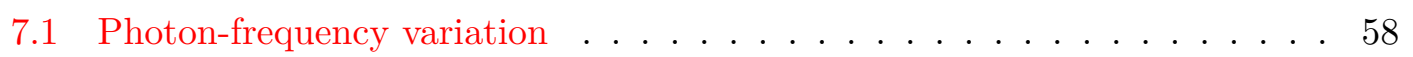

7.2 Intensity variation $\ldots \ldots \ldots \ldots \ldots \ldots \ldots$

7.3 Comparison to MO-ADK . . . . . . . . . . . . . . 68

8 First excited state $\quad \mathbf{7 3}$

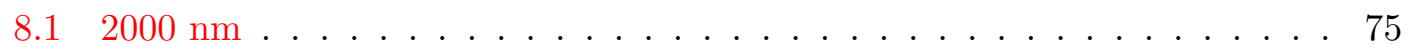




\section{Contents}

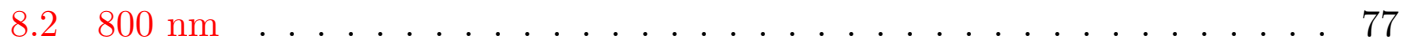

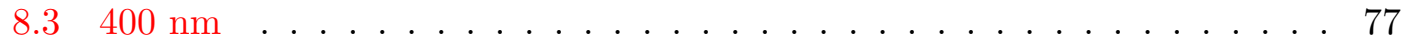

8.4 Single photon ionization . . . . . . . . . . . . . . . . . 78

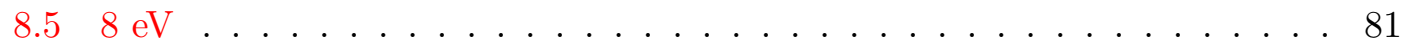

$8.6 R=1.4$ a.u. . . . . . . . . . . . . . 84

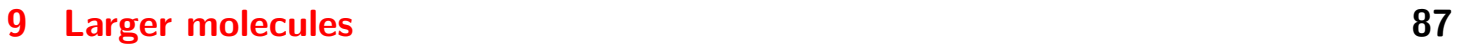

9.1 Photon energy scan . . . . . . . . . . . . . . . . . . . . . . 89

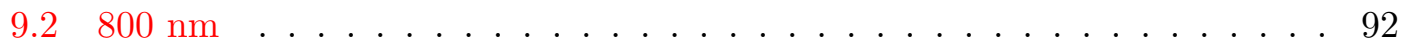

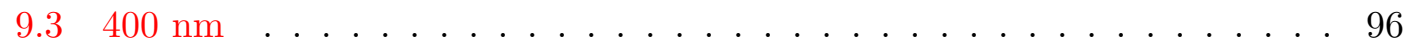

$\begin{array}{lr}10 \text { Summary } & 99\end{array}$

$\begin{array}{ll}\text { Abbreviations } & 103\end{array}$

$\begin{array}{lr}\text { Acknowledgments } & 105\end{array}$ 


\section{Introduction}

The sensational development of new sources of electromagnetic radiation has generated a considerable amount of interest in the interaction processes between matter and radiation. These new sources include the free-electron lasers (FEL) which can generate high energy photons in the extreme ultraviolet (XUV) or soft X-ray region, attosecond pulses obtained from the high order harmonic generation (HOHG) and table-top terawatt lasers [1]. These new sources have revolutionized the field and led to more precise understanding of structure and dynamics of atoms and molecules. The investigation of the structure of matter and observation of its temporal evolution under perturbations are fundamental problems in physics and chemistry. A recent advancement in this direction was the tomographic imaging of the electronic ground state wavefunction of the nitrogen molecule [2]. Such experiments may have a great impact on the way physics and chemistry will be taught. The orbitals and wavefunctions are no longer theoretical concepts used in physics and chemistry, they can now be imaged using tomography.

These days, high intensity lasers with a pulse duration of few femtoseconds are commercially available. The laser pulses can be generated with total control over the absolute phase. The absolute phase of the electric field and the carried envelope in a laser pulse can be controlled [3-5] and this can be applied to study the dissociation dynamics of molecules [6] or non-sequential double ionization in $\mathrm{H}_{2}$ [7]. The generation of single cycle pulses using mixing of waves [8] is now possible. Wave-mixing is purely an optical method and produces a single pulse of around $1.6 \mathrm{fs}$. The high order harmonic generation can be used to produce attosecond pulses [9, 10]. A single attosecond pulse of around 130 as [11] can now be produced.

The experimental schemes like recoil ion momentum spectroscopy $[12,13]$ and its advanced version popularly known as COLTRIMS (Cold Target Recoil Ion Momentum Spectroscopy) [14-18] have provided great insight into the processes taking place in strong fields. The conventional pump-probe schemes $[19,20]$ have also contributed significantly in studying the nuclear dynamics of molecules exposed to electromagnetic fields [21-23].

Along with experimental progress, the theory of laser-atom and laser-molecule interaction also developed. Keldysh divided the field of laser-atom or laser-molecule interaction into two broad regimes [24]. This division was based on very simple parameters like field amplitude, frequency and binding energy of the system. A free electron in a laser field makes an oscillating motion at the frequency of the laser. The quiver energy or ponderomotive energy is given by

$$
U_{\mathrm{p}}=\left(\frac{F_{0}}{2 \omega}\right)^{2}
$$




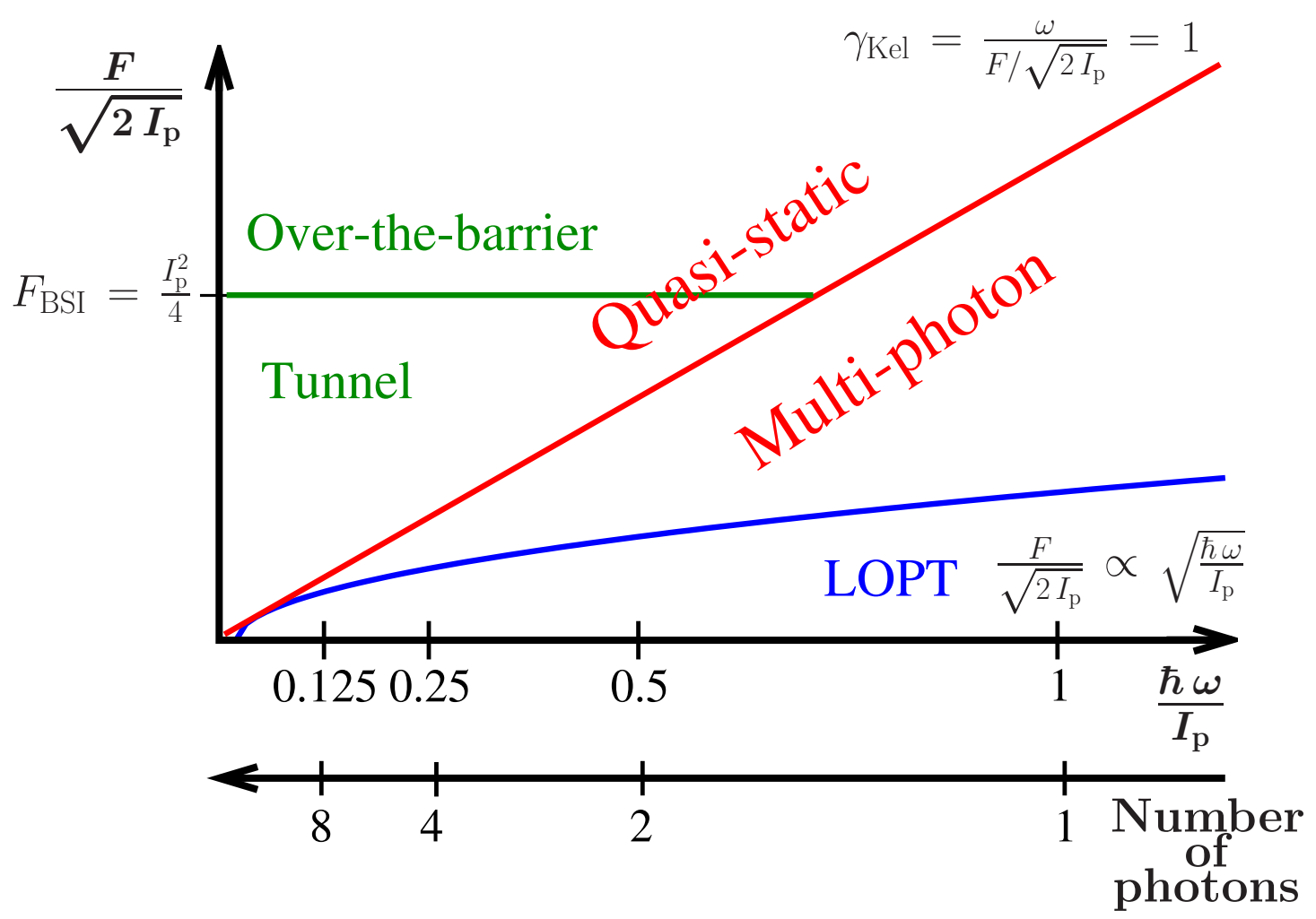

Figure 1.1: A schematic representation of the ionization regimes. Scaled field strength as a function scaled photon energy is shown. The solid red line shows $\gamma_{\mathrm{Kel}}=1$, which divides the region into multi-photon and quasi-static regimes. The quasi-static regime is further divided by the solid green line into tunnel and over-the-barrier ionization regimes. The region below the solid blue line is the region where LOPT can be applied.

where $F_{0}$ and $\omega$ are the amplitude and the angular frequency of the laser field, respectively. The Keldysh parameter $\left(\gamma_{\mathrm{Kel}}\right)$ is proportional to the ratio between the binding energy, $I_{\mathrm{p}}$, of the electron and the ponderomotive energy. It is defined as

$$
\gamma_{\text {Kel }}=\sqrt{\frac{I_{\mathrm{p}}}{2 U_{\mathrm{p}}}} .
$$

Depending on the Keldysh parameter the ionization process can be divided into regimes of multi-photon $\left(\gamma_{\text {Kel }} \gg 1\right)$ and quasi-static ionization $\left(\gamma_{\text {Kel }} \ll 1\right)$. Figure 1.1 shows a schematic division of ionization regimes over a range of parameters like field amplitude, field frequency and the binding energy of the system.

The quasi-static regime can be further divided into tunnel ionization and over-thebarrier ionization. Figure 1.2 shows a schematic depiction of tunnel and over-the-barrier ionization. The black curve is the field-free Coulomb potential with the ground state shown by thick solid maroon line. When the field strength is sufficiently high, the 
potential is modified to the red dashed curve. At this point the electron sees a very small potential and can tunnel through it, as shown by the green arrow. This is called tunnel ionization. When the field strength is further increased, as shown by blue dotted lines, the electron can simply escape over the barrier. This is called over-the-barrier ionization or barrier-suppression ionization (BSI). The field strength above which barrier suppression ionization occurs is $F_{\mathrm{B} S I}=I_{\mathrm{p}}{ }^{2} / 4$ is also shown in Figure 1.1. Further details of tunnel ionization are shown in Section 4.1.

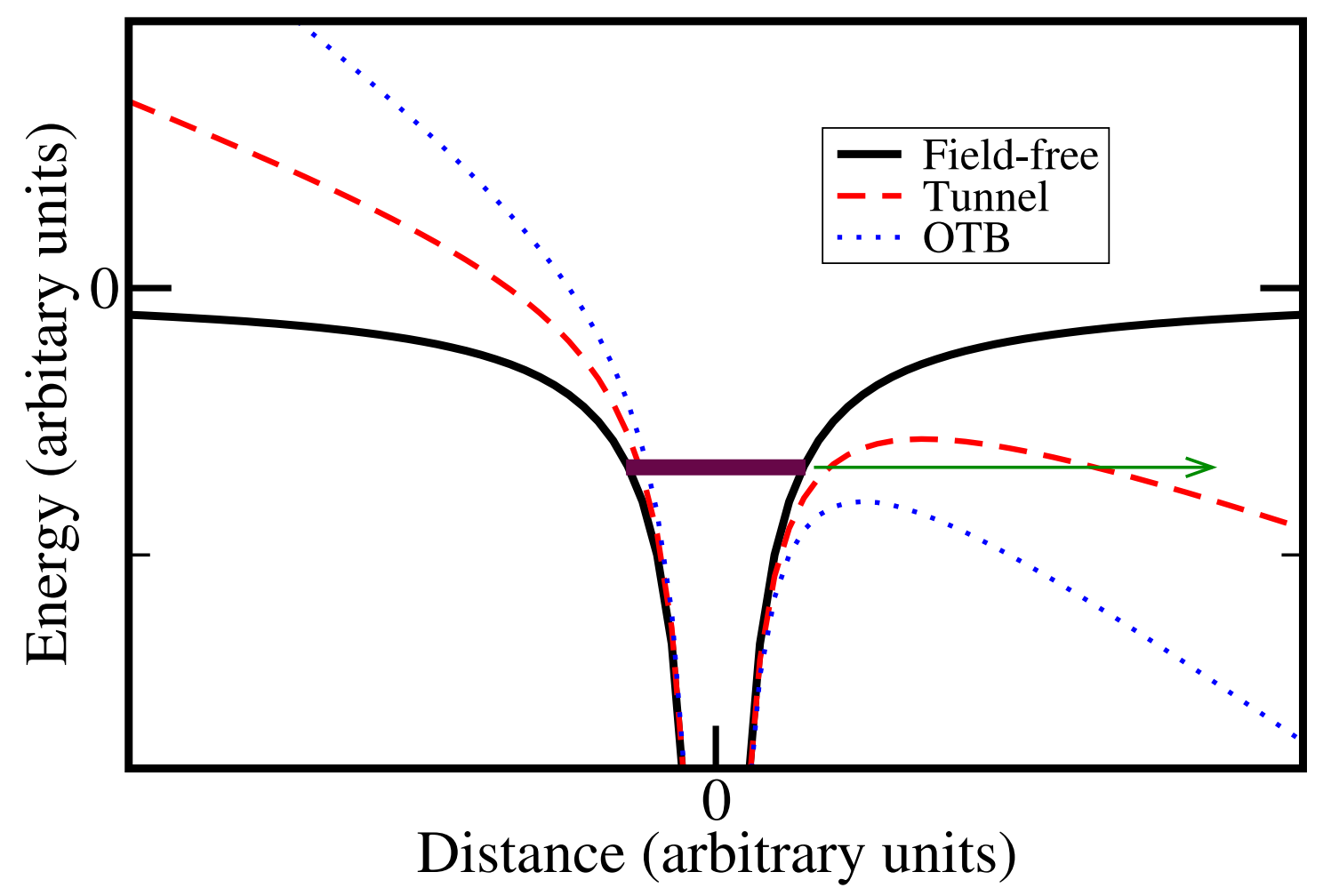

Figure 1.2: A schematic representation of tunnel and over-the-barrier ionization. The black curves represents the field-free Coulomb potential. The Coulomb potential is modified on the application of field and is shown by the dashed red curve. The process of tunneling is shown by the green arrow. The dotted blue curve shows over-the-barrier ionization. In this case the external field so strong that it suppresses the Coulomb potential completely.

In the multi-photon regime, the number of photons needed for ionization is relatively small compared to quasi-static regime and the ponderomotive energy is also low. When the intensity of the field is not very high, lowest-order perturbation theory (LOPT) is used to calculate the ionization rate. The LOPT is discussed lated in Section 4.3.

The initial investigations on simple atomic systems led to the discovery of interesting processes like high order harmonic generation (HOHG) [25-28], above threshold ionization (ATI) [29-32] or stabilization against ionization [33-36] have been found. The first 


\section{Introduction}

effect, HOHG, is coherent emission of the photons with shorter wavelength than the incident photons. It is the non-linear response of the atom to the intense laser field by emitting photons with integer multiples of incident laser frequency. The second effect, ATI, means that electrons absorb more photons than necessary for ionization while the third is the stabilization of the atom against ionization at sufficiently high intensities or photon frequencies.

In molecules, due to the additional degrees of freedom (vibrational and rotational motion), further effects occur. Some of the already discovered, well-known and interesting effects are :

Alignment The molecules tend to align themselves when exposed to electromagnetic fields. Initially this idea was only supposed to work for polar molecules where permanent dipole moment couples the molecule with the field and aligns it. Unfortunately, there are not many polar molecules. To extend the control of the spatial orientation toward a much broader class of molecules, Friedrich and Herschbach [37, 38] suggested to exploit the anisotropic polarizability interaction of an intense non-resonant laser field with the induced dipole moment of molecules. The interaction creates a potential minimum for the molecules along the polarization axis of the field, forcing them to liberate over a limited angular range instead of rotating freely with random spatial orientations. Since there is no interaction between the laser field and a possible permanent dipole moment, the molecules are aligned rather than oriented.

The interaction of an intense ultrashort laser pulse with molecules has theoretically been shown to create a superposition of coherently excited rotational states or a rotational wave packet [39-43]. This wave packet gives rise to transient alignment of molecules that is recurrent under field-free conditions. There has been much interest in this field-free alignment of molecules, because it provides a promising and versatile way to control molecules with an external field for a variety of applications (see review by Stapelfeldt and Seideman [44] and references therein). The revival structure in the field-free molecular alignment was first observed with Coulomb explosion imaging by Rosca-Pruna and Vrakking [45, 46]. The fundamental behavior and dynamics of the alignment have been extensively studied so far using Coulomb explosion imaging [47, 48] and polarization spectroscopy [49-51].

Bond softening Bond softening or bond weakening may be observed in a molecule exposed to strong laser field. Intuition suggests that a sufficiently strong laser field can weaken molecular bonds and induce dissociation. This mechanism is known as bond softening. Most of the investigations of bond softening were carried out on $\mathrm{H}_{2}^{+}[52,53]$ or singly ionized molecular ions [54]. No matter how natural or intuitive it might seem, it was predicted by Hiskes in 1961 that pre-dissociation or bond softening will not occur in neutral homonuclear molecules which have ${ }^{1} \Sigma_{g}^{+}$ ground state [55]. It took almost 40 years to theoretically show that bond softening can occur in neutral homonuclear $\mathrm{H}_{2}$ molecule [56]. 
Stabilization against dissociation and bond hardening It was theoretically predicted that the higher lying vibrational states of $\mathrm{H}_{2}^{+}$molecule in a laser field will become stable against dissociation on increasing the laser intensity [53, 57-60]. This effect can be understood in terms of a light-induced potential well that creates light-induced bound states. These light-induced bound states are responsible for the stabilization of dissociation process. Two manifestations of bond hardening in $\mathrm{H}_{2}^{+}$near 1-photon [61] and 3-photon [62] resonances were inferred. They were received with great interest, as at that time the stabilization of the molecular bond was a candidate for a universal mechanism explaining the invariance of ion kinetic energies with changes of intensity and pulse duration [63, 64]. Later, it was established that this invariance is a signature of rapid, sequential ionization at the critical internuclear distance [65-67]. After that there was a surprising lack of clearcut confirmation of the bond hardening effect. Then there was some work casting doubt on the existence of light-induced bound states [68, 69], the idea of bond hardening had again become only a remote theoretical possibility until the experiments by Frasinski and coworkers put an end to all the scepticism [70, 71].

Above threshold dissociation Analogous to the atomic process of above threshold ionization, in molecules above threshold dissociation is observed, where the molecule absorbs more photons than necessary for the fragmentation [72-76]. With the advanced experimental techniques like 3 -D momentum imaging the experimentalists have separated the ionization and dissociation dynamics in $\mathrm{H}_{2}^{+}$[77]. Most of the studies on above threshold dissociation were based on $\mathrm{H}_{2}^{+}$, but it was only recently that higher order (more than three) above threshold dissociation was seen in $\mathrm{H}_{2}^{+}$ at $800 \mathrm{~nm}$ [78].

Enhanced ionization Molecules show very high ionization rates at internuclear distances that are larger than the equilibrium internuclear distance. This effect is called enhanced ionization [66, 67, 76, 79-81]. It was explained on the basis of charge resonance enhanced ionization (CREI) in $\mathrm{H}_{2}^{+}$[82, 83]. The experimental finding that the kinetic energy of ionized fragments is smaller than the expected for a Coulomb explosion from the equilibrium internuclear distance was explained by enhanced ionization $[63,65,84,85]$. Initially it was thought that enhanced ionization will occur for molecular ions only, but again it was shown that it can occur in neutral molecules like $\mathrm{H}_{2}[56,86]$. There are still questions regarding enhanced ionization which need to be answered. One of them is enhanced ionization in $\mathrm{H}_{2}^{+}$. Zuo and Bandrauk [67] had predicted two peaks for enhanced ionization at an internuclear distance of $7 a_{0}$ and $10 a_{0}$ in $\mathrm{H}_{2}^{+}$. Their results were obtained by solving two-dimensional TDSE in fixed-nuclei approximation. The experiments by Gibson et. al [69] had confirmed their prediction, but a recent experiment shows only a single peak with the explanation that nuclear motion averages over the two peaks obtained in fixed-nuclei approximation [87].

Interference in HOHG The HOHG also occurs in molecules [88-90], but due to additional degrees of freedom in molecules, the harmonic spectra shows more fea- 


\section{Introduction}

tures [91-94]. One of the features is interference due to multiple nuclear centers in the molecules [95-99]. It has been theoretically found that the HOHG is also sensitive to the molecular orientation [93]. Most of the orientation dependence is due to an interference effect which can lead to a complete suppression of harmonics [92, 95]. The interference is simple in molecular hydrogen [92, 95] but more complicated in larger molecules due to the more complicated electronic structure [90]. Recently, it was shown for argon dimers that the momentum distribution of the photoelectrons exhibits interference due to the emission from the two atomic argon centers, in analogy with a Young's double-slit experiment [100].

The processes listed above are not complete, but some of the most basic processes that can take place when a molecule is exposed to a strong field. An understanding of these processes is essential for understanding further complicated processes of molecular dynamics when a molecule is exposed to a laser field. In order to completely understand the molecular dynamics in laser fields, the solution of time-dependent Schrödinger equation (TDSE) is incumbent. The strong field physics is heavily dependent on models, approximations and solution of TDSE in lower dimensions [101]. Even though these have been successful in explaining certain phenomenon, they are unable to provide a complete picture of the molecular dynamics in the laser field. The full solution of the TDSE for molecules exposed to laser fields is not an easy task. The complexity of the solution and efforts required to solve the TDSE increase as the molecular size increases. Using the solution of the full TDSE one can test these models, approximations and lower dimensional solutions. The solution of the TDSE can be used to set the limits within which all these approximations and models can be used.

Molecular hydrogen plays a prominent role for experiments on laser-matter interaction, because it is from the theoretical point of view the simplest molecular system that contains more than a single electron. Compared to $\mathrm{H}_{2}^{+}$(that in addition lacks the manyelectron aspect) $\mathrm{H}_{2}$ is also much easier to handle experimentally. Therefore, a plethora of experimental data exists for $\mathrm{H}_{2}$ exposed to laser fields (see [18] and references therein). Despite being almost the simplest molecule, attempts to describe the behaviour of $\mathrm{H}_{2}$ in short laser pulses in a non-simplified manner are very sparse. So far, practically all experiments were analyzed by assuming tunnel ionization. The corresponding ionization rates were obtained from simple atomic tunneling models like the Ammosov-DeloneKrainov (ADK) theory [102]. The regime of applicability of tunnel-ionization is shown in Figure 1.1 and details of ADK model are discussed in Section 4.1. Only recently an extension of the atomic ADK model incorporating the molecular structure (MO-ADK) was proposed and applied for the interpretation of experimental results [103, 104]. The MO-ADK model will be discussed in Section 4.2. An alternative approach has been proposed that is also based on the quasi-static approximation but adopts $a \mathrm{~b}$ initio static ionization rates and time-averaged field-distorted Born-Oppenheimer potentials. It was successfully used for predicting the vibrational final-state distribution of $\mathrm{H}_{2}^{+}$formed in laser-induced ionization of $\mathrm{H}_{2}$ [105]. Due to the good agreement between the molecular $a \mathrm{~b}$ initio results and the atomic ADK model in the relevant intensity regime, the ADK model was in fact used in the calculation for convenience reasons. 
The quasi-static approximations (either using simple tunnel-ionization models or $a \mathrm{~b}$ initio dc rates) are valid only for high intensities and low photon frequencies (see Figure 1.1). In the opposite limit (low intensity and high photon frequency) the ionization process may be described by lowest-order perturbation theory (LOPT). An advantage of LOPT is that it introduces well-defined approximations and can systematically be improved on. However, LOPT calculations are computationally demanding and thus the first systematic and correlated calculations of non-resonant LOPT ionization rates for $\mathrm{H}_{2}$ have only recently been performed [106]. The details of LOPT are discussed in Section 4.3.

Another popular approach is the Keldysh-Faisal-Reiss (KFR) theory, also known as strong-field approximation (SFA). This approach can alternatively be viewed as being the first term of an intense-field $S$-matrix theory (ISMT). While higher-order terms have been discussed for atoms [107], the application to molecules was so far limited to the first-order term. This corresponds to the traditional strong-field approximation in which the process is described as a transition from a field-free initial state to a final Volkov state ignoring thus the Coulomb interaction of the ejected electron with the remaining ion. Molecular effects have recently also been incorporated [108] into the model. However, there is some dispute about the use of length or velocity gauge and the applicability of a Coulomb-correction factor [109].

All the models discussed above are intrinsically adiabatic models (at least with respect to the electronic motion) and thus effects due to the shortness of ultrashort laser pulses are not taken into account. In addition, all models have their drawbacks even within their (assumed) range of applicability. While LOPT rates are comparatively difficult to calculate (requiring a summation/integration over in principle all intermediate fieldfree states), the most easily obtained ADK rate does not consider any photon-frequency dependence. The SFA calculation requires the evaluation of the field-free ground-state wavefunction and its overlap with the Volkov state. It incorporates some frequency dependence (especially channel-closing aspects), but does not contain any information on the possible influence of (intermediate) resonances. This is on the other hand considered in LOPT. Since a direct comparison with experimental data is often difficult and in addition requires usually an averaging over many parameters (the spatio-temporal shape of the laser pulse that may even not be known very precisely), a direct comparison to full solutions of the time-dependent Schrödinger equation (TDSE) is desirable. A further advantage of such a comparison is the ability to compare results at a well defined level of approximation. For example, the exact influence of nuclear motion (rotation and vibration) is still rather unclear (and depends on the experimental parameters like pulse length). While the verification of a model by means of a comparison to experimental results has to simulate the complete process, a theoretical comparison can be performed by concentrating on a single (artificially fixed) nuclear configuration relative to the laser polarization (and using identical laser-pulse parameters).

However, solving the TDSE even within the fixed-nuclei approximation is a very demanding task. Thus most of the solutions of the TDSE of molecules were restricted to the single-electron system $\mathrm{H}_{2}^{+}$, and even there often one or two-dimensional model Hamiltonians were applied. The same idea of reduced symmetry has also been utilized 


\section{Introduction}

for $\mathrm{H}_{2}$ [110] and is still popular, see, e.g., [111]. A full three-dimensional approach was introduced in [112-114]. A special grid-technique using the dual transformation of the electronic wavepacket allowed the efficient solution of the TDSE, but only for a parallel orientation of the laser polarization and the molecular axis (reducing the dimensionality due to cylindrical symmetry). An intrinsic problem of grid methods is the non-unique definition of the ionization yield that is usually encountered.

In this work an alternative approach for solving the TDSE describing molecular hydrogen exposed to short intense laser pulses was implemented. It is based on a spectral method in which the time-dependent electronic wave function is expanded in terms of a superposition of field-free eigenstates. The latter are the result of a configuration interaction (CI) calculation and thus include correlation. This sometimes called spectral approach (in contrast to grid techniques) has proven its capabilities in the context of atomic calculations (see review [115] and references therein) as well as recently also for $\mathrm{H}_{2}^{+}$[116]. The details of the method are described in Chapter 2. Although a variation of the internuclear distance and even a different orientation of the molecule with respect to the polarization of the laser field can be considered with the present method almost without modifications [117], the present work concentrates mainly on results obtained for a fixed internuclear distance and a parallel orientation of a linearly polarized laser field and the molecular axis. The correctness of the numerical implementation will be shown in Chapter 5. This is done by comparing for very small internuclear distances to helium results and for low-intensities to LOPT predictions. The results for $\mathrm{H}_{2}$ will be shown and discussed in Chapter 6. The validity of the single-active electron approximation (SAE) is also discussed. The SAE approximation is important, since it may be a valuable approach for extending the method to systems with more than two electrons while pertaining the explicit time-dependence, as has been done for atoms. The ability of obtaining photoelectron spectra (including above-threshold ionization ATI) is also shown. The results obtained by this method were further verified by the group of Fernando Martin [118].

After successful implementation of the spectral method for the $\mathrm{H}_{2}$ molecule, the goal was set for the treatment of larger molecules. The exact treatment of larger molecules with many electrons is very difficult. So a method for the solution of TDSE for molecules in general using density functional theory (DFT) in single-active-electron (SAE) approximation in frozen nuclei configuration was developed. This work was a part of a collaboration between three groups, namely Piero Decleva from University of Trieste, Alberto Castro from Free University of Berlin and our group at Humboldt University in Berlin. The method is described in Chapter 3. The initial testing of the method was done on $\mathrm{H}_{2}$ molecule where accurate results already exist. The details of DFT-SAETDSE method are discussed in Chapter 3. The results of TDSE for $\mathrm{H}_{2}$ molecule using DFT-SAE are shown in Chapter 7. The results for $\mathrm{H}_{2}$ molecule are used for testing the SAE approximation and existing SAE models. In Chapter 4 some common models like ADK, MO-ADK and LOPT used in strong field physics are described.

In Chapter 8 result of TDSE when starting with the first excited states, $\mathrm{B}^{1} \Sigma_{u}^{+}$, of $\mathrm{H}_{2}$ will be shown. The investigation of the $\mathrm{B}^{1} \Sigma_{u}^{+}$state of $\mathrm{H}_{2}$ was motivated by the results obtained by Manz and coworkers for the $\mathrm{B}^{1} \Sigma_{u}^{+}$state within the SAE approximation [119]. 
Finally in Chapter 9 the results for some larger molecules like $\mathrm{N}_{2}, \mathrm{O}_{2}$ and $\mathrm{C}_{2} \mathrm{H}_{2}$ are shown. Thus demonstrating the capability of the DFT-SAE method for the treatment of larger systems. 



\section{Time-dependent Schrödinger equation}

The time-dependent Schrödinger equation (TDSE) for diatomic molecules with two or effectively two electrons can be solved in three steps. In the first step, the field-free oneelectron Schrödinger equation for the diatomic molecular ion (like $\mathrm{H}_{2}^{+}, \mathrm{Li}_{2}^{+}, \mathrm{Na}_{2}^{+}, \mathrm{HeH}^{2+}$ ) is solved. For the case of effective one-electron systems, like alkali dimer ions $\left(\mathrm{Li}_{2}^{+}, \mathrm{Na}_{2}^{+}\right.$ etc.), the solution of one-electron Schrödinger equation is obtained by using a model potential. In the second step, a configuration interaction (CI) calculation is performed using the one-electron orbitals. The CI calculation gives the two-electron wavefunctions for the molecule. Finally, in the third step, a time-propagation is performed in the basis of field-free states obtained from the configuration interaction calculation. This method shall be referred as CI-TDSE.

The already existing ab initio codes for diatomic molecules may be classified as explicitly correlated (geminal) methods or configuration interaction (CI). In the former case the basis functions contain some functional form of the inter-electronic coordinate explicitly. The James-Coolidge type basis functions of Wolniewicz and co-workers have already a long time ago succeeded in providing tremendously accurate potential curves for the low-lying states of $\mathrm{H}_{2}$, especially its ground state [120]. A problem with such an approach is the numerical linear dependency of the basis sets. The numerical linear dependencies make convergence studies very difficult. It is possible to accurately calculate individual states but the but an accurate description of a large number of states using a single basis set is very tough. The bottleneck of all these calculations is the two-electron integral. The Gaussian geminals simplify such integrals and have been used for small molecular systems [121].

The configuration interaction can be performed using Gaussians (widely used in quantum chemistry codes). These codes are multipurpose and are not symmetry adapted for diatomic molecules, thus spend a lot of effort on optimization and accuracy. The advantage of these methods is that they can treat multiple electrons efficiently. The codes specific to diatomic molecules use elliptic (prolate-spheroidal) coordinates. The use of prolate-spheroidal coordinates automatically accounts for the electron-nucleus Coulombic cusp condition and tremendously reduces the amount of basis sets needed. The Gaussian based multipurpose codes have to use a large basis in order to implement the Coulombic cusp condition for diatomic molecules.

The methods discussed so far are based on global basis functions, i.e. basis functions span the complete position space. The global nature of functions helps to tackle larger molecular systems but has the disadvantage of the linear-dependency problem which makes systematic convergence study difficult. Another problem with such an approach is the calculation of Rydberg states and the density of continuum states. The calculation of specific amount of Rydberg states or a dense continuum requires enormous 


\section{Time-dependent Schrödinger equation}

computational effort. The evident solution to this problem is provided by local basis functions. In atomic electronic structure calculations $B$ splines have proven to combine locality with a very high degree of flexibility [122]. This flexibility allows a very good description of any type of wavefunction, both bound and continuum ones. The locality avoids the problem of linear dependencies. The inherent box discretization provided by the local basis leads to a well-defined discretization of the electronic continuum. With a given box size only those states that have a node at the box boundary appear in the calculation. The variation of the box size thus offers a tuning knob that determines the number of Rydberg states and the density of continuum states. This not only provides the ability to calculate bound to continuum transitions within the same basis set, but is also very practical when calculating quantities using a summation over complete sets of eigenstates, as is required in the calculation of perturbative expressions.

At the start of this work, the framework for the solution of field-free two-electron Schrödinger already existed in the group [123]. The basic steps for the solution of the field-free two-electron Schrödinger equation are shown in Section 2.1 and 2.2. The method could successfully and accurately calculate the various states of $\mathrm{H}_{2}$, including the doubly excited states. The main goal of this work was to efficiently solve the TDSE for $\mathrm{H}_{2}$ molecule in the basis of field-free $\mathrm{H}_{2}$ states (spectral method). The expansion of time-dependent wavefunction (in field wavefunction) in terms of field-free states leads to coupled differential equations which can be solved by a partial differential equation solver. The TDSE for $\mathrm{H}_{2}$ molecule exposed to a laser field can also be solved on grid and was already done by Kono and co-workers [112-114]. The grid based method suffer from a drawback that the ionization process is not uniquely defined. The ionization depends on the grid boundary conditions,i.e. absorbing boundary conditions at the grid boundaries. In the spectral method the ionization is uniquely defined, it is sum of the population of all the states above the ionization threshold. Another advantage of the spectral method is that once a converged basis set is obtained, only time-propagation is to be performed for different laser parameters. Whereas in grid methods, the complete process of solution of TDSE has to be started from zero for a set of new laser parameters. This work is the first attempt to solve the TDSE for $\mathrm{H}_{2}$ molecule in full dimensionality using the spectral method and during the course of this thesis, the results were also verified by Fernando Martín's group who used a similar approach for the solution of TDSE [118] for $\mathrm{H}_{2}$. The details of time-propagation in the basis of field-free states are shown in Section 2.3.

\subsection{One-electron Schrödinger equation}

The one-electron Schrödinger equation (OESE) for a diatomic molecule is solved in prolate spheroidal coordinates within the Born-Oppenheimer approximation. The OESE is solved by placing the diatomic molecule in a finite volume or a box. In the Figure 2.1, a schematic diagram shows the description of the system. The nuclei $A$ and $B$ are separated by the fixed internuclear distance $R$. The electron is at a distance of $r_{A}\left(r_{B}\right)$ from nuclei $A(B)$. The coordinate transformation to prolate spheroidal coordinates 
$(\xi \in[1, \infty) ; \eta \in[-1,1] ; \phi \in[0,2 \pi])$ is done in the following manner,

$$
\xi=\frac{r_{A}+r_{B}}{R} \text { and } \eta=\frac{r_{A}-r_{B}}{R} .
$$

$\phi$ is the angle around the internuclear axis. It is assumed that the one-electron Hamilto-

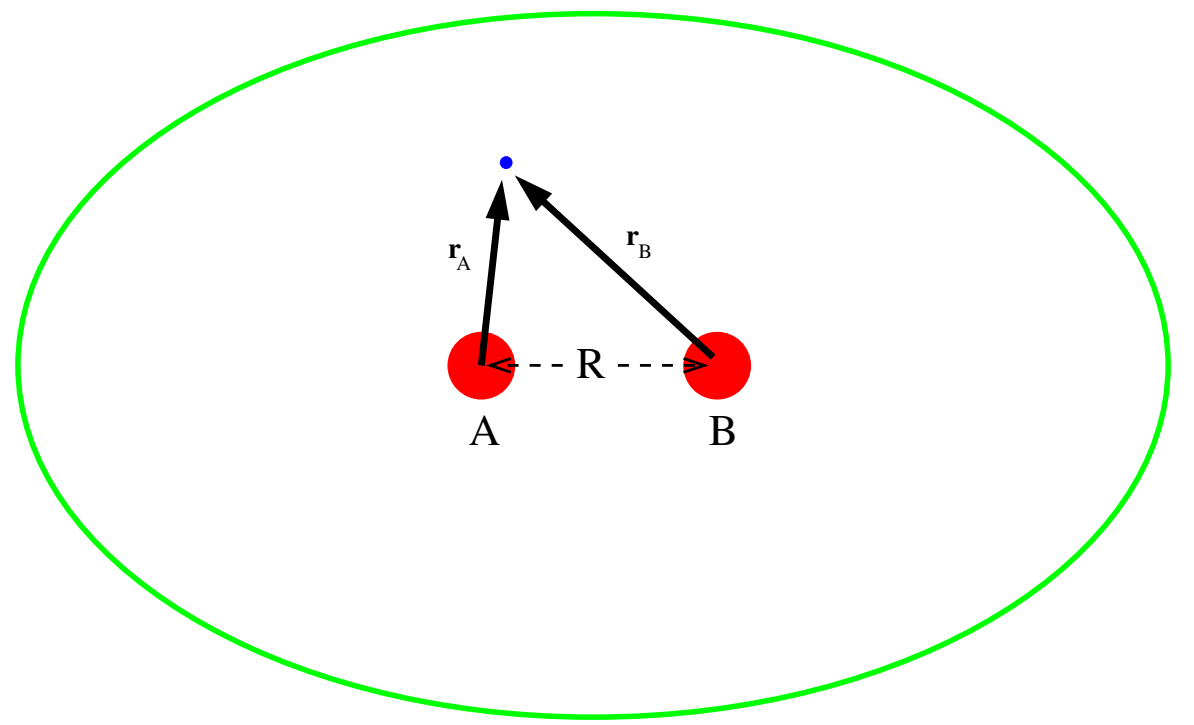

Figure 2.1: A schematic representation of (effective) one-electron system. The green boundary represents the enclosing box.

nian $\hat{\mathrm{h}}$ possesses rotational symmetry with respect to the internuclear axis and therefore can be written as

$$
\hat{\mathrm{h}}=-\frac{1}{2} \nabla^{2}+V(\xi, \eta)
$$

The function $V(\xi, \eta)$ is not specified at this point as it depends on the molecular system with one effective electron $\left(\mathrm{H}_{2}^{+}, \mathrm{Na}_{2}^{+}, \mathrm{HeH}^{+}\right.$etc.). $V(\xi, \eta)$ can represent external fields, core potentials, core polarizations etc. Due to the rotational symmetry, the orbitals $\psi$ obtained as solution of the OESE $(\hat{\mathrm{h}} \psi=\epsilon \psi)$ can be specified by quantum numbers $\lambda=0,1,2, \ldots$ and $m= \pm \lambda$, where $e^{\mathrm{i} m \phi}$ are the corresponding angular solutions. In case of homonuclear diatomic molecular systems like $\mathrm{H}_{2}^{+}$, there exists an additional inversion symmetry with respect to the center of internuclear axis $(V(\xi, \eta)=V(\xi,-\eta))$. This inversion symmetry leads to the following relations,

$$
\xi \rightarrow \xi, \quad \eta \rightarrow-\eta, \quad \phi \rightarrow \phi+\pi
$$

The eigenvalue $\mathcal{P}$ of the inversion operator is equal to 1 for gerade and -1 for ungerade states. Thus, $\{\lambda, m,[\mathcal{P}]\}$ (square bracket for optional quantum numbers) represent the set of quantum numbers for diatomic molecules.

The OESE is solved in a confined volume or an elliptical 'box'. The box is spanned by $\xi$ 


\section{Time-dependent Schrödinger equation}

and $\eta$ coordinates and knot-sequences are chosen in these two directions. The flexibility in choosing a knot sequence leads to an accurate description of wavefunction and energy. At each of these knot points the wavefunction is described by a B-spline of certain order. By using B-splines one gets a continuous description of the space within the box such that any wavefunction can be correctly described. The number and order of B-splines in each direction has to be chosen carefully and should be checked for convergence. The order and number of B-splines determine the quality of the basis set. Another important quantity is the box size. It should be sufficiently large and also checked for convergence. For further details of the solution of OESE see Reference [123].

The important parameters which enter the one electron input code are

The internuclear distance, $R$, at which the OESE is solved. The value of $R$ can be chosen from the open range $(R \in(0, \infty))$. The internuclear of zero shall correspond to $\mathrm{He}^{+}$(united atom limit). Since coordinates $(\xi$ and $\eta$ ) are scaled with $R$, the value of zero for internuclear distance will cause an error but a value infinitesimally close to zero will lead to united atom limit. The value of $R$ at infinity shall lead to the solution of a hydrogen atom and a hydrogen ion.

The range of quantum number $m$. The OESE solved for each symmetry and $m$ defines the number of symmetries that are to be calculated.

The properties of the potential within which the OESE is solved. The symmetry of the potential with respect to reflection of coordinates.

The maximum value of $\xi\left(\xi_{\max }\right)$. This is a dimensionless number. The box size can be extracted from $\xi_{\max }$ using

$$
\xi_{\max }=\frac{2 D_{\max }}{R}
$$

where $D_{\max }$ is the box size. The box size is important for controlling the density of states in the continuum. A larger box provides a higher density of states in the continuum. A large box is suitable for describing whole set of states (which also includes the continuum). In the case that only individual bound states are to be studied, a small box is sufficient. The box size should be sufficient to include the quiver radius of the electron in the field.

The $\operatorname{order}\left(O_{\xi}\right)$ and number $\left(N_{\xi}\right)$ of B-splines in $\xi$ direction. The number of B-splines controls the number of states that will be calculated for a particular symmetry (quantum number $m$ ). $N_{\xi}$ is dependent on the box size. There should be sufficient number of B-splines spanning the box in the $\xi$ direction. The B-splines can be spaced linearly or at specified positions. This is defined by a knot-sequence.

The order $\left(O_{\eta}\right)$ and number $\left(N_{\eta}\right)$ of B-splines in $\eta$ direction. For a symmetric potential like in the case of $\mathrm{H}_{2}^{+}$the $N_{\eta}$ is doubled. The total number of states that are produced for $\mathrm{H}_{2}^{+}$is $2 \times\left(N_{\xi}-1\right) \times N_{\eta}$. Here again one can choose a linear knotsequence or specify the position of B-Splines in $\eta$ direction. 
Potential $V(\xi, \eta)$ should be specified. In the case of an electron in the Coulomb field of two nuclei (eg. $\mathrm{H}_{2}^{+}, \mathrm{HeH}^{2+}$ ) it is sufficient to specify the charge on the two nuclei. In the case alkali dimer ions the model potential should be specified.

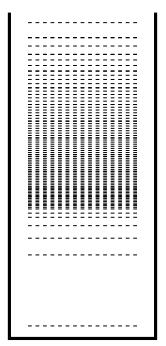

$(0, \mathrm{~g})$

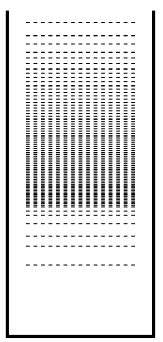

$(0, \mathrm{u})$

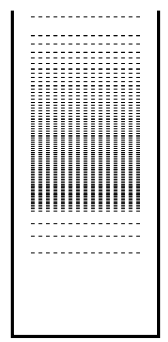

$(1, \mathrm{~g})$

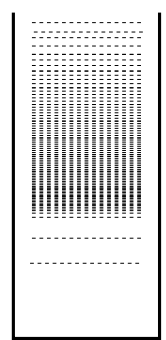

$(1, \mathrm{u})$
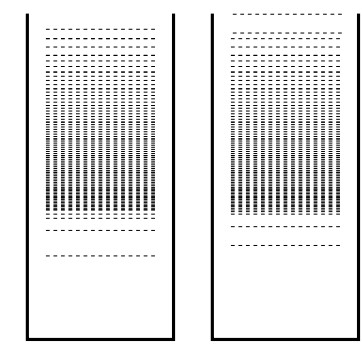

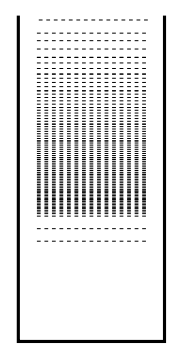

$(\mathrm{M}, \mathrm{g})$

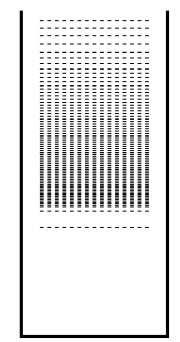

$(\mathrm{M}, \mathrm{u})$

Figure 2.2: A schematic representation of $\mathrm{H}_{2}^{+}$solution. Each block represents a symmetry adapted solution. $M$ is the maximum value of the quantum number $m$. The symbols "g" and "u" represent gerade and ungerade symmetry respectively.

Figure 2.2 shows a schematic representation of $\mathrm{H}_{2}^{+}$solution. The OESE solution for $\mathrm{H}_{2}^{+}$is obtained for each symmetry represented by the quantum number $m$ and gerade(g) or ungerade(u) symmetry. The quantum number $m$ has values $0,1, \cdots$ and goes up to the chosen maximum value, $M$. The solution for each symmetry contains a finite number of states depending on the choice of B-splines. These states span a wide range of energy values, starting from the ground state of a particular symmetry to the very energetically high continuum states. The very high lying states may not be the physical states of $\mathrm{H}_{2}^{+}$but may come from the solution of particle in box. Each state in the figure is represented by a dashed line. Since the Rydberg states and continuum states are very close to each other, they are seen as a block.

If a time-dependent solution of $\mathrm{H}_{2}^{+}$in a laser field is needed, the symmetry allowed electronic transition dipole moments are calculated and a time-propagation is performed. The time-propagation scheme for $\mathrm{H}_{2}^{+}$and $\mathrm{H}_{2}$ is the same and will be discussed in Section 2.3.

\subsection{Two-electron Schrödinger equation}

The two-electron Schrödinger equation (TESE),

$$
\hat{\mathrm{H}}(1,2) \Psi(1,2)=\left(\hat{\mathrm{h}}(1)+\hat{\mathrm{h}}(2)+\frac{1}{r_{12}}+V_{A B}(R)\right) \Psi(1,2)=E \Psi(1,2),
$$

is solved using the configuration interaction (CI) approach. The potential $V_{A B}(R)$ is either the Coulomb potential or the interaction potential between the two cores. The total set of quantum numbers is represented by $\Gamma$, consists of $\Gamma \equiv\left\{\Lambda, M_{D}, S,\left[P, P^{\Sigma}\right]\right\}$. 


\section{Time-dependent Schrödinger equation}

$\Lambda$ is the absolute value of the component of the total angular momentum along the internuclear axis $(\Lambda=0(\Sigma), 1(\Pi), 2(\Delta), \ldots)$ and $M_{D}= \pm \Lambda . S$ is the total spin $(S=0,1)$. The optional quantum numbers $P$ and $P^{\Sigma}$ specify the parity with respect to inversion symmetry (gerade or ungerade ) and, for $\Sigma$ states only, with respect to a reflection at a plane through the nuclei. For further technical details see [123].

Normalized and fully symmetry-adapted configurations $\Upsilon_{i}^{\Gamma}$ are used in the CI calculations. The configurations are build with the aid of products of two orbitals

$$
|\nu \gamma \bar{\nu} \bar{\gamma}\rangle \equiv \psi_{\nu}^{\gamma}\left(\xi_{1}, \eta_{1}, \phi_{1}\right) \psi_{\bar{\nu}}^{\bar{\gamma}}\left(\xi_{2}, \eta_{2}, \phi_{2}\right),
$$

with $\bar{\gamma} \equiv\{\bar{\lambda}, \bar{m}[\mathcal{P}]\}$. For a given configuration $\Upsilon_{i}^{\Gamma} \equiv[\nu \gamma \bar{\nu} \bar{\gamma}]$ the orbitals $\nu \gamma$ and $\bar{\nu} \bar{\gamma}$ satisfy the conditions $m+\bar{m}=M$ and $\mathcal{P} \overline{\mathcal{P}}=\mathrm{P}$. For non- $\Sigma$ states $(\Lambda \neq 0)$ particle-exchange symmetry is considered using

$$
[\nu \gamma \bar{\nu} \bar{\gamma}]_{\Lambda \neq 0}=\frac{|\nu \gamma \bar{\nu} \bar{\gamma}\rangle+(-1)^{S}|\bar{\nu} \bar{\gamma} \nu \gamma\rangle}{\sqrt{2\left(1+\delta_{\nu \bar{\nu}} \delta_{\gamma \bar{\gamma}}\right)}} .
$$

For $\Sigma$ states $(\Lambda=0)$ inclusion of the additional reflection symmetry leads to

$$
[\nu \gamma \bar{\nu} \bar{\gamma}]_{\Lambda=0}=\frac{\left(|\nu \gamma \bar{\nu} \bar{\gamma}\rangle+(-1)^{S}|\bar{\nu} \bar{\gamma} \nu \gamma\rangle\right)+\mathcal{P}^{\Sigma}\left(|\nu \gamma \bar{\nu} \bar{\gamma}\rangle+(-1)^{S}|\bar{\nu} \bar{\gamma} \nu \gamma\rangle\right)^{*}}{\sqrt{2\left(1+\delta_{\nu \bar{\nu}} \delta_{\gamma \bar{\gamma}}\right)\left(1+\delta_{0 m} \delta_{0 \bar{m}}\right)}},
$$

where it was used that two different orbitals with the same $\nu$ and $|\gamma|$ can be obtained from each other by complex conjugation. Using the relation

$$
|\nu \gamma \bar{\nu} \bar{\gamma}\rangle^{*} \equiv\left|\nu \gamma^{*} \bar{\nu} \bar{\gamma}^{*}\right\rangle
$$

configuration (Equation 2.8) can finally be represented as a linear combination of products of two orbitals. Clearly, the choice of $\nu \gamma$ and $\bar{\nu} \bar{\gamma}$ has always to be done in such a way that a double occurrence of the same configuration is prevented. The two-electron problem

$$
\hat{\mathrm{H}}(1,2) \Psi_{\mu}^{\Gamma}(1,2)=E_{\mu}^{\Gamma} \Psi_{\mu}^{\Gamma}(1,2)
$$

is solved by an expansion of the wavefunctions as linear combinations of $N_{\Gamma}$ configurations,

$$
\Psi_{\mu}^{\Gamma}(1,2)=\sum_{i=1}^{N_{\Gamma}} C_{\mu i}^{\Gamma} \Upsilon_{i}^{\Gamma}(1,2)
$$

The coefficients $\left\{C_{\mu i}^{\Gamma}\right\}$ and energies $E_{\mu}^{\Gamma}$ are calculated using LAPACK subroutine DSPEVX. For further technical details see [123].

The results should be checked for convergence by including additional configurations from higher or lower angular momentum states. After successfully calculating the energies and wavefunctions for the two-electron molecule, the symmetry allowed electronic transition dipole moments are also calculated. 


\subsection{Time propagation}

A general approach for solving the time-dependent Schrödinger equation (TDSE) for molecules was developed in this work. Such an approach has already been successfully applied to one- and two-electron atoms exposed to laser fields (see [115] for a review), and recently for $\mathrm{H}_{2}^{+}$molecule [116]. The method can be applied to systems where the timedependent interaction takes place over a finite time. The time-dependent Schrödinger equation (TDSE) is solved by expanding the time-dependent wave function in terms of field-free (time-independent) states. The total in-field Hamiltonian is given by

$$
\hat{\mathrm{H}}(t)=\hat{\mathrm{H}}_{0}+\hat{\mathrm{D}}(t),
$$

where $\hat{\mathrm{H}}_{0}$ is the field-free electronic Born-Oppenheimer Hamiltonian of a molecule and $\hat{\mathrm{D}}(t)$ is the operator describing its interaction with the (time-dependent) laser field. The non-relativistic approximation is used for both operators, and the interaction is described within the dipole approximation. In velocity gauge, $\hat{\mathrm{D}}(t)=\mathbf{A}(t) \cdot \mathbf{p} / c$, and in length gauge, $\hat{\mathrm{D}}(t)=-\mathbf{F}(t) \cdot \mathbf{r}$. (Here, and in the following, atomic units $\left(e=m_{e}=\hbar=1\right)$ are used unless specified otherwise.) $\mathbf{A}$ is the vector potential of the laser field, $\mathbf{p}$ is the total momentum operator of the electrons, $c$ is the velocity of light in vacuum, $\mathbf{F}(t)$ is the electric field vector and $\mathbf{r} \equiv \mathbf{r}_{1}, \mathbf{r}_{2}$ is the position vector of the electrons. The resulting TDSE

$$
\mathrm{i} \frac{\partial}{\partial t} \Psi(\mathbf{r}, t)=\hat{\mathrm{H}} \Psi(\mathbf{r}, t)
$$

is solved by expanding the wave function $\Psi$ according to

$$
\Psi(\mathbf{r}, t)=\sum_{n L} b_{n L}(t) \phi_{n L}(\mathbf{r})
$$

in terms of the time-independent wave functions $\phi_{n L}$. The latter are solutions of the field-free molecular Schrödinger equation

$$
\hat{\mathrm{H}}_{0} \phi_{n L}(\mathbf{r})=E_{n L} \phi_{n L}(\mathbf{r}) .
$$

The compound index $L$ represents the total angular momentum $(\Sigma, \Pi, \ldots)$ and the symmetry gerade or ungerade, and in the case of $\Sigma$ symmetry $P^{\Sigma}= \pm 1 . \quad n$ is just an index of a state with the particular symmetry $L$. The solution of the time-independent Schrödinger equation is in this approach obtained by solving Equation 2.15 within a finite volume or a box. The solution within a finite volume leads to discretized states. Thus, the index $n$ remains discrete even for states in the electronic continuum. The solution of Equation 2.15 for $\mathrm{H}_{2}$ can be obtained by doing a configuration interaction (CI) using $\mathrm{H}_{2}^{+}$ orbitals or using density-functional theory (DFT) within single-active-electron (SAE) approximation. The details of obtaining the solution of time-independent Schrödinger equation will be discussed in the next chapter. What is needed for the solution of the TDSE is the discretized, symmetry adapted electronic solution of the time-independent Schrödinger equation. 


\section{Time-dependent Schrödinger equation}

Substitution of Equation (2.14) into the TDSE [Equation (2.13)], multiplication of the result by $\phi_{m K}^{*}$, and integration over the electronic coordinates yields

$$
\mathrm{i} \frac{\partial}{\partial t} b_{m K}(t)=E_{m K} b_{m K}(t)+\sum_{n L} D_{m K, n L}(t) b_{n L}(t),
$$

with $D_{m K, n L}(t)=\left\langle\phi_{m K}|\hat{\mathrm{D}}(t)| \phi_{n L}\right\rangle$. It should be emphasized that with this approach the complete time dependence is incorporated in the coefficients $b_{n L}$. The coefficients $b_{n L}$ are complex and can be broken into real and imaginary parts for further simplification,

$$
b_{n L}=b_{n L}^{\mathrm{r}}+\mathrm{i} b_{n L}^{\mathrm{i} m} .
$$

This breakup into real and imaginary parts leads to

$$
\begin{gathered}
-\frac{\partial}{\partial t} b_{m K}^{\mathrm{im}}(t)=E_{m K} b_{m K}^{\mathrm{r}}(t)+\sum_{n L} D_{m K, n L}(t) b_{n L}^{\mathrm{r}}(t), \\
\frac{\partial}{\partial t} b_{m K}^{\mathrm{r}}(t)=E_{m K} b_{m K}^{\mathrm{i} m}(t)+\sum_{n L} D_{m K, n L}(t) b_{n L}^{\mathrm{i} m}(t) .
\end{gathered}
$$

The Equations 2.18 and 2.19 in real variables and can be solved numerically by using a solver for the coupled first-order differential equations. There are many free and commercial solvers available for the problem. Here, a commercial NAG routine, D02CJF, based on variable-order, variable-step Adams solver was chosen for its efficiency and time saving abilities. A fourth order Runge-Kutta solver was also implemented. There are other NAG routines like D02BJF and D02EJF which can also solve the same first-order ordinary differential equation.

The laser-pulse parameters are contained in $D_{m K, n L}(t)$. A laser pulse can be characterized by its intensity, central frequency and spatial time profile. The present day ultrashort high intensity laser pulses are difficult to characterize. The intensity estimation is not accurate and an error of $10 \%$ is common. The monochromaticity of such short laser pulses is another issue. Techniques like spectral phase interferometry for direct electric-field reconstruction (SPIDER) can be used to get the spatial profile. For theoretical purposes all these errors can be neglected and one can use a perfect laser pulse with an exact intensity and wavelength. For the spatial time profile different choices for the temporal shape of the pulse are implemented. Depending on the choice of gauge, one can either use the electric field $(\mathbf{F}(t))$ or the vector potential $\mathbf{A}(t)$ for the laser field description. The peak laser intensity $I$ is given by

$$
I=\frac{1}{8 \pi} c F_{0}^{2} .
$$

Here, $c$ is the velocity of light in vacuum, $c=137.035999$, which is the inverse of the fine structure constant. $F_{0}$ is the peak/maximum value of the electric field. The electric 
field and the vector potential are related by the following relation

$$
\mathbf{F}(t)=-\frac{\partial}{\partial t} \mathbf{A}(t)
$$

Let $C(t)$ denote the amplitude of the electric field $\mathbf{F}(t)$ or the vector potential $\mathbf{A}(t)$. In order to model the pulse following relations can be chosen in the code :

$$
\begin{gathered}
C(t)=C_{0} \cos ^{2}\left(\frac{\pi t}{\tau}\right) \cos \left(\omega t+\phi_{\mathrm{C} E P}\right), \\
C(t)=C_{0} \exp \left(-\frac{2 t^{2}}{\tau_{g}^{2}}\right) \cos \left(\omega t+\phi_{\mathrm{C} E P}\right), \\
C(t)=C_{0} \operatorname{sech}\left(\frac{t}{\tau_{h}}\right) \cos \left(\omega t+\phi_{\mathrm{CEP} P}\right) .
\end{gathered}
$$

Here, $C_{0}$ represents the maximum value of the quantity chosen $(A(t)$ or $F(t))$ for pulse modeling. If one chooses the vector potential, the electric field can be calculated by using Equation 2.21 and vice-versa. $\omega$ is the photon energy or the frequency, $t$ is the time and $\phi_{\mathrm{CEP}}$ is the carrier-envelope phase. $\tau$ is the total pulse time, and $\tau_{g}$ and $\tau_{h}$ are characteristic times for Gaussian and hyperbolic secant pulses respectively.

The most significant part of the interaction between the laser and the molecule takes place when the laser intensity is maximum. In order to have these pulses behave in a similar way, the full width at half maximum (FWHM) of all these pulses should be kept constant. Once the FWHM is the same, then one can investigate the effect of pulse shape and other parameters. The FWHM of a cosine squared envelope pulse $\left(\Gamma_{c}\right)$ with duration $\tau$ is $\tau / 2$. The FWHM for a Gaussian envelope pulse $\left(\Gamma_{g}\right)$ as described in Equation 2.23 is given by

$$
\Gamma_{g}=\sqrt{\left(-2 \ln \left(\frac{1}{2}\right)\right)} \tau_{g}
$$

The FWHM for hyperbolic secant pulse $\left(\Gamma_{h}\right)$ as described in Equation 2.24 is

$$
\Gamma_{h}=2.63392 \tau_{h} .
$$

Thus, $\Gamma_{c}=\Gamma_{g}=\Gamma_{h}$ is the condition that different pulses behave in a similar manner.

The amplitude of the pulse with a cosine squared envelope goes to zero at the end of the pulse. Thus the time for the propagation of the numerical routine is easily fixed. In case of pulses with Gaussian or hyperbolic secant envelopes, it is difficult to define a propagation time because the amplitude goes to zero only at infinity. The numerical time propagation routine can not propagate for infinite time. Thus, we need to cut the wings of the pulse extending to infinity. A propagation time of $4 \Gamma_{c}$ and $6 \Gamma_{h}$ is chosen for Gaussian and hyperbolic secant pulses respectively. Figure 2.3 shows the different pulses implemented in the code. The laser wavelength is $800 \mathrm{~nm}$ laser and FWHM of 3 cycles is used. The amplitude of electric field is fixed to unity. As seen from the figure, 


\section{Time-dependent Schrödinger equation}

different pulses are close to each other in the center region and start to deviate as the time increases. It is also clear from the figure that Gaussian and hyperbolic secant pulses need more time for numerical propagation for the same FWHM.

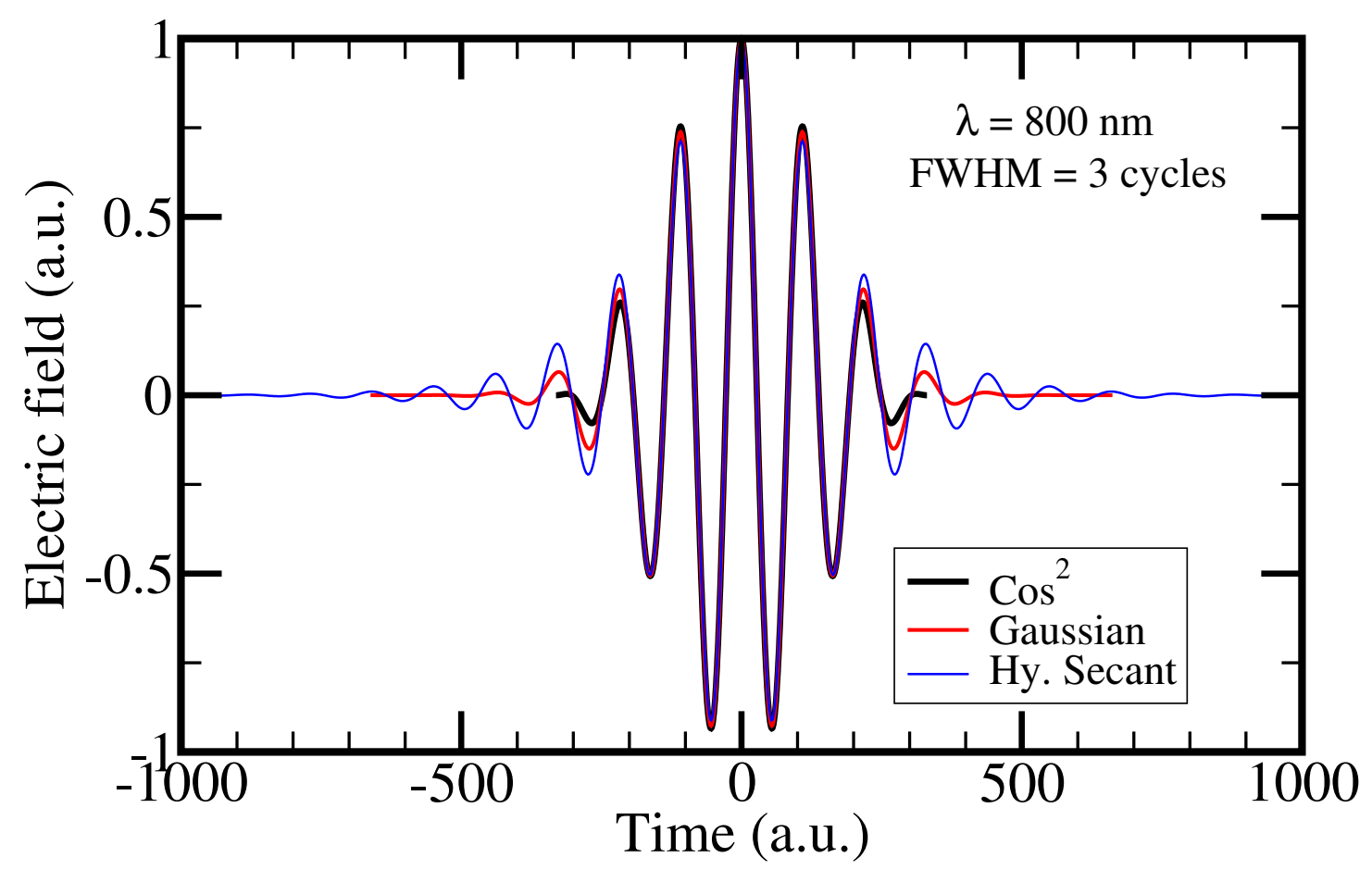

Figure 2.3: Electric field as a function of time for different pulses is shown. The peak value of the electric field for all the pulses is fixed to unity. A $800 \mathrm{~nm}$ laser with FWHM of 3 cycles is used. The cosine squared pulse is shown by black line, Gaussian pulse is shown by red line and hyperbolic secant pulse is shown by blue line.

The carrier envelope phase $\phi_{\mathrm{CEP}}$ describes the absolute phase of the pulse. It can be chosen to have any arbitrary value between $[0,2 \pi]$. The peak intensity should be maximum at the center of the pulse. In order to achieve this, $\phi_{\mathrm{CEP}}=0$ is chosen for the $\mathbf{F}(t)$ based pulses and $\phi_{\mathrm{CEP}}=\frac{\pi}{2}$ for $\mathbf{A}(t)$ based pulses. 


\section{SAE-TDSE}

The theory of strong field physics relies heavily on single active electron (SAE) approximation. Most of the theories or models which were initially developed for hydrogen atom were further extended to multi-electron atoms and molecules using SAE approximation. Most common example is the theory of tunnel ionization. The theory of tunnel ionization was developed for hydrogen like atoms (atoms with single electron or effectively single electron) and is commonly known as ADK model [102]. The ADK model was extended to molecules by Lin and co-workers [103] and is commonly called MO-ADK (molecular ADK). The ADK and MO-ADK models are discussed in more detail in Chapter 4. The strong field approximation (SFA) or Intense-field many-body $S$-matrix theory (see [107] for a review) is another SAE theory which has been very successful.

Within the SAE approximation ionization is described as a pure one-electron process in which all remaining (non-ejected) electrons act as frozen spectators. An intrinsic ambiguity of SAE is introduced by the description of the potential created by that part of the atomic or molecular system that does not respond to both the laser field and the change of the potential due to the active electron that responds to the laser field. Since electrons are indistinguishable, the need for an artificial distinction between the active electron and the inactive ones necessarily leads to formal inconsistencies as was discussed, e.g., in [124]. Besides model potentials mean-field theories that yield orbitals allow to implement SAE in the most straightforward way. Therefore, mostly Hartree-Fock (HF) was used in the implementation of SAE in the context of MO-ADK or MO-SFA (molecular SFA). Freezing all but one electron leads then to a frozen-core HF (static-exchange) description.

The applicability of the SAE in which the relaxation of the remaining electrons as well as the action of the laser field on them is completely ignored is so far unclear. The success of the TDSE applied to hydrogen atom (being pioneered in [125]) led to development of SAE-TDSE as demonstrated in [126-128] seems to indicate that very often intense laser fields primarily act on isolated electrons. This is further substantiated by the success of the SAE-based SFA for a large number of atoms and laser parameters reported, e. g., in [107]. Clearly, SAE fails, if doubly excited states are important. For systems with delocalized $\pi$ electrons non-adiabatic multi-electron behavior was discussed in [129]. It was shown in [130] that suppressed ionization in fullerenes can only be described by many-electron tunneling model. However, it was thereafter demonstrated that at least the experimental results for fullerenes in [131] can alternatively also be explained with the aid of MO-SFA-VG (molecular SFA in velocity gauge) and thus within SAE [132].

In the present work an approach for solving the TDSE within SAE approximation for molecules exposed to laser fields is developed. The SAE-TDSE approach is similar to the one used for hydrogen molecule as shown in Chapter 2. This work is a result of a 
collaboration between three groups. The solution of TISE within SAE approximation for molecules was provided by Piero Decleva (University of Trieste) and Alberto Castro (Free University, Berlin) and time-propagation was performed by our group. The main task of the present work was to perform the time-propagation and look at convergence with respect to laser parameters and various basis set parameters used in TISE.

In the present SAE-TDSE implementation a different approach was chosen by describing the inactive electrons with the aid of density-functional theory (DFT). From now this method will be referred as DFT-SAE-TDSE. The advantage of DFT-SAE-TDSE compared to $\mathrm{HF}$ is the ability to include in an approximate way correlation in the description of the inactive electrons. The price to pay is the unknown exact functional.

Clearly, the validity of the SAE that in fact should depend on the molecular system and the laser-pulse parameters can only be judged on the basis of very accurate experimental data or full many-electron calculations. An advantage of the latter is that not only all possible ambiguities with respect to the spatio-temporal shape of the laser pulse can be removed, but it is also possible to separate purely electronic effects from the ones arising from orientation or vibrational motion. Therefore, molecular hydrogen for which very accurate two-electron calculations can be performed using grid based method [133] or the spectral method (CI-TDSE, a part of the present work and shown in Chapter 2) $[134,135]$ was chosen as a first test case for the newly developed DFT-SAETDSE code for molecules. In this work, the results obtained for molecular hydrogen are directly compared for the different functionals that can be used within the DFT framework. It should be stressed, however, that DFT-SAE-TDSE is a general approach that can be applied even to large polyatomic systems. The results for $\mathrm{H}_{2}$ are shown in Chapter 7 and some larger molecular systems are shown in Chapter 9.

\subsection{Method}

The non-relativistic time-dependent Hamiltonian describing the electrons of a molecular system exposed to a laser pulse where the latter is treated classically can be written as

$$
\hat{\mathrm{H}}(t)=\hat{\mathrm{H}}_{\mathrm{mol}}+\hat{\mathrm{H}}_{\mathrm{int}}(t) .
$$

$\hat{\mathrm{H}}_{\text {mol }}$ is the time-independent Hamiltonian describing the electronic motion of the molecular system while $\hat{\mathrm{H}}_{\mathrm{i} n t}(t)$ represents the time-dependent laser-electron interaction. The Hamiltonian is the same as described in Equation 2.12 in Chapter 2, a different notation is chosen to distinguish between CI and SAE approaches. In the dipole approximation that is adopted here the interaction term may be expressed in the length or velocity gauge form. The wavefunctions obtained with either gauge differ only by a phase factor. Therefore, all physical observables obtained with the two wavefunctions agree, if the wavefunctions are exact. In practice, the use of a finite representation for the wavefunction may lead to differences, if the results are not sufficiently converged. In turn, the convergence behavior is gauge dependent, since the finite basis has to represent the phase factor occurring in one gauge but absent in the other one. As already shown in 
Section 2.3 the interaction term is then given by

$$
\hat{\mathrm{H}}_{\mathrm{i} n t}(t)=\frac{\mathbf{A}(t) \cdot \mathbf{P}}{c}=-\mathbf{F}(t) \cdot \mathbf{r}
$$

where $c$ is the vacuum speed of light, $\mathbf{A}(t)$ the time-dependent vector potential describing the laser pulse, and $\mathbf{P} \equiv \sum_{i} \mathbf{p}_{i}$ the momentum operator which is the sum over the momentum operators of the individual electrons. $\mathbf{F}(t)$ is the electric field vector and $\mathbf{r} \equiv \mathbf{r}_{1}, \mathbf{r}_{2}, \mathbf{r}_{3}, \cdots$ is the position vector of the electrons.

In the SAE it is assumed that only a single electron responds to the laser field while all other electrons remain unaffected. In the usual interpretation of the SAE in the context of atoms or molecules exposed to strong fields this implies also that no relaxation due to a possible excitation or even ionization of the active electron is allowed for. Expressing the many-electron wavefunction in form of a single Slater determinant built by orthonormal one-electron wavefunctions $\phi_{j}$ that are eigenfunctions of $\hat{\mathrm{H}}_{\text {mol }}$, freezing all but one electron, and using orthonormality as well as Slater-Condon rules leads finally to an effective one-electron Hamiltonian,

$$
\hat{\mathrm{h}}(t)=\hat{\mathrm{h}}_{\mathrm{mol}}+\hat{\mathrm{d}}_{\mathrm{int}}(t) .
$$

The dipole interaction term $\hat{\mathrm{d}}_{\mathrm{int}}(t)$ is equal to $\hat{\mathrm{H}}_{\mathrm{i} n t}(t)$ in Equation (3.2) but with the single-electron momentum operator $\hat{\mathrm{p}}$ instead of the total momentum operator $\hat{\mathrm{P}}$. The operator $\hat{\mathrm{h}}_{\text {mol }}$ describes the motion of the active electron in the potential formed by the nuclei and the remaining frozen electrons. For the single-determinant approximation this leads to to the frozen Hartree-Fock, or static-exchange Hamiltonian $\hat{\mathrm{h}}_{\mathrm{mol}}$. However, additional correlation effects can be formally included employing an appropriate optical potential. A simplified version is the use of a Kohn-Sham (KS) Hamiltonian

$$
\hat{\mathrm{h}}_{\mathrm{K} S}(\mathbf{r})=-\frac{1}{2} \nabla_{r}^{2}-\sum_{j=1}^{N} \frac{Z_{j}}{\left|\mathbf{r}-\mathbf{R}_{j}\right|}+\int \frac{n\left(\mathbf{r}^{\prime}\right)}{\left|\mathbf{r}-\mathbf{r}^{\prime}\right|} \mathrm{d} \mathbf{r}^{\prime}+V_{\mathrm{xc}}[n(\mathbf{r})]
$$

where $N$ is the number of nuclei whose position vectors are $\mathbf{R}_{j}, n(\mathbf{r})$ is the ground-state electron density, and $V_{\mathrm{xc}}$ the exchange-correlation potential. (Note that the parametrical dependence of $n$ and other quantities like the KS orbitals on the nuclear geometry is omitted here and in the following for reasons of better readability.)

The advantage of the KS Hamiltonian is that the numerically expensive calculation of the exchange integrals is avoided. This allows in principle to treat large molecular systems and saves computational resources. A further important advantage compared to the HF Hamiltonian is the ability to include correlation into the calculation of the core. An evident disadvantage is the unknown exact functional. As is discussed below, the proper choice of a functional is important for the success of the present approach. Note, there is no principle obstacle to use HF or a more elaborate many-body potential, at a cost of a more involved computational treatment. In fact, for the example of $\mathrm{H}_{2}$ considered in this work, HF-based SAE calculations are performed for comparison. For this purpose, the static potential generated by a singly occupied Hartree-Fock orbital 
$\phi_{1 \sigma_{g}}^{\mathrm{HF}}$ was employed for the calculation of the excited states. In this case $\hat{\mathrm{h}}_{\mathrm{K} S}$ in Equation (3.4) is substituted by the Hartree-Fock Hamiltonian $\hat{\mathrm{h}}_{\mathrm{H} F}$, if $n(\mathbf{r})=\left|\phi_{1 \sigma_{g}}^{\mathrm{H} F}(\mathbf{r})\right|^{2}$ and $V_{\mathrm{xc}}=0$ are used.

The evaluation of the KS orbitals that solve the eigenvalue problem

$$
\hat{\mathrm{h}}_{\mathrm{K} S}(\mathbf{r}) \phi_{i}(\mathbf{r})=\epsilon_{i} \phi_{i}(\mathbf{r})
$$

is performed in the two-step procedure described in detail in [136]. First, a conventional bound-state DFT calculation using the linear combination of atomic orbitals (LCAO) approach is performed using program ADF [137, 138]. This program adopts Slater-type orbitals as basis functions. The obtained electron density $n(\mathbf{r})$ is then used to set up the matrix of the KS Hamiltonian (3.4) in an alternative set of basis functions that is more suitable for the present purpose.

These basis functions consist of a product of a symmetry-adapted linear combination of real spherical harmonics $\left(Y_{l m}^{R}(\theta, \phi)\right)$ and radial B-spline functions,

$$
\chi_{n, l, h, \lambda, \mu}^{s}(\mathbf{r})=\sum_{j \in Q_{s}} \frac{B_{n}^{k}\left(r_{j}\right)}{r_{j}} \sum_{m=-l}^{l} b_{l, m, h, \lambda, \mu, j} Y_{l m}^{R}\left(\theta_{j}, \varphi_{j}\right) .
$$

In Equation (3.6) $s$ is an index that is related to the center of the basis function which defines the origin of a local spherical coordinate system for $\mathbf{r}_{j}=\left\{r_{j}, \theta_{j}, \varphi_{j}\right\}$. The origin of the central coordinate system is denoted by $s=0$. It is usually chosen to agree with the center of charge of the molecule. In the spirit of the LCAO approach $s>0$ runs over the non-symmetry equivalent nuclei and defines therefore atom-centered basis functions. The molecular symmetry is accounted for by the sum over $j$. It runs over the number $Q_{s}$ of nuclei that are symmetry-equivalent to nucleus $s$. The coefficients $b_{l, m, h, \lambda, \mu, j}$ are also determined by the molecular symmetry and provide symmetry-adapted linear combinations of the spherical harmonics. The indices $\lambda, \mu$, and $h$ specify the irreducible representation (IR), the subspecies in case of degenerate IRs, and an identifier in case of different elements with agreeing $\{l, \lambda, \mu\}$, respectively. Finally, $B_{n}^{k}$ specifies the $n$th B spline of order $k$.

The radial B-spline functions are defined by a grid of knots and the interval $\left[0, r_{\max }^{s}\right]$ covered by the knot sequence. In the present implementation of the code the values of $r_{\max }^{s}$ for $s>0$ have to be chosen in such a way that the spheres defined by different values of $s>0$ do not overlap. Continuity of the second derivative over the surfaces of these spheres is achieved by removing the last three B-spline functions of each set. The set of central basis functions denoted by $s=0$ covers on the other hand a large sphere (with radius $r_{\max }^{0}$ ) that includes all other spheres. In fact, for the present application, $r_{\max }^{0}$ is chosen much larger, since its value determines the number of Rydberg states and the density of states in the electronic continuum that are obtained with a given basis set. The reason is that for a given value of $r_{\max }^{0}$ the diagonalization of the KS Hamiltonian in the given basis yields only those states whose density is confined to a smaller volume or that (accidentally) happen to possess a node at $r_{\max }^{0}$. Although it is possible to obtain alternatively any continuum state using the so-called free-boundary approach $[136,139]$, 
the "box" discretization achieved by a fixed-boundary approach appears more suitable for the present purpose. The reason is that the discretized continuum obtained this way fulfills a number of sum rules and allows therefore to substitute integrals over continuum states to be replaced by simple sums over their discretized counterparts.

Once the set of eigenfunctions $\phi$ of the field-free KS Hamiltonian (3.4) has been obtained, one of the orbitals that is occupied in the initial state can be selected as the active one. It is used together with all initially unoccupied orbitals as a basis for solving the time-dependent Schrödinger equation defined by the Hamiltonian in Equation (3.3). Now the time-propagation is performed in the same way as shown in Section 2.3. Insertion of

$$
\Psi(\mathbf{r}, t)=\sum_{i, \lambda, \mu} c_{i, \lambda, \mu}(t) \phi_{i, \lambda, \mu}(\mathbf{r})
$$

into

$$
i \frac{\partial \Psi(\mathbf{r}, t)}{\partial t}=\hat{\mathrm{h}}(t) \Psi(\mathbf{r}, t)
$$

and multiplication from left with $\phi_{j, \kappa, \nu}^{*}(\mathbf{r})$ yields after integration over the electronic coordinates

$$
i \frac{\mathrm{d} c_{j, \kappa, \nu}(t)}{\mathrm{d} t}=\epsilon_{j, \kappa, \nu} c_{j, \kappa, \nu}(t)+\sum_{i, \lambda, \mu} d_{j, \kappa, \nu ; i, \lambda, \mu} c_{i, \lambda, \mu}(t) .
$$

In Equation (3.9) the electronic dipole transition matrix elements $d_{j, \kappa, \nu ; i, \lambda, \mu}=\left\langle\phi_{j, \kappa, \nu}|\hat{\mathrm{d}}(t)| \phi_{i, \lambda, \mu}\right\rangle$ were introduced. Depending on the molecular symmetry different dipole-selection rules apply. This leads to various block structures of the coupled set of ordinary first-order differential equations (3.9). The time-dependent coefficients $c_{i, \lambda, \mu}$ are obtained numerically using an variable-order, variable-step Adams routine. The approach for solving the TDSE is thus very similar to the one implemented earlier for treating the full electronic problem of $\mathrm{H}_{2}$ exposed to intense laser pulses described in [134] that is also used in the present work in order to obtain reference data. The only difference is that in the DFT-SAE-TDSE approach an effective one-electron Hamiltonian is used together with KS orbitals while previously, as shown in Chapter 2, the full two-electron Hamiltonian is adopted using configuration interaction (CI) wavefunctions. In the resulting CI-TDSE approach Equation (3.1) is solved (instead of Equation (3.3)) by an expansion of the time-dependent two-electron wavefunction in terms of field-free CI wavefunctions describing $\mathrm{H}_{2}$ in the fixed-nuclei approximation. This gives essentially exact results for the non-relativistic electronic problem of $\mathrm{H}_{2}$ exposed to a laser pulse within the dipole approximation.

\subsection{Computational details}

Besides the limitations intrinsic to the SAE the accuracy of the present numerical approach is influenced by the chosen potential $V_{\mathrm{x} c}$. While the basis-set dependence can be systematically investigated by a variation of the basis-set parameters, the choice of $V_{\mathrm{xc}}$ 
incorporates an unknown systematic error. In the present work the influence of both the basis set as well as the one of $V_{\mathrm{xc}}$ was investigated.

The question of basis-set quality may be further split by considering two different issues. One is the description of the molecular ground-state properties, the other is its ability to properly describe the time-dependent wavefunction of the active electron in the combined potential of the remaining electrons and the laser field. The description of the molecular ground-state properties and thus the quality of the KS Hamiltonian (3.4) is determined by the STO basis used in the ADF program run and the completeness especially of the nuclei-centered B-spline basis functions $\left(\chi_{n, l, h, \lambda, \mu}^{s}\right.$ with $\left.s \neq 0\right)$. In the present work both DZP (two 1s and one 2p STO functions) and extended, even-tempered ET (four 1s, two 2p, and one 3d STO functions) basis sets were tested. All results agreed within the graphical resolution of the figures shown in this work.

The main basis-set parameters responsible for an adequate description of the active electron itself are the radius $r_{\max }^{0}$ of the central sphere in which the radial basis is confined, the number of B splines, and the number of angular momenta $l$ specified by $l_{\max }$. Since a linear grid sequence was used for the knot points, the number of B splines fixes together with $r_{\max }^{0}$ the spacing $\Delta r$ between the knots. A further parameter is the order $k$ of the $\mathrm{B}$ splines. However, an increase of the order allows only to achieve a similar result with a sparser knot sequence. Therefore, the investigation of convergence with respect to either number of B splines or their order is somehow redundant in the sense that if convergence is achieved with respect to one parameter, it should also be obtained with respect to the other. It is often practical to introduce an energy cut-off $\epsilon_{\text {cut }}$ as a further parameter. In this case only those KS orbitals are included in the expansion (3.7) of the time-dependent wavefunction $\Psi$ that possess an energy smaller than $\epsilon_{\mathrm{cut}}$.

It was found that converged excitation and ionization yields were obtained for the investigated ranges of laser parameters when choosing $r_{\max }^{0} \geq 120 a_{0}$ with a linear spacing of the knot sequence $\Delta r \leq 0.65 a_{0}$. This was confirmed by performing calculations varying $r_{\max }^{0}$ in between 120 and $240 a_{0}$ and by a variation of $\Delta r$ between 0.70 and $0.40 a_{0}$. A variation of the highest angular momentum $l_{\max }$ between 10 and 16 revealed no visible differences. Convergence with respect to $\epsilon_{\text {cut }}$ was investigated by a variation of this parameter in steps of $10 \mathrm{a}$. u. in between 10 and $40 \mathrm{a}$. u. Good convergence was found. All shown results were obtained with $\epsilon_{\text {cut }}=40 \mathrm{a}$. u.

In addition to the simple Hartree (static) potential generated by the Hartree-Fock $1 \sigma_{g}$ orbital of $\mathrm{H}_{2}$, a number of DFT $V_{\mathrm{x} c}$ potentials were tested in order to investigate the sensitivity of the results on the chosen functional. The ground-state local-density approximation LDA [140] using the VWN functional [141], the transition-state LDA (TSLDA) with half electron removed that gives much better transition energies, and the LB94 functional [142] have been employed. TSLDA consists in the application of Slater's transition-state technique to the LDA functional [143]. LB94 ensures the correct Coulombic asymptotic behavior and gives better results for polarizabilities as well as single photon excitation and photoionization spectra [144].

Molecular hydrogen was chosen as a first test case of the DFT-SAE-TDSE approach, since it is possible to compare with full two-electron calculations. As described in detail in 
Chapter 2 the two-electron code solves the TDSE in a basis of field-free states. The latter are, however, now fully correlated solutions of the complete two-electron Hamiltonian. The field-free wavefunctions are obtained from a CI calculation performed in the basis of orbitals that are solutions of the one-electron Hamiltonian in which the electron-electron interaction is completely neglected. The orbitals are expressed in prolate spheroidal coordinates $(1 \leq \xi<\infty,-1 \leq \eta \leq 1,0 \leq \varphi<2 \pi)$ and expanded in products of B splines along the two radial coordinates, while the angular part is simply given by the cylindrical symmetry and thus by the exponential functions $\exp (\operatorname{im} \varphi)$. Details concerning the used CI method can be found in Section 2.2 or [123]. The orbitals were obtained with $350 B$ splines of order 15 in $\xi$ direction (covering $1 \leq \xi \leq 350 a_{0}$ ) and $24 B$ splines of order 8 along $\eta . \mathrm{H}_{2}^{+}$orbitals obtained with this basis with angular momenta $0 \leq m \leq 5$ were used to build configurations for the CI calculation of the $\mathrm{H}_{2}$ states with either ${ }^{1} \Sigma_{g}$ or ${ }^{1} \Sigma_{u}$ symmetry. About 6000 (5800) configurations were used in the CI calculations for ${ }^{1} \Sigma_{g}\left({ }^{1} \Sigma_{u}\right)$. The same energy cut off $\epsilon_{\mathrm{cut}}=40 \mathrm{a}$. u. used for DFT-SAE-TDSE was also adopted in the CI-TDSE calculations.

A pseudo SAE calculation is performed. In this case only configurations with one of the electrons occupying the lowest lying $\sigma_{g}$ orbital of $\mathrm{H}_{2}^{+}$are included in the CI calculation (see also [115] where it is discussed for atoms). Using the same orbitals as for the full CI calculation, this leads to about 4200 configurations for ${ }^{1} \Sigma_{g}$ and ${ }^{1} \Sigma_{u}$ symmetry. Although this implementation of the SAE contains correlation to a certain extent, the numerical efforts are still greatly reduced compared to the full CI approach. This approach is called SAE-CI in the following.

Table 3.1: Ionization potentials $\left(I_{p}\right)$ and (where applicable) $C_{l} \equiv C_{l, m=0}$ coefficients for $\mathrm{H}_{2}\left(R=1.4 a_{0}\right)$ obtained with different electronic structure calculations. This includes the density-functional approaches using the LB94 or the TSLDA functionals as well as the Hartree-Fock (HF), Xalpha, or configurationinteraction (CI) method.

\begin{tabular}{|c|c|c|c|c|}
\hline Method & $I_{p}(\mathrm{eV})$ & $C_{0}$ & $C_{2}$ & $C_{4}$ \\
\hline HF & 16.188 & 2.435 & 0.1073 & 0.0010 \\
HF [145] & 16.449 & 2.44 & 0.14 & \\
Xalpha [103] & 15.70 & 2.51 & 0.06 & 0.00 \\
LB94 & 15.32 & 1.146 & 0.0666 & 0.0008 \\
TSLDA & 17.40 & 0.4521 & 0.0231 & 0.0002 \\
LDA & 10.26 & & & \\
SAE-CI & 15.49 & & & \\
CI & 16.06 & & & \\
\hline
\end{tabular}

In Table 3.1 the vertical ionization potentials $I_{p}$ (at the equilibrium distance $R=$ $1.40 a_{0}$ at which all calculations in this work were performed) obtained with the different DFT functionals, the Hartree-Fock potential and the two CI calculations (full and SAECI) are listed. In order to calculate MO-ADK rates one needs also the so called $C_{l, m}$ 
coefficients [103]. In the present case of $\mathrm{H}_{2}$ only the values for $m=0$ are required and given for the independent-particle models DFT and HF. While the HF parameters found in the present approach are quite similar to the ones found with the aid of a numerical HF program [145], the DFT results differ more evidently. They are not only deviating from the HF results, but also among each other. Of course, it should be kept in mind that it is a rather well-known fact that few-electron atoms and molecules like $\mathrm{He}$ and $\mathrm{H}_{2}$ are notoriously difficult tasks for DFT. No $C_{l}$ coefficients are given for LDA, since the asymptotic behavior of the HOMO obtained with this functional differs too much from the correct one. Therefore, no senseful fit could be performed for the determination of the $C_{l}$ coefficients.

In all the calculations in this work a linear polarized laser with polarization parallel to molecular axis is used. As shown in Section 2.3, various pulse shape envelopes (Equations 2.22, 2.23, 2.24) are also implemented in the DFT-SAE-TDSE code. 


\section{Models and approximations in strong fields physics}

In this chapter, some of the models and approximations used in the strong-field physics are described. According to simple strong-field ionization models like the AmmosovDelone-Krainov (ADK) approximation [102] the ionization rate depends mostly on the ionization potential. However, it was experimentally discovered that a large number of molecules appear to be more stable in laser fields than atoms with a comparable ionization potential [146-149]. This phenomenon was termed as suppressed ionization and is later discussed in Chapter 9.

The initial theoretical study of atomic systems exposed to strong laser fields led to the Keldysh-Faisal-Reiss (KFR) theory [24, 150, 151]. The KFR theory has evolved and is now known as the strong-field approximation (SFA). This approach can alternatively be viewed as being the first term of an intense-field $S$-matrix theory (ISMT) [107]. In the strong-field approximation the ionization process is described as a transition from a fieldfree initial state to a final Volkov state. This transition neglects the Coulomb interaction between the emitted electron and the core. The inclusion or exclusion of a Coulomb correction factor and the choice of the gauge (length or velocity gauge within dipole approximation) have historically been debatable topics in the SFA implementation.

The extension of very popular models for describing atoms in strong fields (the SFA and the ADK model) to the molecular case leads to the so-called MO-SFA [108] and MO-ADK [103] models. Both the molecular extensions (MO-SFA and MO-ADK) correctly predict the occurrence or absence of suppressed ionization for a number of molecular systems. Fluorine molecule is one of the exceptions where these theories have failed [103, 108, 152]. There are, however, evident differences in the quantitative description of the predicted suppression ratios as well as their dependence on laser-pulse parameters [109]. Furthermore, the MO-SFA results are rather strongly gauge dependent, i. e. the predictions obtained in length (LG) or velocity gauge (VG) differ substantially. This includes not only a quantitative deviation (easily by 2 or 3 orders of magnitude due to the problem of correct incorporation of the Coulomb correction factor), but in some cases also qualitative ones. One possible example is the ionization rate for parallel or perpendicular orientation of a nitrogen molecule with respect to laser polarization. In $[153,154]$ it was found that velocity (length) gauge SFA predicts the perpendicular (parallel) orientation to possess higher ionization rates. The authors in [153] also established that the MO-SFA-LG provides the correct explanation of the experimental findings. However, a different result is found for MO-SFA-VG in $[155,156]$. In many other cases the existing experimental data are not sufficient to allow for a clear answer which of the proposed models (MO-ADK, MO-SFA-LG, or MO-SFA-VG) is most appropriate. Very recently, 
it was even argued that for molecules SFA-LG should be formulated in a different way than was previously done in deriving the MO-SFA-LG [157].

A common feature of the molecular strong-field ionization models mentioned so far is that they all rely on the single-active-electron approximation (SAE). Within the SAE ionization is described as a pure one-electron process in which all remaining (non-ejected) electrons act as frozen spectators. See Chapter 3 for a discussion on SAE approximation.

Lowest order perturbation theory (LOPT) can also be used to calculate the multiphoton ionization cross-section. The LOPT is not restricted by SAE approximation, but has other limitations. The intensity of the laser should not be very high. Another computational constraint that exists when using discretized basis sets for LOPT is that calculating 5 or more photon ionization cross-sections is very time consuming.

In the following sections ADK, MO-ADK and LOPT shall be described.

\subsection{ADK Model}

The ADK model [102] was derived to describe to the tunnel ionization of atoms and atomic ions in alternating electric fields. Tunnel ionization takes place when the electric field changes the atomic potential to an extent that the electron sees a very small modified potential and can tunnel through it. Figure 1.2 schematically shows how tunnel ionization takes place. The ADK model made the following assumptions :

- Only initial and final states are important, intermediate states play no role.

- $\omega \ll E_{\text {e }}$, the photon energy $(\omega)$ is very small as compared to the energy of the electron in the initial state $\left(E_{\mathrm{e}}\right)$.

- $F \ll F_{\text {at }}$, the field strength $(F)$ is far less than the atomic field $\operatorname{strength}\left(F_{\text {at }}\right)$.

In a linearly polarized electromagnetic field, probability of ionization per unit time $(w)$ from a state with energy $E_{\mathrm{e}}$, orbital number $l$, and projection $m$ is given by the expression $[158]$

$$
w=C_{n^{*} l}^{2}\left(\frac{3 F}{\pi \mathcal{E}_{0}}\right)^{\frac{1}{2}} E_{\mathrm{e}} \frac{(2 l+1)(l+|m|) !}{2^{|m|}(|m|) !(l-|m|) !}\left(\frac{2 \mathcal{E}_{0}}{F}\right)^{2 n^{*}-|m|-1} \exp \left(-\frac{2 \mathcal{E}_{0}}{3 F}\right) .
$$

Here

$$
n^{*}=\frac{Z}{\sqrt{2 E_{\mathrm{e}}}}
$$

$Z$ is the charge on the atomic residue and

$$
\mathcal{E}_{0}=\left(2 E_{\mathrm{e}}\right)^{\frac{3}{2}}
$$

The coefficient $C_{n^{*} l}$ is determined by asymptotically fitting the wavefunction in the region $\frac{1}{\mathcal{E}_{0}} \ll r$. In this region field of the atomic residue is weak and the external field can be neglected. The wave function $\Upsilon_{n^{*} l m}$ in this region coincides with the asymptotic 
wave of the free electron in the region at $r \gg \frac{1}{\mathcal{E}_{0}}$ :

$$
\Upsilon_{n^{*} l m}=C_{n^{*} l}\left(\frac{Z}{n^{*}}\right)^{n^{*}+\frac{1}{2}} \exp \left(-\frac{Z r}{n^{*}}\right) Y_{l m}\left(\frac{\mathbf{r}}{r}\right)
$$

Using Bohr-Sommerfeld quantization rule an analytical expression for $C_{n^{*} l}$ is

$$
C_{n^{*} l}=\left(\frac{2 \tilde{e}}{n^{*}}\right)^{n^{*}} \frac{1}{\sqrt{2 \pi n^{*}}}
$$

$\tilde{e}$ represents the Euler number and has the value 2.7182818284590. Using equations 4.1, $4.2,4.3$ and 4.5 , a final expression for the ionization rate which only depends on the field strength and ionization potential can be calculated. The final expression for the ionization rate is

$$
\begin{aligned}
w= & \left(\frac{3 F n^{* 3}}{\pi Z^{3}}\right) \frac{Z^{2}}{2 n^{* 2}}\left(\frac{2 \tilde{e}}{n^{*}}\right)^{2 n^{*}} \frac{1}{2 \pi n^{*}} \frac{(2 l+1)(l+|m|) !}{2^{|m|}(|m|) !(l-|m|) !}\left(\frac{2 Z^{3}}{F n^{* 3}}\right)^{2 n^{*}-|m|-1} \\
& \times \exp \left(-\frac{2 Z^{3}}{3 n^{* 3} F}\right) .
\end{aligned}
$$

In order to get yield out of the ionization rate, the Equation 4.6 should be integrated over the pulse time. The ionization rate $w$ is a function of field strength $F$ which in turn is a function of time. Thus, explicit function $w(F(t))$ represents the rate. Ionization yield can be calculated by integrating the rate over the pulse,

$$
\text { ionization yield }=\int_{\mathrm{P} u l s e} w(F(t)) \mathrm{d} t \text {. }
$$

\subsection{MO-ADK}

The MO-ADK (molecular ADK) model [103] is an extension of the atomic ADK model for the case of diatomic molecules. The model successfully predicts the suppressed ionization for some molecule-atom pairs which have nearly the same ionization potential. For a diatomic molecule aligned along the external field with field strength $F$, the ionization rate $w_{d}$ can be expressed as

$$
w_{d}(F)=\frac{B^{2}(m)}{2^{|m|}|m| !} \frac{1}{\kappa^{(2 Z / \kappa)-1}}\left(\frac{2 \kappa^{3}}{F}\right)^{(2 Z / \kappa)-|m|-1} \exp \left(-\frac{2 \kappa^{3}}{3 F}\right) .
$$

In Equation 4.8, $m$ is magnetic quantum number along the molecular axis, $\kappa=\sqrt{2 I_{\mathrm{p}}}$, $I_{\mathrm{p}}$ is the ionization potential for the valence electron. $Z$ is again the residue charge on the molecular system. The function $B(m)$ is defined as

$$
B(m)=\sum_{l} C_{l} Q(l, m)
$$


and $Q(l, m)$ is given by

$$
Q(l, m)=(-1)^{m} \sqrt{\frac{(2 l+1)(l+|m|) !}{2(l-|m|) !}} .
$$

The coefficients $C_{l}$ are obtained by fitting the molecular wavefunction in the asymptotic region. Using Equation 4.7 the ionization yield can be calculated.

\subsection{LOPT}

Lowest order perturbation theory (LOPT) can be used to calculate the ionization crosssections. The use of LOPT is only valid in the weak field limit. In the case of strong fields, the theory shall no longer be valid. Within the weak-field limit, the $N$-photon ionization width $\Gamma^{(N)}\left(\right.$ in $\left.^{-1}\right)$ is given by

$$
\Gamma^{(N)}=\sigma^{(N)}\left(\frac{I}{\hbar \omega}\right)^{N}
$$

where $I$ is the intensity of the monochromatic laser field in $\mathrm{W} / \mathrm{cm}^{2}, \hbar \omega$ is the photon energy in Joules, and $\sigma^{(N)}$ is the generalized cross section in the units of $\mathrm{cm}^{2 N} \mathrm{~S}^{N-1}$ and is given by

$$
\sigma^{(N)}=2 \pi \frac{\alpha c}{a_{0}} \frac{g(\omega)}{F_{0}^{N}} \sum_{f_{s}} P_{i}^{(N)}(E)
$$

where $\alpha$ is the fine structure constant, $c$ is the speed of light in vacuum $(\mathrm{cm} / \mathrm{s}), a_{0}$ the Bohr radius $(\mathrm{cm}), g(\omega)$ is a function of the photon frequency in Hartree $E_{\mathrm{h}}\left(1 E_{\mathrm{h}}=\right.$ $27.211 \mathrm{eV})$, whose definition is $\left(\omega / E_{\mathrm{h}}\right)^{N}$ for length gauge and $\left(E_{\mathrm{h}} / \omega\right)$ for the velocity gauge, $F_{0}$ is the atomic unit of photon flux $\left(F_{0}=3.22 \times 10^{34}\right.$ photons $\left.\mathrm{cm}^{-2} \mathrm{~s}^{-1}\right)$ and $P_{i}^{(N)}(E)$ the probability for an $N$-photon transition of the molecule from initial $i$ to any final state at energy $E$. The sum over $f_{s}$ runs over all accessible final-state symmetries. The $N$-photon transition probability $P_{i}^{(N)}(E)$ is given by

$$
P_{i}^{(N)}(E)=\left|D_{f i}^{(N)}\right|^{2}
$$

where $D_{f i}^{(N)}$ is the $N$-photon dipole transition matrix element between initial state $i$ and a discretized continuum final state $f$. A discretized continuum state is obtained in the present framework where the field-free Hamiltonian is solved within a finite volume or a box. The matrix element $D_{f i}^{(N)}$ is given by

$$
D_{f i}^{(N)}=\sum_{\nu, \mu, \ldots, \zeta} \frac{G_{f \nu} G_{\nu \mu} \cdots G_{\zeta i}}{\left(E_{\nu}-E_{i}-(N-1) \omega\right)\left(E_{\mu}-E_{i}-(N-2) \omega\right) \cdots\left(E_{\zeta}-E_{i}-\omega\right)}
$$


where the sum runs over all (virtual) intermediate states. The transition matrix elements $G_{j k}$ are given by

$$
G_{j k}=\left\langle\psi_{j}|\hat{\mathrm{D}}| \psi_{k}\right\rangle
$$

where $\hat{\mathrm{D}}$ is the electronic dipole operator and $\psi_{j}$ is the electronic wavefunction. The dipole operator may be expressed in length or velocity gauge with a corresponding choice of function $g(\omega)$ as given in Equation 4.12. Taking the case of 3-photon ionization cross-

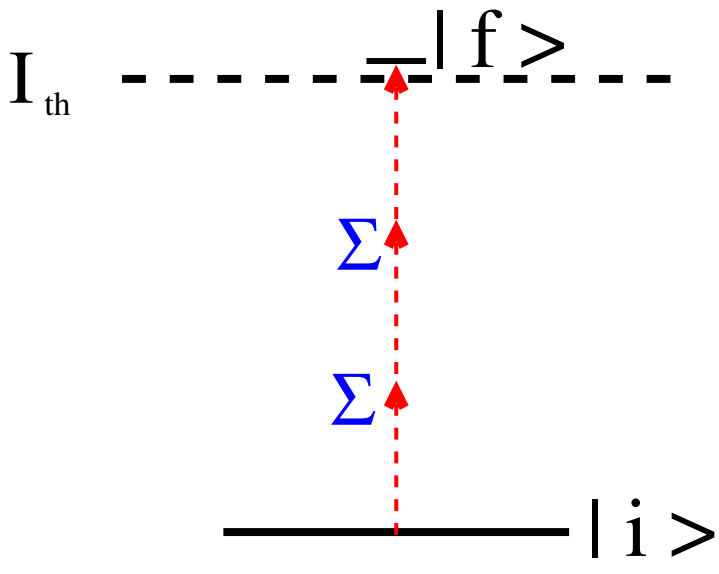

(a)

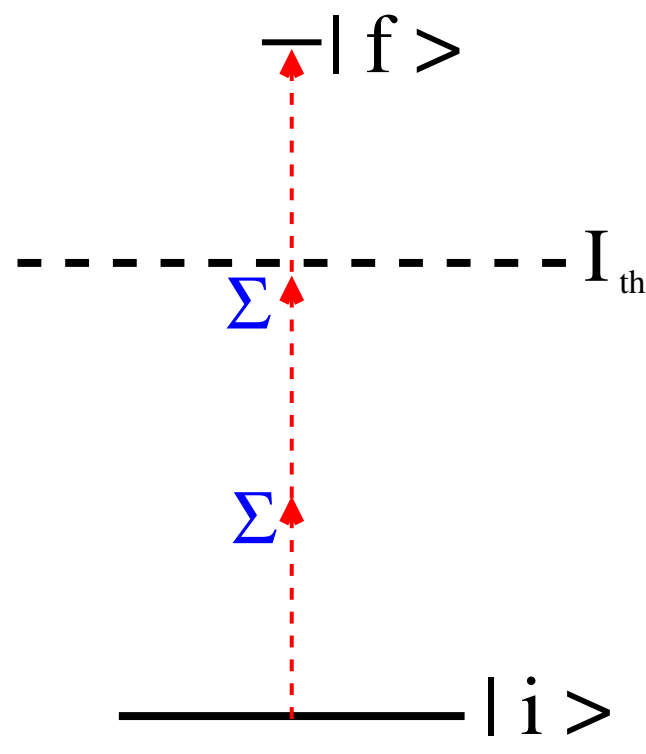

(b)

Figure 4.1: A schematic representation of 3 photon ionization. A 3-photon ionization process with minimum photon energy is shown in (a), while (b) shows the maximum photon energy that can lead to 3 photon ionization.

section when the initial state is the ground state $\left(\mathrm{X}^{1} \Sigma_{g}^{+}\right)$of $\mathrm{H}_{2}$ and laser polarization is parallel to the internuclear axis. The selection rule when the field polarization is parallel to the internuclear axis allows only the transitions between $\Sigma_{g}$ and $\Sigma_{u}$ symmetries. Since the initial state is $\mathrm{X}^{1} \Sigma_{g}^{+}$, the first virtual state, as shown in Equation 4.14 shall be of ungerade symmetry, the second virtual state shall be of gerade symmetry and the final state shall be of ungerade symmetry. Let $I_{\mathrm{p}}$ denote the ionization potential, then in the open range of photon frequencies $(\omega),\left(\frac{I_{\mathrm{p}}}{3}<\omega<\frac{I_{\mathrm{p}}}{2}\right), 3$-photon ionization shall be seen. In Figure 4.1 the schematics of three photon ionization is shown. In Figure 4.1(a) the case of the lower photon energy $\left(\omega=\frac{I_{\mathrm{p}}}{3}\right)$ is shown and Figure 4.1(b) the case of maximum photon energy $\left(\omega=\frac{I_{\mathrm{p}}}{2}\right)$ is shown. $|i\rangle$ and $|f\rangle$ denote the initial and final state, respectively. The red-dashed arrows represent a photon of energy $\omega, \sum$ symbol represents sum over all symmetry allowed virtual states. $\mathrm{I}_{\mathrm{t} h}$ represents the ionization threshold marked by black dashed lines. In the case of three-photon ionization, $|f\rangle$ 
represents all the final states with $\Sigma_{u}$ symmetry in the energy range

$$
E_{i}+I_{p}<E_{f}<E_{i}+\frac{3}{2} I_{p}
$$

where $E_{i}$ is the energy of the initial state, $E_{f}$ is the energy of the final state and $I_{p}$ is the ionization potential. The expression for the three-photon ionization cross-section starting with the ground state

$$
\sigma^{(3)}=2 \pi \frac{\alpha c}{a_{0}} \frac{g(\omega)}{F_{0}^{3}}\left|\sum_{\nu, \mu} \frac{G_{f \nu} G_{\nu \mu} G_{\mu i}}{\left(E_{\nu}-E_{i}-2 \omega\right)\left(E_{\mu}-E_{i}-\omega\right)}\right|^{2},
$$

where the index $\mu$ runs over all the states (bound and continuum) with $\Sigma_{u}$ symmetry and index $\nu$ runs over all the states with $\Sigma_{g}$ symmetry.

For each state of $\Sigma_{u}$ symmetry in the energy range as given in Equation 4.16 two summations have to be performed. If there are $\mathcal{M}$ electronic states for each $\Sigma_{g}$ and $\Sigma_{u}$ states (it is not necessary that the number of states for both symmetries to the same, it is just assumed for simplicity), then for three photon ionization cross-section $\mathcal{M}^{2}$ terms have to be summed. For an $N$ photon process there shall be $\mathcal{M}^{N-1}$ summations. Here the computation time scales as a power law with the exponent proportional the number of photons involved. Typically $\mathcal{M}$ is around 3000 to 4000 . With the increase in the number of photons, the computational time increases drastically. If we assume that $\mathcal{M}=1000$ and calculation of 2-photon ionization cross-section takes 1 second. Then 3 -photon ionization cross-section will be calculated in 1000 seconds, 4-photon ionization cross-section in $10^{6}$ seconds and 5-photon ionization cross-section in $10^{9}$ seconds. Thus, the need for computational time increases drastically as the number of photons increase. When the number of photons is large, it is better to perform the TDSE. 


\section{CI-TDSE works}

Having developed the CI-TDSE method and checked for convergence with respect to various parameters, what still remains is to verify that the CI-TDSE method actually works. In order to test that the CI-TDSE method works, it was subjected to two tests. In the first case, the internuclear distance of hydrogen molecule was reduced to a very small value $\left(R=0.001 a_{0}\right)$. Thus reducing the system to the helium atom (united atom limit). The results for full two-electron description of helium atom in laser field already exist [159-161]. In the second case, the validity of TDSE method was tested by going to the perturbative regime. The ionization cross-section for multi-photon ionization can be calculated within the lowest order perturbation theory (LOPT). The LOPT was discussed in more detail in Section 4.3. Using these ionization cross-sections along with rate equation, the ionization yield can be estimated for sufficiently long pulses.

\subsection{He: United atom limit}

In order to test the new code it was first applied to helium for which accurate theoretical results exist. For this purpose the internuclear distance was reduced to a very small value ( $R=0$ leads to a breakdown of the adopted prolate spheroidal coordinate system), since in the united-atom limit $\mathrm{H}_{2}$ corresponds to $\mathrm{He}$, if the nuclear repulsion term is omitted. The calculated energies and dipole moments became practically independent of $R$ for $R<0.001 a_{0}$. The population of continuum states (ionization yield) is shown as a function of the photon energy in Figure 5.1 for a pulse length of $3.8 \mathrm{fs}$, a $\cos ^{2}$ envelope for the vector potential $(\mathbf{A}(\mathrm{t}))$, and the peak intensity $I=2.97 \times 10^{14} \mathrm{~W} / \mathrm{cm}^{2}$. Also shown is the sum of the ionization yield and the population of all bound electronically excited states (of $\mathrm{He}$ ). This is equal to $1-P_{\mathrm{g} s}$ where $P_{\mathrm{g} s}$ is the population left in the electronic ground state of $\mathrm{H}_{2}$. These quantities have been reported (for the given laser parameters) in [161]. In that work explicitly correlated basis functions (geminals) had been adopted for calculating the field-free helium states. A good agreement is found between the two approaches, except that the present results are shifted to smaller energies with respect to the literature data. This shift is due to the inaccuracy in our ground state energy and the correspondingly underestimated ionization potential. Although it has been shown that the present code allows for very accurate ground-state calculations [123], this requires a very judicious choice of the basis set. Such a basis set (requiring for a reasonable number of $B$ splines the use of a small box) is, however, not optimal for the present purpose of describing a manifold of states, including the electronic continuum (requiring a large box). This problem is also discussed in [162] where an atomic $B$-spline based CI code is used. In fact, when comparing to the results in [162] (see Figure 5.2) there is almost perfect agreement. Besides the energy shift, the present calculation reveals all 


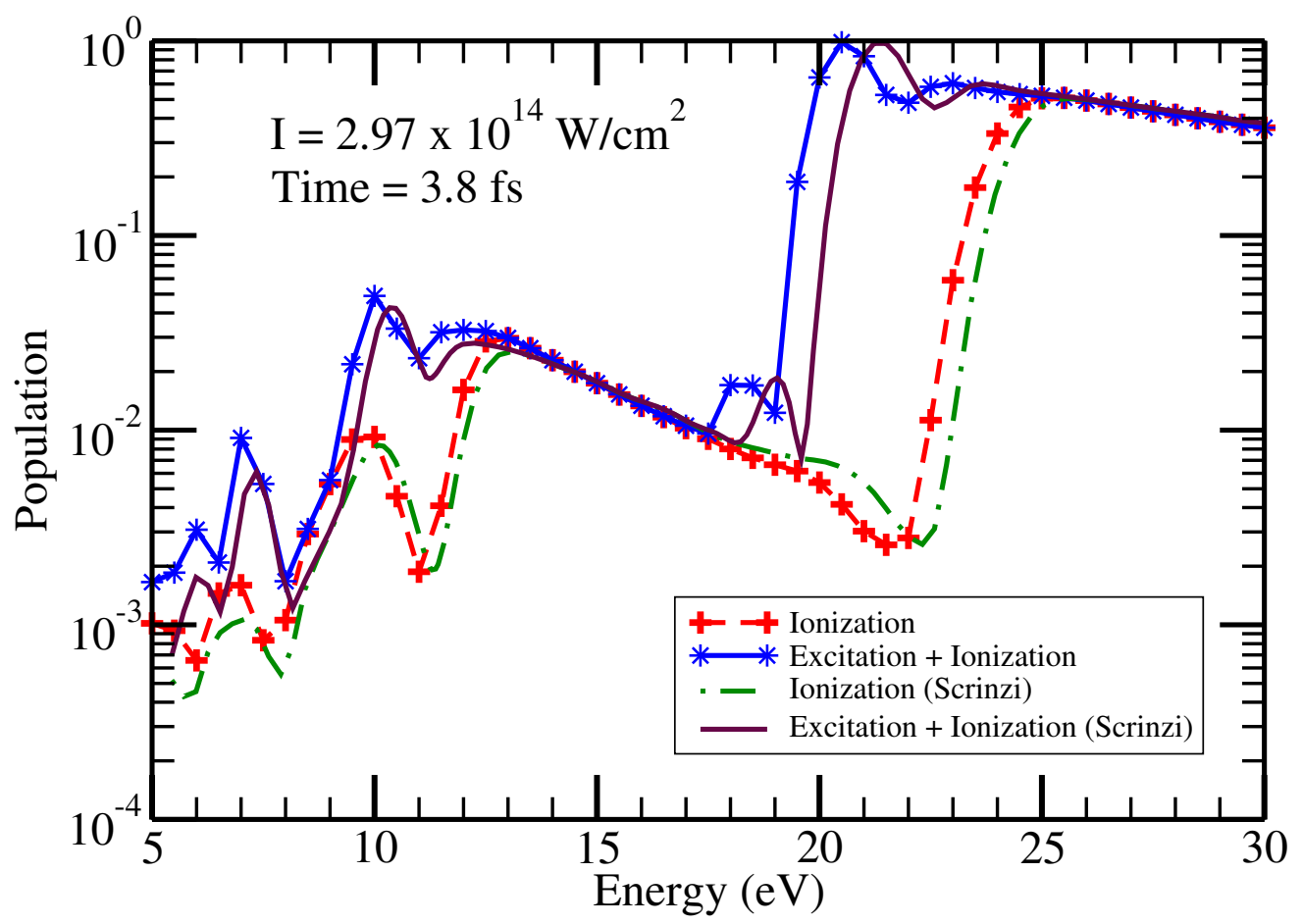

Figure 5.1: Calculated ionization yields (plus, red) and the sum of ionization and excitation to electronic bound states (stars, blue) are shown as a function of the photon energy. The calculation was performed for a very small internuclear distance $R=0.001 a_{0}$ and thus simulates atomic helium. The results are compared to the atomic calculations performed by Scrinzi et al. [161] (ionization: green chain, sum of ionization and excitation: solid maroon line.)

features found in [161], including the different multi-photon thresholds and the dip at about $20 \mathrm{eV}$ caused by Rabi oscillations.

\subsection{LOPT for He}

In order to compare the results of the TDSE calculations with the ones obtained within LOPT, it is required to convert the rates or generalized cross-sections of LOPT to excitation or ionization yields. As discussed in Section 4.3, the ionization rate $\Gamma^{(N)}$ in LOPT may be obtained from the generalized $N$-photon ionization cross-section $\sigma^{(N)}$ using Equation 4.11. Integration of the rate over the temporal pulse envelope, as already shown for ADK rate in Equation 4.7, gives the ionization yield

$$
\text { Yield }_{\mathrm{ion}}=\int_{\mathrm{P} u l s e} \Gamma^{(N)} \mathrm{d} t
$$

As is apparent from Equation 4.11 inserted into Equation 5.1 the intensity dependence 


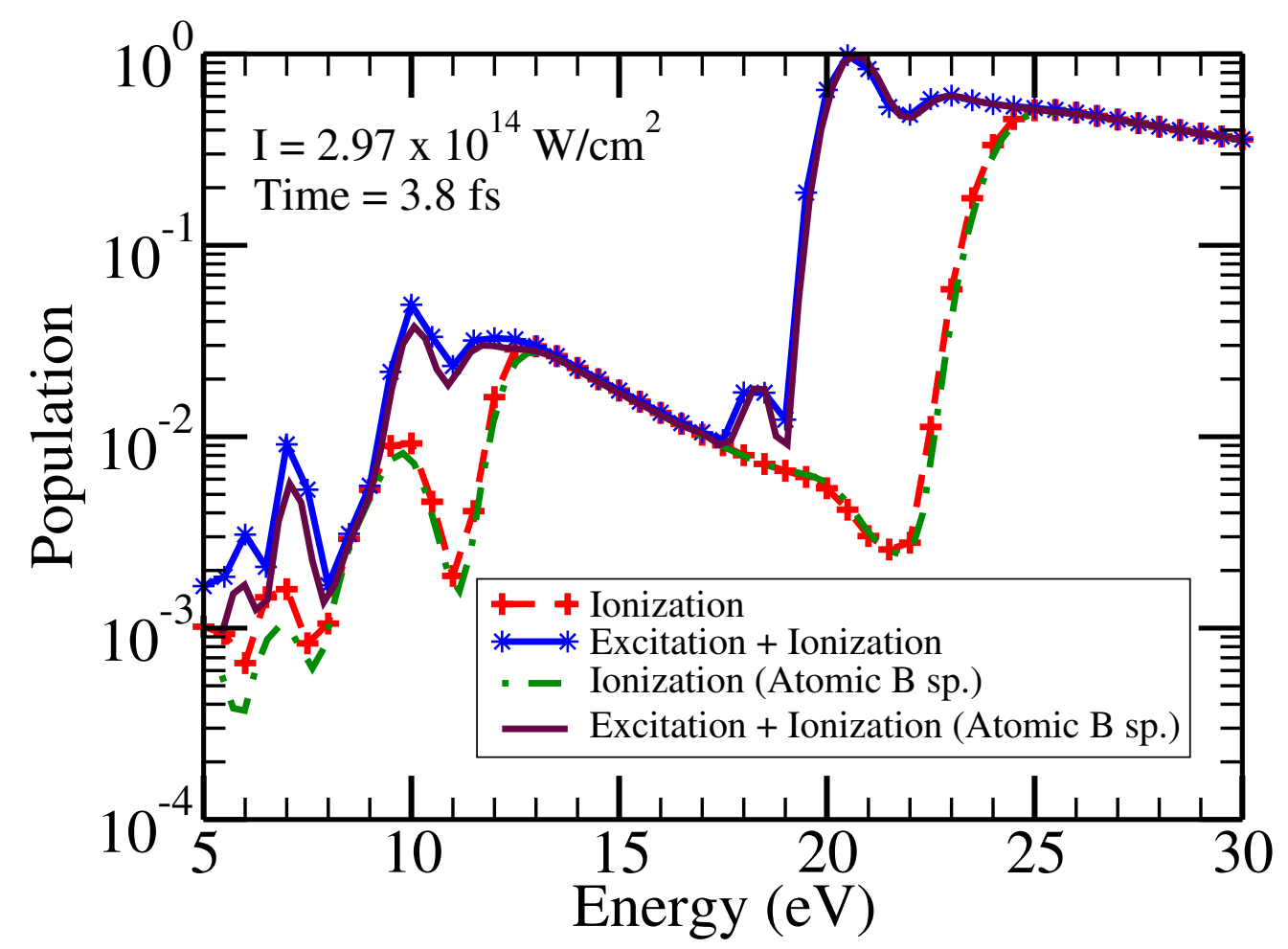

Figure 5.2: Calculated ionization yields (plus, red) and the sum of ionization and excitation to electronic bound states (stars, blue) are shown as a function of the photon energy. The calculation was performed for a very small internuclear distance $R=0.001 a_{0}$ and thus simulates atomic helium. The results are compared to the atomic calculations performed by Lambropoulos et al. [162] (ionization: green chain, sum of ionization and excitation: solid maroon line.)

of the ionization yield should be given by $I^{N}$, if the conditions for validity of LOPT are fulfilled. If the ionization yield is plotted as a function of the laser peak intensity on a double logarithmic scale, the slope should be equal to $N$, the number of photons needed for ionization. In Figure 5.3 such a plot is shown for three different photon energies. For $10 \mathrm{eV}, 6.2 \mathrm{eV}$, and $5.0 \mathrm{eV}$ photons respectively 2,3 , and 4 photons are needed for reaching beyond the ionization threshold (within the fixed-nuclei approximation). In order to achieve a comparable pulse length, the number of cycles varies for the shown photon frequencies between 20 and 36. Clearly, the intensity dependence follows in principle the one expected from LOPT. On the logarithmic plot the intensity dependence is strictly linear for a very large range of laser (peak) intensities (covering four orders of magnitude) and a change of the ionization yield by up to eight orders of magnitude. The slope of the curves is $2.00,2.93$, and 3.88 compared to the values 2,3 , and 4 predicted by LOPT. Clearly, the agreement in slope is not perfect. However, an overestimation of the slope by LOPT is a fact that was found theoretically and experimentally already for atoms (see [163] and references therein). 


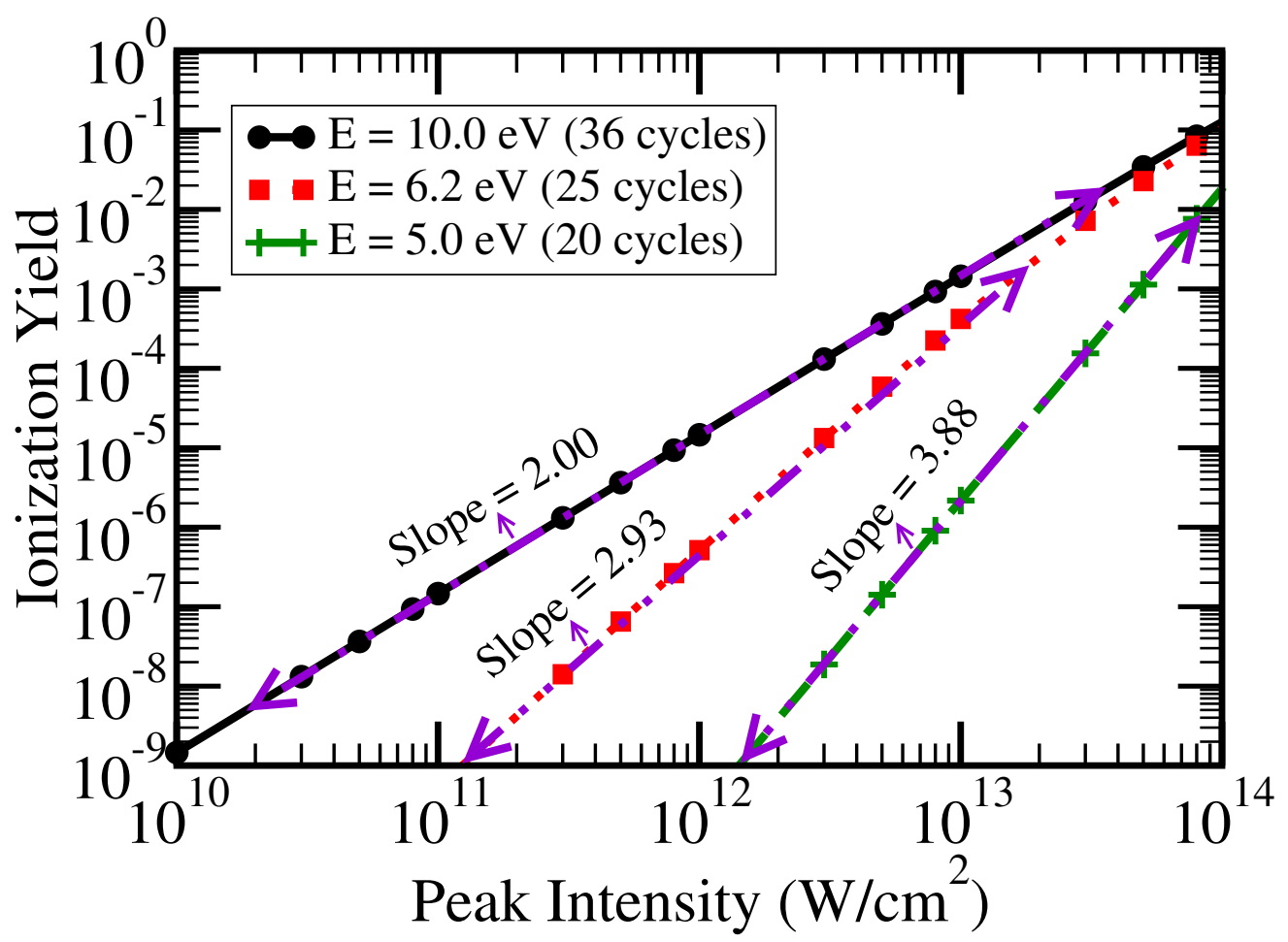

Figure 5.3: Ionization yield as a function of the laser (peak) intensity for three different photon frequencies $(10 \mathrm{eV}$ (circles, $14.88 \mathrm{fs}$ ), $6.2 \mathrm{eV}$ (squares, $16.68 \mathrm{fs}$ ) and $5.0 \mathrm{eV}$ (plus, $16.54 \mathrm{fs}$ )). According to LOPT the slope of the yield curve should be proportional to the number of photons needed for ionization, if plotted on a log-log scale. A slope of 2.0 for $10.0 \mathrm{eV}, 2.93$ for $6.2 \mathrm{eV}$, and 3.88 for $5.0 \mathrm{eV}$ is found.

In Figure 5.4 the ionization yields calculated in lowest order perturbation theory (LOPT) are compared with those obtained by a full solution of the time-dependent Schrödinger equation (TDSE). The laser-pulse parameters are given by a peak intensity of $2.0 \times 10^{12} \mathrm{~W} / \mathrm{cm}^{2}$ and a pulse length of $15 \mathrm{fs}$. A pronounced dependence of the ionization yield on the photon frequency is visible. In general, good agreement is found between the LOPT results and the full TDSE solution. At the multi-photon ionization thresholds the LOPT results are discontinuous while the TDSE calculation gives a sharp but continuous transition from one multi-photon regime (requiring $N$ photons to reach the ionization threshold) to the neighbouring ones (requiring $N \pm 1$ photons). The shown range of photon energies covers the regime from one- to four-photon ionization $(N=1$ to 4). The resonances are also more pronounced in the LOPT spectra. This is due to the finite bandwidth of the Fourier-transform limited short pulse (and possible power broadening) included in the TDSE calculation but absent in (simple) LOPT. In fact, the LOPT calculation diverges at the resonances and thus the height of the resonances calculated with LOPT is arbitrary. 


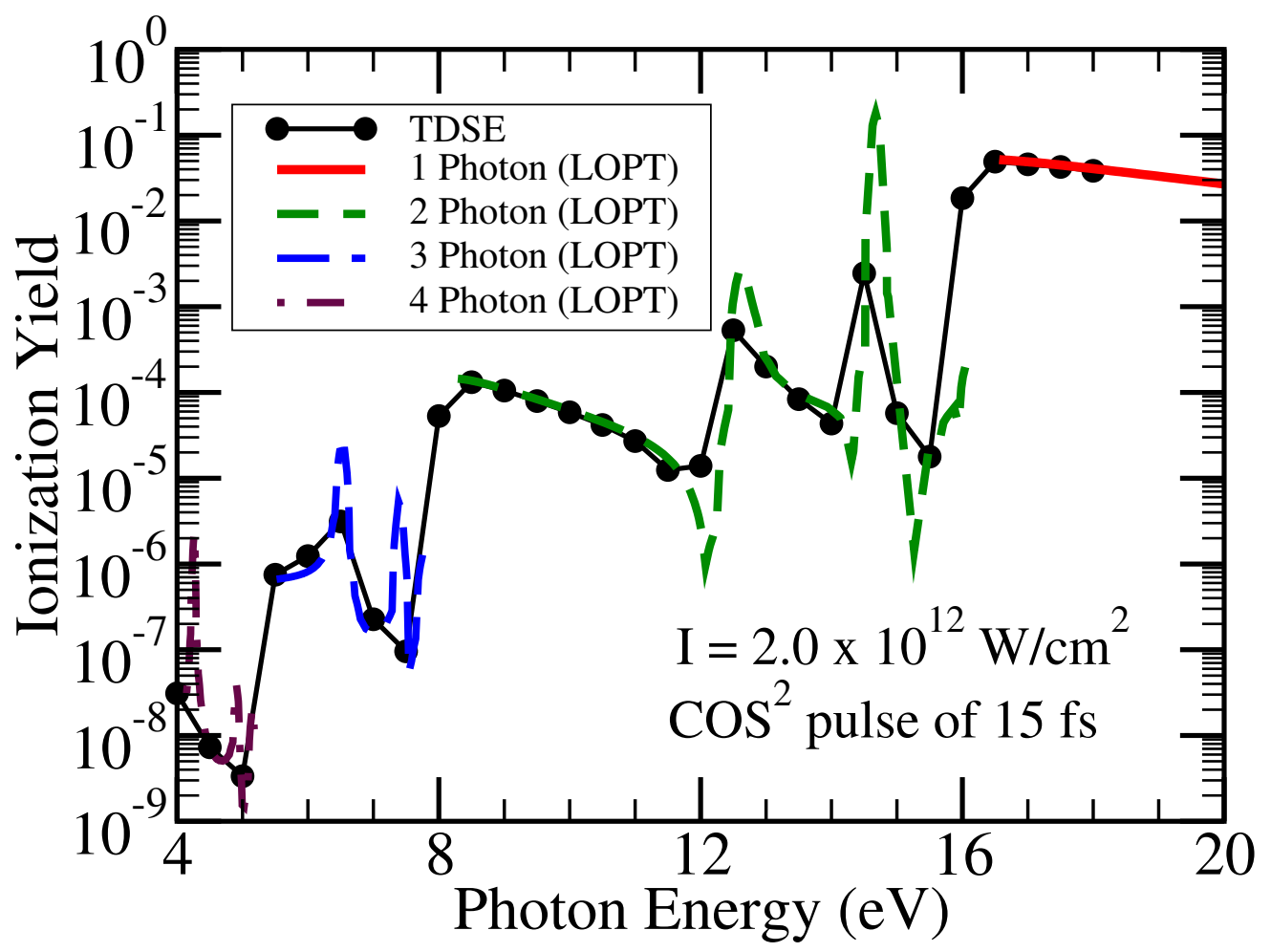

Figure 5.4: The ionization yield of $\mathrm{H}_{2}$ as a function of the photon energy for a peak intensity of $2.0 \times 10^{12} \mathrm{~W} / \mathrm{cm}^{2}$ calculated within LOPT (solid and dashed lines) is compared to the full solution of the TDSE (circles). 



\section{$6 \mathrm{CI}$-TDSE results for molecular hydrogen}

Having established that the CI-TDSE method works in the previous chapter, the TDSE for $\mathrm{H}_{2}$ molecule can be solved for various laser parameters. The results of the solution of the TDSE for $\mathrm{H}_{2}$ are presented in this chapter. The results for the photon energy scan for a fixed intensity and pulse duration are shown. The results for the popular Ti:Sa laser at $800 \mathrm{~nm}$ and its harmonics at $400 \mathrm{~nm}$ and $266 \mathrm{~nm}$ are shown. The results at these wavelengths are shown as a function of intensity as well as a function of the internuclear distance. Finally the method is used to show above threshold ionization (ATI) peaks in the photoelectron spectra. The results presented in this chapter serve as benchmarks and some of the results have been verified by other groups [118].

\subsection{Photon energy scan}

In Figure $6.1 \mathrm{a}$ the ionization yield is shown together with the sum of ionization and excitation as a function of photon energy (for the same pulse parameters as in Figure 5.4). This graph allows to discuss the spectra in more detail. For this purpose also the respective multi-photon ionization thresholds are marked (by vertical lines). The given values correspond to the results (ground-state energies of $\mathrm{H}_{2}$ and $\mathrm{H}_{2}^{+}$at the internuclear distance $R=1.4 a_{0}$ ) obtained with the present calculation. As has been discussed in the case of the helium results before, those values underestimate the correct values due to the limited accuracy of the ground state energy of $\mathrm{H}_{2}$ as obtained with the chosen basis set. It may be noted that the physical ionization threshold (beyond the present frozen-nuclei approximation) is on the other hand even smaller than the one shown.

For photon energies larger than about $16.1 \mathrm{eV}$ a single photon is sufficient for ionization. Thus the laser-molecule interaction is dominated by direct single-photon ionization without excitation of bound states. Below the one-photon threshold ionization occurs as a two-photon process. However, close to the threshold (just below $16 \mathrm{eV}$ ) two-photon ionization competes with single-photon excitation of electronic Rydberg states. The latter process is clearly dominant. At about $12.5 \mathrm{eV}$ and $14.5 \mathrm{eV}$ the ionization yield is strongly enhanced. As is evident from the summed excitation and ionization curve, the ionization is resonantly enhanced, i. e. one has a resonantly enhanced two-photon $(1+1)$ ionization process (REMPI) via the $\mathrm{B}^{1} \Sigma_{u}$ and the $\mathrm{B}^{1}{ }^{1} \Sigma_{u}$, respectively. Again, population of these excited states dominates clearly over the ionization process. For the given laser-pulse parameters the $\mathrm{B}^{1} \Sigma_{u}$ transition is in fact almost completely saturated. In between $11 \mathrm{eV}$ and the three-photon threshold at $8.0 \mathrm{eV}$ one-photon is insufficient to excite any bound state, and thus only non-resonant two-photon ionization (without excitation of bound states) is observed in this photon-energy regime. Similar to the situation at $16 \mathrm{eV}$, the ionization yield drops by more than two orders of magnitude, once the photon energy 

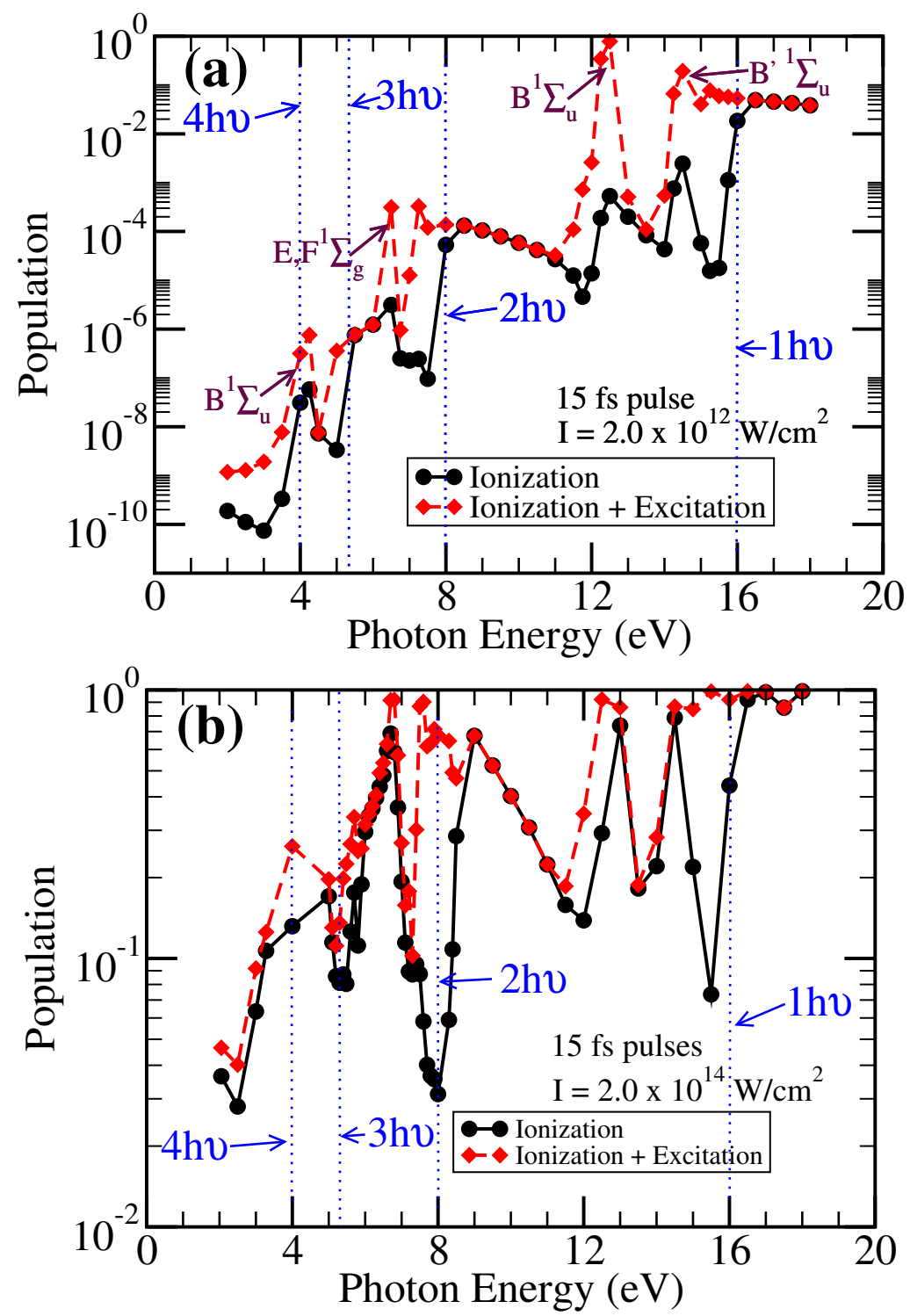

Figure 6.1: Ionization (circles) as well as sum of ionization and excitation (diamonds) for $\mathrm{H}_{2}$ as a function of photon energy for a $15 \mathrm{fs} \cos ^{2}$ pulse $(\mathbf{A}(t))$ with peak intensity $2.0 \times 10^{12} \mathrm{~W} / \mathrm{cm}^{2}$ (a) and $2.0 \times 10^{14} \mathrm{~W} / \mathrm{cm}^{2}$ (b). Ionization thresholds are marked with vertical dotted lines. In (a) some intermediate resonances are identified and marked by arrows.

is insufficient for two-photon ionization and thus three-photon ionization has to occur. The sum of ionization and excitation (and thus the depopulation of the ground state) remains (again similar to the situation at $16 \mathrm{eV}$ ) almost constant beyond the two-photon threshold. This indicates that the excitation of the Rydberg states (by two-photon absorption) occurs with a comparable probability as two-photon ionization. In view of 
the structural similarity of high-lying Rydberg states with the low-energy continuum (being also responsible for the success of quantum defect theories) this is not surprising. Around $6.5 \mathrm{eV}$ the ionization yield is again resonantly enhanced. This time a $(2+1)$ REMPI process proceeding via two-photon excitation of the $\mathrm{E}, \mathrm{F}^{1} \Sigma_{g}$ state (positioned around $13.0 \mathrm{eV}$ above the electronic ground state) is responsible. While the three-photon absorption to the $\mathrm{B}^{1} \Sigma_{u}$ state (at $4 \mathrm{eV}$ ) is clearly visible, the corresponding (3+1) REMPI process is almost hidden by the occurrence of the four-photon ionization threshold. A higher energy resolution would be required in this low photon energy regime for a more detailed discussion. However, the disappearance of pronounced structures in the low photon-energy regime is also due to physical reasons. With an increase of the number of photons involved in the absorption process, the different multi-photon thresholds approach each other. For a fixed pulse length (as in Figure 5.4) the relative bandwidth becomes thus broader. This effect tends to wash out the details of the spectrum for lower photon energies. In Figure $6.1 \mathrm{~b}$ the results obtained for the higher peak intensity $2.0 \times 10^{14} \mathrm{~W} / \mathrm{cm}^{2}$ are shown. Expectedly, the overall ionization yield is dramatically increased due to the increase of the peak intensity by two orders of magnitude. As a result of saturation and the large ionization rate of the $\mathrm{B}^{1}{ }^{1} \Sigma_{u}$ state in a high-intensity field the $(1+1)$ REMPI process dominates at around $14.5 \mathrm{eV}$. In the case of the $\mathrm{B}^{1} \Sigma_{u}$ state the situation is slightly different. While a behaviour similar to the one of the $\mathrm{B}^{1}{ }^{1} \Sigma_{u}$ state is found at the high-energy side of the resonance, excitation dominates over ionization on the low-energy side. There are two differences between the two resonant states that may be responsible for the different behaviour. Only the B state is well isolated from other (dipole-allowed) states within the bandwidth of the laser, while the B' state overlaps with the higher-lying Rydberg states. Also the ionization rate of the B' state is expected to be larger than the one of the B state, due to the difference in ionization potential. (Note, however, that it has been discussed previously [164] that these simple arguments do not necessarily apply for non-isolated excited states.) In the case of the $(2+1)$ REMPI at $6.5 \mathrm{eV}$ (E,F state) ionization and excitation is also of similar order of magnitude, the former being now dominant in contrast to the case of lower intensity.

\section{$6.2800 \mathrm{~nm}$}

Ionization and excitation (population of all excited bound states) for a $800 \mathrm{~nm}$ laser $(1.55 \mathrm{eV})$ with 12 cycles (32 fs) as a function of peak laser intensity is shown in Figure 6.2. Recently, the frustrated tunnel ionization (FTI) process was observed for He [165] at $800 \mathrm{~nm}$. Whenever the electron does not gain enough drift energy from the laser pulse after tunneling it will eventually be captured by the Coulomb field of the ion. Thus the exit channel of such process consists of neutral atoms which are very difficult to detect experimentally. Very recently, FTI was also reported for $\mathrm{H}_{2}$ molecule [166]. A study of ionization and excitation at $800 \mathrm{~nm}$, as used in experiments, can give more insight into the processes taking place. In Figure 6.2 the magnitude of ionization and excitation is almost equal, thus detection of highly excited neutral fragments comes as no surprise. A more detailed study of ionization and excitation over a large range of internuclear 


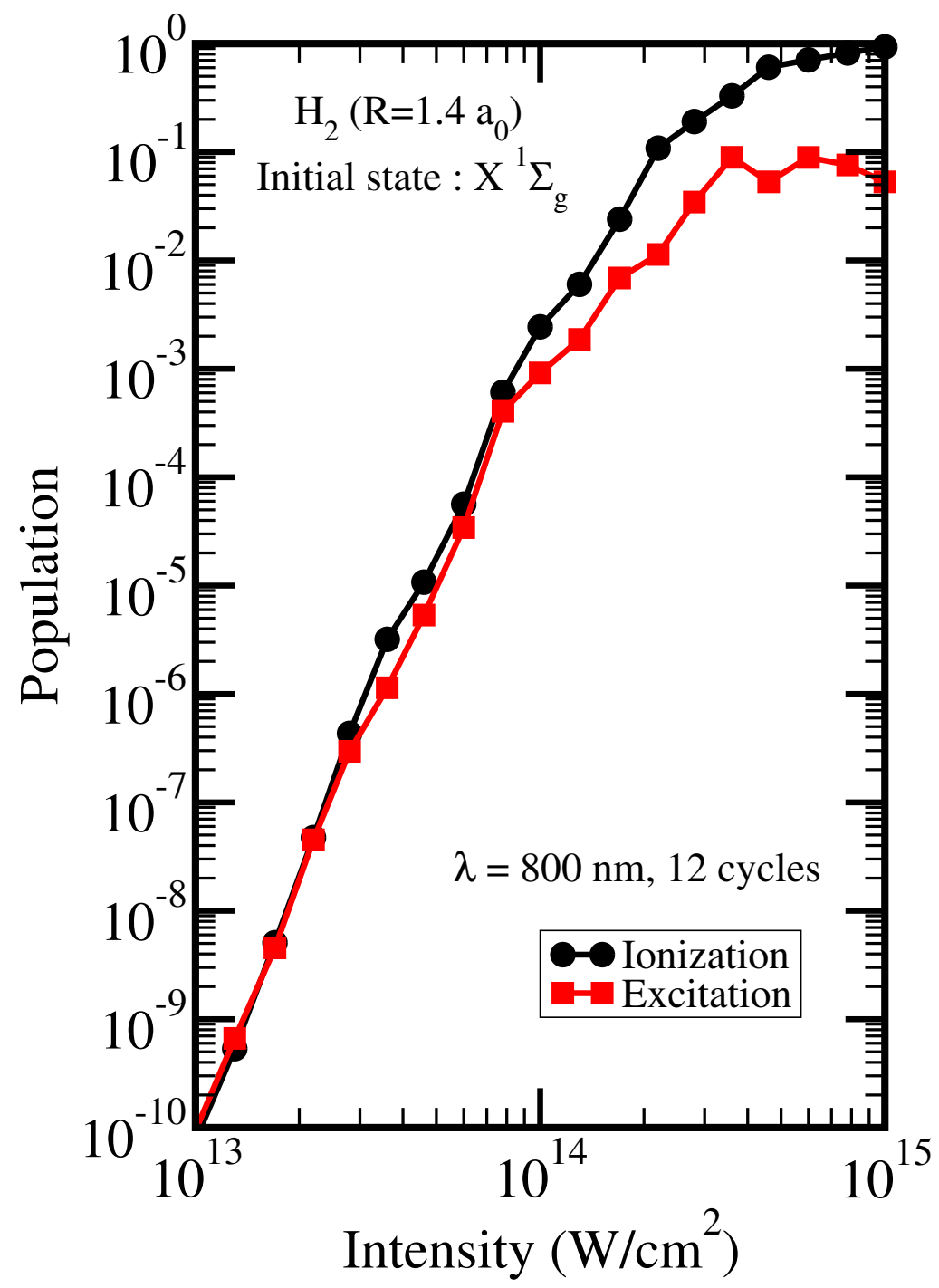

Figure 6.2: Ionization (black, circles) and excitation (red, diamonds) for $\mathrm{H}_{2}$ (at $R=$ $\left.1.4 a_{0}\right)$ as a function of laser peak intensity for a $800 \mathrm{~nm}$ laser pulse with 12 cycles (32 fs) duration is plotted.

distances is needed for a complete study and may be regarded as on the future tasks.

\subsubsection{Variation of internuclear distance}

As discussed in the previous section, in order to get the complete picture of the processes taking place when $\mathrm{H}_{2}$ molecule is exposed to laser fields the nuclear motion has to be included. The results for FTI for $\mathrm{H}_{2}$ were analyzed by using classical Monte Carlo simulations at different values of the internuclear distance [166]. When talking about the nuclear motion, Franck-Condon approximation is often used. Recently, Posthumus and 


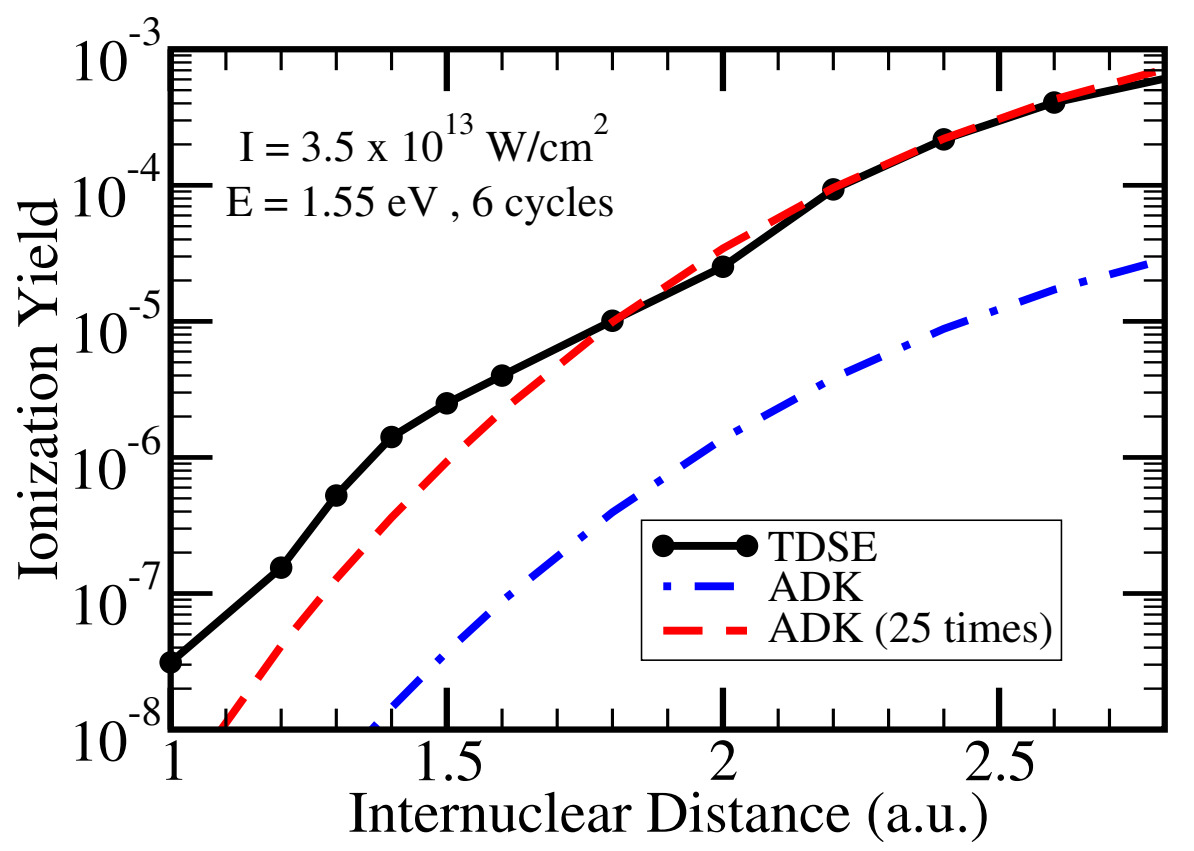

Figure 6.3: Ionization yield of $\mathrm{H}_{2}$ as a function of the internuclear distance for a laser peak intensity of $3.5 \times 10^{13} \mathrm{~W} / \mathrm{cm}^{2}$. Shown are the results for TDSE (circles) for $800 \mathrm{~nm}(1.55 \mathrm{eV})$, ADK (chain) and scaled ADK (dashed). (As is explained in the text, for the given parameters ADK agrees with the full $a \mathrm{~b}$ initio QSA prediction.)

co-workers found non-Franck-Condon distribution in the vibrational population of $\mathrm{H}_{2}^{+}$ which was obtained after ionization from $\mathrm{H}_{2}$ [105]. This surprising finding was explained on the basis of strong dependence of ionization rate on the ionization potential, which in turn depends on the internuclear distance. The result was also explained on the basis of channel closing and focal volume averaging of the laser pulse by Madsen and co-workers [167].

The strong dependence of the ionization rate on the internuclear distance has also led to an interesting process called Lochfraß [168]. Lochfraß is the depletion of the molecular electronic ground state wave-packet in a laser field and has also been experimentally observed [169].

In order to understand the non-Franck-Condon distribution in [105], the TDSE calculations were performed for several internuclear distance values in the Franck-Condon window. In Figures 6.3 to 6.6 the ionization yields obtained from the TDSE calculations for laser pulses with a pulse length of 6 cycles and a central wavelength of $800 \mathrm{~nm}$ are shown as a function of the internuclear separation $R$. The $R$ range is chosen to span the interval where the field-free initial vibrational wavefunction of $\mathrm{H}_{2}$ is localized (FranckCondon window). The peak intensity varies between $I=3.5 \times 10^{13} \mathrm{~W} / \mathrm{cm}^{2}$ (Figure 6.3) and $1.06 \times 10^{14} \mathrm{~W} / \mathrm{cm}^{2}$ (Figure 6.6). This covers the intensity range of the experiment in [105] and the chosen peak intensities agree with the ones used in the theoretical anal- 
6 CI-TDSE results for molecular hydrogen

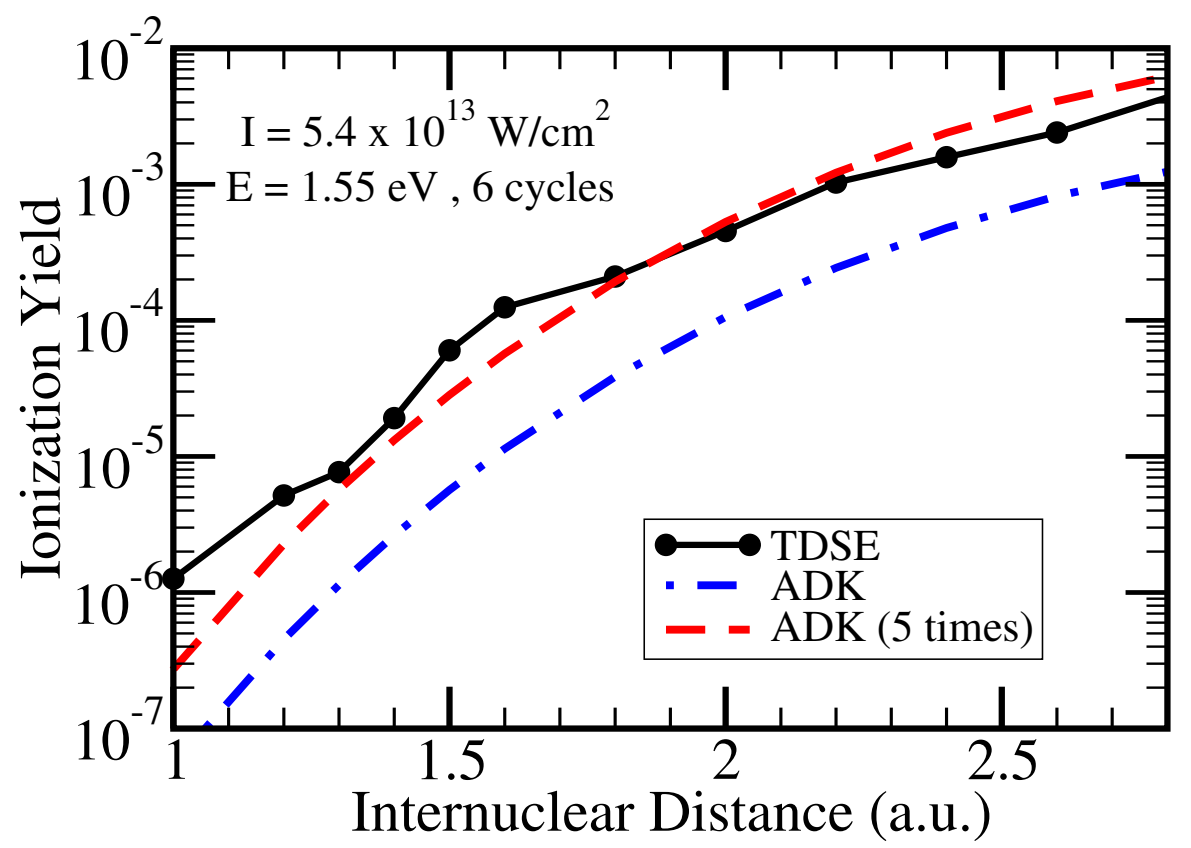

Figure 6.4: As Figure 6.3, but for a peak intensity of $5.4 \times 10^{13} \mathrm{~W} / \mathrm{cm}^{2}$.

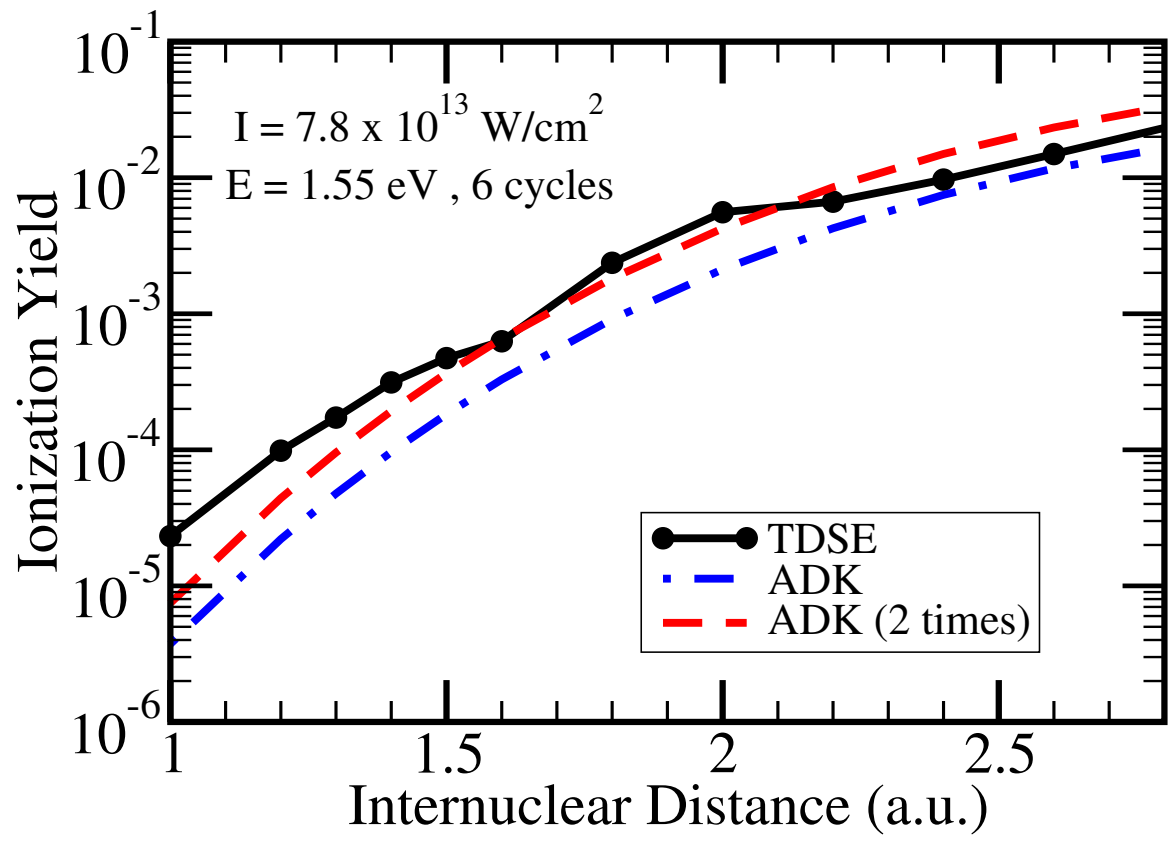

Figure 6.5: As Figure 6.3, but for a peak intensity of $7.8 \times 10^{13} \mathrm{~W} / \mathrm{cm}^{2}$.

ysis of that work. Since the ADK model (with vertical ionization potential) agrees for the given parameters very well with the corresponding static ab initio rates [56], the 


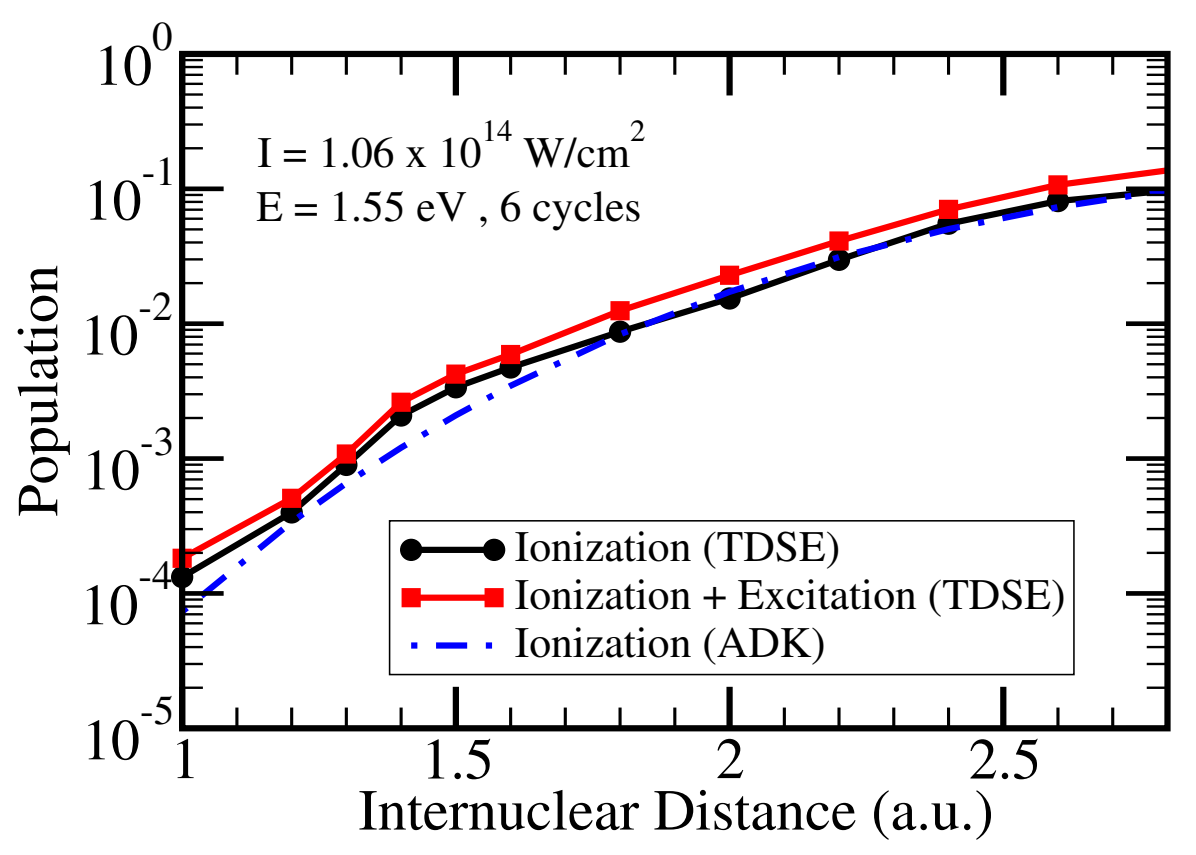

Figure 6.6: As Figure 6.3, but for a peak intensity of $1.06 \times 10^{14} \mathrm{~W} / \mathrm{cm}^{2}$. Instead of scaled ADK results the sum of ionization and population of neutral but excited $\mathrm{H}_{2}$ states (squares).

TDSE ionization yields are compared to the corresponding ADK yields obtained from the rates using Equation 4.7. For the lowest peak intensity $\left(3.5 \times 10^{13} \mathrm{~W} / \mathrm{cm}^{2}\right)$ the ADK yield underestimates the TDSE result in a drastic fashion, namely by about a factor 25 . However, as a scaling of the ADK yield by a factor 25 shows, the $R$ dependence predicted by the ADK model (using a vertical ionization potential) agrees qualitatively quite well with the TDSE result for $R>1.8 a_{0}$. For smaller $R$ values the TDSE yield shows some structure (absent in ADK) and does not rise as steeply as predicted by the ADK model. Nevertheless, in both cases the ionization yield is predicted to change by orders of magnitude within the Franck-Condon window. Thus the TDSE calculations favour the original prediction based on the strong dependence of ionization on the internuclear distance [105].

Increasing the peak intensity but keeping the other laser parameter constant leads to a clearly improved quantitative agreement between the TDSE yields and the one obtained within the ADK model. For peak intensities $5.4 \times 10^{13} \mathrm{~W} / \mathrm{cm}^{2}$ (Figure 6.4) and $7.8 \times 10^{13} \mathrm{~W} / \mathrm{cm}^{2}$ (Figure 6.5) the required scaling factor for obtaining a reasonable quantitative overall agreement decreases to 5 and 2, respectively. For the highest peak intensity considered here, $1.06 \times 10^{14} \mathrm{~W} / \mathrm{cm}^{2}$ (Figure 6.6), quantitative agreement is seen without any need for a scaling. In parallel to the improved quantitative agreement, also the qualitative agreement with respect to the $R$ dependence improves with intensity. One of the reasons for this improvement is the fact that the TDSE results become less structured when the intensity increases. Since the ADK model predicts a smooth variation 


\section{CI-TDSE results for molecular hydrogen}

of the yield with $R$, the absence of structure in the TDSE yields is a requirement for the results obtained with the two different approaches to agree to each other. The structure in the TDSE yields can be attributed to channel closing. The ponderomotive energy increases with the increase in the intensity and so do the number of photons needed for ionization. As the number of photons needed for ionization change with intensity, there occurs a channel closing. The case of $800 \mathrm{~nm}$ is not ideal for tunnel ionization for which ADK model is applicable. It is only in a very small intensity window that the conditions for tunnel ionization according to Keldysh parameter exist (see Equation 1.2). At lower intensities, multi-photon ionization takes place and structure in this regime is not surprising, while at higher intensities barrier suppression ionization takes place.

In Figure 6.6 the sum $\Sigma$ of the ionization and excitation yields is shown. Most strongfield models like the ADK or the strong-field approximation (SFA) do not include excitation. As can be seen from Figure 6.6, excitation to electronic bound states is not at all negligible for the given pulse parameters, but its $R$ dependence follows almost completely the one of the ionization yield and is also almost structureless. The comparable excitation yield signals in the direction of already discussed FTI.

\section{$6.3400 \mathrm{~nm}$}

The wavelength of $400 \mathrm{~nm}(3.1 \mathrm{eV})$ is also investigated. $400 \mathrm{~nm}$ being the second harmonic of the standard and popular $800 \mathrm{~nm}$ Titanium-Sapphire laser is also used in experiments [170,171]. For the $400 \mathrm{~nm}$ case, the results at $R=1.4 a_{0}$ and also as a function of the internuclear distance will be shown.

In Figure 6.7 ionization and excitation as a function of intensity are shown. 24 cycles of $400 \mathrm{~nm}(3.1 \mathrm{eV})$ laser are used. This corresponds to a pulse length of $32 \mathrm{fs}$. The ionization curve almost shows a smooth behaviour, increasing monotonically with intensity. The excitation curve shows some kind of structure. The dip in the excitation curve at $5.0 \times$ $10^{13} \mathrm{~W} / \mathrm{cm}^{2}$ is a result of channel closing. The fourth state of $\Sigma_{u}^{+}$symmetry was being resonantly populated at intensities less than $4.0 \times 10^{13} \mathrm{~W} / \mathrm{cm}^{2}$. Above the intensity of $4.0 \times 10^{13} \mathrm{~W} / \mathrm{cm}^{2}$ the ponderomotive energy shift becomes significant and the fourth state of $\Sigma_{u}^{+}$symmetry can no longer be resonantly populated by five photon transition to the state.

The ionization curve in the low intensity regime (below $5.0 \times 10^{13} \mathrm{~W} / \mathrm{cm}^{2}$ ) on log-log scale is quite close to a straight line, with a slope of 5.6. Using Equation 4.11 given in formulation of LOPT (Section 4.3), one can say that a 6 photon process is taking place and the conditions for multi-photon ionization are satisfied. Thus, this wavelength becomes very interesting as a transition from multi-photon ionization (low intensity) to field ionization (high intensity) may be seen.

\subsubsection{Variation of internuclear distance}

The experiment to measure the vibration distribution in $\mathrm{H}_{2}^{+}$was also performed at $400 \mathrm{~nm}$. The Keldysh condition for tunnel ionization at $400 \mathrm{~nm}$ is satisfied at very high intensities (more than $5 \times 10^{14} \mathrm{~W} / \mathrm{cm}^{2}$ ). At such intensities saturation in the ionization 


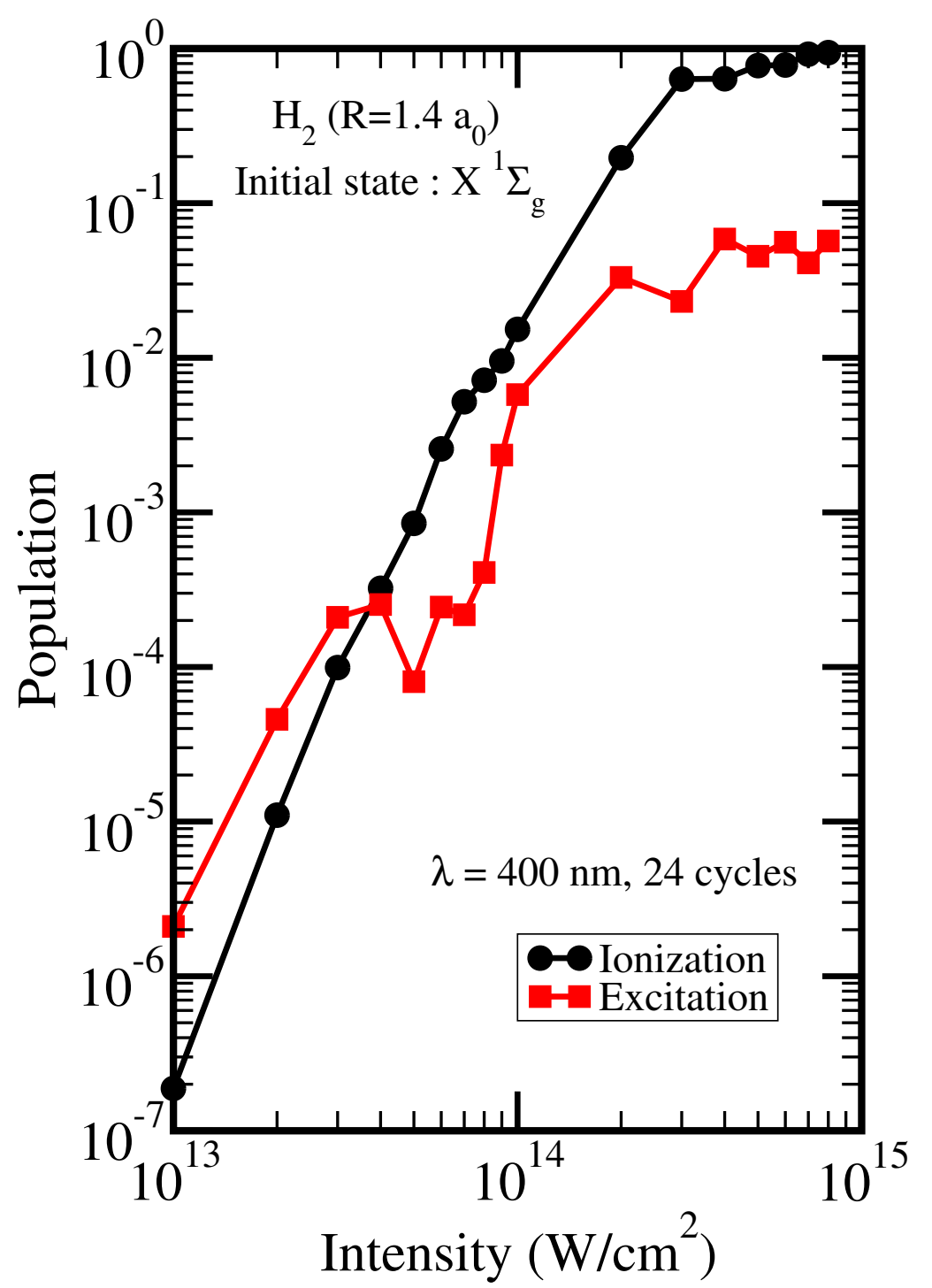

Figure 6.7: Ionization (black, circles) and excitation (red, squares) for $\mathrm{H}_{2}$ (at $R=1.4 a_{0}$ ) as a function of laser peak intensity for a $400 \mathrm{~nm}$ laser pulse with 24 cycles (32 fs) duration.

yield is reached. So one can not use the simple ADK formula for tunnel ionization to describe the ionization dynamics at $400 \mathrm{~nm}$. Thus a solution of the TDSE is needed.

In Figure 6.8 the ionization yield as a function of internuclear distance, $R$, is shown. The ionization yield is shown for four intensity values. The laser wavelength is $400 \mathrm{~nm}$ and 12 cycles(16fs) of $\cos ^{2}$ pulse for the vector potential 2.22 are used. The intensities used are $3.5 \times 10^{13} \mathrm{~W} / \mathrm{cm}^{2}$ (black, circles), $5.4 \times 10^{13} \mathrm{~W} / \mathrm{cm}^{2}$ (red, squares), $7.8 \times 10^{13} \mathrm{~W} / \mathrm{cm}^{2}$ (green, diamonds) and $1.06 \times 10^{14} \mathrm{~W} / \mathrm{cm}^{2}$ (blue, triangles). As seen in Figure 6.7 the wavelength of $400 \mathrm{~nm}$ no longer satisfies the condition for tunnel ion- 


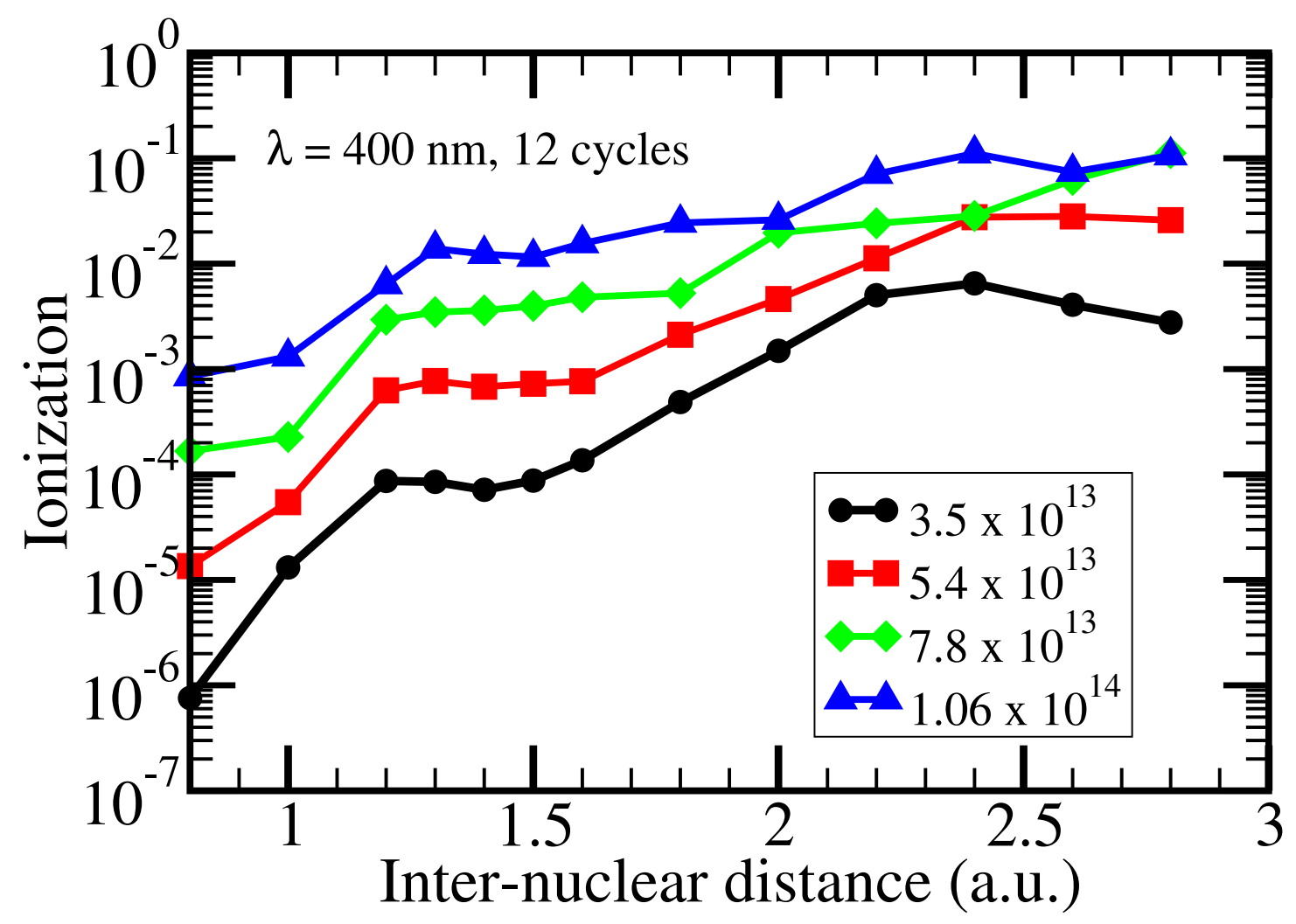

Figure 6.8: Ionization yield as a function of internuclear distance. The yields are shown for four peak laser intensities; $3.5 \times 10^{13} \mathrm{~W} / \mathrm{cm}^{2}$ (black, circles), $5.4 \times 10^{13} \mathrm{~W} / \mathrm{cm}^{2}$ (red, squares), $7.8 \times 10^{13} \mathrm{~W} / \mathrm{cm}^{2}$ (green, diamonds) and $1.06 \times 10^{14} \mathrm{~W} / \mathrm{cm}^{2}$ (blue, triangles). A $400 \mathrm{~nm}$ laser pulse with 12 cycles (16 fs) is used.

ization, so a comparison with the ADK model is not senseful.

At the intensity of $3.5 \times 10^{13} \mathrm{~W} / \mathrm{cm}^{2}$ the results for $800 \mathrm{~nm}$ and $400 \mathrm{~nm}$ show remarkable differences. At $800 \mathrm{~nm}$, as shown in Figure 6.3, a smooth increase in ionization yield as a function of $R$ is seen. At $400 \mathrm{~nm}$ the increase is no longer monotonic, the ionization yield drops at around $R=1.4$ a.u. then increases again till $R=2.4$ a.u. before decreasing again. At the higher intensities, this behaviour is not observed. At $7.8 \times 10^{13} \mathrm{~W} / \mathrm{cm}^{2}$ no decrease in ionization yield is seen. What is even more surprising is the fact that at $R=2.4$ a.u. the intensities of $5.4 \times 10^{13} \mathrm{~W} / \mathrm{cm}^{2}$ and $7.8 \times 10^{13} \mathrm{~W} / \mathrm{cm}^{2}$ give almost the same ionization yield. Thus the process of ionization shows a highly non-linear behaviour. This non-linearity is missing in simple models which often depend on a limited number of parameters like ionization potential and initial state wavefunction.

In Figure 6.9 the excitation yield as a function of inter-nuclear distance is shown. As in Figure 6.8 for the ionization yield, the excitation yields are shown for same four intensities. The laser parameters are the same as used for Figure 6.8. The excitation yields offer 


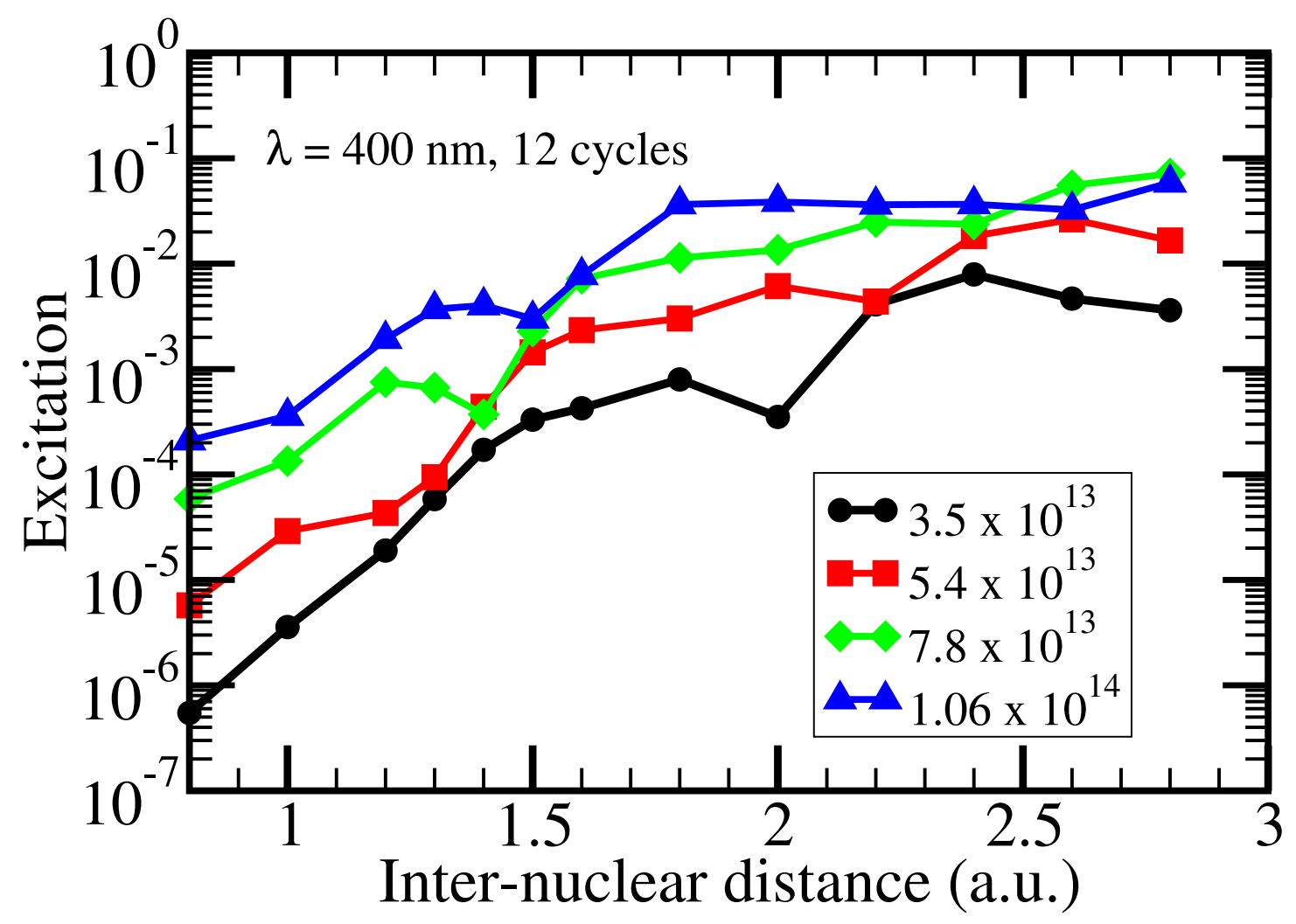

Figure 6.9: Excitation yield as a function of internuclear distance. The yields are shown for four peak laser intensities; $3.5 \times 10^{13} \mathrm{~W} / \mathrm{cm}^{2}$ (black, circles), $5.4 \times 10^{13} \mathrm{~W} / \mathrm{cm}^{2}$ (red, squares), $7.8 \times 10^{13} \mathrm{~W} / \mathrm{cm}^{2}$ (green, diamonds) and $1.06 \times 10^{14} \mathrm{~W} / \mathrm{cm}^{2}$ (blue, triangles). A $400 \mathrm{~nm}$ laser pulse with 12 cycles (16 fs) is used.

a deeper insight into the processes that are taking place. The role of intermediate bound states which are neglected in models like ADK and SFA is clearly seen. By looking at the population of the individual bound states one can see the process of channel closing. For a bound state channel closing occurs when a state being resonantly populated goes out of resonance because of the change in the ponderomotive energy. The change in the ponderomotive energy is caused by the intensity change (see Equation 1.1).

At the intensity of $3.5 \times 10^{13} \mathrm{~W} / \mathrm{cm}^{2}$, a channel closing at $R=2.0$ a.u. is seen. This channel closing comes from the sixth state of $\Sigma_{u}$ symmetry. At $R=1.6$ a.u. the sixth state of $\Sigma_{u}$ is resonantly populated. At $R=1.8$ a.u. the closely lying sixth, seventh and eighth states of $\Sigma_{u}$ symmetry are almost equally populated and contain most of the excitation. At $R=2.0$ a.u. these states are no more populated, instead of these states, now the third state of $\Sigma_{g}$ gives the maximum contribution to the excitation yield.

At the intensity of $5.4 \times 10^{13} \mathrm{~W} / \mathrm{cm}^{2}$, the dip in the excitation yield at $R=2.2$ a.u. comes from the second state of $\Sigma_{g}$ symmetry. Below $R=2.2 \mathrm{a}$.u. the second state of $\Sigma_{g}$ 
symmetry provides the dominant contribution to the excitation. While at $R=2.2$ a.u. the maximum contribution to the excitation comes from the third state of $\Sigma_{g}$ symmetry. The third state of $\Sigma_{g}$ symmetry gives the dominant contribution to excitation till $R=$ 2.6 a.u.

At the intensity of $7.8 \times 10^{13} \mathrm{~W} / \mathrm{cm}^{2}$, the first state of $\Sigma_{u}$ symmetry is responsible for the dip at $R=1.4$ a.u. The first state of $\Sigma_{u}$ symmetry dominates the excitation at $R=$ 1.2 a.u. and its contribution gradually decreases till $R=1.4$ a.u. At $1.06 \times 10^{14} \mathrm{~W} / \mathrm{cm}^{2}$ the dip at $R=1.5$ a.u. again comes from the first state of $\Sigma_{u}$ symmetry. At $R=1.5$ a.u. the contribution from the previously dominating first state of $\Sigma_{u}$ symmetry decreases and the contribution of the fourth state of $\Sigma_{u}$ symmetry starts to take over.

It is indeed very interesting to study this complicated, non-linear dynamics of the excited bound states. As seen from the analysis, the behaviour of excited bound states depends on many parameters. A slight change in the intensity, inter-nuclear distance, pulse duration or wavelength can lead to non-linear effects.

\section{$6.4266 \mathrm{~nm}$}

The wavelength of $266 \mathrm{~nm}$ corresponds to the third harmonic of the $800 \mathrm{~nm}$ laser. Thus it will be interesting to see what results the third harmonic shall give. In Figure 6.10 ionization and excitation as a function of laser intensity are shown. The results are for an inter-nuclear distance of $1.4 \mathrm{a} . \mathrm{u}$. The pulse duration is 36 cycles ( $32 \mathrm{fs})$ and $\cos ^{2}$ pulse envelope for the vector potential (Equation 2.22) is used. As seen from the figure, the slope of the ionization curve in the low intensity regime on the log-log scale is close to 4, suggesting a four-photon ionization process (see Equation 4.11).

The excitation below $5.0 \times 10^{13} \mathrm{~W} / \mathrm{cm}^{2}$ is dominated by the second state of $\Sigma_{u}$ symmetry and after that the first state of $\Sigma_{u}$ symmetry provides the dominant contribution to excitation. It should be noted that none of these states is resonantly populated.

\subsubsection{Variation of internuclear distance}

The experiment to measure the vibration distribution in $\mathrm{H}_{2}^{+}$was also performed at $266 \mathrm{~nm}$. The results at $266 \mathrm{~nm}$ can not be studied within the framework of tunnel ionization, so one needs to solve the TDSE in the Franck-Condon window of the internuclear distance.

In Figure 6.11 ionization as a function of internuclear distance, $R$, is shown. The ionization is shown for four intensity values. The laser wavelength is $266 \mathrm{~nm}$ and 18 cycles $(16 \mathrm{fs})$ of $\cos ^{2}$ pulse for the vector potential 2.22 are used. The intensities used are $3.5 \times 10^{13} \mathrm{~W} / \mathrm{cm}^{2}$ (black, circles), $5.4 \times 10^{13} \mathrm{~W} / \mathrm{cm}^{2}$ (red, squares), $7.8 \times 10^{13} \mathrm{~W} / \mathrm{cm}^{2}$ (green, diamonds) and $1.06 \times 10^{14} \mathrm{~W} / \mathrm{cm}^{2}$ (blue, triangles). Unlike the previous case at $400 \mathrm{~nm}$ as shown in subsection 6.3.1 here the behaviour of ionization is somewhat regular. The reason being that in most of the $R$ range it is multi-photon ionization and LOPT can be used. In the LOPT regime (low intensity, high photon energy; see Figure 1.1) the ionization has a simple power dependence on intensity, as given in Equation 4.11. Since for a particular $R$-value the ionization cross-section and all pulse parameters except the 


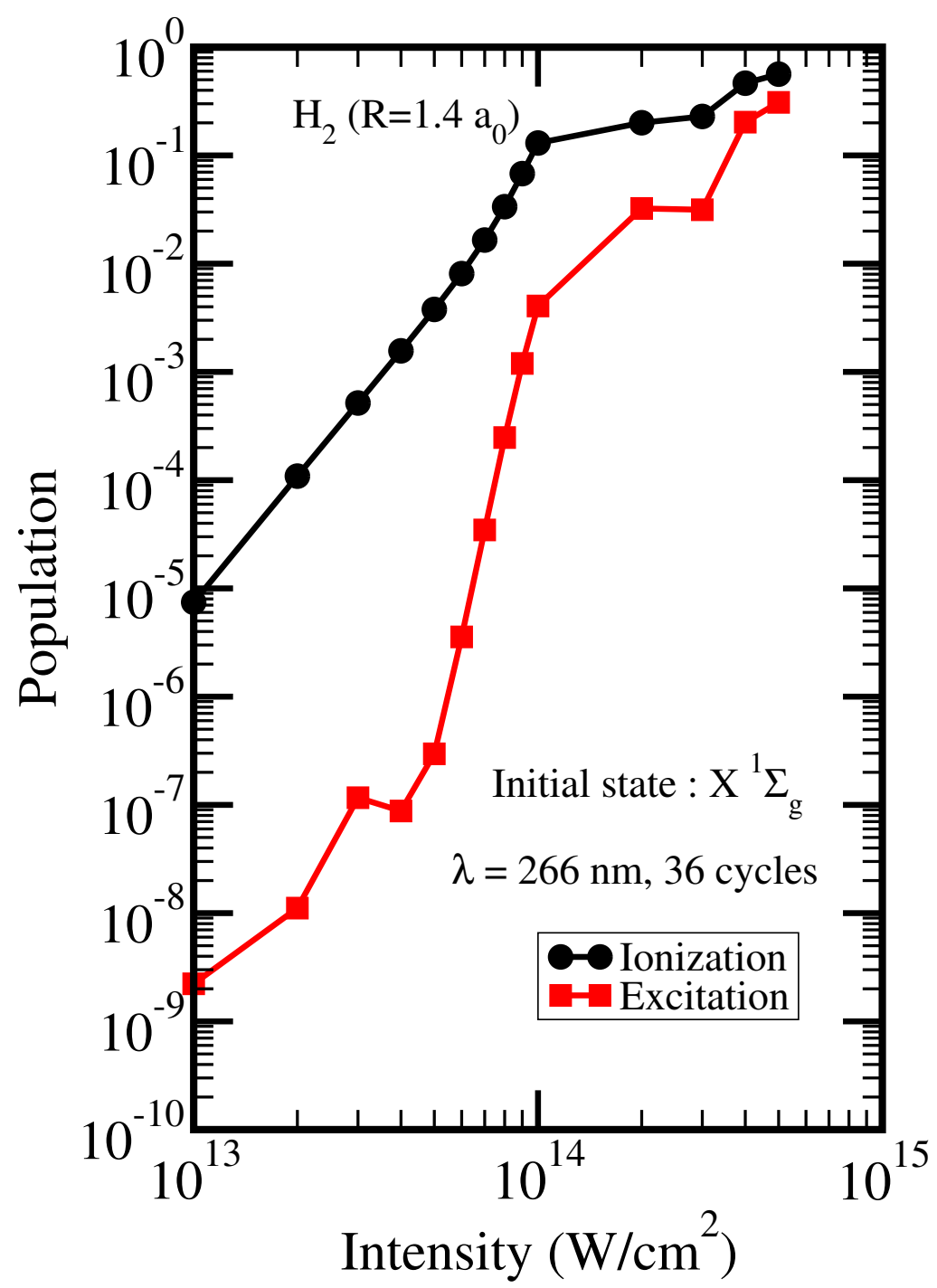

Figure 6.10: Ionization (black, circles) and excitation (red, diamonds) for $\mathrm{H}_{2}$ (at $R=$ $1.4 a_{0}$ ) as a function of laser peak intensity for a $266 \mathrm{~nm}$ laser pulse with 36 cycles (32 AFS) duration.

intensity are the same, the ionization yield has simple dependence on intensity. This simple dependence on intensity is likely to break down when the intensities are large, as shown in Figure 6.1. The ionization cross-sections for $\mathrm{H}_{2}$ molecule using LOPT already exist [106]. 


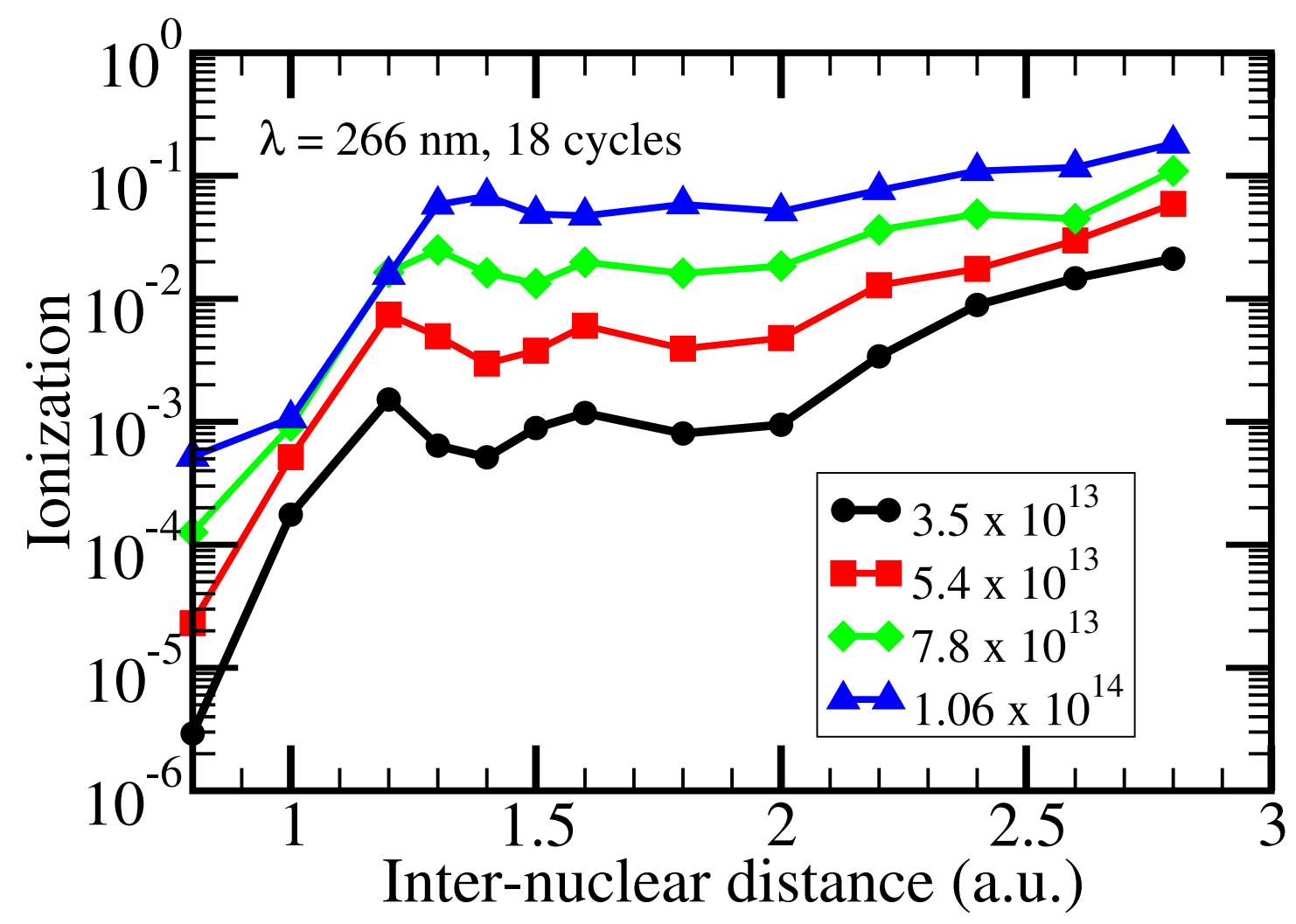

Figure 6.11: Ionization yield as a function of internuclear distance. The yields are shown for four peak laser intensities; $3.5 \times 10^{13} \mathrm{~W} / \mathrm{cm}^{2}$ (black, circles), $5.4 \times 10^{13} \mathrm{~W} / \mathrm{cm}^{2}$ (red, squares), $7.8 \times 10^{13} \mathrm{~W} / \mathrm{cm}^{2}$ (green, diamonds) and $1.06 \times 10^{14} \mathrm{~W} / \mathrm{cm}^{2}$ (blue, triangles). A $266 \mathrm{~nm}$ laser pulse with 18 cycles (16 fs) is used.

\subsection{Photoelectron spectra}

In Figure 6.12 the energy-resolved photoelectron spectra are shown for pulses with peak intensities $2 \times 10^{12} \mathrm{~W} / \mathrm{cm}^{2}$ and $2 \times 10^{13} \mathrm{~W} / \mathrm{cm}^{2}$. The photon energy is $6.0 \mathrm{eV}$ (0.22 a.u.) and the pulse contains 20 cycles. The photoelectron spectra corresponding to different symmetries $\left({ }^{1} \Sigma_{g}\right.$ and $\left.{ }^{1} \Sigma_{u}\right)$ of the complete system $\mathrm{H}_{2}^{+}+e^{-}$are given separately, but have to be added for a comparison to experiment. Within the adopted fixed-nuclei approximation the shown energy range corresponds up to about 0.8 a.u. to the situation where $\mathrm{H}_{2}^{+}$is left in its electronic ground state $\left(\mathrm{X}^{2} \Sigma_{g}\right)$. The symmetry of the complete system reflects therefore the symmetry of the photoelectron distribution. For higher energies a multichannel situation arises, since $\mathrm{H}_{2}^{+}$may also be left in one of its electronic excited states. Solutions for treating the multichannel problem (using box-discretized approaches) have been discussed in literature. As is discussed in [123], in the molecular case there exists a multichannel problem even below the first excited state of the ion. 

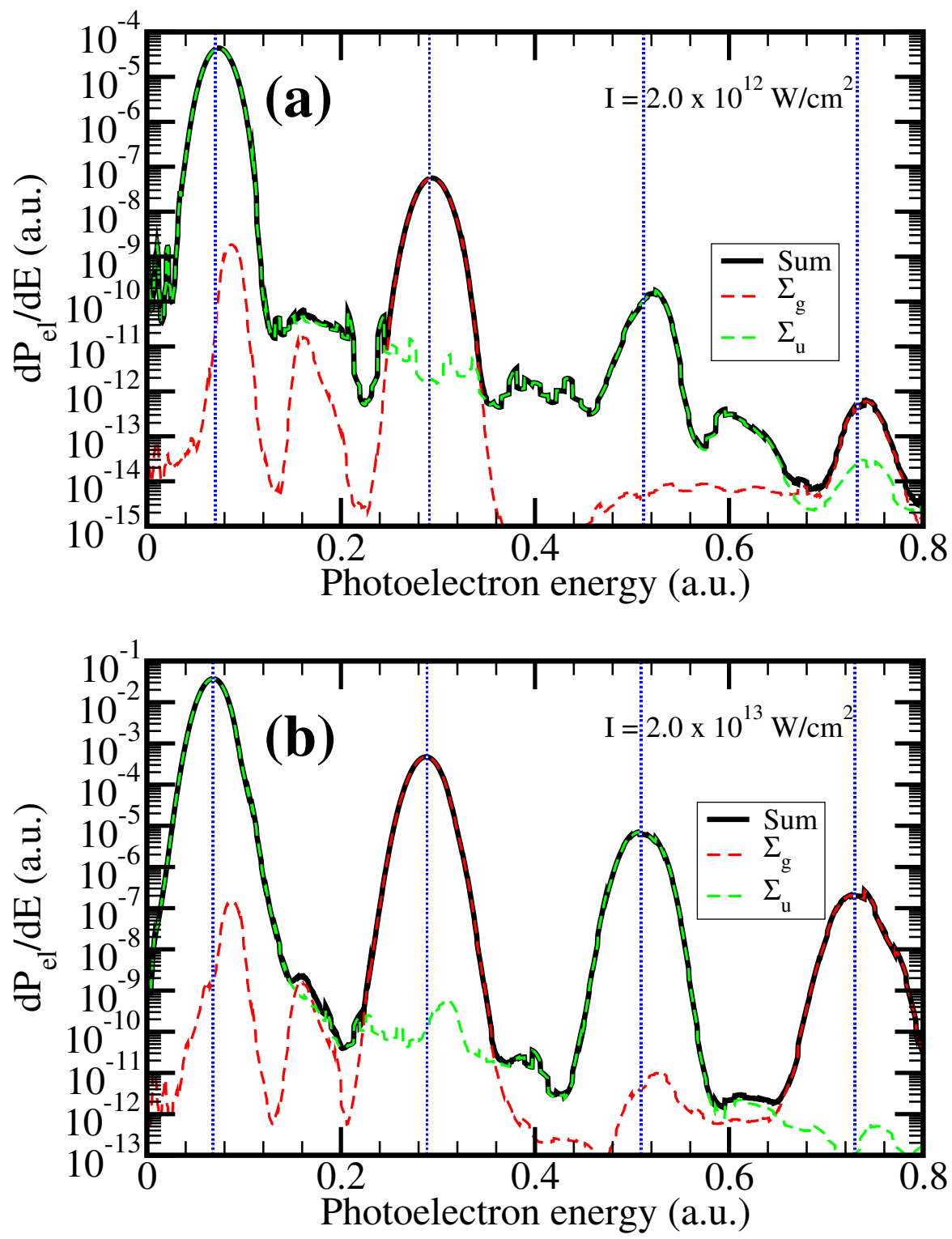

Figure 6.12: Energy-resolved photoelectron spectrum (black, solid) of $\mathrm{H}_{2}\left(R=1.4 a_{0}\right)$ after exposure to a laser pulse with photon energy $6.0 \mathrm{eV}$, length of 20 cycles, and peak intensity $2.0 \times 10^{12} \mathrm{~W} / \mathrm{cm}^{2}$ (a) or $2.0 \times 10^{13} \mathrm{~W} / \mathrm{cm}^{2}$ (b). The population of ${ }^{1} \Sigma_{g}$ (red, dashed) and ${ }^{1} \Sigma_{u}$ (green, dashed) final states are shown separately. The vertical dotted blue lines show the expected position of the photoelectron peaks.

The solution discussed there (based on an analysis of the leading channel of a given state and a subsequent density-of-states normalization) is also adopted here.

Although it has been shown above that for the pulse parameters in Figure $6.12 \mathrm{a}$ 


\section{CI-TDSE results for molecular hydrogen}

LOPT gives very accurate results for the total ionization rate, non-perturbative effects are nevertheless visible from the energy-resolved spectrum. According to LOPT a photon energy of $6.0 \mathrm{eV}$ corresponds to three-photon ionization. Therefore, the dipole selection rule allows only for final states with ${ }^{1} \Sigma_{u}$ symmetry. Figure 6.12 a shows on the other hand that the population of ${ }^{1} \Sigma_{g}$ states is small, but clearly non-zero. In fact, LOPT takes only into account the energy conserving three-photon transition and thus only the first peak. Especially the other (so-called ATI) peaks (implying the absorption of more than the minimum number of photons necessary to reach the ionization continuum) are not present in standard LOPT. The key feature for validity of LOPT with respect to the total ionization rate is the dominance of the first peak that for the laser parameters in Figure 6.12 a accounts almost exclusively for the total ionization rate. This is a good example for cases where a simpler model (here LOPT) may be able to quantitatively correctly reproduce integrated observables like the total ionization yield, but still may not be capable to describe a differential observable (here the photoelectron spectra) even qualitatively.

Increasing the intensity to $2 \times 10^{13} \mathrm{~W} / \mathrm{cm}^{2}$ results of course in a larger total ionization yield, but also the shape of the photoelectron spectrum changes. For higher intensities the relative contribution of the ATI peaks to the total ionization yield increases. While for the lower laser intensity shown in Figure 6.12 a the height of the second ATI peak is about five orders of magnitude smaller than the fundamental peak, it is only three orders of magnitude for the ten times higher laser intensity in Figure $6.12 \mathrm{~b}$. As a consequence, an increasing intensity requires to take into account an increased number of ATI peaks in order to obtain the correct ionization yield. Even the higher laser peak intensity in Figure 6.12b corresponds according to the Keldysh parameter $\left(\gamma_{\mathrm{Kel}} \approx 10\right)$ still to the multi-photon regime. The non-zero background photoelectron spectrum indicates the fact that even for the given values of Keldysh parameters there is some strong-field ionization occurring that reflects the field character of the photons. Its contribution to the total ionization yield is, however, completely negligible. 


\section{SAE-TDSE Results}

There are two main motivations for the present SAE approach. First, for large systems with many electrons full calculations of their behavior in strong laser pulses appears extremely difficult with present computational resources. An SAE approach is the first step in the direction of few-electron models to such large systems. Second, it is possible to investigate the validity of strong-field models that adopt the SAE together with additional approximations. The most prominent examples are MO-SFA [108] and MOADK [103]. The comparison to MO-ADK is made in this work and MO-SFA was shown in [172].

In a purely theoretical validation of those models it is possible to avoid the need for an averaging over a number of parameters. A comparison to experiment requires on the other hand usually averages to be performed over molecular parameters (rotational and vibrational degree of freedom and thus orientation and nuclear geometry, respectively) as well as over laser parameters like the spatio-temporal pulse profile. Furthermore, the parameters of intense laser pulses like pulse shape and peak intensity are often only known with limited precision. In view of the exponential dependence of the ionization yield on the laser intensity, an experimental uncertainty of $20 \%$ or more with respect to the peak intensity which is rather common in strong-field experiments makes quantitative comparisons difficult. As a consequence, these averagings and uncertainties can severely bias the conclusions of a comparison between theory and experiment. Finally, experiments are usually not obtaining absolute ionization yields and allow therefore only qualitative comparisons.

In the context of suppressed ionization the predictions of MO-ADK, MO-SFA-LG, and MO-SFA-VG were compared to experimental data for a number of diatomic molecules [109]. In that work it was found that MO-SFA-LG gives the overall best agreement with experiment. However, it was also concluded that the experimental data are insufficient for a clear answer. Another example for the insufficiency of experimental data to answer which gauge is more appropriate in SFA is the experimentally observed vibrational distribution in $\mathrm{H}_{2}^{+}$produced in the ionization of $\mathrm{H}_{2}$ by strong laser fields [105]. The found non-Franck-Condon distribution was first predicted on the basis of an extended atomic ADK model that takes into account vibrational motion [173]. As was shown in [105] this model gives even good quantitative agreement. However, later on both MO-SFA-LG [167] and MO-SFA-VG [174] were also shown to give good agreement with the experimental data.

Another important aspect in the validation of SAE models is their dependence on the quality of the adopted electronic structure model (for example HF vs. DFT), including basis sets etc. In the present work a consistent comparison is performed by using identical orbitals in SAE-TDSE and MO-ADK. Since the electronic structure of $\mathrm{H}_{2}$ is quite 
simple, one expects this aspect to become in fact even more important for more complicated molecular systems. Preliminary results obtained for larger molecules confirm this expectation (see Chapter 9).

\subsection{Photon-frequency variation}

Figure 7.1 shows the photon-frequency dependence of the ionization yield of $\mathrm{H}_{2}$ for a $15 \mathrm{fs}$ laser pulse and a peak intensity of $2 \times 10^{12} \mathrm{~W} / \mathrm{cm}^{2}$. For this laser intensity and the shown photon frequencies the molecular response is still almost perturbative (see Section 6.1). The displayed frequency range covers parts of the 1- to 4-photon regime. While the overall behavior agrees for all the adopted SAE models as well as for the full CI calculation, there are pronounced differences. These are due to the different excitation energies and ionization potentials $I_{p}$. The low $I_{p}$ values obtained with the LB94 functional and the pseudo SAE-CI leads to a shift of the $N$-photon thresholds to lower photon energies compared to the values found for the HF and the full CI calculation. As was demonstrated in [134] it is approximately possible to correct for the different $I_{p}$ values by a corresponding rescaling of the photon frequency.

The pronounced structure found in the 2-photon regime between about 7.5-8 and 15$16 \mathrm{eV}$ is due to resonance-enhanced multi-photon ionization (REMPI), especially through the B ${ }^{1} \Sigma_{u}$ and the B ${ }^{1} \Sigma_{u}$ states (see Section 6.1 for detailed discussion). Since the SAE models underestimate the excitation energies to these states, the REMPI peaks are shifted to smaller photon energies. The only exception is the B' ${ }^{1} \Sigma_{u}$ state obtained with the HF approach whose position agrees quite well with the CI result. In general the HF results are closer to the full CI results, since also the ionization potentials agree better.

Figure 7.2 shows the excitation yield for the same laser parameters used in Figure 7.1. The excitation yield is defined as the population left in all electronic states except the one of the initial ground state as well as the total population of the electronic continuum. Besides the resonant population of the $\mathrm{B}^{1} \Sigma_{u}$ state at about $12-13 \mathrm{eV}$ one notices a rather large excitation not only at the energy of the $\mathrm{B}^{1}{ }^{1} \Sigma_{u}$ state but also for slightly larger photon energies until the 1-photon ionization threshold. This is due to a large resonant population of Rydberg states. Similar effects are also seen at the 2-, 3-, and 4-photon thresholds.

In view of the rather pronounced frequency dependence of the ionization and excitation yields (note the logarithmic scale) it is clear that a calculation for a fixed photon frequency that does not take into account the error in the ionization and excitation energies can easily give a completely wrong result. This problem should reduce, if the non-perturbative multi-photon regime with higher laser intensities and smaller photon frequencies is considered. A typical case of interest in this regime is provided by laser pulses with the already earlier considered wavelength of $800 \mathrm{~nm}$ (corresponding to a photon energy of about $1.55 \mathrm{eV})$. 


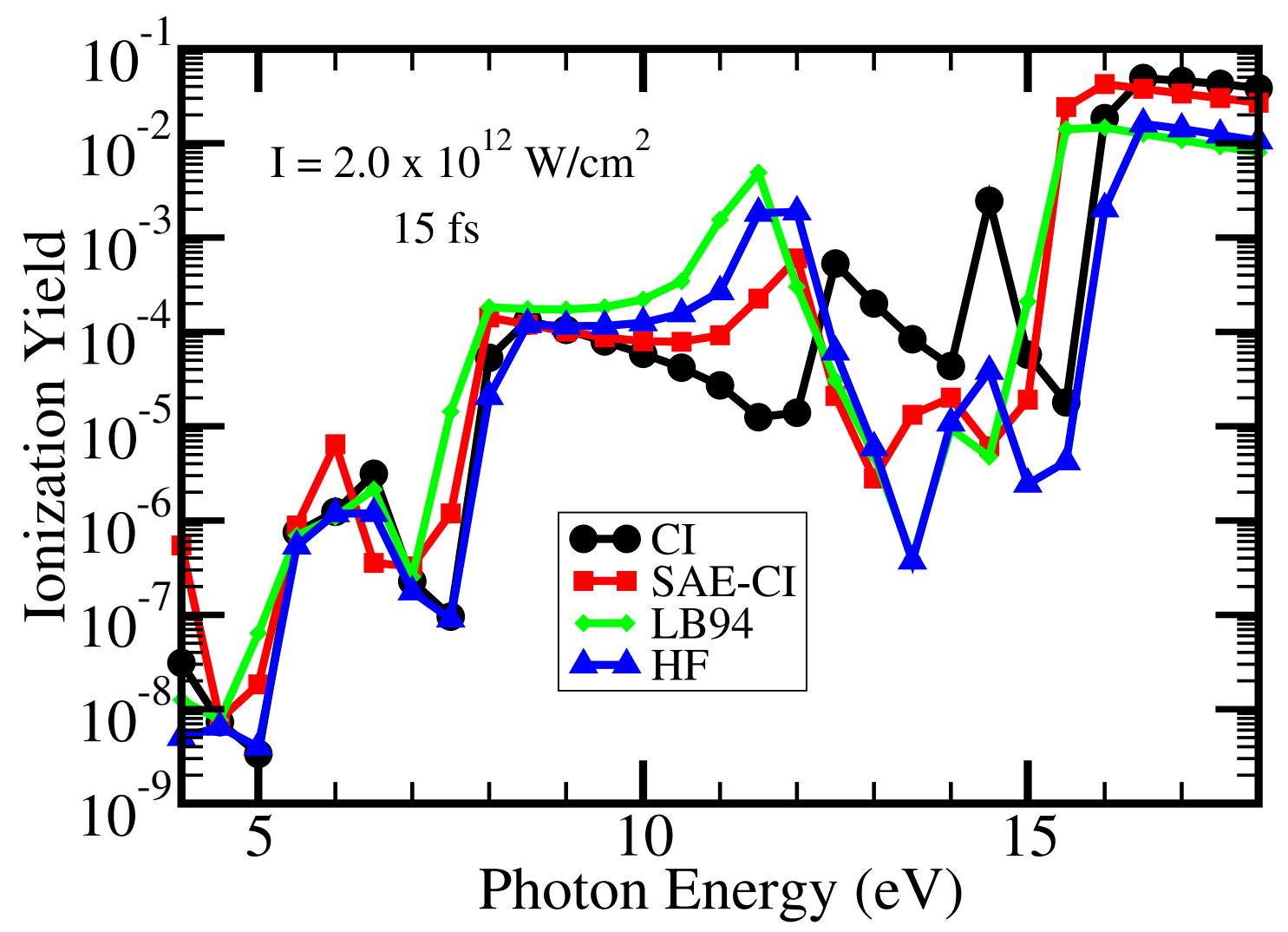

Figure 7.1: Ionization yield of $\mathrm{H}_{2}$ (at $R=1.40 a_{0}$ ) as a function of the photon energy for a linear polarized laser with parallel orientation of polarization vector and molecular axis, a peak intensity of $2.0 \times 10^{12} \mathrm{~W} / \mathrm{cm}^{2}$, and full pulse duration of $15 \mathrm{fs}$. The results obtained with the DFT-SAE-TDSE approach using either the LB94 functional (green diamonds) or HF (blue triangles) are compared to a pseudo SAE model (SAE-CI, red squares) and to a full two-electron CI (black circles) calculation.

\subsection{Intensity variation}

Figure 7.3 shows the dependence of the ionization yield on the peak intensity of a laser pulse with a central photon energy of $1.55 \mathrm{eV}$ and 12 cycles duration (corresponding to about $32 \mathrm{fs}$ ). For comparison, the results obtained from the full two-electron CI calculation are compared to the ones from the SAE using different potentials to describe the frozen core. Also the results from the SAE-CI model are plotted. Clearly, the DFT functionals LDA and TSLDA give results that differ substantially from the correct twoelectron yield. While LDA overestimates the yield (for a peak intensity of $10^{13} \mathrm{~W} / \mathrm{cm}^{2}$ by more than two orders of magnitude), TSLDA underestimates the yield by a similar order of magnitude. In addition, the slope of the curves differs. The LB94 functional that imposes a correct asymptotic long-range behavior leads on the other hand to a much 


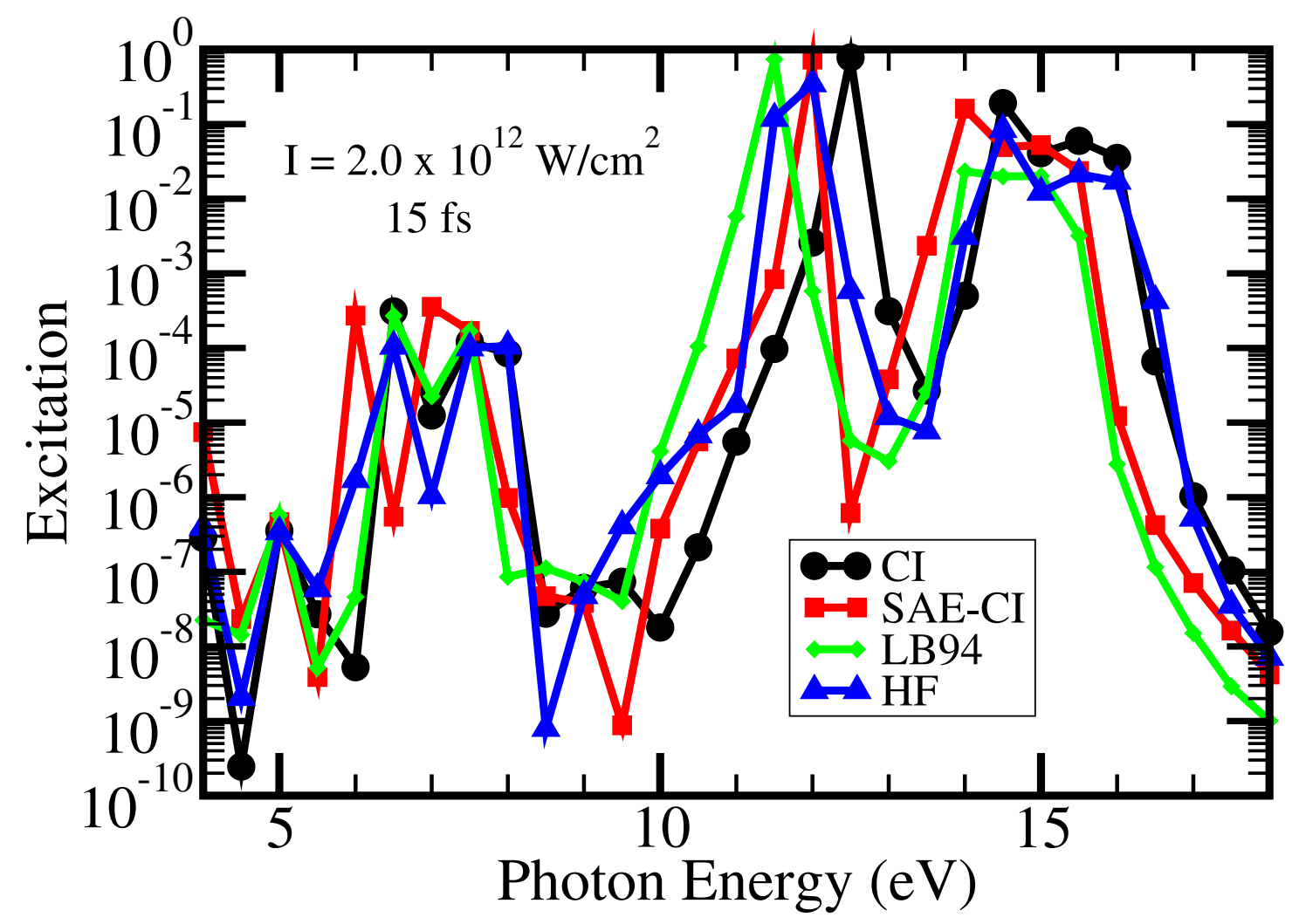

Figure 7.2: As Figure 7.1, but showing the excitation to all electronic excited bound states.

better agreement with the CI results. For peak intensities larger than $3 \times 10^{14} \mathrm{~W} / \mathrm{cm}^{2}$ the agreement is (on the logarithmic scale) even very satisfactory. The Hartree-Fock potential leads to a very similar result and in fact the two SAE results with HF or LB94 are aside from tiny local oscillations at lower peak intensities almost indistinguishable. In view of the rather different ionization potentials obtained for HF and LB94 this result is quite remarkable, since for high intensities and long wavelengths (quasi-static regime) one usually expects the ionization yield to be mainly dependent on the ionization potential, as in the case of simple ADK model. In the spirit of a multi-photon picture one may point out that within the LB94 description 10 photons are sufficient for ionization, while the HF potential requires 11 photons. A further surprise is the pronounced failure of the SAE-CI model in a large intensity range. For peak intensities between $10^{13}$ and $10^{14} \mathrm{~W} / \mathrm{cm}^{2}$ the SAE-CI model agrees surprisingly well with the LDA result. For large peak intensities (and close to saturation for the given laser pulse) SAE-CI and LDA disagree, the SAE-CI result being then much closer to the full CI result.

An intrinsic problem of the SAE is the question how equivalent electrons should be treated. In the present example of $\mathrm{H}_{2}$ there are evidently two equivalent electrons that could be ionized (within the SAE model) independently of each other. Therefore, it 


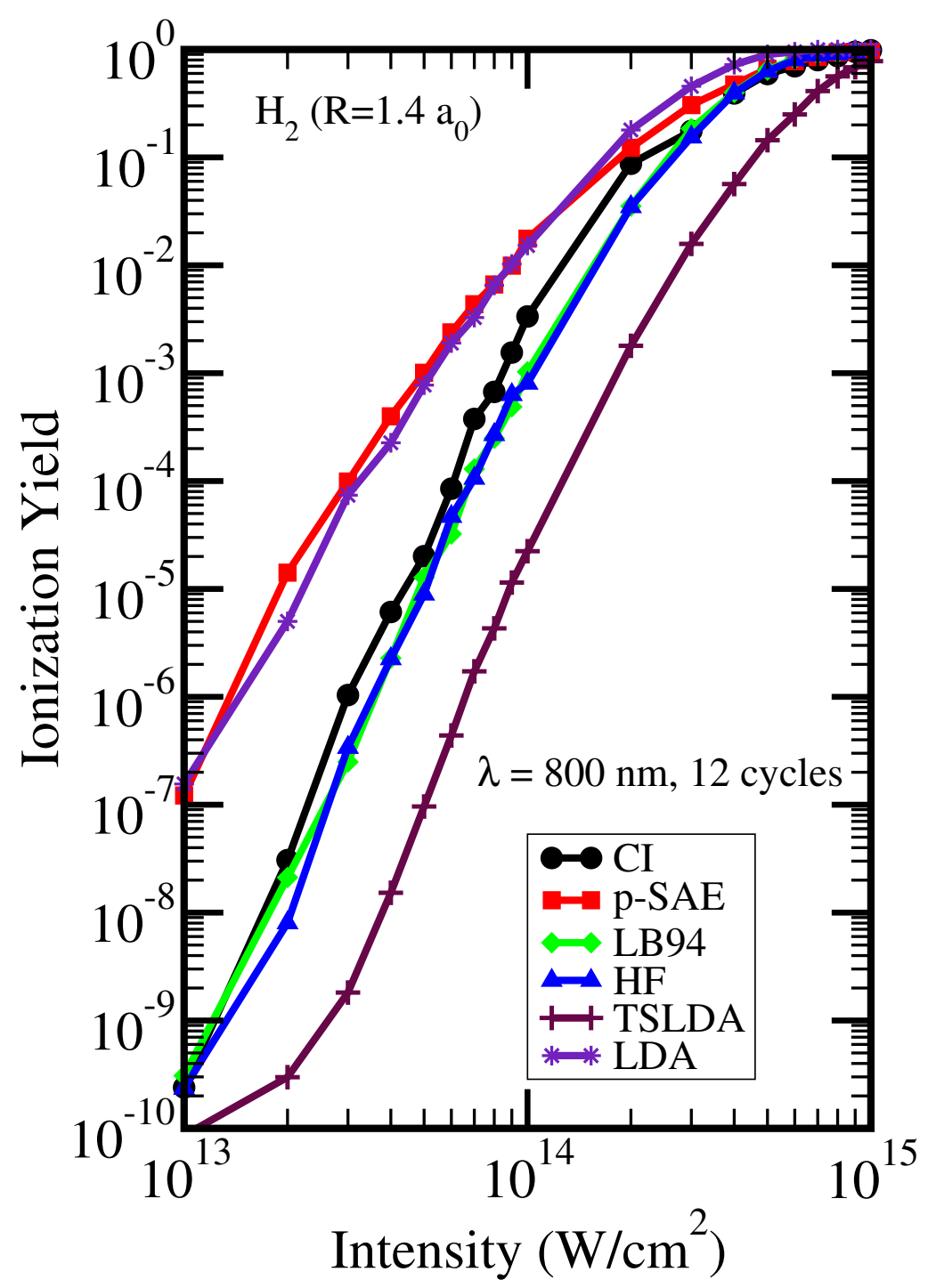

Figure 7.3: Ionization yield of $\mathrm{H}_{2}$ (at $R=1.4 a_{0}$ ) as a function of the laser peak intensity for a $800 \mathrm{~nm}(1.55 \mathrm{eV})$ laser pulse (linear polarization parallel to the molecular axis) with 12 cycles (about $32 \mathrm{fs}$ ) duration. Shown are the results obtained for full CI (black circles), SAE-CI (red squares), and the DFT-SAE-TDSE calculations using HF (blue triangles), LB94 (green diamonds), LDA (violet stars), and TSLDA (maroon crosses) functionals.

appears natural to scale the SAE yields by a factor 2. The result is shown in Figure 7.4 for the two SAE models HF and LB94 that gave reasonable agreement with the full CI result. Inclusion of the factor 2 results indeed in a very good agreement of the SAE and the CI results for laser peak intensities up to about $2 \cdot 10^{14} \mathrm{~W} / \mathrm{cm}^{2}$. At higher intensities the factor 2 leads to an overestimation of the yield by the SAE model. This 


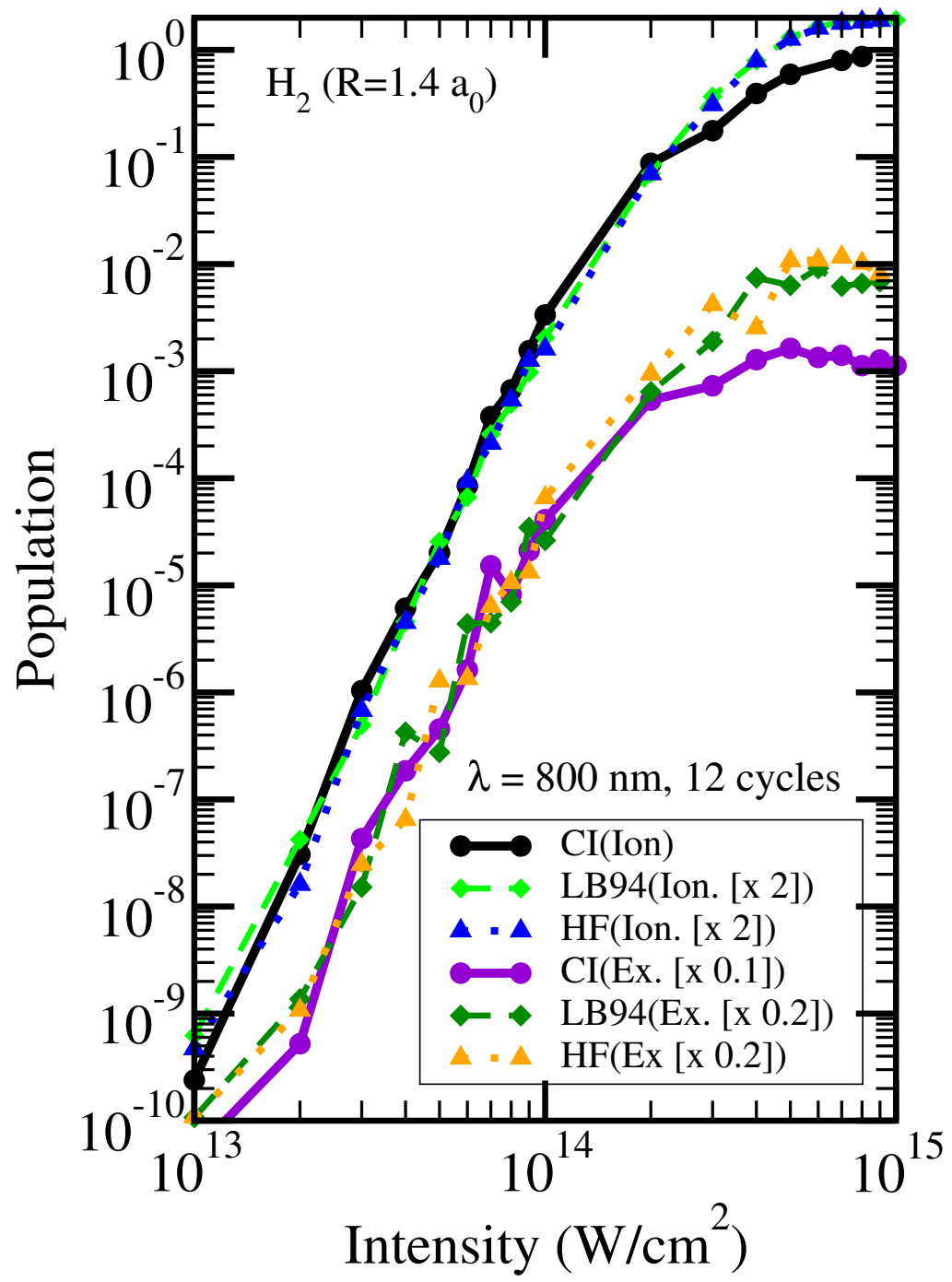

Figure 7.4: Ionization and excitation yields of $\mathrm{H}_{2}$ for the same laser parameters as in Figure 7.3. The ionization yields obtained with the DFT-SAE-TDSE calculations using HF (blue triangles) and LB94 (light green diamonds) are scaled by a factor 2 in an attempt to compensate for the two equivalent electrons when comparing to CI (black circles). Also shown are the excitation yields of CI (purple circles), HF (orange triangles), and LB94 (dark green diamonds), all scaled by a factor 0.1 for better readability and the DFTSAE-TDSE results (HF and LB94) by an additional factor 2 for the two equivalent electrons.

is expected from the good agreement found for intensities larger than $3 \cdot 10^{14} \mathrm{~W} / \mathrm{cm}^{2}$ without factor 2 in Figure 7.3. On physical grounds it is also expected that a factor 2 should not be appropriate for arbitrarily high intensities (or long laser pulses). If 
substantial ionization occurs, the remaining electron will see a less screened nuclear potential than before. Consequently, its ionization potential increases which, however, is not taken into account in the SAE approximation when adopting simply a factor 2 for the yield. From Figures 7.3 and 7.4 one can conclude that at least for $\mathrm{H}_{2}$ and $800 \mathrm{~nm}$ wavelength the SAE results should be multiplied with a factor 2 for ionization yields below $10 \%$. This is qualitatively understandable, since the change of the screening due to ionization should be small, if the ionization yield is small. The agreement of the unscaled SAE with CI for ionization yields above about $20 \%$ appears on the other hand not easy explainable and may be an accidental coincidence.

Figure 7.4 shows in addition the total excitation yield defined as the population of all electronically excited bound states. The overall agreement between the shown SAE models and the full CI calculation is again reasonably good, but especially for higher peak intensities (close to saturation) there is a clear deviation that increases with increasing laser intensity. The agreement of LB94 and HF is on the other hand in the whole intensity range very good. In view of the different excitation energies obtained with the two core potentials the agreement confirms that for $\mathrm{H}_{2}, 800 \mathrm{~nm}$ radiation, and the considered intensities the molecular response may be more ascribed to quasi-static than to multi-photon like behavior. This is further confirmed by the fact that neither the ionization nor the excitation curve shows pronounced signatures of REMPI or intensitydependent channel closings. See Chapter 1 for a discussion on quasi-static and multiphoton ionization.

Figure 7.5 shows the ionization yield for $400 \mathrm{~nm}$ wavelength (corresponding to the second harmonic of $800 \mathrm{~nm}$ ) obtained with either the CI or different SAE models. In order to maintain the same pulse duration as for $800 \mathrm{~nm}$, the number of laser cycles is doubled. The agreement of SAE-HF and CI is again quite satisfactory, but not as good as for $800 \mathrm{~nm}$. The main reason for the deviation is a channel closing occurring in the HF calculation at an intensity close to $2 \cdot 10^{13} \mathrm{~W} / \mathrm{cm}^{2}$ which is absent at this intensity in the CI result. This leads to an accidental very good agreement between the unscaled $\mathrm{HF}$ and the CI result which is reduced, if the HF yield is multiplied by a factor 2 to account for the two equivalent electrons.

The intensity at which a channel closing occurs should depend (for a given laser pulse and thus ponderomotive energy) on the ionization potential. Although this simple argument may explain why the channel closing visible at $2 \cdot 10^{13} \mathrm{~W} / \mathrm{cm}^{2}$ cannot be seen in the CI yield, the same argument would predict a completely different channel-closing intensity for the LB94 result, since the ionization potentials of the HF and the LB94 differ more than the ones of the HF and the CI calculation. On the contrary, the channel closing occurs at almost the same intensity for HF and LB94, but its consequences are only more pronounced for LB94. This leads to the observed deviation between LB94 and $\mathrm{HF}$ for intensities below $2 \cdot 10^{13} \mathrm{~W} / \mathrm{cm}^{2}$. For higher intensities the yields predicted in these two SAE models agree on the other hand very well.

A possible explanation for the good agreement of the channel-closing intensity for HF and LB94 may be the fact that its value depends not only on the ponderomotive energy that has to be added to the ejected electron in order to be ionized in the presence of a laser field. The real ionization potential in a laser field is given by the energy 


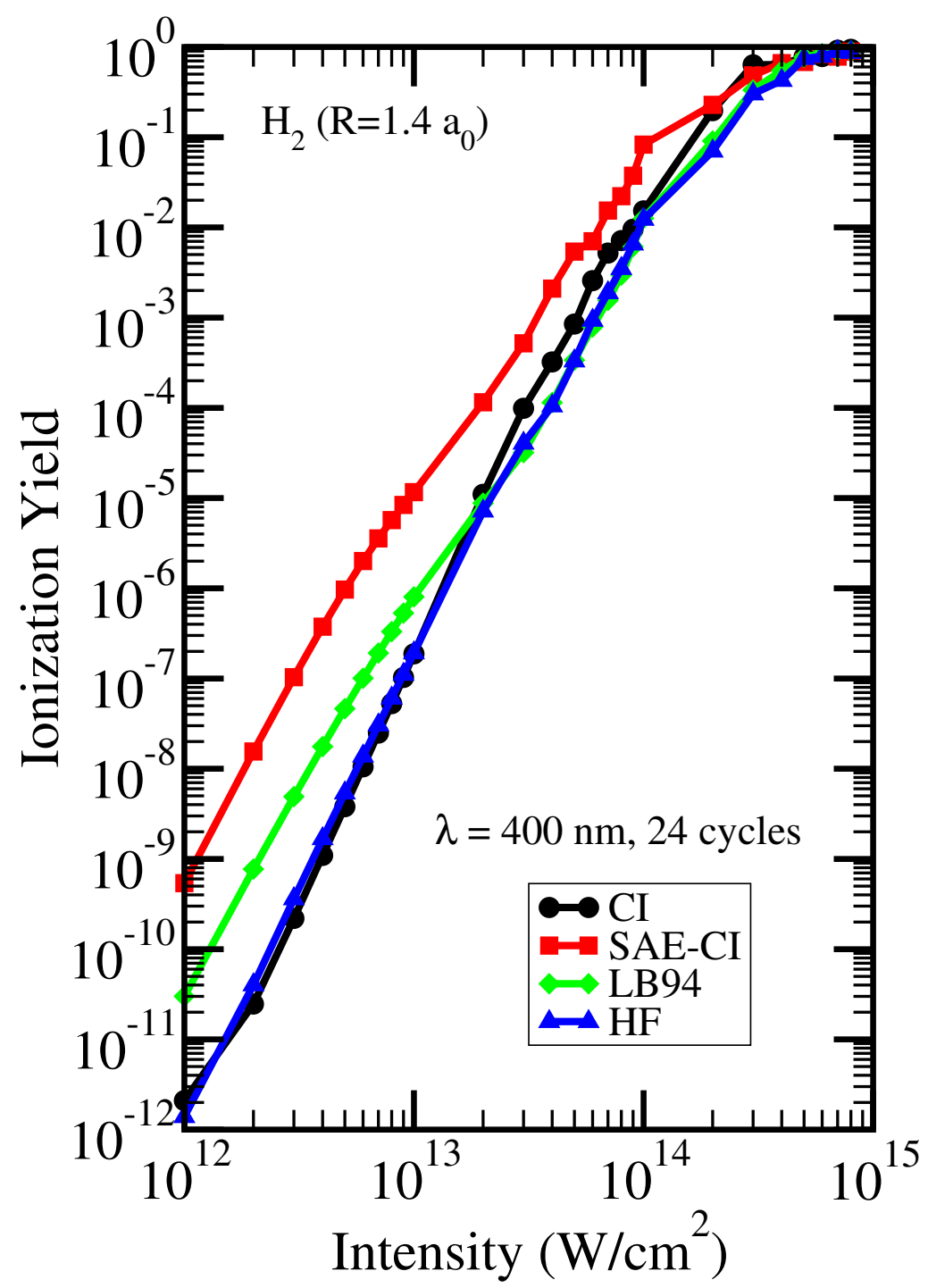

Figure 7.5: As Figure 7.3, but for $400 \mathrm{~nm}(3.1 \mathrm{eV})$ laser wavelength, full pulse duration of 24 cycles (about $32 \mathrm{fs}$ ), and omitting the results for LDA and TSLDA.

difference between the final state (ion and ionized electron with zero momentum) and the initial state (neutral molecule). Therefore, the AC Stark shift (laser-field dressing) of the initial ground state of the neutral molecule has to be considered in a determination of the ionization potential in a laser field and thus for the question at which intensity the number of photons necessary for reaching the ionization threshold increases or decreases. In view of the already discussed good agreement between LB94 and HF yields for $800 \mathrm{~nm}$ (and at higher intensities for $400 \mathrm{~nm}$ ) and thus in the quasi-static regime it appears as the difference in the field-free ionization potentials is largely compensated by the AC Stark shifts of the neutral ground state. 


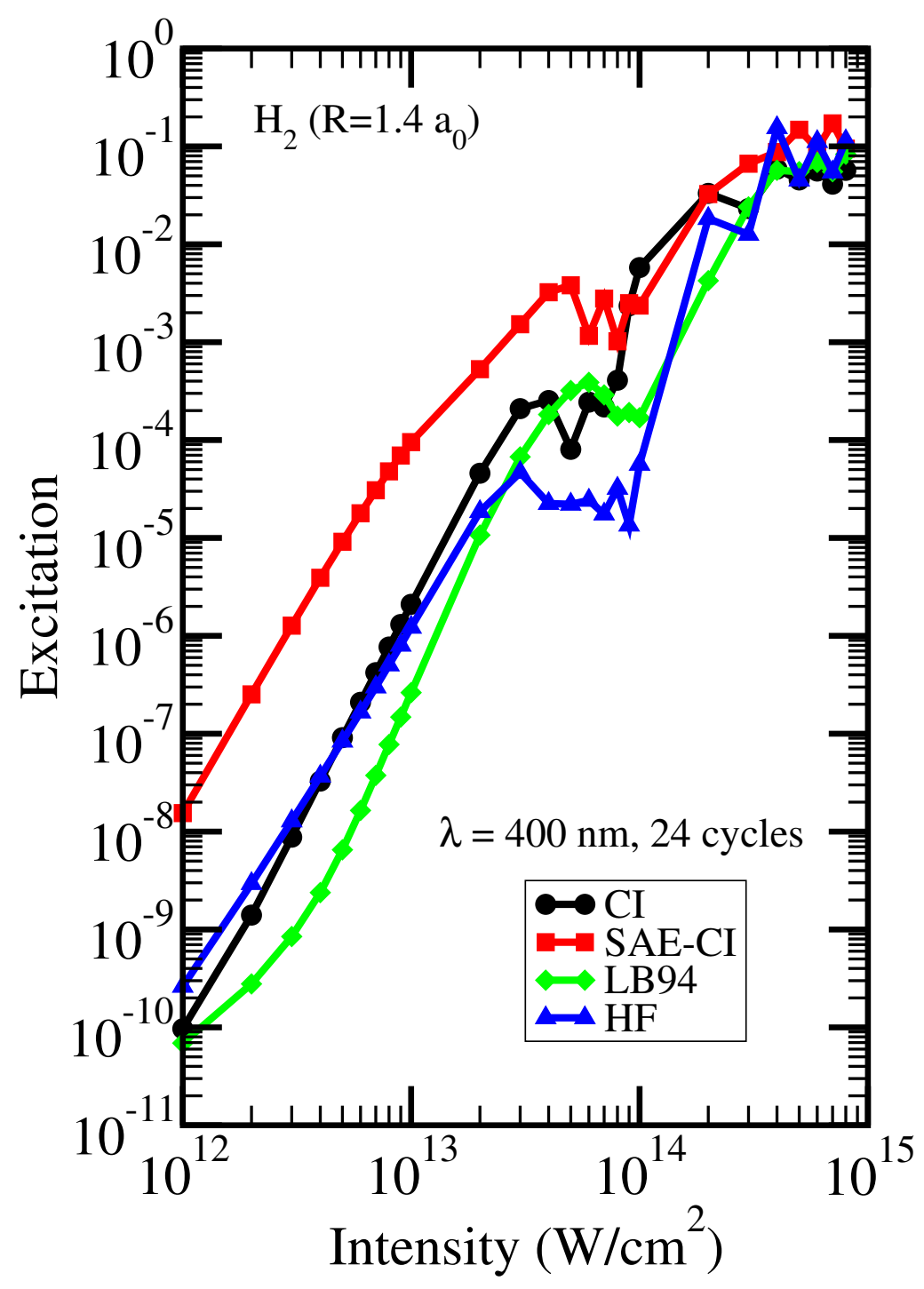

Figure 7.6: As Figure 7.5, but showing the excitation yield.

From the three shown SAE models it is again the SAE-CI approach that gives the poorest agreement with the CI yields to which it agrees best at the highest intensities. It is also remarkable that the shape of the curve follows very closely the LB94 result to which it runs (on the logarithmic scale) almost parallel for intensities below about $10^{14} \mathrm{~W} / \mathrm{cm}^{2}$. The absolute difference is, however, about one order of magnitude. The SAE-CI model overestimates also the excitation yield, as can be seen from Figure 7.6. At $400 \mathrm{~nm}$ the intensity-dependent excitation yields contain much more structure than for $800 \mathrm{~nm}$, in agreement with the fact that for this wavelength the molecular response should be more multi-photon like. The overall best agreement with the CI result is obtained with the HF functional, but LB94 still works reasonably well and actually 
yields better agreement with CI than $\mathrm{HF}$ in the intensity range between about $3 \cdot 10^{13}$ and $8 \cdot 10^{13} \mathrm{~W} / \mathrm{cm}^{2}$. Below this intensities LB94 underestimates the excitation yield. The agreement of HF to CI is on the other hand partly due to the different slopes of the two curves which leads to a crossing and thus to an accidental perfect agreement at about $4 \cdot 10^{12} \mathrm{~W} / \mathrm{cm}^{2}$.

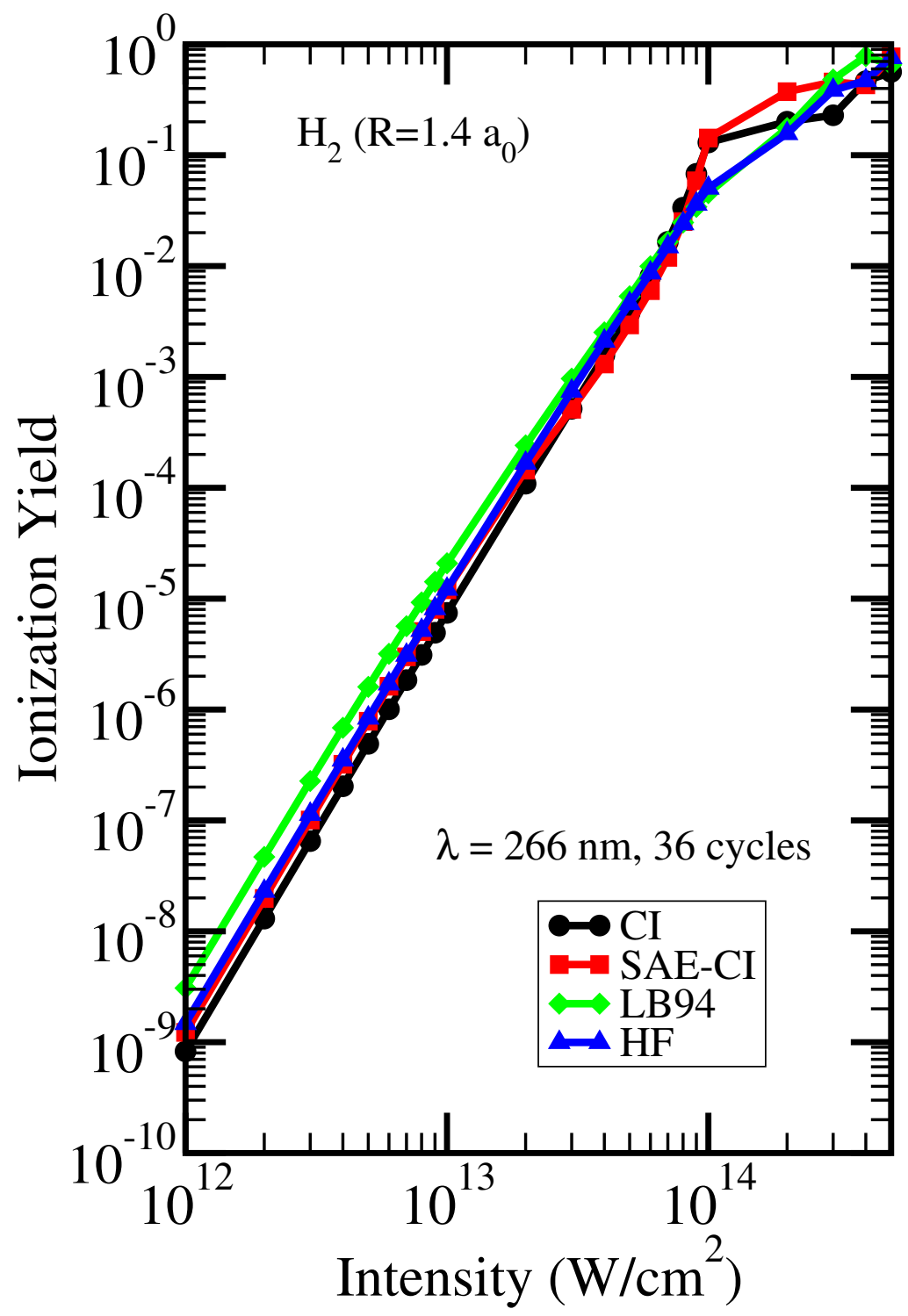

Figure 7.7: As Figure 7.5, but for $266 \mathrm{~nm}(4.65 \mathrm{eV})$ and 36 cycles (about $32 \mathrm{fs}$ ).

For a wavelength of $266 \mathrm{~nm}$ (third harmonic of $800 \mathrm{~nm}$ ) the overall agreement between the ionization yields predicted by the SAE models and full CI is good, as can be seen in Figure 7.7. In this case also the SAE-CI works very well, in fact it yields the best results. HF and LB94 agree very well with each other above about $4 \cdot 10^{13} \mathrm{~W} / \mathrm{cm}^{2}$. Below 
this intensity the LB94 yield is larger than the one obtained with HF. Since already HF overestimates the yield slightly, LB94 deviates from the CI result even more than HF for these intensities. Above $10^{14} \mathrm{~W} / \mathrm{cm}^{2}$ the agreement of $\mathrm{HF}$ and LB94 with the full CI calculation is not so good, since the CI yield shows some structure that is absent in HF and LB94, but also present in the SAE-CI yield, though the quantitative agreement with the full CI result is not at all perfect. The overall rather satisfactory agreement between the CI and the SAE models at this rather short wavelength may appear surprising on the first glance, since one usually expects the structure to become more important as the wavelength decreases. However, it has to be reminded that for this photon energy of $4.65 \mathrm{eV}$ one finds that 4 photons are required for reaching the ionization threshold for all shown models, since the ionization potentials vary only between 15.32 (LB94) and $16.19 \mathrm{eV}(\mathrm{HF})$. Furthermore, the ponderomotive shift is inversely proportional to $\lambda^{2}$ and thus quite small for $\lambda=266 \mathrm{~nm}$ compared to $\lambda=400$ or even $800 \mathrm{~nm}$. On the other hand it is worth emphasizing that the good agreement between SAE and CI is in fact not as good, if one reminds that according to the discussion above the SAE results should be multiplied by a factor 2 in order to account for the two equivalent electrons. Since the SAE yields are already without this factor slightly larger than the CI results, a factor 2 will increase the difference.

The importance of the details of the electronic structure at this wavelength becomes on the other hand clearly evident, if the excitation yield shown in Figure 7.8 is considered. The different models predict very different excitation yields that can vary by many orders of magnitude. Also the intensity dependence and thus the slope of the curves differs dramatically between the different models. The best overall agreement with the CI result is found for HF. However, for intensities above $8 \cdot 10^{13} \mathrm{~W} / \mathrm{cm}^{2}$ the agreement between $\mathrm{CI}$ and SAE-CI is very good and much better than any agreement found between CI and the two other SAE models. It is interesting to note that, for example, at $10^{13} \mathrm{~W} / \mathrm{cm}^{2} \mathrm{SAE}-\mathrm{CI}$ predicts a larger excitation than ionization yield, while the full CI calculation gives an excitation yield that is orders of magnitude smaller than the one of ionization. Nevertheless, the absolute ionization yield predicted by the two models agrees very well at this intensity (Figure 7.7). As was discussed in Section 7.1, for short wavelengths the results of different models can differ dramatically due to the difference in excitation energy and thus position of intermediate resonances. The largely overestimated excitation yield of LB94 at $266 \mathrm{~nm}$ is due to the fact that within this model the B' state is in three-photon resonance. This is immediately apparent, if the excitation yield excluding the population of the B' state is plotted. The excitation yield drops by orders of magnitude and agrees at low intensities well with the HF and the CI predictions. The overestimation of the ion yield found for the LB94 model compared to $\mathrm{HF}$ is also a consequence of this REMPI process. Since the relative importance of REMPI, i.e. the enhancement of the ionization yield due to intermediate resonances, decreases with increasing intensity the better agreement of the HF and LB94 ionization yields for high intensities is understandable. 


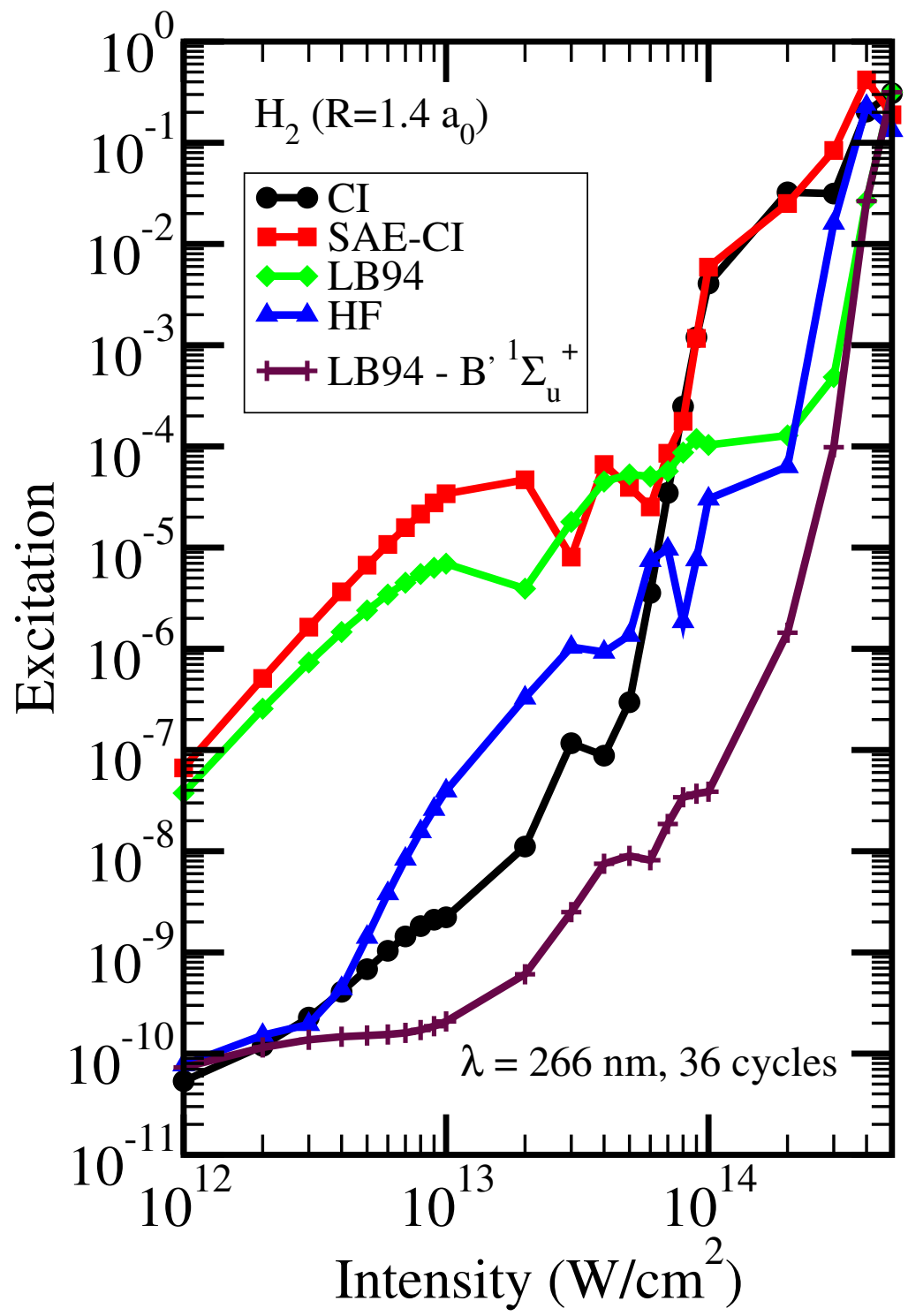

Figure 7.8: As Figure 7.7, but showing the excitation yield. The additional curve (maroon crosses) is the excitation yield obtained with the LB94 functional, but omitting the contribution of the at this wavelength resonant $\mathrm{B}^{1}{ }^{1} \Sigma_{u}^{+}$state.

\subsection{Comparison to MO-ADK}

MO-ADK is an extension of the atomic ADK model to molecules(see Section 4.2). In simple terms, this extension is achieved by fitting the long-range behavior of the molecular orbital asymptotically to hydrogen-like orbitals. Since for the latter the ADK formula was derived, it is possible to obtain MO-ADK rates from these fit coefficients, the socalled $C_{l, m}$. In the present case $m=0$ and the $C_{l} \equiv C_{l, m=0}$ are listed in Table 3.1. 
There are two practical problems related with the $C_{l}$ coefficients. First, the asymptotic form to which the molecular orbital is fitted is only approximate. Second, the obtained coefficients depend quite strongly on the model and the numerical quality of the electronic-structure calculation with which the molecular orbitals are obtained, as is discussed in [109]. In [109] it was also found that this difference is even more pronounced for larger molecules than $\mathrm{H}_{2}$. Nevertheless, as is evident from Table 3.1, even for $\mathrm{H}_{2}$ rather substantial differences between the $C_{l}$ coefficients can be found. While the HF coefficients of this work compare reasonably well with the ones obtained from a numerical HF code used in [109], the LB94 coefficients differ by about a factor of 2 . Note that in the present approach the long-range asymptotic tail of the HOMO should be accurately described by the long-range B-spline expansion, so that the $C_{l}$ coefficients extracted are expected to be numerically accurate.

The ionization yields obtained within the MO-ADK model by an integration of the rates over the corresponding laser pulses are shown for a laser wavelength of $800 \mathrm{~nm}$ in Figure 7.9. Despite the very different ionization potentials and $C_{l}$ coefficients there is rather good agreement between the HF and the LB94 MO-ADK ionization yields. Clearly, the difference in ionization potential is largely compensated by the $C_{l}$ coefficients. However, the slope obtained for HF is slightly larger than the one of LB94. On the other hand, the use of the TSLDA functional for calculating the MO-ADK yield results in a much smaller value, in agreement with the higher ionization potential and thus not compensated by the $C_{l}$ coefficients. In fact, the deviation between the MO-ADK yields obtained with LB94 or TSLDA is very similar to the one for the corresponding SAE-TDSE calculations (Figure 7.3).

In Figure 7.9 the predictions of the different MO-ADK models are also compared to the SAE-TDSE (LB94) and the full CI-TDSE results. (In view of the similarity of the SAE-TDSE ionization yields using either LB94 or HF (Figure 7.3) only the LB94 result is shown.) In the most favorable case, MO-ADK (LB94) should agree with SAETDSE (LB94). Every deviation is a clear indication of a failure of the MO-ADK model itself, since both calculations are based on the SAE and an identical description of the molecular structure. Clearly, MO-ADK predicts a wrong overall intensity dependence of the ion yield. Since the slope of the MO-ADK curve is too large but crosses the SAETDSE yield at a peak intensity of about $6 \cdot 10^{13} \mathrm{~W} / \mathrm{cm}^{2}$, the predicted ion yield is too small at lower intensities and reaches too early saturation, i. e. complete single ionization. Only for intensities between $8 \cdot 10^{13}$ and $1 \cdot 10^{14} \mathrm{~W} / \mathrm{cm}^{2}$ MO-ADK predicts the intensity dependence of the ionization yield in a qualitatively correct way, but overestimates it by about a factor of 2. This leads to an accidental agreement to the CI-TDSE result.

An analysis of the Keldysh parameter $\gamma_{\text {Kel }}$ (see Equation 1.2) reveals that MO-ADK is in fact not really expected to work well even for $800 \mathrm{~nm}$ radiation. A condition for the applicability of a tunneling model is that the Keldysh parameter should be much smaller than 1. Within HF or LB94 and at $800 \mathrm{~nm} \gamma_{\mathrm{Kel}}$ is close to 1 at about $1 \cdot 10^{14} \mathrm{~W} / \mathrm{cm}^{2}$ which sets a lower limit to the applicability of MO-ADK. On the other hand, for intensities above about $2.5 \cdot 10^{14} \mathrm{~W} / \mathrm{cm}^{2}$ one reaches the classical over-the-barrier regime, i. e. the electron can escape over the field-suppressed potential barrier and ADK is known to overestimate the ionization rates. At this intensity $\gamma_{\mathrm{Kel}}$ has, however, only dropped to 


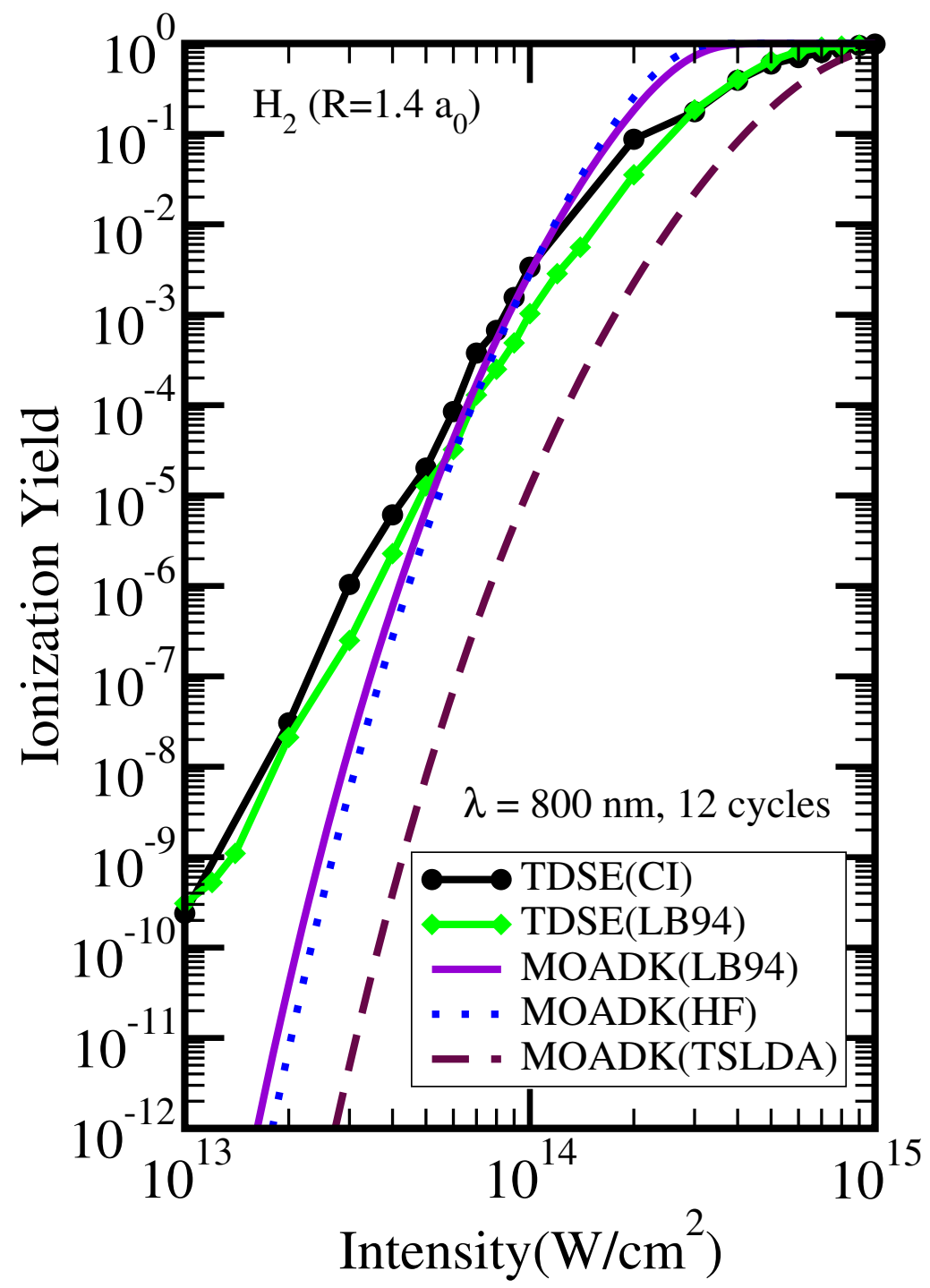

Figure 7.9: Ionization yield of $\mathrm{H}_{2}$ for the same laser parameters as in Figure 7.3. The results of CI-TDSE (black circles) and LB94 SAE-TDSE (green diamonds) are compared to the MO-ADK prediction using the molecular parameters obtained with LB94 (purple solid), HF (blue dotted), and TSLDA (maroon dashes).

about 0.72 . In the present example $\left(\mathrm{H}_{2}, 800 \mathrm{~nm}\right)$ qualitative agreement of MO-ADK with SAE-TDSE is found for $\gamma_{\mathrm{Kel}}$ close or slightly larger than 1 . For $\gamma_{\mathrm{Kel}}$ slightly smaller than 1 where ADK should be more appropriate the agreement is, however, worse. Therefore, the found small range of qualitative agreement is shifted to slightly lower intensities than expected on the basis of the Keldysh argument.

In view of the fact that MO-ADK is expected to be valid only in a small intensity 
window for a laser wavelength of $800 \mathrm{~nm}$, the value of the comparison shown in Figure 7.9 may appear questionable, at least on a first glance. However, the validity criterion is neither strict nor does it provide any information about the extent of a possible failure. The present work shows how MO-ADK fails both qualitatively and quantitatively for $\mathrm{H}_{2}$ and the popular Ti:Sapphire laser wavelength of $800 \mathrm{~nm}$. Furthermore, the ADK model has often been used outside its range of validity to explain atomic or molecular behavior in intense laser fields. One example is the already mentioned suppressed ionization in molecules [146-149]. Noteworthy, MO-ADK was used to predict the occurrence or absence of suppressed ionization for a number of molecules in a correct way [103, 109], although the adopted parameters would formally not allow to apply ADK theory, since the Keldysh parameter was too large or the over-the-barrier ionization regime was reached. Another example is the observed lower saturation intensity of different charge states of fullerenes compared to the ADK prediction and its attribution to a failure of the SAE in [130]. However, such an interpretation implicitly assumes ADK to correctly predict SAE yields.

In conclusion, Figure 7.9 indicates that for $\mathrm{H}_{2}$ and $800 \mathrm{~nm}$ MO-ADK fails even qualitatively to predict the overall intensity dependence of the ionization yield. Therefore, MO-ADK can give in the best case only partially qualitatively correct predictions, while quantitative agreement occurs only accidentally. This is in agreement with an earlier work in which it was found that MO-ADK predicts the $R$ dependence of the ionization rate of $\mathrm{H}_{2}$ at $800 \mathrm{~nm}$ qualitatively correctly in the intensity range $10^{13}$ to $10^{14} \mathrm{~W} / \mathrm{cm}^{2}$, but its quantitative prediction is wrong (see Subsection 6.2.1). As is clear from Figure 7.9, the quantitative agreement at $10^{14} \mathrm{~W} / \mathrm{cm}^{2}$ found in Subsection 6.2.1 is accidental, since it is due to a crossing and ignores the factor 2 for the two equivalent electrons. Therefore, claims based on quantitative arguments like, e. g., on a deviation between saturation intensities predicted by ADK and experimentally observed ones are problematic. 



\section{First excited state}

The development of ultrashort intense laser pulses has opened up many avenues in the area of strong field physics. These pulses could provide a tool for the time-resolved imaging of the electronic and nuclear structure of molecules. It was shown that nuclear dynamics can be resolved on the sub-Ångstrom and sub-femtosecond level [168, 169, 175, 176]. In a first step towards time-resolved imaging of electronic-structure changes, it was demonstrated experimentally that the molecular response to intense laser fields allows the retrieval of electronic-structure information. This was done based on the analysis of either the emitted high-harmonic radiation [2] or electrons [177]. Even the orientationdependent ionization probability either obtained with pre-oriented molecules or using electron-ion coincidence techniques may bear signatures of the electronic structure [23, 178-180]. A common feature of these imaging techniques is the choice of the experimental parameters in such a way that the laser-molecule interaction is supposed to take place in the so-called tunneling regime (see Figure 1.1).

Most of the more detailed studies of the behaviour of neutral molecules exposed to intense laser pulses have, however, concentrated on molecules like $\mathrm{H}_{2}, \mathrm{~N}_{2}$, or $\mathrm{O}_{2}$ with relatively large ionization potentials and a quite large gap between the electronic ground and first-excited state (of same spin symmetry). In order to assess the generality of imaging schemes based conceptually on tunneling ionization, it is of interest to consider the accessibility of the tunneling regime for molecules with a smaller gap between the ground and excited states. In order to simulate a small gap to excited states, one may consider $\mathrm{H}_{2}$ in its first excited $\mathrm{B}^{1} \Sigma_{u}^{+}$state. $\mathrm{H}_{2}$ molecule is chosen for this purpose because CI-TDSE method can be used for exact non-perturbative treatment of the $\mathrm{H}_{2}$ molecule.

In fact, the behaviour of electronically excited atoms or molecules in intense laser fields is of interest by itself. For example, the laser-induced creation of electronically excited states has been identified as a possible source for non-sequential double-ionization [21]. The knowledge about the ionization probability of such excited states in laser fields is then an evident prerequisite for the theoretical modeling of non-sequential double ionization. The previous results on $\mathrm{H}_{2}$ molecule show that a non-negligible fraction of $\mathrm{H}_{2}$ molecules is left in an electronically excited state after exposing ground-state molecules even to non-resonant laser pulses. Very recently, the neutral helium atoms left in an electronically excited state after exposing helium to laser pulses were also directly measured [165]. In view of the rapid development of the field, it does not seem unrealistic that in the near future direct measurements on excited atoms and molecules exposed to intense laser fields may become possible. Finally, such excited states may play an important role as transient intermediates in photoreactions. Again, it is important to understand whether the proposed time-resolved imaging schemes based on tunneling concepts are applicable in this case. Clearly, the theoretical treatment of 
electronically excited states in intense laser fields is challenging, since the most popular molecular strong-field ionization models, the molecular Ammosov-Delone-Krainov (MOADK) model [103] and the molecular strong-field approximation (MO-SFA) [108, 153, $157,181]$ only consider the initial, but completely ignores excited states.

Nevertheless, based on simplified models, strong-field ionization of the $\mathrm{B}^{1} \Sigma_{u}^{+}$state of $\mathrm{H}_{2}$ has been subject of previous studies. In [164] its ionization rate has been calculated in a strong static electric field and thus within the quasi-static approximation for a laser field. It was found that the ionization rate is larger than the one of the higher lying $\mathrm{E}, \mathrm{F}^{1} \Sigma_{g}^{+}$state. This is in contrast to the predictions of tunneling models in which the ionization rate depends mainly on the ionization potential. In the opposite limit of perturbative one-photon ionization, it was very recently suggested that the $\mathrm{B}^{1} \Sigma_{u}^{+}$state fulfills all criteria for the applicability of a newly developed single-active-electron approximation based on Koopmans' picture [119].

The aim of this Chapter is to study the first excited state $\mathrm{B}^{1} \Sigma_{u}^{+}$of $\mathrm{H}_{2}$ when exposed to intense laser fields and test the validity of SAE approximation for the excited state. The $\mathrm{H}_{2}$ molecule is treated in the fixed nuclei approximation at $R=2.2998 a_{0}$ with the field polarization being parallel to the internuclear axis. The $\mathrm{E}, \mathrm{F}^{1} \Sigma_{g}^{+}$state at $R=2.2998 a_{0}$ will be studied over a large range of laser parameters. Starting with $2000 \mathrm{~nm}$ where tunnel ionization is expected. Then going on to $800 \mathrm{~nm}$ and $400 \mathrm{~nm}$ which is standard Ti:Sa laser and its second harmonic, respectively. Single photon ionization over a range of photon energies will be studied with special emphasis on $8 \mathrm{eV}$ photon energy for which the results were published [119]. For checking the validity of quasi-static approximation for $\mathrm{E}, \mathrm{F}^{1} \Sigma_{g}^{+}$and $\mathrm{B}^{1} \Sigma_{u}^{+}$states [164] also the results for $R=1.4 a_{0}$ at $3000 \mathrm{~nm}$ will be shown. Since $\mathrm{E}, \mathrm{F}^{1} \Sigma_{g}^{+}$state is energetically higher than the $\mathrm{B}^{1} \Sigma_{u}^{+}$state, a further increase in wavelength is needed to fulfill the conditions of quasi-static approximation for both the states.

Ionization studies of $\mathrm{H}_{2}$ using excited states have shown that ionic states $\mathrm{H}^{+} \mathrm{H}^{-}$are very important for the ionization process [86, 114, 182]. The ionic states provide the doorway for ionization. This ionic character of the states is can describe enhanced ionization at $R \approx 6$ a.u. Thus, in any description of excited states the ionic states must be carefully included. Within the SAE approximation, the description of ionic character is not easy. So it will interesting to see this aspect of the SAE-models.

\begin{tabular}{|l|l|l|l|l|}
\hline Approach & $\mathbf{H}_{2} \mathbf{X}^{1} \Sigma_{g}^{+}$ & $\mathbf{H}_{2} \mathbf{B}^{1} \Sigma_{u}^{+}$ & $\mathbf{H}_{2}{ }^{+} \mathbf{X}^{2} \Sigma_{g}$ & $\mathbf{I}_{\mathbf{p}}\left(\mathbf{B}^{1} \Sigma_{u}^{+}\right)$ \\
\hline K-SAE & n.a. & -0.7479 & -0.5988 & 0.1490 \\
CI & -1.10374 & -0.7485 & -0.5989 & 0.1496 \\
p-SAE & -1.06385 & -0.7388 & -0.5989 & 0.1399 \\
DFT-SAE & -1.08196 & -0.8381 & -0.5989 & 0.2392 \\
\hline
\end{tabular}

Table 8.1: Energies of states in $\mathrm{E}_{h}$ using different approaches at $R=2.2998 \mathrm{a}_{0}$. Ionization potential from the $\mathrm{B}^{1} \Sigma_{u}^{+}$states is shown in the last column. The K-SAE values have been taken from [119]. 
In Table 8.1 the energies of ground $\left(\mathrm{X}^{1} \Sigma_{g}^{+}\right)$and first excited $\left(\mathrm{B}^{1} \Sigma_{u}^{+}\right)$state of $\mathrm{H}_{2}$ at $R=2.2998 \mathrm{a}_{0}$ are shown. The ground state energy of the $\mathrm{H}_{2}^{+}$ion is shown along with the ionization potential for the $\mathrm{B}^{1} \Sigma_{u}^{+}$state. The energies obtained by using the timedependent extension of Koopmans' picture with SAE approximation (K-SAE), as used in [119], CI , p-SAE and DFT-SAE approaches are shown. The energy values predicted by K-SAE and CI are in good agreement. The energies from the p-SAE approach are slightly off, while the energies from DFT-SAE do not show agreement except for the ground state. The difference of approximately $2.7 \mathrm{eV}$ in the ionization potential from the $\mathrm{B}^{1} \Sigma_{u}^{+}$state is very large. The reason for the disagreement could be that DFT is applicable only to the ground state and specific treatment of excited states is not possible within this framework of DFT.

\section{$8.12000 \mathrm{~nm}$}

The ionization potential for the $\mathrm{B}^{1} \Sigma_{u}^{+}$state in CI approach is 0.14952 a.u. ( $\left.4.0686 \mathrm{eV}\right)$. In order to have something close to tunnel/field ionization where a large number of photons are needed the wavelength has to be considerably large. At the wavelength of $2000 \mathrm{~nm}(0.6199 \mathrm{eV})$ seven photons will be needed for ionization. In Figure 8.1 ionization, population of other bound states (OBS, all bound states except the initial $\mathrm{B}^{1} \Sigma_{u}^{+}$state) and population of the $2^{1} \Sigma_{u}\left(B^{\prime}{ }^{1} \Sigma_{u}\right)$ state are shown. The pulse length is 6 cycles which is approximately $40 \mathrm{fs}$ and the calculation is done in velocity gauge using $\cos ^{2}$ pulse as given in Equation 2.22. Generally excitation yield (population of all bound states except the ground state) is plotted, but since the initial/starting state is already an excited state so it is better to look at the other bound states excluding the initial excited state.

Since there are many closely spaced states in between $\mathrm{B}^{1} \Sigma_{u}^{+}$state and the ionization threshold, it is likely that with a small photon energy one of these states can be resonantly excited and signatures of resonance enhanced multi-photon ionization (REMPI) may be seen. The presence of large number of excited states in a small energy interval between the $\mathrm{B}^{1} \Sigma_{u}^{+}$state and the ionization threshold makes the case quite different from the one when the starting state is the ground state. When starting with the ground state of $\mathrm{H}_{2}$, there is big energy gap between the ground state and the first excited state. Thus the ground state is not significantly influenced by the other states when exposed to laser fields. The large gap between the energies of ground state and first excited state reduces the possibility of REMPI for small photon energies $\left(\hbar \omega \ll I_{\mathrm{p}}\right)$. When starting with the $\mathrm{B}^{1} \Sigma_{u}^{+}$excited state, the next excited state $\mathrm{E}, \mathrm{F}^{1} \Sigma_{g}^{+}$is close and the field can couple these two states and interesting effects may be seen. Since the other states are also close by, the possibility of seeing REMPI at low photon energies are high.

The ionization curve in the low-intensity regime is also a straight line with a slope close to 6. If multi-photon ionization (LOPT) is considered then the slope should be 7. The deviation from simple multi-photon ionization picture suggests that either tunnel/field ionization or a transition from multi-photon to tunnel/field ionization regime is occurring.

The population of the OBS in the low intensity regime is mainly dominated by the 


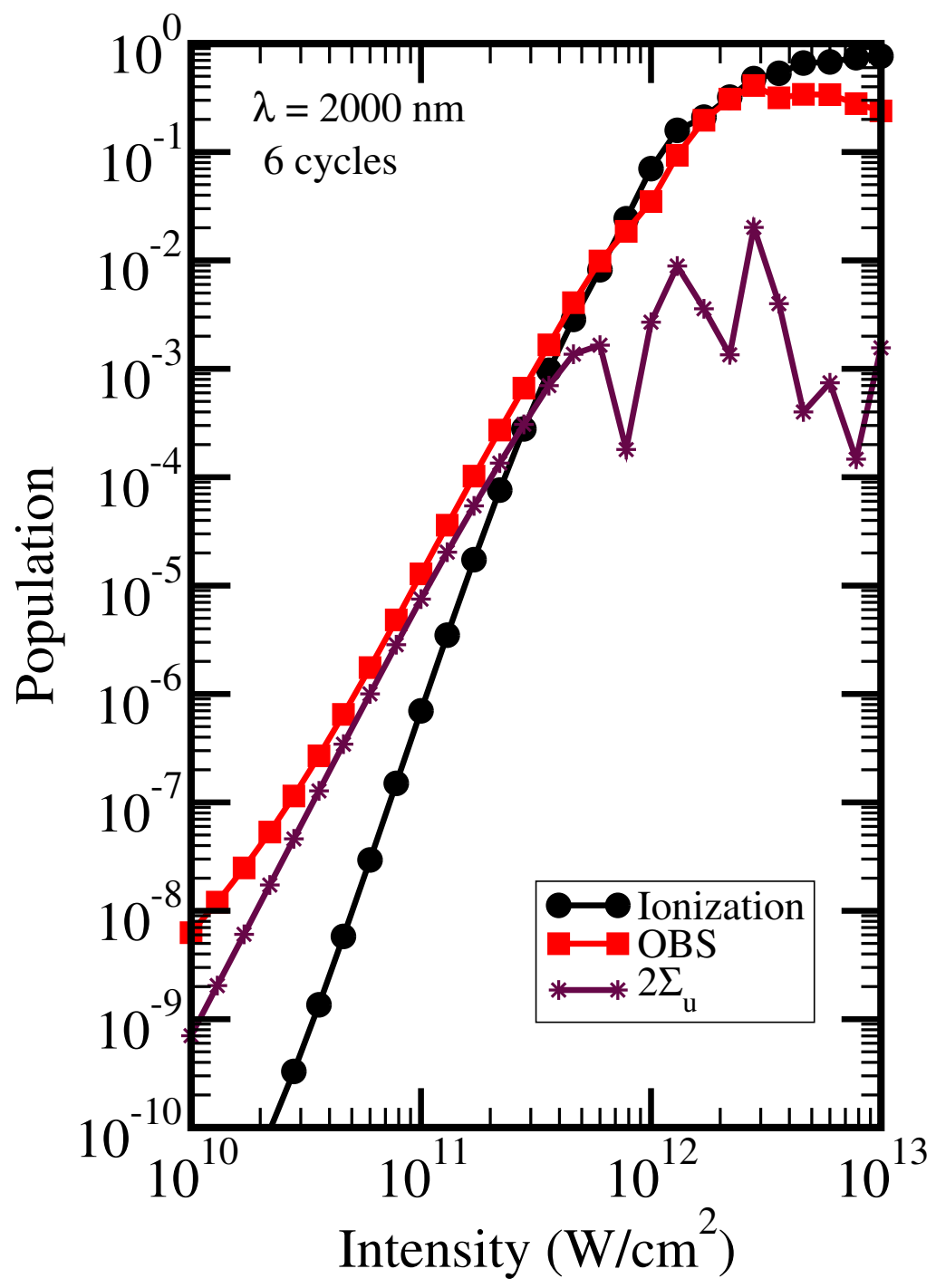

Figure 8.1: Ionization (black, circles), population of other bound states (red, squares) and population of $2^{1} \Sigma_{u}$ state (maroon, asterix) as function of peak laser intensity is shown. A $2000 \mathrm{~nm}$ laser with 6 cycles is used.

contribution from the $2^{1} \Sigma_{u}$ state. The overall behaviour of the excitation is also smooth, though it decreases slightly below the ionization curve when saturation in the ionization yield starts. Initially the population of the OBS decreases below ionization at $7.8 \times$ $10^{11} \mathrm{~W} / \mathrm{cm}^{2}$ and catches up again at $2.2 \times 10^{12} \mathrm{~W} / \mathrm{cm}^{2}$ and then finally drops down. In the region between $7.8 \times 10^{11} \mathrm{~W} / \mathrm{cm}^{2}$ and $2.2 \times 10^{12} \mathrm{~W} / \mathrm{cm}^{2}$ the ponderomotive energy $\left(U_{p}=\frac{E_{0}^{2}}{4 \omega^{2}}\right)$ varies from 0.291 to $0.822 \mathrm{eV}$. This range of ponderomotive energies contains the incident photon energy of $0.6199 \mathrm{eV}$. Thus a change in the ponderomotive energy causes the interplay between ionization and population of OBS. 
The slope of the $2^{1} \Sigma_{u}$ state in the low intensity regime on a log-log scale is close to 4 , suggesting a 4 photon excitation process. The energy difference between $\mathrm{B}^{1} \Sigma_{u}^{+}$and $2^{1} \Sigma_{u}$ states is $2.36 \mathrm{eV}$ while the energy of four $2000 \mathrm{~nm}$ photons is $2.48 \mathrm{eV}$. If broadening due to finite pulse length and ponderomotive shifts are taken into account that could lead to a resonant situation $(4+2$ REMPI). When the intensity becomes higher than $7.8 \times$ $10^{11} \mathrm{~W} / \mathrm{cm}^{2}$ the ponderomotive shift becomes significantly large, the initially resonant $2^{1} \Sigma_{u}$ state is no more resonant and leads to a chaotic behaviour in the population of the $2^{1} \Sigma_{u}$ state.

\section{$8.2800 \mathrm{~nm}$}

In Figure 8.2 ionization, population of other bound states (OBS), population of the ground state $\mathrm{X}^{1} \Sigma_{g}^{+}$and population of the $4^{1} \Sigma_{u}$ state as a function of the peak laser intensity is shown. A $800 \mathrm{~nm}(1.55 \mathrm{eV})$ laser with 6 cycles $(16 \mathrm{fs})$ is used.

The slope of the ionization curve on a log-log scale is 3, suggesting a 3 photon multiphoton process (see Equation 4.11). The ionization curve on log-log scale is a straight line for most of intensities. It only deviates from the straight line behaviour when saturation starts to set in.

The population of the other bound states (OBS) also resembles a straight line and is orders of magnitude higher than the ionization at most intensities. The population of the OBS only drops down when saturation sets in. The main contribution to the population of the OBS comes from the $4^{1} \Sigma_{u}$ state. The energy of the $4^{1} \Sigma_{u}$ state is -0.06305 a.u. $(-17.156 \mathrm{eV})$. The energy difference between the $\mathrm{B}^{1} \Sigma_{u}^{+}$and the $4^{1} \Sigma_{u}$ state is $3.21 \mathrm{eV}$ and the energy of two $1.55 \mathrm{eV}$ photons is $3.1 \mathrm{eV}$. Including the shifts in energy due to ponderomotive energy and finite width of the pulse would make these two states resonant. The log-log slope of $4^{1} \Sigma_{u}$ state is 2 . This suggests a $(2+1)$ REMPI process. The population of the ground state, $\mathrm{X}^{1} \Sigma_{g}^{+}$is orders of magnitude smaller than ionization or excitation.

\section{$8.3400 \mathrm{~nm}$}

In Figure 8.3 is similar to Figure 8.2 except that a $400 \mathrm{~nm}$ laser with 12 cycles used. The population of the resonant $5^{1} \Sigma_{g}$ is also shown. The slope of ionization curve of log-log scale is 2 , suggesting a 2 photon multi-photon process.

The population of OBS is dominated singly by the population of $5^{1} \Sigma_{g}$ state. The $\log$-log slope of $5^{1} \Sigma_{g}$ state is 1 . This suggests a $(1+1)$ REMPI process. The energy of $5^{1} \Sigma_{g}$ state is $-0.6132 \mathrm{a}$.u. $(-17.191 \mathrm{eV})$. The energy difference between $\mathrm{B}^{1} \Sigma_{u}^{+}$and $5^{1} \Sigma_{g}$ state is $3.18 \mathrm{eV}$. A single photon of $400 \mathrm{~nm}$ wavelength has an energy of $3.1 \mathrm{eV}$. What is surprising here is that resonance is very strong and the population of $5^{1} \Sigma_{g}$ state saturates even before ionization. Even at $1.0 \times 10^{13} \mathrm{~W} / \mathrm{cm}^{2}$ the population of $5^{1} \Sigma_{g}$ state is 10 times more than the ionization.

The population of the ground state, $\mathrm{X}^{1} \Sigma_{g}^{+}$is orders of magnitude smaller than ionization or excitation. 


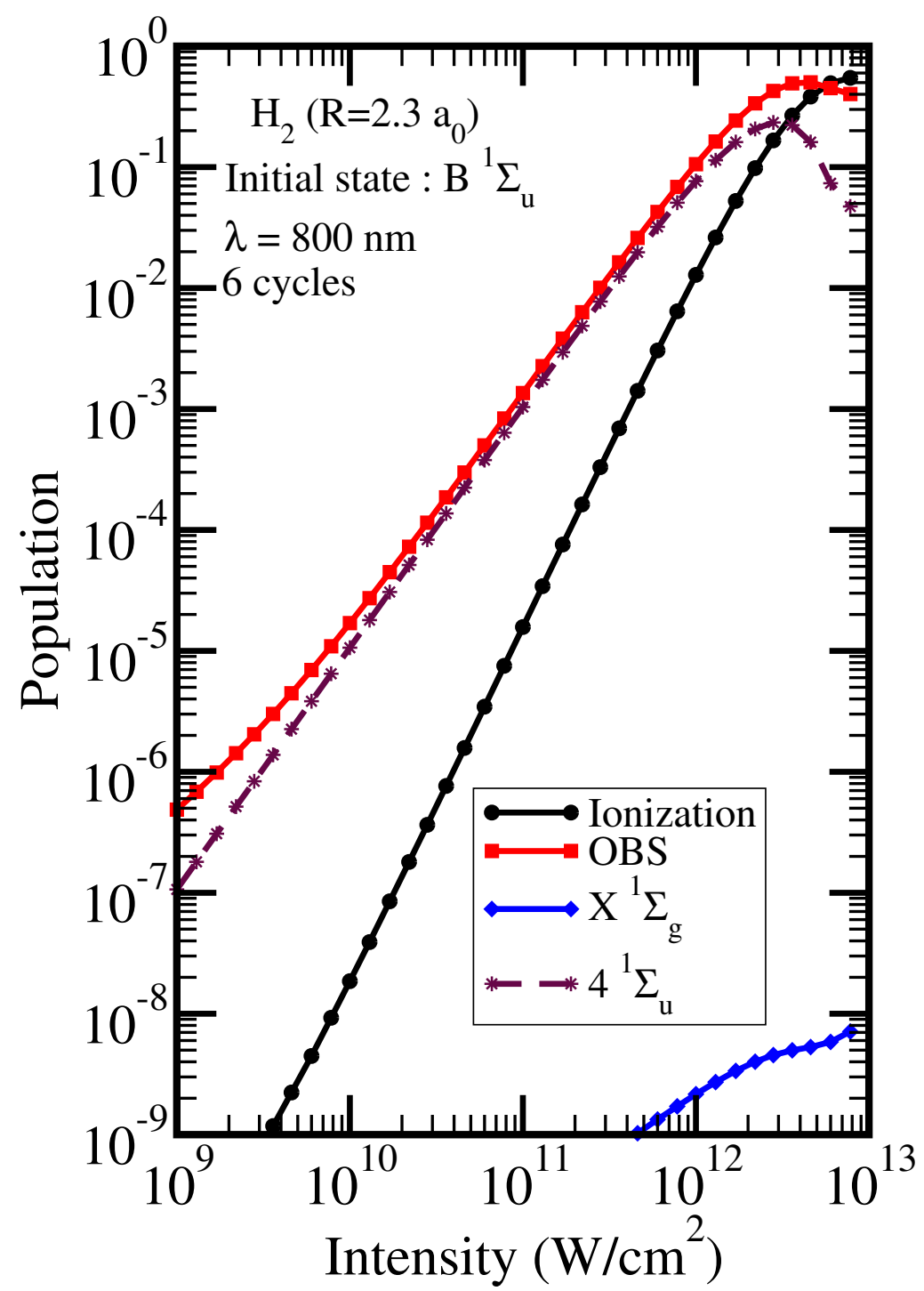

Figure 8.2: Ionization (black, circles), population of other bound states (OBS) (red, squares) and population of ground state, $\mathrm{X}^{1} \Sigma_{g}^{+}$, (blue, diamonds) and population of the $4^{1} \Sigma_{u}$ state (maroon, asterix) as function of peak laser intensity is shown. A $800 \mathrm{~nm}$ laser with 6 cycles is used.

\subsection{Single photon ionization}

In Figure 8.4 ionization yield and ground state population as a function of the incident photon energy is shown. The ionization yield is obtained from the solution of the TDSE (CI and DFT-SAE approaches) and using LOPT. The ground state population is obtained from the solution of TDSE. A $10 \mathrm{fs}$ laser pulse with a peak intensity of $1.0 \times 10^{9} \mathrm{~W} / \mathrm{cm}^{2}$ is used. The photon energies chosen cover the range of single-photon 


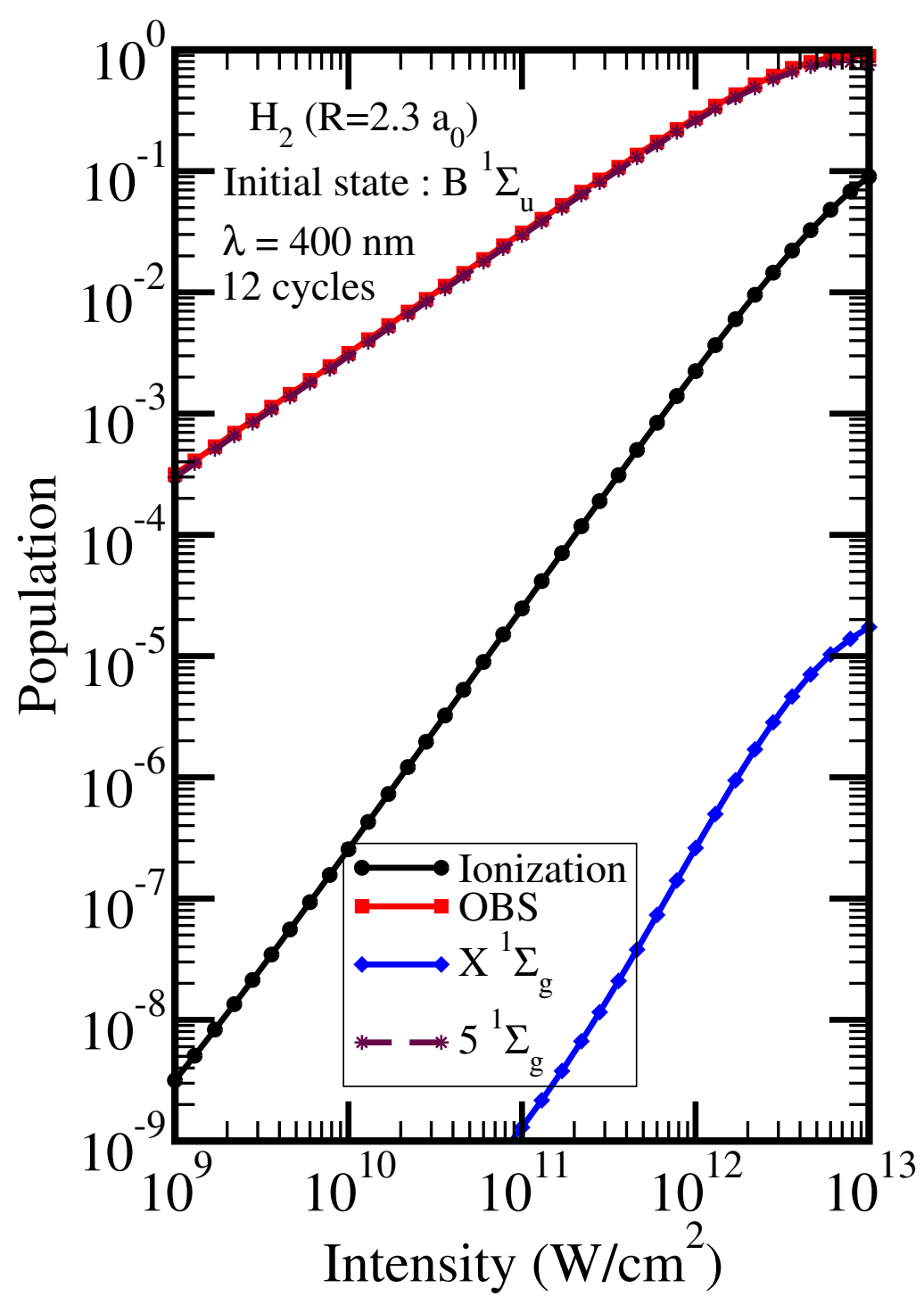

Figure 8.3: Ionization (black, circles), population of OBS(red, squares), population of ground state, $\mathrm{X}^{1} \Sigma_{g}^{+}$, (blue, diamonds) and population of the $4^{1} \Sigma_{u}$ state (maroon, asterix) as function of peak laser intensity is shown. A $400 \mathrm{~nm}$ laser with 12 cycles is used.

ionization. The upper limit of the photon energy is set by the second ionization threshold (first excited state of $\mathrm{H}_{2}^{+}$). Above the second ionization threshold the problem becomes more complex as new ionization channels start to open.

The ionization yields obtained from CI-TDSE and LOPT agree well except in the high photon energy region, where LOPT shows some peaks. These peaks are washed out in the CI-TDSE calculations but a similar trend is seen. In the TDSE there is slight increase in the ionization yield at around $11.8 \mathrm{eV}$ and a dip at around $12.0 \mathrm{eV}$. The sharp 


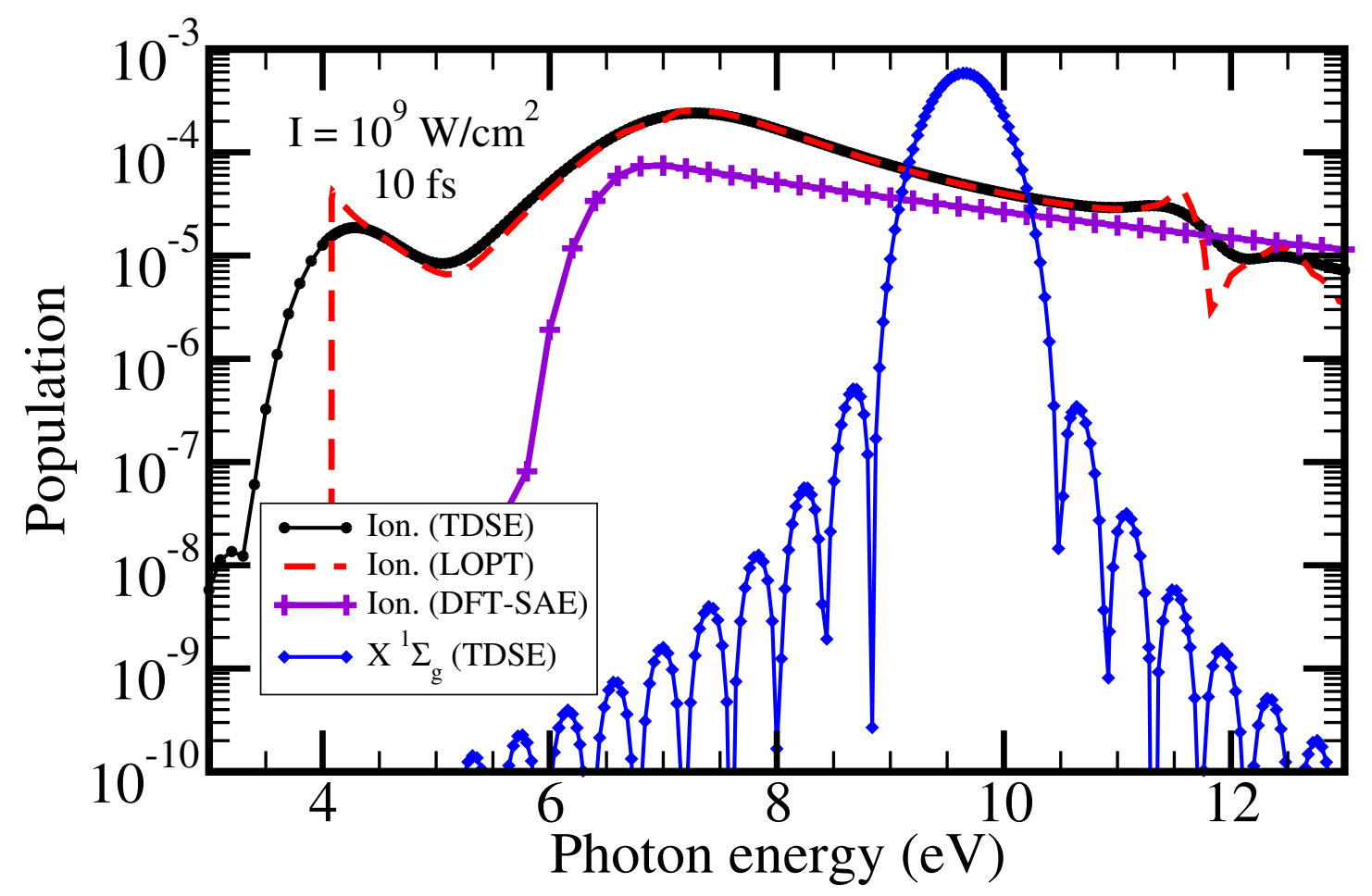

Figure 8.4: Ionization obtained with CI-TDSE (black, circles), ionization obtained with LOPT (red, dashed), ionization obtained with DFT-SAE-TDSE (violet, plus) and population of ground state, $\mathrm{X}^{1} \Sigma_{g}^{+}$, (blue, diamonds) as function of incident photon energy is shown. A 10 fs laser pulse with a peak intensity of $1.0 \times 10^{9} \mathrm{~W} / \mathrm{cm}^{2}$ is used.

peaks as seen in the result from LOPT are washed out. The ionization yield obtained from DFT-SAE-TDSE does not exactly match the CI-TDSE ionization yield, but it is quite close to the expected values. Since DFT-SAE has a different ionization potential, the drop in population at around $6.4 \mathrm{eV}$ is a result of channel closing. Above $6.4 \mathrm{eV}$ single-photon ionization takes place and below $6.4 \mathrm{eV}$ two photon ionization takes place. The DFT-SAE-TDSE fails to reproduce the structure seen at around $11.8 \mathrm{eV}$ by LOPT and CI-TDSE calculations. Even with such large difference in the ionization potential DFT-SAE-TDSE is very close to CI-TDSE.

The ground state population has a peak at $9.66 \mathrm{eV}$ which corresponds to the Rabi oscillation frequency of the ground state, $\mathrm{X}^{1} \Sigma_{g}^{+}$, and the first excited state $\mathrm{B}^{1} \Sigma_{u}^{+}$. The oscillations seen in the population of the ground state are an artifact and arise from the use of cosine squared shaped pulses. The increase in the ground state population due to Rabi oscillations is a factor 10 more than the ionization yield at $9.66 \mathrm{eV}$. Even this strong increase in the population of the ground state at $9.66 \mathrm{eV}$ has no effect on the ionization yield. The CI-TDSE ionization yield still matches the LOPT ionization yield. 


\section{$8.58 \mathrm{eV}$}

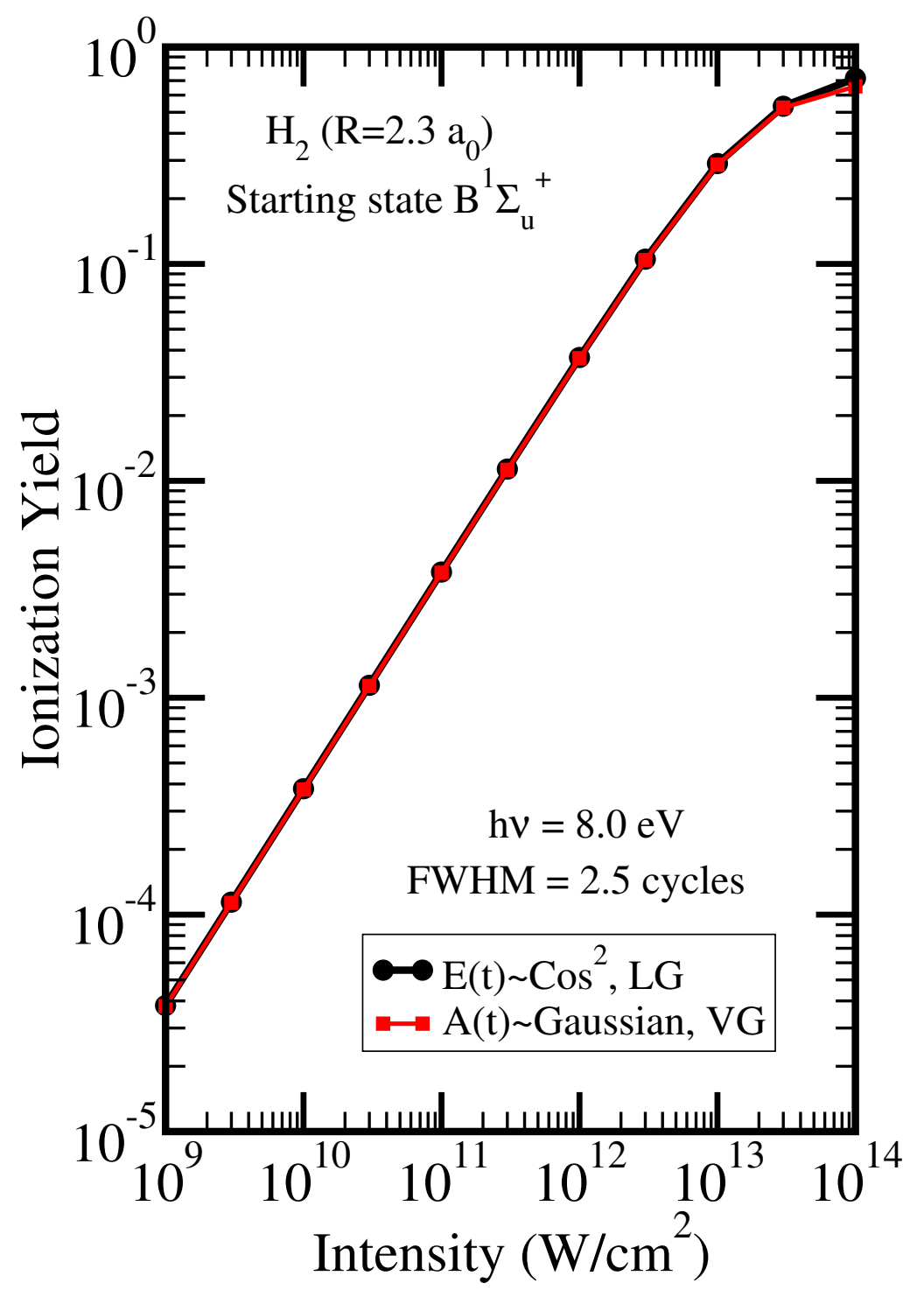

Figure 8.5: Ionization yield as function of peak laser intensity is shown. The TDSE calculation is done using length gauge transition dipole moments with a $\cos ^{2}$ envelope for the electric field (black, circles) and velocity gauge transition dipole moments with Gaussian envelope for the vector potential (red, squares). The FWHM for both the pulses is 2.5 cycles of $8 \mathrm{eV}$ photon energy.

In [119], the authors found that 5 cycles of $8 \mathrm{eV}$ photon energy laser satisfied the requirements for the fulfillment of their time-dependent extension of Koopmans picture. The calculations for two values of the electric field $\left(F_{0}=0.005\right.$ a.u. and $F_{0}=0.02$ a.u.) 


\begin{tabular}{|l|l|l|l|l|l|l|}
\hline & $\mathbf{F}_{0}$ (a.u.) & K-SAE & CI & LOPT & p-SAE & DFT-SAE \\
\hline Initial & 0.005 & n.a. & 0.960 & n.a. & 0.967 & 0.980 \\
\hline Ion. & 0.005 & 0.0043 & 0.033 & 0.037 & 0.0031 & 0.022 \\
\hline \hline Initial & 0.020 & 0.930 & 0.548 & n.a. & 0.624 & 0.728 \\
\hline Ion. & 0.020 & 0.066 & 0.379 & 0.457 & 0.036 & 0.261 \\
\hline
\end{tabular}

Table 8.2: Comparison of K-SAE, CI, LOPT, p-SAE and DFT-SAE results. The population of initial $\mathrm{B}^{1} \Sigma_{u}^{+}$state and ionization yield for two different values of electric field are shown.

were performed. The authors in [119] had used $I=\epsilon_{0} c F_{0}^{2}$ relation for the defining the peak intensity, while in this work $I=\frac{1}{2} \epsilon_{0} c F_{0}^{2}$ is used (see Equation 2.20). Even-though there is difference in the way the intensity is defined, the value of electric field $F_{0}$ is a fundamental quantity which goes in the numerical solution of the problem.

In Table 8.2 the population of the initial $\mathrm{B}^{1} \Sigma_{u}^{+}$state and the ionization yield for two values of the electric field is shown for the different approaches used, namely, K-SAE, CI, LOPT, p-SAE and DFT-SAE. The values for the K-SAE approach are taken from [119]. Within LOPT only ionization yields have been obtained. The single-photon ionization cross-section at $8 \mathrm{eV}$ is $5.75 \times 10^{-17} \mathrm{~cm}^{2}$. Using Equation 4.11 and integrating over the intensity pulse shape (Equation 5.1), the ionization yield in LOPT is calculated. The significant difference between LOPT and CI-TDSE approaches at $F_{0}=0.02$ a.u. comes from the fact that at this field strength saturation is taking place. In the saturation regime ionization calculated from LOPT continues as a straight line, while ionization calculated from TDSE undergoes a non-linear increase towards a value of unity (complete ionization). At the field strength of 0.005 the ionization yields obtained from LOPT and CI-TDSE agree well.

The p-SAE TDSE (also referred as CI-SAE before) approach does not agree with the full CI-TDSE calculation. Surprisingly, with all disagreement in the energy values, DFT-SAE-TDSE results are closer to the full CI-TDSE results. As seen from Figure 8.4, the result at $8 \mathrm{eV}$ is not a coincidence. In general, DFT-SAE is superior to K-SAE or p-SAE, at least in some range of the single photon ionization.

In Figure 8.5 the ionization yield as a function of the peak laser intensity is shown. The ionization yield obtained from using transition dipole moments calculated in length gauge and $\cos ^{2}$ variation of the electric field as given in Equation 2.22 is shown by black (circles) curve. The ionization yield obtained from using transition dipole moments calculated in velocity gauge and Gaussian variation of the electric field as given in Equation 2.23 is shown by red (squares) curve. Even using different gauges and different pulse shapes has no effect on the ionization yield, the curves are seen to overlap each other, except in the saturation regime.

In Figure 8.6 the angle averaged photoelectron spectra for carrier envelope phase (CEP) of $0^{\circ}$ and $90^{\circ}$ are shown. The laser intensity is $8.8 \times 10^{11} \mathrm{~W} / \mathrm{cm}^{2} .5$ cycles of $8 \mathrm{eV}$ 


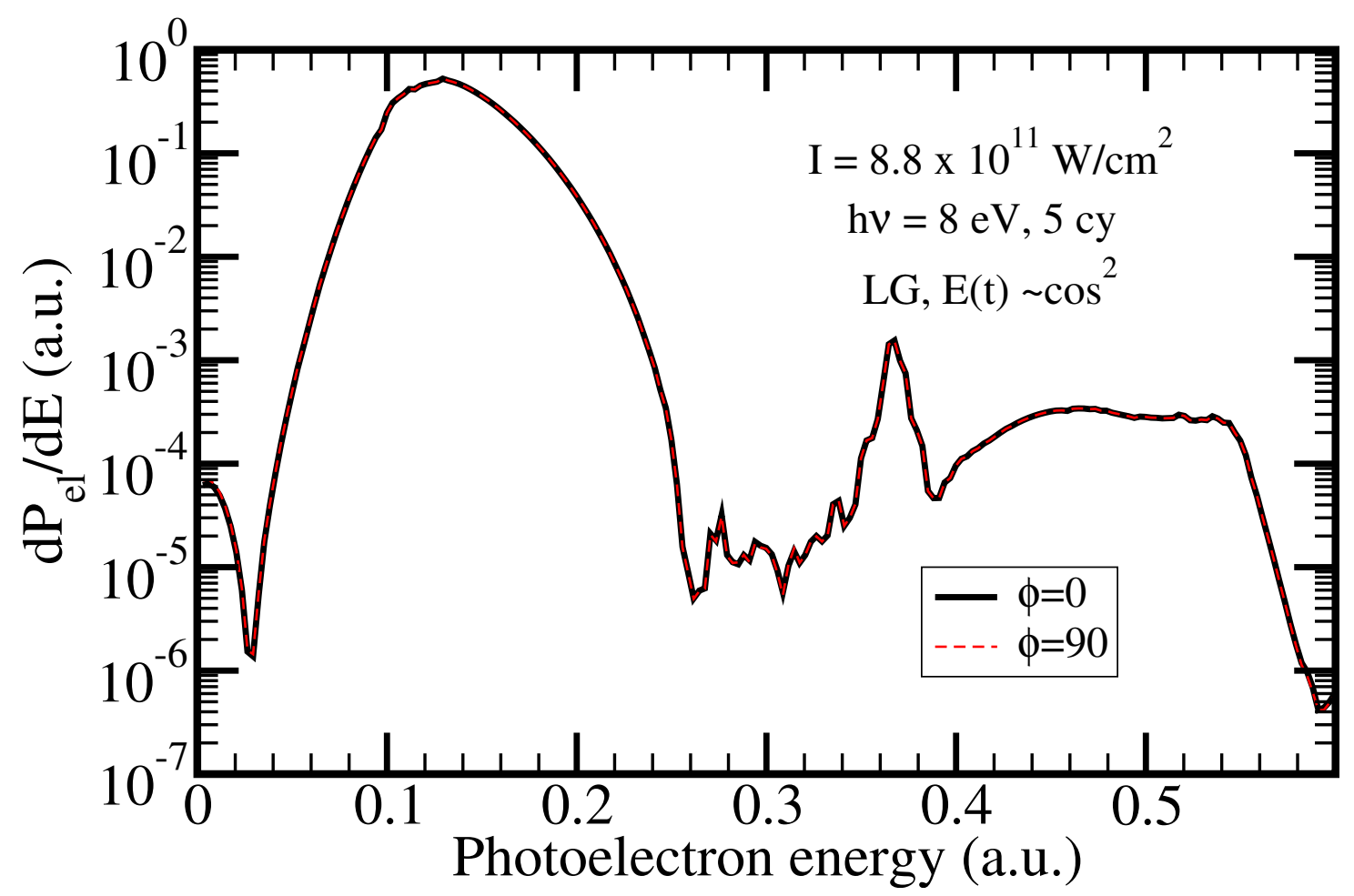

Figure 8.6: Photoelectron spectra for a peak intensity of $8.8 \times 10^{11} \mathrm{~W} / \mathrm{cm}^{2}$ using 5 cycles of $8 \mathrm{eV}$ photon energy is shown. The solid (black) curve represents a CEP of $0^{\circ}$ and dashed (red) curve represents a CEP of $90^{\circ}$.

photon energy laser is used. The calculation is done in length gauge with a $\cos ^{2}$ envelope for the electric field. It is seen that even for a 5 cycle pulse the CEP is not giving any variation in the photoelectron spectra. The curves are on top of each other for most of the energy range. It is only at large energies that a deviation is seen. Since the deviation takes place at the values which are orders of magnitude smaller, it is possible that this deviation could come from the numerical approach that has been used. The first broad peak at around 0.134 a.u. comes from single photon transition from the $\mathrm{B}^{1} \Sigma_{u}^{+}$state. The shortness of the pulse $(2.54 \mathrm{fs})$ leads to a broad peak. There is very small kink in photoelectron spectra at 0.11 a.u. which is not visible on the logarithmic scale. The inset with the linear scale shows shows the kink. The two photon ionization from the ground state causes this kink. Since the magnitude of the two photon ionization process from the ground state is small as compared to single photon ionization from the $\mathrm{B}^{1} \Sigma_{u}^{+}$state, only a kink is seen. This result is contrary to the findings of authors in [119] who observe a prominent peak for the two photon ionization from the ground state. The second peak around 0.36 a.u. comes from the three photon ionization of the $\mathrm{X}^{1} \Sigma_{g}^{+}$ground state. Again this peak is orders of magnitude smaller than the peak coming from the single photon ionization from the $\mathrm{B}^{1} \Sigma_{u}^{+}$state. These results again reflect the picture as seen in case of $800 \mathrm{~nm}$ (Section 8.2) and $400 \mathrm{~nm}$ (Section 8.3) that the contribution of ground 
state to ionization process is very small.

8.6 $R=1.4$ a.u.
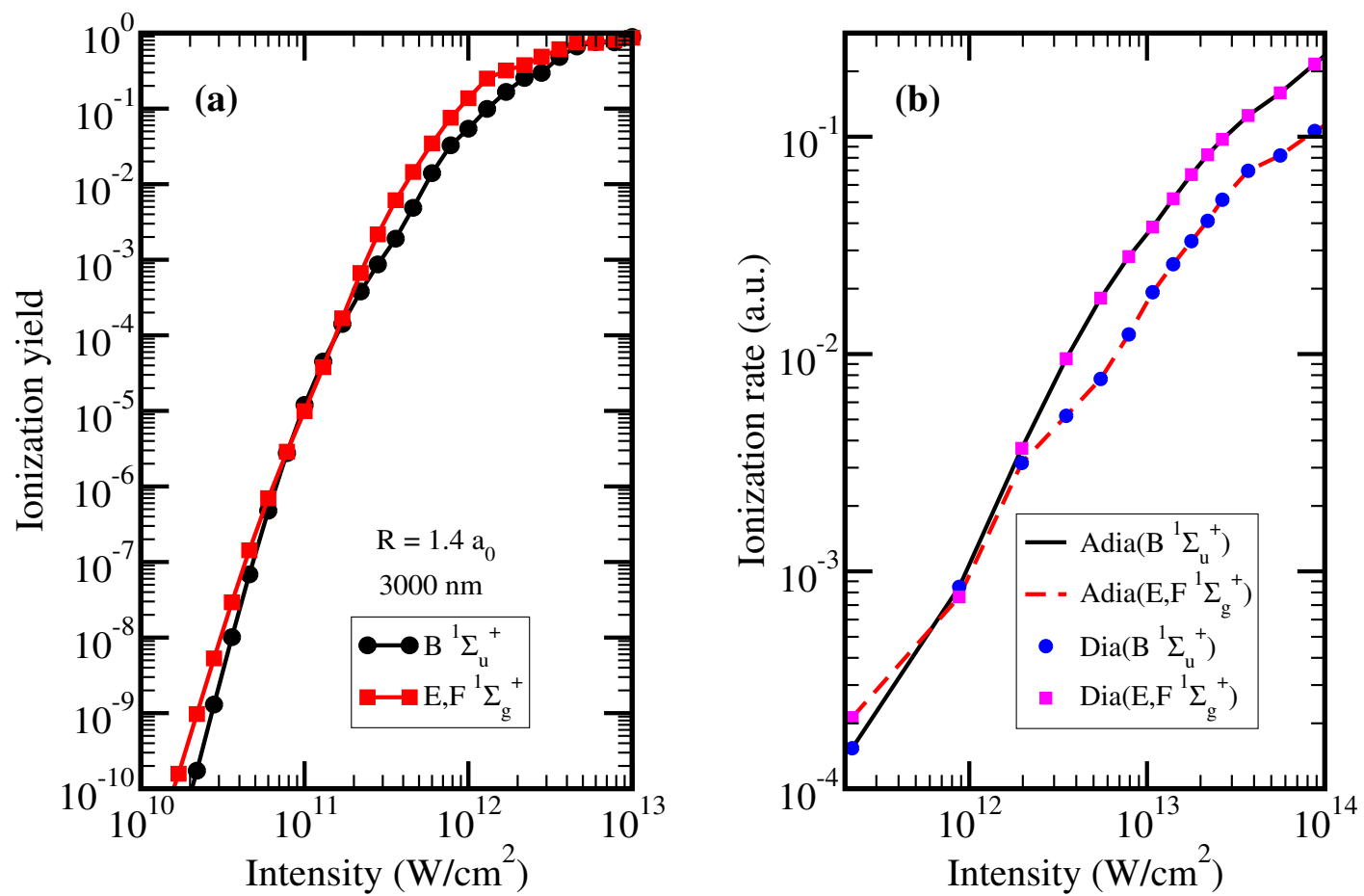

Figure 8.7: (a) Ionization as a function of peak laser intensity from $\mathrm{B}^{1} \Sigma_{u}^{+}$(black, circles) and $\mathrm{E}, \mathrm{F}^{1} \Sigma_{g}^{+}$(red, squares) states of $\mathrm{H}_{2}$ at $R=1.4 a_{0}$ is shown. (b) The adiabatic ionization rates as function of peak laser intensity (from [164]) for the two states. Also diabatic rates are shown.

There was an interesting, counterintuitive result predicted for the $\mathrm{B}^{1} \Sigma_{u}^{+}$and $\mathrm{E}, \mathrm{F}^{1} \Sigma_{g}^{+}$ excited states of $\mathrm{H}_{2}$ at $R=1.4 a_{0}$ [164]. It was predicted that within the quasi-static approximation (adiabatic limit) the ionization width of the lower lying $\mathrm{B}^{1} \Sigma_{u}^{+}$state is higher than the one of the energetically higher lying $\mathrm{E}, \mathrm{F}^{1} \Sigma_{g}^{+}$state. This result is highly counterintuitive to theories like ADK where the ionization potential is the main factor governing the ionization process. It will be interesting to see what TDSE predicts in such a situation. In order to simulate the quasi-static regime, a wavelength of $3000 \mathrm{~nm}$ was chosen. 6 cycles ( $60 \mathrm{fs}$ ) of $\cos ^{2}$ envelope pulse for the vector potential, as in Equation 2.22, are used. In Figure 8.7 a the ionization yield as a function of the peak laser intensity for the chosen laser parameters is shown. The ionization yields when starting $\mathrm{B}^{1} \Sigma_{u}^{+}$and $\mathrm{E}, \mathrm{F}^{1} \Sigma_{g}^{+}$states as initial state are shown. 
In Figure $8.7 \mathrm{~b}$ the ionization rates for the two states in adiabatic (solid and dashed lines) and diabatic (circles and squares) limits as in [164] are shown. In [164] the ionization rate was shown as a function of field strength. In the present case the field strengths have been converted to intensity values for better comparison and visibility. In [164] the results were only obtained in the adiabatic limit. These results are converted into the diabatic limit by exchanging the curves after the (avoided) crossing. There was no avoided crossing observed in [164] when looking at the energy values of the two states exposed to dc-fields, however a crossing in the ionization widths was seen. The accuracy of these calculations at low field strengths was not very high. Another problem with these results in quasi-static approximation is that in the given range of intensity or field values, the ionization rates are very high. Any pulse which shall satisfy quasi-static approximation for these two states has to be very long (more than 1000 a.u. in time). When using such pulses very high ionization yields or saturation shall be observed.

In order to validate the results in the adiabatic approximation at low field strengths, the in-field Hamiltonian was diagonalized. The resulting states were projected back onto the field-free states and analyzed. There was an avoided crossing observed at a field strength of 0.004 a.u. $\left(5.6 \times 10^{11} \mathrm{~W} / \mathrm{cm}^{2}\right.$ in intensity). This crossing was not observed in [164]. The basis set for diagonalization of in-field Hamiltonian was obtained from CI calculation which is the same as used in time propagation. Thus, the occurrence of an avoided crossing leads to such an interesting behaviour.

The ionization yield obtained from the solution of the TDSE, as shown in Figure $8.7 \mathrm{a}$ does show a cross-over at around $10^{11} \mathrm{~W} / \mathrm{cm}^{2}$. Unlike the adiabatic case, in the timedependent case, the ionization yield at higher intensities is larger for the energetically higher $\mathrm{E}, \mathrm{F}^{1} \Sigma_{g}^{+}$state. The result at high intensities contradicts the adiabatic picture. It could well be possible that adiabatic picture is not completely valid for the laser pulses used in the TDSE. Thus one should also look at the diabatic counterpart. Now if the diabatic curves for ionization rates as shown in Figure $8.7 \mathrm{~b}$ are seen. They provide a similar picture as shown in TDSE results. The diabatic curve for the ionization rate from the $\mathrm{B}^{1} \Sigma_{u}^{+}$state (shown by blue circles) crosses the ionization rate from the $\mathrm{E}, \mathrm{F}^{1} \Sigma_{g}^{+}$ state (shown by magenta squares) just below $10^{12} \mathrm{~W} / \mathrm{cm}^{2}$. However, for the next point on the curve, the ionization rate from the higher lying $\mathrm{E}, \mathrm{F}^{1} \Sigma_{g}^{+}$state takes over. In the time-dependent case, starting with an initially fixed state, it is difficult for this initial state to adiabatically change character within the finite time of the pulse. Thus, a diabatic picture provides a much better explanation of the time-dependent calculations. It can be concluded that 6 cycles of $3000 \mathrm{~nm}$ laser are not enough to provide an adiabatic picture. 



\section{Larger molecules}

The application of DFT-SAE-TDSE is not limited to $\mathrm{H}_{2}$. Even larger molecules like $\mathrm{N}_{2}$, $\mathrm{O}_{2}$ and $\mathrm{C}_{2} \mathrm{H}_{2}$ can be treated within the framework of DFT-SAE-TDSE. These molecules belong to $\mathrm{D}_{\infty h}$ which is the same symmetry group as $\mathrm{H}_{2}$, and thus have the same selection rules for electronic states when exposed to laser fields. The DFT-SAE-TDSE can also be solved for molecules like $\mathrm{CO}$ and $\mathrm{H}_{2} \mathrm{O}$ which belong to different symmetry groups by including correct implementation of the selection rules in the time-propagation part. So DFT-SAE provides a framework for the treatment of large molecules within the singleactive-electron approximation. As already seen in Chapter 7, this method works nicely for $\mathrm{H}_{2}$.

The SAE approximation used in MO-ADK (see Section 4.2) and MO-SFA [108, 153, $157,181]$ theories has successfully explained many experimental results for larger molecules. Since no exact theories exist for these larger molecules the validity regime of SAE approximation for these molecules is not known. As seen in the case of $\mathrm{H}_{2}$ molecule the SAE approximation breaks down when the ionization yield is becomes significant (more than 10\%), similar things can be expected for these larger molecules. The SAE is likely to breakdown when the laser intensity is sufficiently high and significant amount of ionization is taking place. When the laser intensity is high, it likely that multiple ionization will take place and this can not be explained by the current implementation of DFT-SAETDSE approach. The present DFT-SAE-TDSE method for larger molecules is likely to work better than MO-ADK and MO-SFA models because the density functional theory gets better when the number of elements contributing to the density increase. Thus DFT-SAE-TDSE is likely to fare better for the systems with large number of electrons, at-least in the range where SAE approximation is valid.

There is a significant amount of experimental data present for $\mathrm{N}_{2}$ and $\mathrm{O}_{2}$. The results have often been presented in the light of suppressed ionization and compared with the ionization of atoms with approximately the same ionization potential. The typical molecule-atom pairs with the same ionization potential are $\mathrm{H}_{2}$ : $\mathrm{Ar}, \mathrm{O}_{2}$ :Xe and $\mathrm{N}_{2}$ :Ar. It was experimentally shown that the ionization in $\mathrm{O}_{2}$ is suppressed when compared to ionization from Xe $[146,147]$. In a similar result, it was shown that hydrogen molecules were difficult to ionize when compared with $\mathrm{Ar}$ atoms [183]. In contrast, $\mathrm{N}_{2}$ displayed an ionization rate quite similar to the rate of $\operatorname{Ar}[146,147]$.

What makes some, but not all, diatomic molecules harder to ionize than their companion atoms? Several attempts have been made to explain the available data, particularly for the case of $\mathrm{O}_{2}$. First, the production of a doubly excited state, via collisions of a rescattered electron and the molecular ion, which then dissociates into two neutral atoms (i.e., dissociative recombination) has been proposed as a mechanism that could suppress the production of $\mathrm{O}_{2}^{+}$. Subsequent measurements [147] show little dependence of the $\mathrm{O}_{2}$ 


\section{Larger molecules}

suppression on the ellipticity of the laser polarization, indicating that the dissociative recombination mechanism is unlikely to be the source of the anomalously low $\mathrm{O}_{2}^{+}$yield. Second, a modified tunneling model that accounts for the two-center potential of a diatomic molecule [184] provides qualitative agreement with the $\mathrm{O}_{2}$ data, but is unable to explain the magnitude of the suppression [184]. Talebpour et al. [183] also consider the orientation of the two-center potential relative to the laser polarization to explain the suppression of ionization in $\mathrm{D}_{2}$. They calculate an effective charge for the atomic tunneling theory and include an effective increase in the molecular ionization potential due to the non-negligible Franck-Condon overlap between the field-free ground state and field-free vibrationally excited states of the molecular ion. They achieve good agreement between theory and experiment provided that the correct value of the effective charge is used [183]. Third, an investigation of the influence of vibrational motion and field induced changes in bond lengths on the ionization rates of $\mathrm{H}_{2}$ and $\mathrm{O}_{2}$ also predicts reduced ionization rates relative to companion atoms of the same ionization potential, but cannot quantitatively account for the observed suppression [185].

There were two results based on very different theoretical models, which were in quantitative agreement with the $\mathrm{O}_{2}$ and $\mathrm{N}_{2}$ experimental data. Both models suggest general prescriptions for treating the complexities associated with molecular structure in a straightforward way. Guo has introduced a structure correction [186] to the ADK tunneling formula (see Section 4.1). As in the case of $\mathrm{D}_{2}$ described above, the observed suppression of $\mathrm{O}_{2}$ is reproduced, provided the correct parameters for the effective nuclear charge and effective ionization potential are chosen. In the context of this model, the observed absence of suppression in $\mathrm{N}_{2}$ [147] is explained by the uniform distribution of the outer electrons around the ionic molecular core, resulting in an atom like potential with an effective nuclear charge of 1.0.

A second model, proposed by Muth-Böhm and coworkers [108] explains the suppression of $\mathrm{O}_{2}$ as a double-slit interference effect, arising from emission of electron waves from two distinct centers in the diatomic potential. The velocity gauge implementation of intense-field many-body S-matrix theory or strong field approximation (SFA) [107] gives an interference term [108]. Using this formulation of SFA they were able to correctly predict the suppression of $\mathrm{O}_{2}$ and its absence in $\mathrm{N}_{2}$. In the low energy limit where the de Broglie wavelength of the ionizing electron is long compared to the internuclear separation, completely constructive (destructive) interference occurs if electron amplitude is emitted from the respective atomic centers perfectly in phase (out of phase). In molecules with symmetric electronic ground-state wave functions, e.g., $\mathrm{N}_{2}$, the interference is constructive and ionization via ejection of low energy electrons proceeds as if from a single atomic center. On the other hand, in molecules with antisymmetric ground states, e.g., $\mathrm{O}_{2}$, the interference is destructive, totally suppressing ionization through dominant low energy channels. Indeed, as predicted, subsequent measurements of above-threshold ionization (ATI) show an absence of low energy electron emission during strong-field ionization of $\mathrm{O}_{2}$ [187]. Muth-Böhm et al. [108] also made a quantitative prediction of ionization suppression of $\mathrm{F}_{2}$ relative to $\mathrm{N}_{2}$. In case of $\mathrm{F}_{2}$ both the models fail to explain the experimentally observed non-suppression [188]. Moreover, the interference model predicts ionization suppression in all diatomic molecules with anti- 
symmetric electronic ground states. The formalism was later generalized to symmetric polyatomic molecules as well [189]. The conclusion of [189] is that multi-centered complex molecules cannot have a higher ionization rate than single centered atoms, since the interference effect can only decrease the total probability for ionization. Indeed, suppressed ionization has been observed in $\mathrm{C}_{6} \mathrm{H}_{6}$ molecules [190, 191]. If suppression due to interference effects is a general phenomenon in molecular ionization, the model provides a useful tool for calculating accurate ionization rates of many molecules.

The suppression predicted by SFA due to interference depends on the gauge. The interference picture appears only in the velocity gauge. The scientific community is now divided into two groups, using the two gauges, i.e. length and velocity gauge. The issue of gauges has been there for a long time, starting with KFR (Keldysh-Faisal-Reiss) theory $[24,150,151]$ but no agreement has been reached so far. For the case of $\mathrm{H}_{2}$ molecule, a systematic study of various MO-SFA implementations using length and velocity gauge was performed in [172]. The SFA in length and velocity gauge was compared to a full CI calculation (CI-TDSE). The results could not establish supremacy of one gauge over the other for the different basis sets used within the SAE approximation.

At the moment, there is no agreement in the SFA community on which gauge to use. So, after the successful implementation of DFT-SAE-TDSE for $\mathrm{H}_{2}$, now the application of DFT-SAE-TDSE is extended to larger molecules. In this chapter, the results for the $\mathrm{N}_{2}, \mathrm{O}_{2}$ and $\mathrm{C}_{2} \mathrm{H}_{2}$ molecules are presented. These results have been obtained at equilibrium internuclear distance for different molecules. All the results are for parallel orientation of the laser field polarization with respect to the major molecular axis.

\subsection{Photon energy scan}

In Figure 9.1 ionization and the sum of ionization and excitation, as shown before in Figure 5.1, as a function of photon energy for $\mathrm{O}_{2}$ molecule is shown. The laser intensity is $1.0 \times 10^{12} \mathrm{~W} / \mathrm{cm}^{2}$ and 15 fs pulse with cosine squared envelope for the vector potential (Equation 2.22) is used. The ionization potential for $\mathrm{O}_{2}$ as obtained from DFT-SAE calculations is $12.49 \mathrm{eV}$. The symmetries involved in case of $\mathrm{O}_{2}$ molecule are $\Pi_{g}$ and $\Pi_{u}$ $(m=1)$. The ground state has $\Pi_{g}$ symmetry and the overall symmetry of the ground state is odd. Going by the interference model of Muth-Böhm and coworkers [108, 189], the suppressed ionization should be seen when starting with the ground state as the initial state.

In Figure 9.1 a peak in the sum curve (red, square) at $10.0 \mathrm{eV}$ suggests that excitation is very high at this photon energy. The increase in ionization yield is very small, so a very weak REMPI is seen at $10.0 \mathrm{eV}$. The increase in the excitation comes from the first state of $\Pi_{u}$ symmetry, which is $10.1 \mathrm{eV}$ above the ground state. So the increase in excitation at $10 \mathrm{eV}$ is not surprising. It quite likely that strong REMPI may be seen when exactly $10.1 \mathrm{eV}$ photon is used.

Going from higher photon energies to lower ones, whenever the number of photon(s) needed for ionization increases (marked by dashed blue lines in the figure), a strong decrease in the ionization yield is seen, while excitation drops after some more decrease 


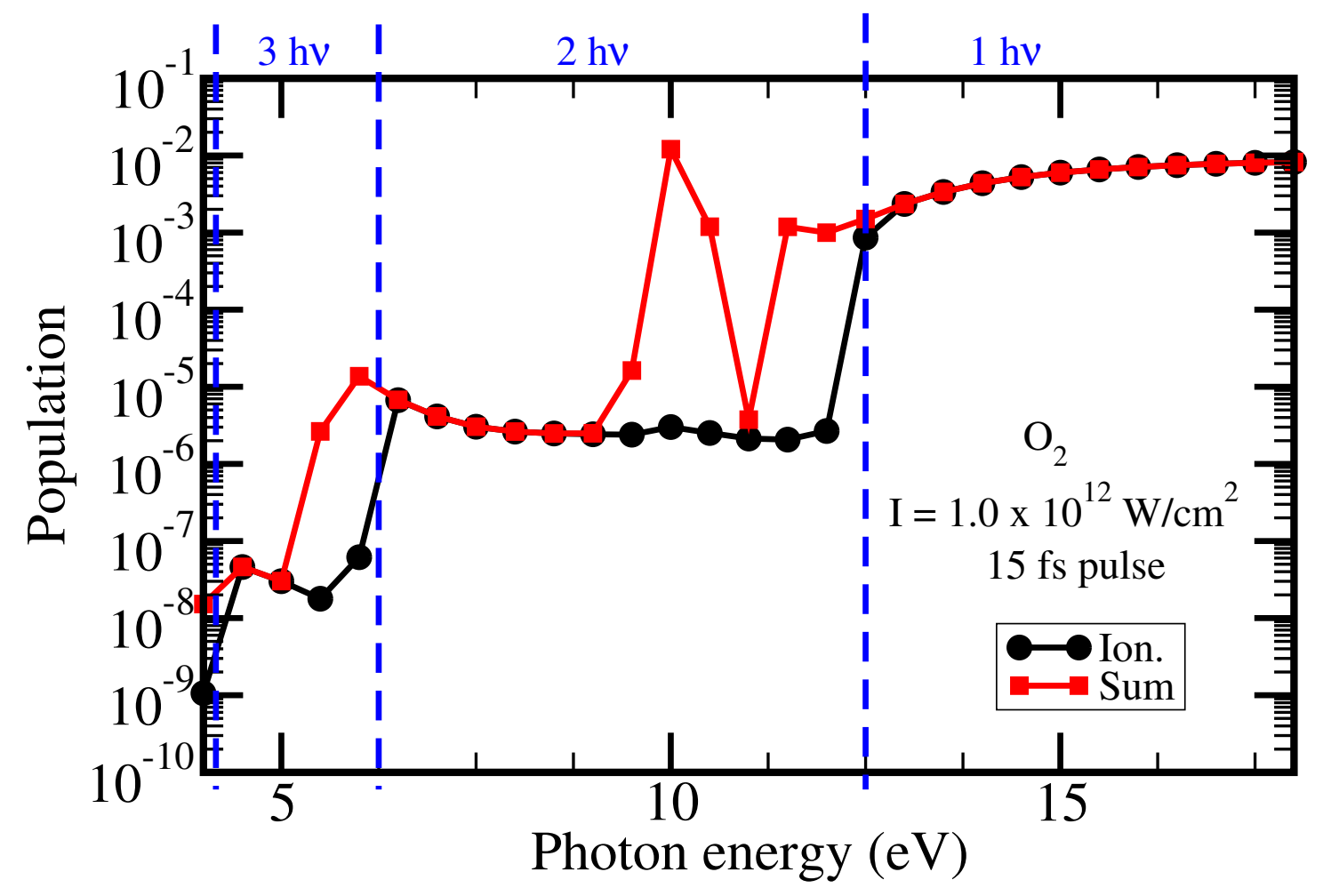

Figure 9.1: Ionization (black, circles) and sum of ionization and excitation (red, squares) as a function of photon energy for $\mathrm{O}_{2}$ molecule. Laser intensity is $1.0 \times$ $10^{12} \mathrm{~W} / \mathrm{cm}^{2}$ and $15 \mathrm{fs}$ pulse is used. The thresholds for n-photon ionization are marked by blue dashed lines.

in photon energy. The reason being that the (bound) Rydberg states are populated after the $\mathrm{n}$-photon ionization threshold. So some more decrease in photon energy is needed to depopulate or reduce the population of these closely lying Rydberg states.

Figure 9.2 is very similar to Figure 9.1, the only difference being that of the system under investigation. Figure 9.2 shows the photon energy scan for $\mathrm{N}_{2}$ molecule with the same laser parameters as used in Figure 9.1. The ionization potential for $\mathrm{N}_{2}$ as calculated by DFT-SAE is $15.92 \mathrm{eV}$. As in the case of $\mathrm{O}_{2}$, the symmetries involved here are $\Pi_{g}$ and $\Pi_{u}$, the difference being that the ground state here has $\Pi_{u}$ symmetry. Thus, overall symmetry of the ground state is even and no suppression should be observed as predicted by Muth-Böhm and coworkers [108, 189].

Unlike the case of $\mathrm{O}_{2}$, clear signatures of REMPI are seen. REMPI is seen at $4.5 \mathrm{eV}$, $6.0 \mathrm{eV}$ and $13.5 \mathrm{eV}$ (an increase in both ionization and excitation yield is seen). The REMPI at $4.5 \mathrm{eV}$ and $13.5 \mathrm{eV}$ is caused by the first state of $\Pi_{g}$ symmetry. This state is $13.46 \mathrm{eV}$ above the ground state energy. So this state can be reached by three photons of $4.5 \mathrm{eV}$ or by a single photon of $13.5 \mathrm{eV}$. The REMPI at $6.0 \mathrm{eV}$ is caused by the second state of $\Pi_{u}$ symmetry which is located $12.3 \mathrm{eV}$ above the ground state. So with two 


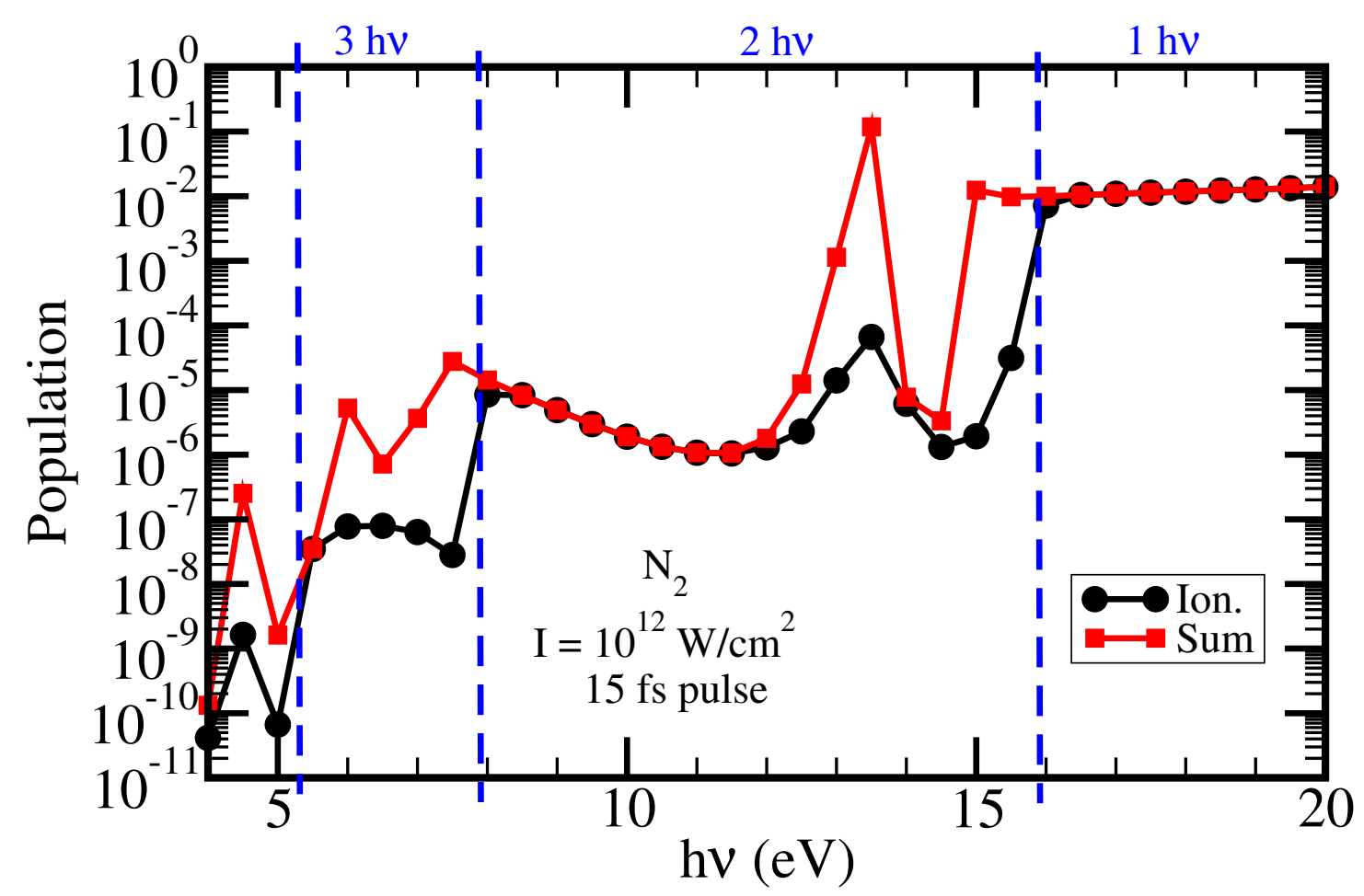

Figure 9.2: Same as Figure 9.1 but $\mathrm{N}_{2}$ molecule.

photons of $6.0 \mathrm{eV}$ one goes to $12.0 \mathrm{eV}$ and the state being close is also populated. Since the state is slightly off-resonance the REMPI at $6.0 \mathrm{eV}$ is not very strong (ionization yield is only slightly increased).

Here again, strong drop in ionization yield is seen when the number of photon(s) needed for ionization increases (see blue dashed lines in the figure).

Figure 9.3 is very similar to Figure 9.1, the only difference being that the system under investigation is $\mathrm{C}_{2} \mathrm{H}_{2}$. Figure 9.3 shows the photon energy scan for $\mathrm{C}_{2} \mathrm{H}_{2}$ molecule with the same laser parameters as used in Figure 9.1. The ionization potential for $\mathrm{C}_{2} \mathrm{H}_{2}$ as calculated by DFT-SAE is $12.27 \mathrm{eV}$. As in the case of $\mathrm{O}_{2}$, the symmetries involved here are $\Pi_{g}$ and $\Pi_{u}$. The acetylene molecule has four $\pi$ electrons in the degenerate highest occupied molecular orbital (HOMO). The symmetry of the ground state is $\Pi_{u}$.

As in the case $\mathrm{O}_{2}$ molecule, the REMPI in case of $\mathrm{C}_{2} \mathrm{H}_{2}$ is also weak. The weak REMPI (only substantial increase in excitation yield, without any significant increase in ionization yield) is seen at $5.0 \mathrm{eV}$ and $11.0 \mathrm{eV}$. The weak REMPI at $5.0 \mathrm{eV}$ comes from the second state of the $\Pi_{u}$ symmetry which is $9.84 \mathrm{eV}$ above the ground state. So a two-photon transition of $5.0 \mathrm{eV}$ photons from the ground state is close to resonance. The weak REMPI at $11.0 \mathrm{eV}$ comes from the second state of the $\Pi_{g}$ symmetry which is $11.11 \mathrm{eV}$ above the ground state. So a one-photon transition of $11.0 \mathrm{eV}$ photon from the ground state is close to resonance. A strong REMPI signal is seen at $6.5 \mathrm{eV}$ and it comes from first state of $\Pi_{g}$ symmetry and is located $6.69 \mathrm{eV}$ above the ground state. 


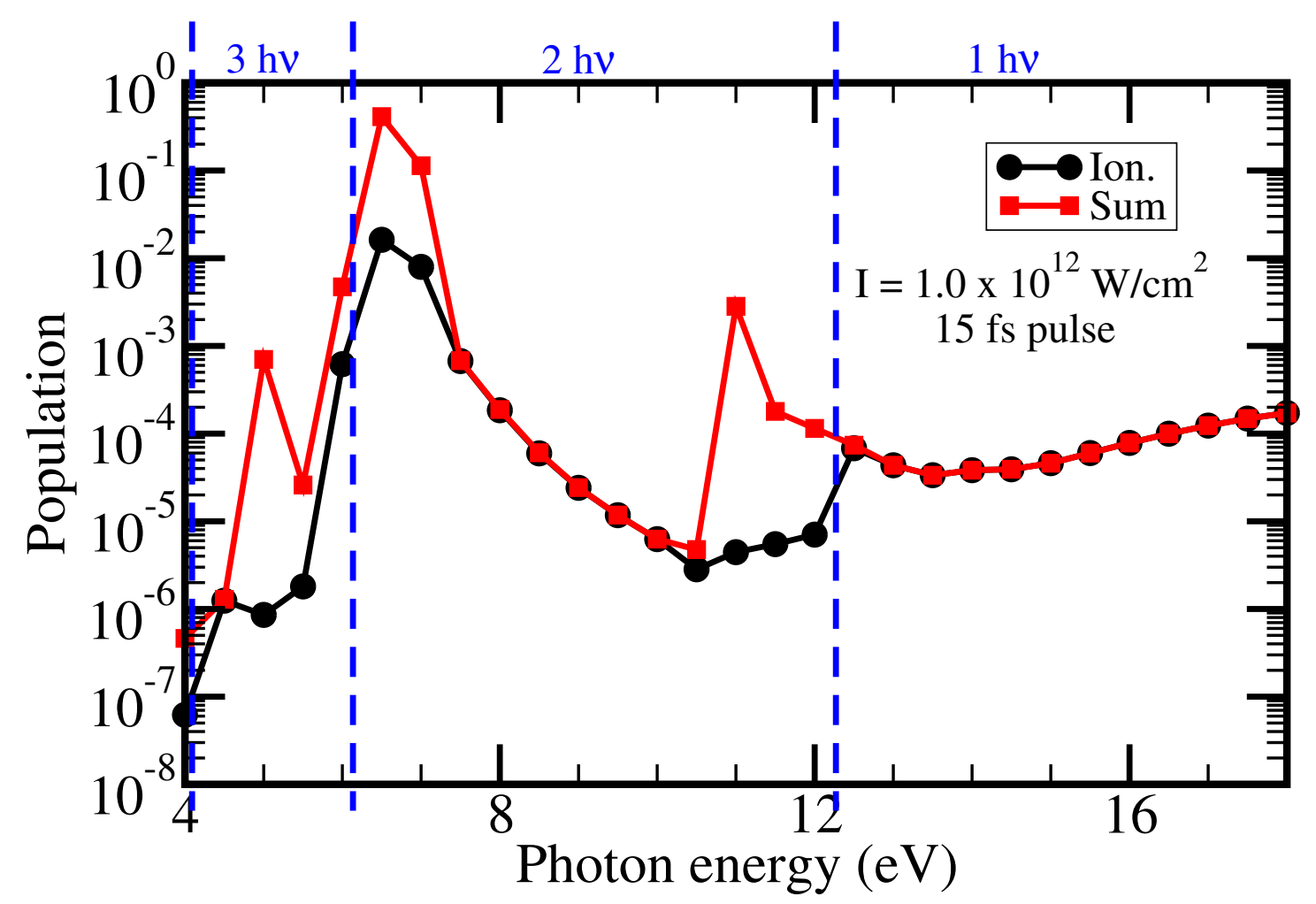

Figure 9.3: Same as Figure 9.1 but $\mathrm{C}_{2} \mathrm{H}_{2}$ molecule.

\section{$9.2800 \mathrm{~nm}$}

Figure 9.4 shows the ionization yield for $\mathrm{N}_{2}$ as a function of peak laser intensity. A $800 \mathrm{~nm}$ laser with 12 cycles ( $32 \mathrm{fs}$ ) is used. The yields are obtained by three methods, all based on SAE approximation. The DFT-SAE-TDSE method is already described in Chapter 3 and MOADK in Section 4.2. The results for SFA in velocity gauge were obtained in a private communication from Yulian Vanne. It should be noted that all input parameters going into MOADK and SFA calculations were obtained from the same basis set as used in TDSE calculation. Looking at curves, one sees that MOADK does not agree with DFT-SAE-TDSE calculation, while SFA in velocity gauge is in good agreement with the DFT-SAE-TDSE. There is just a slight disagreement at higher intensities between SFA and DFT-SAE-TDSE. SFA also reproduces channel closing, as seen by small dips in the curve. Thus, overall there is no model which agrees with TDSE calculation in the complete intensity range. Accurate calculations using multi-configuration HartreeFock [192-194] for $\mathrm{N}_{2}$ exist, but the results for absolute ionization yields have not been published so far.

Figure 9.5 shows ionization yield for $\mathrm{O}_{2}$ as a function of peak laser intensity. A $800 \mathrm{~nm}$ laser with 12 cycles ( $32 \mathrm{fs}$ ) is used. The yield obtained from DFT-SAE-TDSE method is compared to the yields obtained from the MOADK model. The yields for the MOADK 


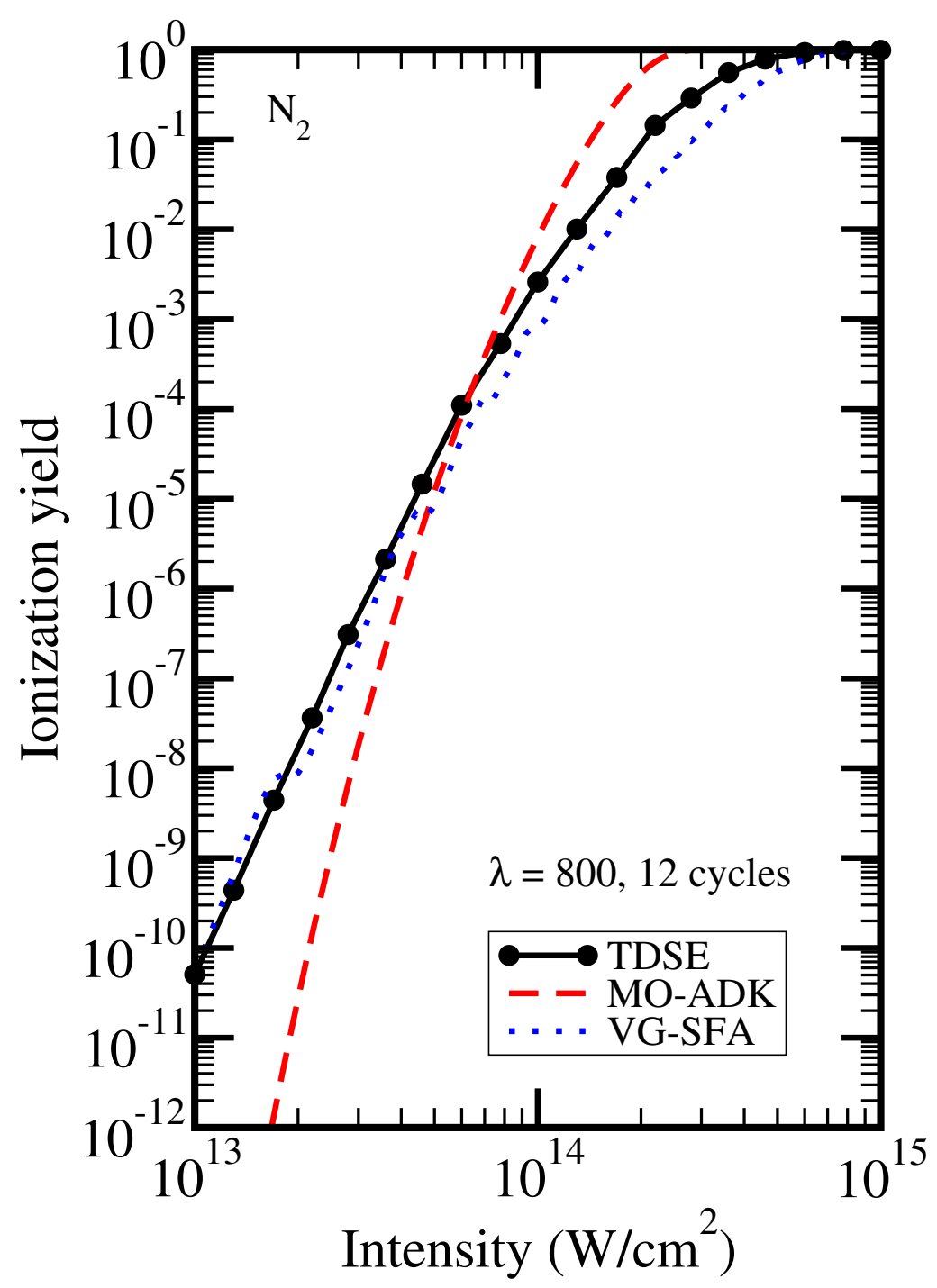

Figure 9.4: Ionization yield as a function of peak laser intensity for $\mathrm{N}_{2}$ is shown. Ionization yields are obtained by DFT-SAE-TDSE (black, circles), MOADK (red, dashed line) and velocity gauge SFA (blue, dots). $800 \mathrm{~nm}$ laser with 12 cycles (32 fs) is used.

model are calculated using different input parameters obtained from other references. The MOADK yield obtained from DFT-SAE LB94 functional is shown by the solid blue line. Since the same basis set is used for TDSE calculation, it should provide the best match. The MOADK yields obtained from parameters given by Tong et.al. [103] is shown by the red dotted line, while the yields obtained from parameters obtained by Madsen and co-workers [109] is shown by green dashed line. As seen from the figure, MOADK fails to agree with TDSE calculation. Even the slope provided by MOADK model is 


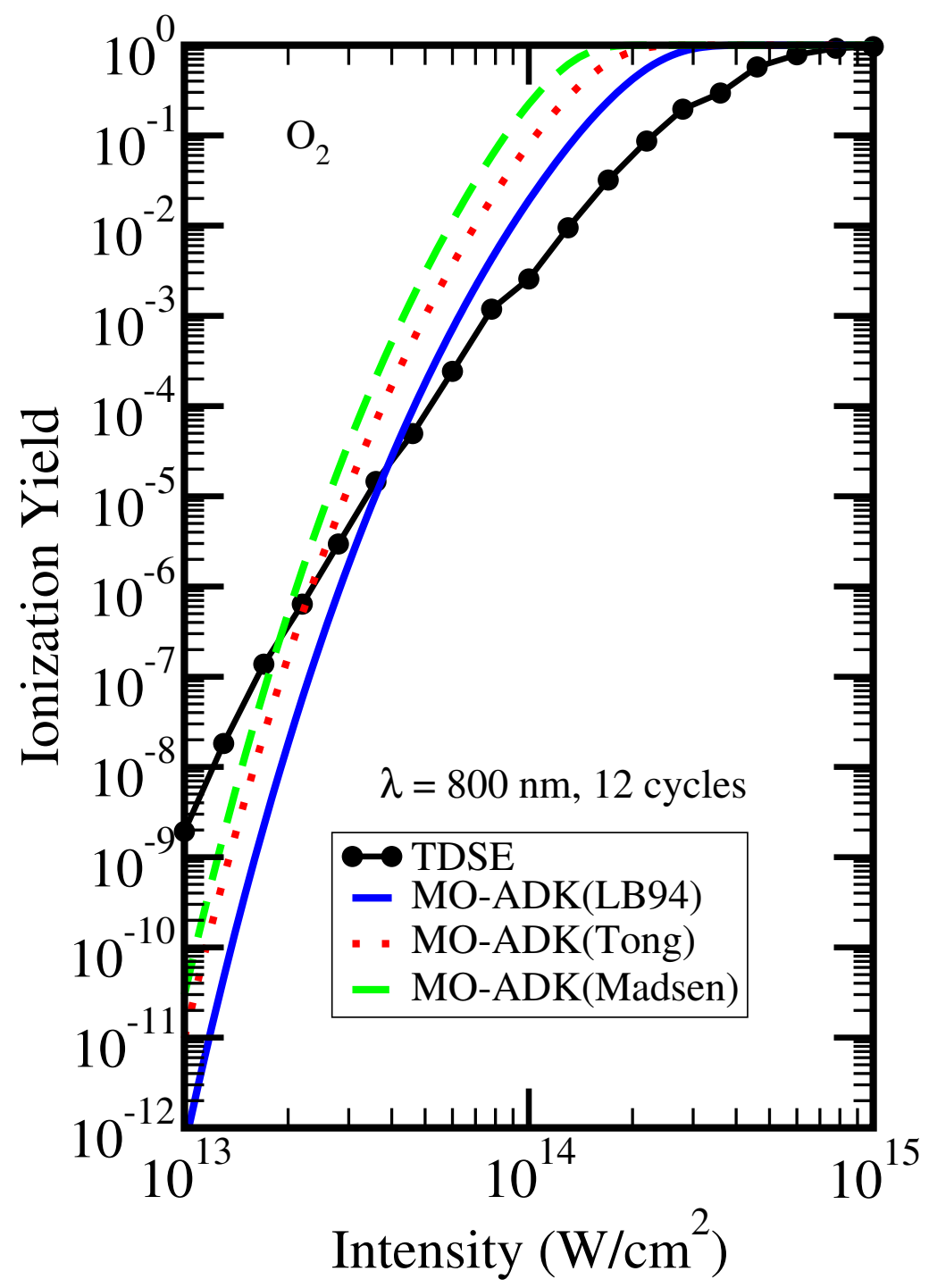

Figure 9.5: Ionization as function of peak intensity for $800 \mathrm{~nm}$ laser is shown. The MOADK yields are calculated from DFT-SAE using LB94 functional (blue, solid line), MOADK parameters for Tong are obtained from Reference [103] and Madsen from Reference [109].

incorrect. The parameters used in MOADK model are obtained by asymptotic fitting of the wavefunction at large distances. This fitting can be done in different ways, so there will always be discrepancy in the results, as seen in three MOADK results shown for $\mathrm{O}_{2}$.

Figure 9.6 shows the ionization and excitation yields for $\mathrm{C}_{2} \mathrm{H}_{2}$ molecule as a function of peak laser intensity. The results are for $800 \mathrm{~nm}$ laser with 12 cycles (32 fs). Ionization increases smoothly as intensity increase. The excitation shows structure due to the interplay between several bound states. The experimental results for the ionization of 


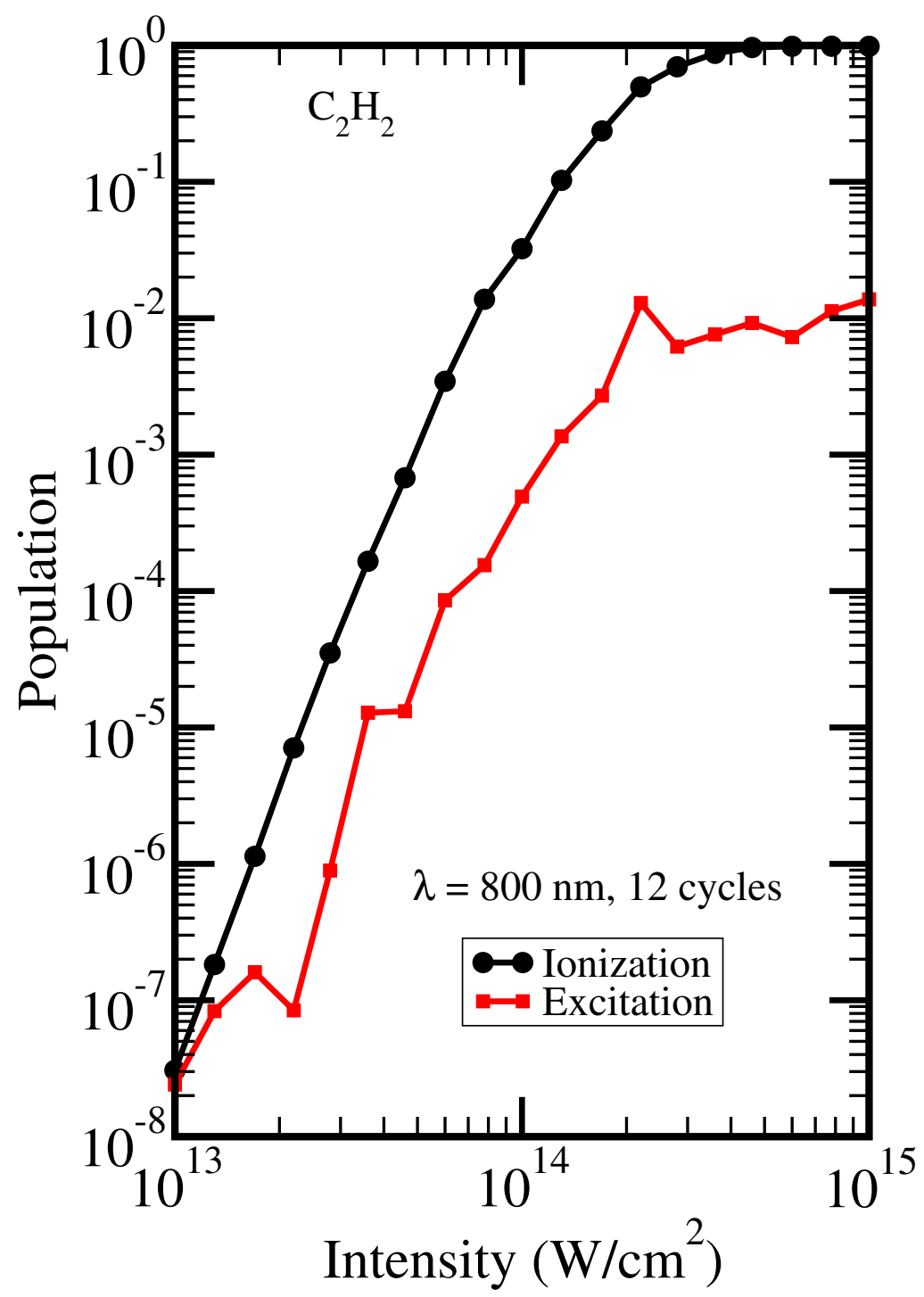

Figure 9.6: Ionization (black, circles) and excitation (red, squares) for $\mathrm{C}_{2} \mathrm{H}_{2}$ as a function of peak laser intensity. A $800 \mathrm{~nm}$ laser with 12 cycles (32 fs) is used.

acetylene already exist $[195,196]$. These results are for non-aligned $\mathrm{C}_{2} \mathrm{H}_{2}$ molecule and also averaged over the internuclear geometry (not at a fixed value of internuclear distance). In Reference [196] it was shown that the saturation intensity for $\mathrm{C}_{2} \mathrm{H}_{2}^{+}$ion is around $10^{14} \mathrm{~W} / \mathrm{cm}^{2}$. The saturation intensity obtained from the present calculation is $2.0 \times 10^{14} \mathrm{~W} / \mathrm{cm}^{2}$, which is quite close to the experimental value. A recent calculation based on DFT for $\mathrm{C}_{2} \mathrm{H}_{2}$ showed that ionization yield when the $\mathrm{C}_{2} \mathrm{H}_{2}$ molecule is aligned along the field is smaller when compared to the ionization yield when molecule is oriented perpendicular to the field [197]. With this result and angular averaging for the ionization yield, the saturation intensity for the un-aligned molecule if calculated in DFT-SAE- 
TDSE will be lower than $2.0 \times 10^{14} \mathrm{~W} / \mathrm{cm}^{2}$ and will be closer the experimental value of $10^{14} \mathrm{~W} / \mathrm{cm}^{2}$.

\section{$9.3400 \mathrm{~nm}$}

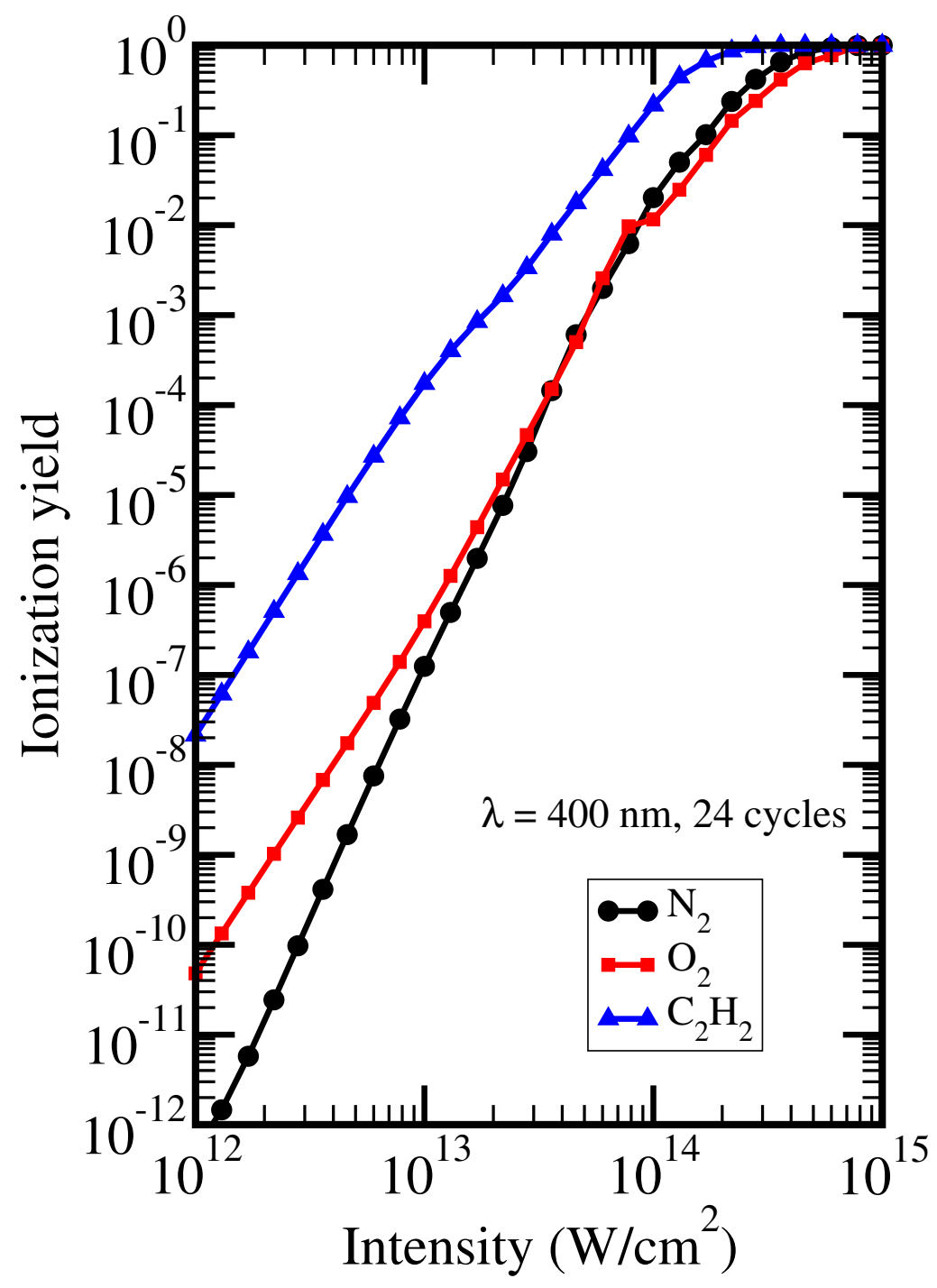

Figure 9.7: Ionization yield as a function of peak laser intensity for $\mathrm{N}_{2}$ (black, circles), $\mathrm{O}_{2}$ (red, squares) and $\mathrm{C}_{2} \mathrm{H}_{2}$ (blue, triangles) at $400 \mathrm{~nm}$. The pulse duration is 24 cycles ( $32 \mathrm{fs})$.

Figure 9.7 shows ionization yield for $\mathrm{N}_{2}, \mathrm{O}_{2}$ and $\mathrm{C}_{2} \mathrm{H}_{2}$ at $400 \mathrm{~nm}(31 . \mathrm{eV})$. A 24 cycle pulse (32 fs) with a cosine squared envelope for the vector potential (Equation 2.22) is used. The ionization yield for $\mathrm{C}_{2} \mathrm{H}_{2}$ molecule is highest at all intensities. At lower 
intensities the ionization yield for $\mathrm{O}_{2}$ is higher than that for $\mathrm{N}_{2}$, but as the intensity increases, the ionization yield for $\mathrm{O}_{2}$ drops down to below that for $\mathrm{N}_{2}$, showing signs of suppressed ionization. Here the suppression is even greater. It is not the suppression between molecule-atom pair with the same ionization potential. The suppression seen here is between two molecules having ionization potentials which approximately differ by the photon energy in this case. The difference between the ionization potentials is $3.43 \mathrm{eV}$ while the incident photon energy is $3.1 \mathrm{eV}$. Such high level of suppressed ionization in $\mathrm{O}_{2}$ at intensities above $10^{14} \mathrm{~W} / \mathrm{cm}^{2}$ at $400 \mathrm{~nm}$ is really remarkable. What is really interesting is that the suppression is intensity dependent. At the moment, to the knowledge of the author, experimental results comparing the two molecules at $400 \mathrm{~nm}$ do not exist. It will interesting to see the experimental results of ionization of $\mathrm{N}_{2}$ and $\mathrm{O}_{2}$ at this wavelength. 



\section{Summary}

Two methods for the solution of the time-dependent Schrödinger equation for molecules exposed to strong laser fields were successfully implemented and tested. Both methods use a superposition of field-free eigenstates for the expansion of the time-dependent electronic wavefunction. The first method (CI-TDSE) is applicable to two-electron systems $\left(\mathrm{H}_{2}\right.$ molecule or Helium atom) or effective two electron systems (alkali dimers). The CI-TDSE method uses configuration interaction for the description of two-electron or effective two-electron system. The second method (DFT-SAE-TDSE) is based on the single-active-electron (SAE) approximation and can be applied to larger molecules. The field-free electronic eigenstates are calculated using density-functional theory (DFT).

The CI-TDSE method has produced benchmark calculations for $\mathrm{H}_{2}$ molecule which have been further verified by other groups [118]. The method has successfully predicted ionization, excitation and photo-electron spectra including above-threshold ionization for the $\mathrm{H}_{2}$ molecule. The implementation in this work is limited to the fixed-nuclei approximation, but an arbitrary variation of the internuclear distance is possible. The work for including nuclear motion is also underway. Even an arbitrary orientation of the molecular axis relative to the laser polarization has been considered by the present approach [117], an important advantage to the other fully three-dimensional correlated approach based on a grid $[112,113]$.

The proper implementation of the code is demonstrated by comparing results obtained for extremely small internuclear distances with those of a very accurate calculation describing atomic helium exposed to ultrashort laser pulses. A second test comprises a comparison to the prediction of lowest-order perturbation theory (LOPT) that should be applicable for sufficiently low intensities. Increasing the peak intensity of the laser shows then first deviations from the behaviour predicted by LOPT, mainly due to the power broadening of resonant transitions and the ponderomotive shift leading to channel closing. While these conclusions are valid for the total ionization yield, the analysis of the photoelectron spectrum reveals even for the lower intensities the importance of nonperturbative effects. This indicates the importance of experiments yielding differential observables, but also the need to compare the predictions of different theoretical models on more detailed quantities than the total ionization yield.

The $R$ dependence of the ionization yield predicted by the quasi-static approximation including a vertical ionization potential was confirmed for $800 \mathrm{~nm}$ laser pulses with peak intensities between $3.5 \times 10^{13} \mathrm{~W} / \mathrm{cm}^{2}$ and $1.0 \times 10^{14} \mathrm{~W} / \mathrm{cm}^{2}$. However, only for the highest peak intensity a very good absolute agreement is found. For the lower intensities the ionization yield contains structure that is absent in the QSA model. There is also a large overall quantitative disagreement. The latter is, however, less important, if one is only interested in qualitative effects like the relative population of different vibrational 


\section{Summary}

states of $\mathrm{H}_{2}^{+}$formed from $\mathrm{H}_{2}$ in strong laser fields.

The validity of widely used single-active-electron (SAE) approximation and the models based on it is also discussed. The second method (DFT-SAE-TDSE) for the treatment of molecules exposed to intense laser fields is based on SAE. It describes all but one electron within density-functional theory (DFT). The resulting time-dependent one-electron Schrödinger equation describing the selected electron in the combined field of the frozen core and the laser pulse is solved numerically using an expansion in field-free eigenstates.

The developed approach was applied to molecular hydrogen and the calculated total ionization and excitation yields were compared to a full two-electron (CI-TDSE) calculation. For high photon frequencies and relatively low intensities it was demonstrated that the SAE model predicts reasonable results for the dependence of ionization and excitation on the photon frequency. However, due to the pronounced structure of the spectrum and the sensitivity of it on the ionization and excitation energies, the SAE results at a given photon energy may deviate drastically from the correct yields. In the weakly perturbative regime this failure can be compensated to a large extent by rescaling the photon frequency.

For the popular Ti:Sapphire laser wavelength of about $800 \mathrm{~nm}$ the intensity dependence of the ion yield obtained with different DFT functionals was investigated. From the tested ones, only LB94 and HF gave satisfactory results. In fact, the results obtained in these two cases agreed very well with each other and with the CI results. The agreement to CI is improved, if a factor 2 is adopted for laser intensities for which the ion yields are smaller than about $10 \%$. This factor should reflect the two equivalent electrons. The predicted excitation yield is also in reasonable agreement with the CI result, but here the agreement becomes even less good for high peak intensities for which the SAE models strongly overestimate excitation. Clearly, by construction SAE models do not account for double excitation or even double ionization.

At smaller wavelengths the spectra are more sensitive to structural details. At the considered photon energies that correspond to the 2nd and 3rd harmonic of the $800 \mathrm{~nm}$ radiation the erroneously predicted ionization energy of $\mathrm{H}_{2}$ using especially the LB94 model leads to channel-closing effects in the ion yield that are shifted in intensity compared to the CI result. The structural effects are much more pronounced in the excitation spectra and lead to differences between SAE and CI that can easily exceed some orders of magnitude.

In those cases where the HF and LB94 results differed, the HF results are usually in better overall agreement with CI. This should be a consequence of the well-known problem of DFT to describe systems with a very small number of electrons like atomic or molecular hydrogen. The failure of LB94 to describe $\mathrm{H}_{2}$ is already apparent from the poor ionization potential obtained with this approach. In fact, the very good overall agreement between HF and LB94 is somewhat surprising in view of these differences. With increasing size of the molecular system one should, however, expect that the present DFT-based approach should be superior to HF, since DFT is known to provide a very efficient way for a reliable description for a large number of many-electron molecules. An advantage of DFT in comparison to HF is the possibility to include to some extent correlation into the calculation. In the context of molecules in intense laser fields a 
prominent example for this advantage is given by molecular nitrogen. While HF predicts a wrong ordering of the orbitals and thus of the highest-occupied one, DFT predicts the correct ordering, as was also verified with the present code. More sophisticated functionals (such as LDA with self-interaction correction or exact-exchange within the optimized effective potential method) could be implemented in the future. The use of more sophisticated potentials does not increase the computational load of the timepropagation part of the calculation that is computationally most demanding, and is therefore merely a coding task.

The intensity-dependence of the ionization yield of $\mathrm{H}_{2}$ predicted by MO-ADK is found to be in very poor agreement with SAE-TDSE, even at the longest wavelength $(800 \mathrm{~nm})$ considered in this work. There are not only quantitative deviations, but also the slope of the curves differ considerably. Since it has been shown earlier that for field strengths lower than the classical threshold for over-the-barrier ionization ADK predicts absolute $d$ c-field ionization rates of $\mathrm{H}_{2}$ in very good agreement with a full complex-scaling based $a$ b initio CI calculation [86, 198], the observed failure of MO-ADK is due to a breakdown of the quasi-static approximation. This failure may not appear surprising in view of the fact that based on the analysis of Keldysh parameter and classical over-thebarrier ionization threshold limit the applicability of a tunneling model like ADK should be limited to a very small range of intensities. However, the present work provides a quantitative description of the failure of MO-ADK to approximate the SAE-TDSE result. This is very important, since MO-ADK was frequently used outside its parameter range of validity, for example in the context of suppressed ionization. The present work shows that such an analysis is very problematic.

After establishing the DFT-SAE-TDSE for $\mathrm{H}_{2}$ molecule, the DFT-SAE-TDSE approach was applied to larger molecules. The results for $\mathrm{N}_{2}, \mathrm{O}_{2}$ and $\mathrm{C}_{2} \mathrm{H}_{2}$ are shown and compared with other SAE models. The DFT-SAE-TDSE method confirms the prediction of ionization suppression in $\mathrm{O}_{2}$ and non-suppression in $\mathrm{N}_{2}$. As in the case of $\mathrm{H}_{2}$, the MO-ADK model fails to predict the correct ionization behaviour at $800 \mathrm{~nm}$, while the results of SFA give correct slope of the ionization yield and the predicted ionization yields are close to the ones predicted by DFT-SAE-TDSE. It should also be reminded that in contrast to SAE-TDSE none of the simplified SAE models MO-ADK or MO-SFA can predict excitation to electronic bound states. Especially for smaller wavelengths excitation is found to be very important. At the moment the DFT-SAE-TDSE method has been extended to include any arbitrary orientation of the laser molecule with respect to the laser polarization. The results for the orientation dependence of the ionization yield in $\mathrm{N}_{2}, \mathrm{O}_{2}$ and water molecules exist.

In depth study of the first excited state, $\mathrm{B}^{1} \Sigma_{u}^{+}$of $\mathrm{H}_{2}$ was also done. This study was mainly done using CI-TDSE approach. The SAE model that gets closest to the full calculation is the DFT-SAE-TDSE. This is surprising as the energy differences between the states obtained by CI and DFT-SAE are quite large. The p-SAE (CI-SAE) and KSAE do not agree with full CI-TDSE calculation. The failure of SAE approximation is not surprising for the $\mathrm{B}^{1} \Sigma_{u}^{+}$state, as all SAE models fail to include the ionic character which plays an important role in the ionization dynamics. For the case of ionization at standard wavelengths of $800 \mathrm{~nm}, 400 \mathrm{~nm}$ and $266 \mathrm{~nm}$ the intermediate states assume 


\section{Summary}

importance and signatures of REMPI are seen. The interesting prediction from the quasistatic approximation at $R=1.4 a_{0}$ is also verified. A channel crossing between $\mathrm{B}^{1} \Sigma_{u}^{+}$ and $\mathrm{E}, \mathrm{F}^{1} \Sigma_{g}^{+}$states was seen. The diabatic picture derived from adiabatic calculation provided a much better explanation of the time-dependent results.

The present work is not complete and a lot is needed to be done in the future. Inclusion of the nuclear motion, calculation of the high order harmonic spectra and angle resolved photoelectron spectra are just a few of them. 


\section{Abbreviations}

\begin{tabular}{ll} 
Abbreviation & Explanation \\
\hline ADK & Ammosov-Delone-Krainov \\
CI-TDSE & $\begin{array}{l}\text { Configuration interaction } \\
\text { Configuration interaction based method for solution of } \\
\text { time-dependent Schrödinger equation }\end{array}$ \\
COLTRIMS & Cold Target Recoil Ion Momentum Spectroscopy \\
CREI & Charge resonance enhanced ionization \\
DFT & Density functional theory \\
DFT-SAE-TDSE & Density-functional theory based method for solution of \\
& time-dependent Schrödinger equation within \\
FEL & single-active-electron approximation \\
FTI & Free electron laser \\
HOHG & Frustrated tunnel ionization \\
MO-ADK & High order harmonic generation \\
OESE & Molecular orbital Ammosov-Delone-Krainov \\
REMPI & One-electron Schrödinger equation \\
SAE & Resonance enhanced multi-photon ionization \\
SFA-LG & Single-active-electron \\
SFA-VG & Strong field approximation in length gauge \\
SFA & Strong field approximation in velocity gauge \\
TDSE & Strong field approximation \\
TESE & Time-dependent Schrödinger equation \\
TISE & Two-electron Schrödinger equation \\
\hline & Time-independent Schrödinger equation \\
\hline
\end{tabular}





\section{Acknowledgments}

First and foremost I offer my sincerest gratitude to my supervisor, PD Dr. Alejandro Saenz, who has supported me throughout my thesis with his patience and knowledge whilst allowing me the room to work in my own way. I attribute the level of my $\mathrm{PhD}$ degree to his encouragement and effort and without him this thesis, too, would not have been completed or written. One simply could not wish for a better or friendlier supervisor. Working under his supervision is also a great learning experience. Apart from physics, I learned organizational and communication skills from him.

I would like to thank my family for standing by my side and supporting me in all possible ways during my $\mathrm{PhD}$. Even though they were far away, but I could feel their support across the oceans.

I would also like to thank the AMO group members for the fruitful discussions and stimulating group meetings. A special thanks to Yulian V. Vanne for his help and expertise in the field. I am grateful to the members of NANO and QOM groups for keeping me socially involved in the activities.

The DFT-SAE-TDSE project is collaboration between three groups under the COST framework. I would like to thank Prof. Piero Decleva and Dr. Alberto Castro for their guidance in this project.

The help and encouragement from my M.Sc. class-mates kept me motivated. The wide range of physical problems discussed over emails enhanced my general knowledge of physics. I am grateful to Vishwesha Guttal and Arvind Ayyer for the proof-reading of my thesis.

Life in Berlin was made a lot easier because of my Indian friends. I never felt away from India whenever I was with them. Their help in practical issues is invaluable. I should specially thank my apartment-mates, Anand Sharma and Mahesh Bansal, for bearing me over a long period of time.

The list of people to be thanked for the thesis is endlessly long. I can not mention all their names but I express my sincere heartfelt gratitude to all of them.

Finally I would like to thank Deutsche Forschungsgemeinschaft (DFG), Sonderforschungsbereich 450 (SFB 450) and Humboldt Universität for providing the financial support for my $\mathrm{PhD}$. 



\section{Bibliography}

[1] Nasr A. M. Hafz, Tae Moon Jeong, Il Woo Choi, Seong Ku Lee, Ki Hong Pae, Victor V. Kulagin, Jae Hee Sung, Tae Jun Yu, Kyung-Han Hong, Tomonao Hosokai, John R. Cary, Do-Kyeong Ko, and Jongmin Lee. Stable generation of GeV-class electron beams from self-guided laser-plasma channels. Nature Photonics, 2:571, 2008 .

[2] J. Itatani, J. Levesque, D. Zeidler, Hiromichi Niikura, H. Pépin, J. C. Kieffer, P. B. Corkum, and D. M. Villeneuve. Tomographic imaging of molecular orbitals. Nature, 432:867, 2004.

[3] Thomas Brabec and Ferenc Krausz. Intense few-cycle laser fields: Frontiers of nonlinear optics. Rev. Mod. Phys., 72:545, 2000.

[4] G. G. Paulus, F. Lindner, H. Walther, A. Baltuška, E. Goulielmakis, M. Lezius, and F. Krausz. Measurement of the phase of few-cycle laser pulses. Phys. Rev. Lett., 91:253004, 2003.

[5] A. Baltuška, Th. Udem, M. Uiberacker, M. Hentschel, E. Goulielmakis, Ch. Gohle, R. Holzwarth, V. S. Yakovlev, A. Scrinzi, T. W. Hänsch, and F. Krausz. Attosecond control of electronic processes by intense light fields. Nature, 421:611, 2003.

[6] Vladimir Roudnev, B.D. Esry, and I. Ben-Itzhak. Controlling $\mathrm{HD}^{+}$and $\mathrm{H}_{2}^{+}$dissociation with the carrier-envelope phase difference of an intense ulatrshort laser pulse. Phys. Rev. Lett., 93:163601, 2004.

[7] X. M. Tong and C. D. Lin. Carrier-envelope phase dependence of nonsequential double ionization of $\mathrm{H}_{2}$ by few-cycle laser pulses. J. Phys. B: At. Mol. Phys., 40: 641, 2007.

[8] M. Y. Shverdin, D. R. Walker, D. D. Yavuz, G. Y. Yin, and S. E. Harris. Generation of a single-cycle optical pulse. Phys. Rev. Lett., 94:033904, 2005.

[9] M. Hentschel, R. Kienberger, Ch. Spielmann, G. A. Reider, N. Milosevic, T. Brabec, P. Corkum, U. Heinzmann, M. Drescher, and F. Krausz. Attosecond metrology. Nature, 414:509, 2001.

[10] Nenad Milosevic, Armin Scrinzi, and Thomas Brabec. Numerical characterization of high harmonic attosecond pulses. Phys. Rev. Lett., 88:093905, 2002. 
[11] G. Sansone, E. Benedetti, F. Calegari, C. Vozzi, L. Avaldi, R. Flammini, L. Poletto, P. Villoresi, C. Altucci, R. Velotta, S. Stagira, S. De Silvestri, and M. Nisoli. Isolated single-cycle attosecond pulses. Science, 314:443, 2006.

[12] J. Ullrich and H. Schmidt-Böcking. Time-of-flight spectrometer for the determination of microradian projectile scattering angles in atomic collisions. Phys. Lett. A, 125:193, 1987.

[13] C. L. Cocke and R. E. Olson. Recoil ions. Physics Reports, 205:153, 1991.

[14] J. Ullrich, R. Moshammer, R. Dörner, O. Jagutzki, V. Mergel, H. Schmidt-Böcking, and L. Spielberger. Recoil-ion momentum spectroscopy. J. Phys. B: At. Mol. Phys., 30:2917, 1997.

[15] M. Dörr and R. M. Potvliege. Adiabatic stabilization in photodetachment by ultrashort pulses. J. Phys. B: At. Mol. Phys., 33:L233, 2000.

[16] C. J. Joachain, M. Dörr, and N. Kylstra. High-intensity laser-atom physics. Advances in Atomic, Molecular, and Optical Physics, 42:225, 2000.

[17] J. Ullrich, R. Moshammer, A. Dorn, R. Dörner, L. Ph. H. Schmidt, and H. SchmidtBöcking. Recoil-ion and electron momentum spectroscopy: reaction-microscopes. Rep. Prog. Phys., 66:1463, 2003.

[18] J. H. Posthumus. The dynamics of small molecules in intense laser fields. Rep. Prog. Phys., 67:623, 2004.

[19] Th. Ergler, A. Rudenko, B. Feuerstein, K. Zrost, C. D. Schröter, R. Moshammer, and J. Ullrich. Spatiotemporal imaging of ultrafast molecular motion: Collapse and revival of the $\mathrm{D}_{2}^{+}$nuclear wave packet. Phys. Rev. Lett., 97:193001, 2006.

[20] A. Rudenko, Th. Ergler, B. Feuerstein, K. Zrost, C.D. Schröter, R. Moshammer, and J. Ullrich. Real-time observation of vibrational revival in the fastest molecular system. Chem. Phys., 329:193, 2006.

[21] Hiromichi Niikura, F. Légaré, R. Hasbani, A. D. Bandrauk, Misha Yu. Ivanov, D. M. Villeneuve, and P. B. Corkum. Sub-laser-cycle electron pulses for probing molecular dynamics. Nature, 417:917, 2002.

[22] Hiromichi Niikura, F. Légaré, R. Hasbani Misha Yu. Ivanov, D. M. Villeneuve, and P. B. Corkum. Probing molecular dynamics with attosecond resolution using correlated wave packet pairs. Nature, 421:826, 2003.

[23] C. D. Lin, X. M. Tong, and Toru Morishita. Direct experimental visualization of atomic and electron dynamics with attoseond pulses. J. Phys. B: At. Mol. Phys., 39:S419, 2006.

[24] L. V. Keldysh. Ionization in the field of a strong electromagnetic wave. Sov. Phys. JETP, 20:1307, 1965. 
[25] M. Ferray, A. L'Huillier, X. F. Li, L. A. Lompre, G. Mainfray, and C. Manus. Multiple-harmonic conversion of $1064 \mathrm{~nm}$ radiation in rare gases. J.Phys. B: At. Mol. Phys., 21:L31, 1988.

[26] X. F. Li, A. L'Huillier, M. Ferray, L. A. Lompré, and G. Mainfray. Multipleharmonic generation in rare gases at high laser intensity. Phys. Rev. A, 39:5751, 1989.

[27] Jeffrey L. Krause, Kenneth J. Schafer, and Kenneth C. Kulander. High-order harmonic generation from atoms and ions in the high intensity regime. Phys. Rev. Lett., 68:3535, 1992.

[28] A. L'Huillier, K. J. Schafer, and K. C. Kulander. Theoretical aspects of intense field harmonic generation. J. Phys. B: At. Mol. Phys., 24:3315, 1991.

[29] P. Agostini, F. Fabre, G. Mainfray, G. Petite, and N. K. Rahman. Free-free transitions following six-photon ionization of xenon atoms. Phys. Rev. Lett., 42: $1127,1979$.

[30] P. Kruit, J. Kimman, and M. J. Van der Wiel. Absorption of additional photons in the multiphoton ionisation continuum of Xenon at 1064, 532 and $440 \mathrm{~nm}$. J. Phys. B: At. Mol. Phys., 14:L597, 1981.

[31] P. Kruit, J. Kimman, H. G. Muller, and M. J. van der Wiel. Electron spectra from multiphoton ionization of xenon at 1064, 532, and $355 \mathrm{~nm}$. Phys. Rev. A, 28:248, 1983.

[32] R. R. Freeman, P. H. Bucksbaum, H. Milchberg, S. Darack, D. Schumacher, and M. E. Geusic. Above-threshold ionization with subpicosecond laser pulses. Phys. Rev. Lett., 59:1092, 1987.

[33] M. Pont and M. Gavrila. Stabilization of atomic hydrogen in superintense, highfrequency laser fields of circular polarization. Phys. Rev. Lett., 65:2362, 1990.

[34] Kenneth C. Kulander, Kenneth J. Schafer, and Jeffrey L. Krause. Dynamic stabilization of hydrogen in an intense, high-frequency, pulsed laser field. Phys. Rev. Lett., 66:2601, 1991.

[35] M. P. de Boer, J. H. Hoogenraad, R. B. Vrijen, L. D. Noordam, and H. G. Muller. Indications of high-intensity adiabatic stabilization in neon. Phys. Rev. Lett., 71: 3263, 1993.

[36] J. H. Eberly and K. C. Kulander. Atomic Stabilization by Super-Intense Lasers. Science, 262:1229, 1993.

[37] Bretislav Freidrich and Dudley R. Herschbach. Spatial orientation of molecules in strong electric fields and evidence for pendular states. Nature, 353:412, 1991. 
[38] Bretislav Friedrich and Dudley Herschbach. Alignment and trapping of molecules in intense laser fields. Phys. Rev. Lett., 74:4623, 1995.

[39] Juan Ortigoso, Mirta Rodríguez, Manish Gupta, and Bretislav Friedrich. Time evolution of pendular states created by the interaction of molecular polarizability with a pulsed nonresonant laser field. J. Chem. Phys., 110:3870, 1999.

[40] Tamar Seideman. Revival structure of aligned rotational wave packets. Phys. Rev. Lett., 83:4971, 1999.

[41] Tamar Seideman. On the dynamics of rotationally broad, spatially aligned wave packets. J. Chem. Phys., 115:5965, 2001.

[42] Long Cai, Jotin Marango, and Bretislav Friedrich. Time-dependent alignment and orientation of molecules in combined electrostatic and pulsed nonresonant laser fields. Phys. Rev. Lett., 86:775, 2001.

[43] Mette Machholm. Postpulse alignment of molecules robust to thermal averaging. J. Chem. Phys., 115:10724, 2001.

[44] Henrik Stapelfeldt and Tamar Seideman. Colloquium: Aligning molecules with strong laser pulses. Rev. Mod. Phys., 75:543, 2003.

[45] F. Rosca-Pruna and M. J. J. Vrakking. Experimental observation of revival structures in picosecond laser-induced alignment of $\mathrm{I}_{2}$. Phys. Rev. Lett., 87:153902, 2001.

[46] F. Rosca-Pruna, E. Springate, H. L. Offerhaus, M. Krishnamurthy, N. Farid, C. Nicole, and M. J. J. Vrakking. Spatial alignment of diatomic molecules in intense laser fields: I. Experimental results. J.Phys. B: At. Mol. Phys., 34:4919, 2001.

[47] I. V. Litvinyuk, Kevin F. Lee, P. W. Dooley, D. M. Rayner, D. M. Villeneuve, and P. B. Corkum. Alignment-dependent strong field ionization of molecules. Phys. Rev. Lett., 90:233003, 2003.

[48] P. W. Dooley, I. V. Litvinyuk, Kevin F. Lee, D. M. Rayner, M. Spanner, D. M. Villeneuve, and P. B. Corkum. Direct imaging of rotational wave-packet dynamics of diatomic molecules. Phys. Rev. A, 68:023406, 2003.

[49] M. Comstock, V. V. Lozovoy, and M. Dantus. Rotational wavepacket revivals for phase modulation of ultrafast pulses. Chem. Phys. Lett., 372:739, 2003.

[50] V. Renard, M. Renard, S. Guérin, Y. T. Pashayan, B. Lavorel, O. Faucher, and H. R. Jauslin. Postpulse molecular alignment measured by a weak field polarization technique. Phys. Rev. Lett., 90:153601, 2003.

[51] V. Renard, M. Renard, A. Rouzée, S. Guérin, H. R. Jauslin, B. Lavorel, and O. Faucher. Nonintrusive monitoring and quantitative analysis of strong laserfield-induced impulsive alignment. Phys. Rev. A, 70:033420, 2004. 
[52] P. H. Bucksbaum, A. Zavriyev, H. G. Muller, and D. W. Schumacher. Softening of the $\mathrm{H}_{2}^{+}$molecular bond in intense laser fields. Phys. Rev. Lett., 64:1883, 1990.

[53] A. Giusti-Suzor, F. H. Mies, L. F. DiMauro, E. Charron, and B. Yang. Dynamics of $\mathrm{H}_{2}^{+}$in intense laser fields. J. Phys. B: At. Mol. Phys., 28:309, 1995.

[54] A. D. Bandrauk and M. L. Sink. Photodissociation in intense laser fields: Predissociation analogy. J. Chem. Phys., 74:1110, 1981.

[55] John R. Hiskes. Dissociation of molecular ions by electric and magnetic fields. Phys. Rev., 122:1207, 1961.

[56] Alejandro Saenz. Behavior of molecular hydrogen exposed to strong dc, ac, or low-frequency laser fields: I. Bond softening and enhanced ionization. Phys. Rev. A, 66:063407, 2002.

[57] A. Giusti-Suzor and F. H. Mies. Vibrational trapping and suppression of dissociation in intense laser fields. Phys. Rev. Lett., 68:3869, 1992.

[58] Guanhua Yao and Shih-I Chu. Laser-induced molecular stabilization and trapping and chemical bond hardening in intense laser fields. Chem. Phys. Lett., 197:413, 1992.

[59] Guanhua Yao and Shih-I Chu. Molecular-bond hardening and dynamics of molecular stabilization and trapping in intense laser pulses. Phys. Rev. A, 48:485, 1993.

[60] Eric E. Aubanel, Jean-Marc Gauthier, and André D. Bandrauk. Molecular stabilization and angular distribution in photodissociation of $\mathrm{H}_{2}^{+}$in intense laser fields. Phys. Rev. A, 48:2145, 1993.

[61] Sarah W. Allendorf and Abraham Szöke. High-intensity multiphoton ionization of $\mathrm{H}_{2}$. Phys. Rev. A, 44:518, 1991.

[62] A. Zavriyev, P. H. Bucksbaum, J. Squier, and F. Saline. Light-induced vibrational structure in $\mathrm{H}_{2}^{+}$and $\mathrm{D}_{2}^{+}$in intense laser fields. Phys. Rev. Lett., 70:1077, 1993.

[63] K. Codling and L. J. Frasinski. Dissociative ionization of small molecules in intense laser fields. J. Phys. B: At. Mol. Phys., 26:783, 1993.

[64] M. Schmidt, D. Normand, and C. Cornaggia. Laser-induced trapping of chlorine molecules with pico- and femtosecond pulses. Phys. Rev. A, 50:5037, 1994.

[65] J. H. Posthumus, L. J. Frasinski, A. J. Giles, and K. Codling. Dissociative ionization of molecules in intense laser fields: a method of predicting ion kinetic energies and appearance intensities. J. Phys. B: At. Mol. Phys., 28:L349, 1995.

[66] Tamar Seideman, M. Yu. Ivanov, and P. B. Corkum. Role of electron localization in intense-field molecular ionization. Phys. Rev. Lett., 75:2819, 1995. 
[67] T. Zuo and A. D. Bandrauk. Charge-resonance-enhanced ionization of diatomic molecular ions by intense lasers. Phys. Rev. A, 52:R2511, 1995.

[68] T. D. G. Walsh, F. A. Ilkov, and S. L. Chin. The dynamical behaviour of $\mathrm{H}_{2}$ and $\mathrm{D}_{2}$ in a strong, femtosecond, titanium:sapphire laser field. J. Phys. B: At. Mol. Phys., 30:2167, 1997.

[69] G. N. Gibson, M. Li, C. Guo, and J. Neira. Strong-field dissociation and ionization of $\mathrm{H}_{2}^{+}$using ultrashort laser pulses. Phys. Rev. Lett., 79:2022, 1997.

[70] L. J. Frasinski, J. H. Posthumus, J. Plumridge, K. Codling, P. F. Taday, and A. J. Langley. Manipulation of bond hardening in $\mathrm{H}_{2}^{+}$by chirping of intense femtosecond laser pulses. Phys. Rev. Lett., 83:3625, 1999.

[71] J. H. Posthumus, J. Plumridge, L. J. Frasinski, K. Codling, E. J. Divall, A. J. Langley, and P. F. Taday. Slow protons as a signature of zero-photon dissociation of $\mathrm{H}_{2}^{+}$in intense laser fields. J. Phys. B: At. Mol. Phys., 33:L563, 2000.

[72] D. Normand, C. Cornaggia, and J. Morellec. Six-photon ionisation of $\mathrm{H}_{2}$ via the $\mathrm{E}, \mathrm{F}^{1} \Sigma_{g}^{+}, \nu_{\mathrm{E}}=0$ level. Absorption of photons in the ionisation continuum. J. Phys. B: At. Mol. Phys., 19:2881, 1986.

[73] J. W. J. Verschuur, L. D. Noordam, and H. B. van Linden van den Heuvell. Anomalies in above-threshold ionization observed in $\mathrm{H}_{2}$ and its excited fragments. Phys. Rev. A, 40:4383, 1989.

[74] A. Giusti-Suzor, X. He, O. Atabek, and F. H. Mies. Above-threshold dissociation of $\mathrm{H}_{2}^{+}$in intense laser fields. Phys. Rev. Lett., 64:515, 1990.

[75] Mark Thachuk, M. Yu. Ivanov, and David M. Wardlaw. A semiclassical approach to intense-field above-threshold dissociation in the long wavelength limit. II. Conservation principles and coherence in surface hopping. J. Chem. Phys., 109:5747, 1998.

[76] I. D. Williams, P. McKenna, B. Srigengan, I. M. G. Johnston, W. A. Bryan, J. H. Sanderson, A. El-Zein, T. R. J. Goodworth, W. R. Newell, P. F. Taday, and A. J. Langley. Fast-beam study of $\mathrm{H}_{2}^{+}$ions in an intense femtosecond laser field. J. Phys. B: At. Mol. Phys., 33:2743, 2000.

[77] I. Ben-Itzhak, P. Q. Wang, J. F. Xia, A. M. Sayler, M. A. Smith, K. D. Carnes, and B. D. Esry. Dissociation and ionization of $\mathrm{H}_{2}^{+}$by ultrashort intense laser pulses probed by coincidence 3d momentum imaging. Phys. Rev. Lett., 95:073002, 2005.

[78] J. McKenna, A. M. Sayler, F. Anis, B. Gaire, Nora G. Johnson, E. Parke, J. J. Hua, H. Mashiko, C. M. Nakamura, E. Moon, Z. Chang, K. D. Carnes, B. D. Esry, and I. Ben-Itzhak. Enhancing high-order above-threshold dissociation of $\mathrm{H}_{2}^{+}$ beams with few-cycle laser pulses. Phys. Rev. Lett., 100:133001, 2008. 
[79] K. Codling, L. J. Frasinski, and P. A. Hatherly. On the field ionisation of diatomic molecules by intense laser fields. J. Phys. B: At. Mol. Phys., 22:L321, 1989.

[80] Zufar Mulyukov, Marcel Pont, and Robin Shakeshaft. Ionization, dissociation, and level shifts of $\mathrm{H}_{2}^{+}$in a strong dc or low-frequency ac field. Phys. Rev. A, 54:4299, 1996.

[81] M. Plummer and J. F. McCann. Orientation dependence of field ionization of the hydrogen molecular ion. J. Phys. B: At. Mol. Phys., 30:L401, 1997.

[82] Szczepan Chelkowski, André Conjusteau, Tao Zuo, and André D. Bandrauk. Dissociative ionization of $\mathrm{H}_{2}^{+}$in an intense laser field: Charge-resonance-enhanced ionization, Coulomb explosion, and harmonic generation at $600 \mathrm{~nm}$. Phys. Rev. A, 54:3235, 1996.

[83] A. D. Bandrauk and Jonathan Ruel. Charge-resonance enhanced ionization of molecular ions in intense laser pulses: Geometric and orientation effects. Phys. Rev. A, 59:2153, 1999 .

[84] K. Codling, L. J. Frasinski, and P. A. Hatherly. Multiphoton ionisation of $\mathrm{H}_{2}$ and $\mathrm{D}_{2}$ using an intense sub-picosecond laser. J. Phys. B: At. Mol. Phys., 21:L433, 1988.

[85] E. Constant, H. Stapelfeldt, and P. B. Corkum. Observation of enhanced ionization of molecular ions in intense laser fields. Phys. Rev. Lett., 76:4140, 1996.

[86] A. Saenz. Enhanced ionization of molecular hydrogen in very strong fields. Phys. Rev. A, 61:051402(R), 2000.

[87] I. Ben-Itzhak, P. Q. Wang, A. M. Sayler, K. D. Carnes, M. Leonard, B. D. Esry, A. S. Alnaser, B. Ulrich, X. M. Tong, I. V. Litvinyuk, C. M. Maharjan, P. Ranitovic, T. Osipov, S. Ghimire, Z. Chang, and C. L. Cocke. Elusive enhanced ionization structure for $\mathrm{H}_{2}^{+}$in intense ultrashort laser pulses. Phys. Rev. A, 78: $063419,2008$.

[88] Hengtai Yu and André D. Bandrauk. Three-dimensional Cartesian finite element method for the time dependent Schrödinger equation of molecules in laser fields. J. Chem. Phys., 102:1257, 1995.

[89] R. Kopold, W. Becker, and M. Kleber. Model calculations of high-harmonic generation in molecular ions. Phys. Rev. A, 58:4022, 1998.

[90] B. Zimmermann, M. Lein, and J.M. Rost. Analysis of recombination in high-order harmonic generation in molecules. Phys. Rev. A, 71:033401, 2005.

[91] D. G. Lappas and J. P. Marangos. Orientation dependence of high-order harmonic generation in hydrogen molecular ions. J. Phys. B: At. Mol. Phys., 33:4679, 2000. 
[92] M. Lein, N. Hay, R. Velotta, J. P. Marangos, and P. L. Knight. Role of the intramolecular phase in high-harmonic generation. Phys. Rev. Lett., 88:183903, 2002.

[93] M. Lein, P. P. Corso, J. P. Marangos, and P. L. Knight. Orientation dependence of high-order harmonic generation in molecules. Phys. Rev. A, 67:023819, 2003.

[94] W. Boutu, S. Haessler, H. Merdji, P. Breger, G. Waters, M. Stankiewicz, L. J. Frasinski, R. Taieb, J. Caillat, A. Maquet, P. Monchicourt, B. Carre, and P. Salieres. Coherent control of attosecond emission from aligned molecules. Nature Physics, 4:545, 2008.

[95] M. Lein, N. Hay, R. Velotta, J. P. Marangos, and P. L. Knight. Interfence effects in high-order harmonic generation with molecules. Phys. Rev. A, 66:023805, 2002.

[96] G. Lagmago Kamta and A. D. Bandrauk. High-order harmonic generation from two-center molecules: Time-profile analysis of nuclear contributions. Phys. Rev. A, 70:011404(R), 2004.

[97] Tsuneto Kanai, Shinichirou Minemoto, and Hirofumi Sakai. Quantum interference during high-order harmonic generation from aligned molecules. Nature, 435:470, 2005 .

[98] Anh-Thu Le, X.-M. Tong, and C. D. Lin. Evidence of two-center interference in high-order harmonic generation from $\mathrm{CO}_{2}$. Phys. Rev. A, 73:041402, 2006.

[99] C. Vozzi, F. Calegari, E. Benedetti, J. P. Caumes, S. Stagira, M. Nisoli, R. Torres, E. Heesel, N. Kajumba, J. P. Marangos, C. Altucci, and R. Velotta. Controlling two-center interference in molecular high harmonic generation. Phys. Rev. Lett., 95:153902, 2005.

[100] Z. Ansari, M. Böttcher, B. Manschwetus, H. Rottke, W. Sandner, A. Verhoef, M. Lezius, G. G. Paulus, A. Saenz, and D B Milošević. Interference in strong-field ionization of a two-centre atomic system. New J. Phys., 10:093027, 2008.

[101] Szczepan Chelkowski, Tao Zuo, Osman Atabek, and André D. Bandrauk. Dissociation, ionization, and Coulomb explosion of $\mathrm{H}_{2}^{+}$in an intense laser field by numerical integration of the time-dependent Schrödinger equation. Phys. Rev. A, 52:2977, 1995.

[102] M. V. Ammosov, N. B. Delone, and V. P. Krainov. Tunnel ionization of complex atoms and of atomic ions in an alternating electromagnetic field. Journal of Experimental and Theoretical Physics, 64:1191, 1986.

[103] X. M. Tong, Z. X. Zhao, and C. D. Lin. Theory of molecular tunneling ionization. Phys. Rev. A, 66:033402, 2002.

[104] X. M. Tong, Z. X. Zhao, and C. D. Lin. Probing molecular dynamics at attosecond resolution with femtosecond laser pulses. Phys. Rev. Lett., 91:233203, 2003. 
[105] X. Urbain, B. Fabre, E. M. Staicu-Casagrande, N. de Ruette, V. M. Andrianarijaona, J. Jureta, J. H. Posthumus, A. Saenz, E. Baldit, and C. Cornaggia. Intenselaser-field ionization of molecular hydrogen in the tunneling regime and its effect on the vibrational excitation of $\mathrm{H}_{2}^{+}$. Phys. Rev. Lett., 92:163004, 2004.

[106] Amalia Apalategui and Alejandro Saenz. Multiphoton ionization of the hydrogen molecule $\mathrm{H}_{2}$. J. Phys. B: At. Mol. Phys., 35:1909, 2002.

[107] A. Becker and F. H. M. Faisal. Intense-field many-body S-matrix theory. J. Phys. B: At. Mol. Phys., 38:R1, 2005.

[108] J. Muth-Böhm, A. Becker, and F. H. M. Faisal. Suppressed molecular ionization for a class of diatomics in intense femtosecond laser fields. Phys. Rev. Lett., 85: $2280,2000$.

[109] Thomas Kim Kjeldsen and Lars Bojer Madsen. Strong-field ionization of diatomic molecules and companion atoms: Strong-field approximation and tunneling theory including nuclear motion. Phys. Rev. A, 71:023411, 2005.

[110] S. Chelkowski, C. Foisy, and A. D. Bandrauk. Electron-nuclear dynamics of multiphoton $\mathrm{H}_{2}^{+}$dissociative ionization in intense laser fields. Phys. Rev. A, 57:1176, 1998 .

[111] Hiromichi Niikura, D. M. Villeneuve, and P. B. Corkum. Mapping attosecond electron wave packet motion. Phys. Rev. Lett., 94:083003, 2005.

[112] Kenji Harumiya, Isao Kawata, Hirohiko Kono, and Yuichi Fujimura. Exact twoelectron wave packet dynamics of $\mathrm{H}_{2}$ in an intense laser field: Formation of localized ionic states $\mathrm{H}^{+} \mathrm{H}^{-}$. J. Chem. Phys., 113:8953, 2000.

[113] Hirohiko Kono, Isao Kawata, and Yuichi Fujimura. Development of dual transformation method and its application to electronic and nuclear dynamics in intense laser fields. RIKEN Review, 29:60, 2000.

[114] Kenji Harumiya, Hirohiko Kono, Yuichi Fujimura, Isao Kawata, and A. D. Bandrauk. Intense laser-field ionization of $\mathrm{H}_{2}$ enhanced by two-electron dynamics. Phys. Rev. A, 66:043403, 2002.

[115] P. Lambropoulos, P. Maragakis, and Jian Zhang. Two-electron atoms in strong fields. Phys. Rep., 305:203, 1998.

[116] S. Barmaki, H. Bachau, and M. Ghalim. Dissociation and ionization dynamics of $\mathrm{H}_{2}^{+}$with short laser pulses: The $\mathrm{L}^{2}$ approach. Phys. Rev. A, 69:043403, 2004.

[117] Y. V. Vanne and A. Saenz. Ionization of $\mathrm{H}_{2}$ in intense ultrashort laser pulses: parallel versus perpendicular orientation. J. Mod. Opt., 55:2665, 2008.

[118] A. Palacios, H. Bachau, and F. Martín. Excitation and ionization of molecular hydrogen by ultrashort vuv laser pulses. Phys. Rev. A, 75:013408, 2007. 
[119] I. Barth, J. Manz, and G. K. Paramonov. Time-dependent extension of Koopmans' picture for ionisation by a laser pulse: application to $\mathrm{H}_{2}\left(\mathrm{~B}^{1} \Sigma_{u}^{+}\right)$. Mol. Phys., 106: 467, 2008.

[120] L. Wolniewicz. Relativistic energies of the ground state of the hydrogen molecule. J. Chem. Phys., 99:1851, 1993.

[121] Jacek Rychlewski, Wojciech Cencek, and Jacek Komasa. The equivalence of explicitly correlated Slater and Gaussian functions in variational quantum chemistry computations. the ground state of $\mathrm{H}_{2}$. Chem. Phys. Lett., 229:657, 1994.

[122] H. Bachau, E Cormier, P Decleva, J. E. Hansen, and F Martín. Applications of B-splines in atomic and molecular physics. Rep. Prog. Phys., 64:1815, 2001.

[123] Yulian V. Vanne and Alejandro Saenz. Numerical treatment of diatomic twoelectron molecules using a B-spline based CI method. J. Phys. B: At. Mol. Phys., $37: 4101,2004$.

[124] Nina Rohringer, Ariel Gordon, and Robin Santra. Configuration-interaction-based time-dependent orbital approach for ab initio treatment of electron dynamics in a strong optical field. Phys. Rev. A, 74:043420, 2006.

[125] Kenneth C. Kulander. Multiphoton ionization of hydrogen: A time-dependent theory. Phys. Rev. A, 35:445, 1987.

[126] X. Tang, H. Rudolph, and P. Lambropoulos. Nonperturbative time-dependent theory of helium in a strong laser field. Phys. Rev. A, 44:R6994, 1991.

[127] M. J. Nandor, M. A. Walker, L. D. Van Woerkom, and H. G. Muller. Detailed comparison of above-threshold-ionization spectra from accurate numerical integrations and high-resolution measurements. Phys. Rev. A, 60:R 1771, 1999.

[128] Rolf Wiehle, Bernd Witzel, Hanspeter Helm, and Eric Cormier. Dynamics of strong-field above-threshold ionization of argon: Comparison between experiment and theory. Phys. Rev. A, 67:063405, 2003.

[129] M. Lezius, V. Blanchet, D. M. Rayner, D. M. Villeneuve, Albert Stolow, and Misha Yu. Ivanov. Nonadiabatic multielectron dynamics in strong field molecular ionization. Phys. Rev. Lett., 86:51, 2001.

[130] Thomas Brabec, Michel Côté, Paul Boulanger, and Lora Ramunno. Theory of tunnel ionization in complex systems. Phys. Rev. Lett., 95:073001, 2005.

[131] V. R. Bhardwaj, P. B. Corkum, and D. M. Rayner. Internal laser-induced dipole force at work in $\mathrm{C}_{60}$ molecule. Phys. Rev. Lett., 91:203004, 2003.

[132] A. Jaroń-Becker, A. Becker, and F. H. M. Faisal. Saturated ionization of fullerenes in intense laser fields. Phys. Rev. Lett., 96:143006, 2006. 
[133] Hirohiko Kono, Yukio Sato, Nobuyuki Tanaka, Tsuyoshi Kato, Katsunori Nakai, Shiro Koseki, and Yuichi Fujimura. Quantum mechanical study of electronic and nuclear dynamics of molecules in intense laser fields. Chem. Phys., 304:203, 2004.

[134] Manohar Awasthi, Yulian V. Vanne, and Alejandro Saenz. Non-perturbative solution of the time-dependent Schrödinger equation describing $\mathrm{H}_{2}$ in intense short laser pulses. J. Phys. B: At. Mol. Phys., 38:3973, 2005.

[135] Manohar Awasthi and Alejandro Saenz. Internuclear-distance dependence of ionization of $\mathrm{H}_{2}$ in strong laser fields. J. Phys. B: At. Mol. Phys., 39:S 389, 2006.

[136] D. Toffoli, M. Stener, G. Fronzoni, and P. Decleva. Convergence of the multicenter B-spline DFT approach for the continuum. Chem. Phys., 276:25, 2002.

[137] E. J. Baerends, D. E. Ellis, and P. Ros. Self-consistent molecular Hartree-FockSlater calculations I. The computational procedure. Chem. Phys., 2:41, 1973.

[138] G. Fonseca-Guerra, J. G. Snijders, G. te Velde, and E. J. Baerends. Towards an order-N DFT method. Theor. Chem. Acc., 99:391, 1998.

[139] M. Brosolo and P. Decleva. Variational approach to continuum orbitals in a spline basis: an application to $\mathrm{H}_{2}^{+}$photoionization. Chem. Phys., 159:185, 1992.

[140] P. Hohenberg and W. Kohn. Inhomogeneous electron gas. Phys. Rev., 136:B 864, 1964.

[141] L. Wilk S. H. Vosko and M. Nusair. Accurate spin-dependent electron liquid correlation energies for local spin density calculations: a critical analysis. Can. J. Phys., $58: 1200,1980$.

[142] R. van Leeuwen and E. J. Baerends. Exchange-correlation potential with correct asymptotic behavior. Phys. Rev. A, 49:2421, 1994.

[143] M. E. McHenry, R. C. O'Handley, and K. H. Johnson. Physical significance of local-density-functional-theory eigenvalues. Phys. Rev. B, 35:3555, 1987.

[144] M. Stener, S. Furlan, and P. Decleva. Density functional calculations of photoionization with an exchange-correlation potential with the correct asymptotic behaviour. J. Phys. B: At. Mol. Phys., 33:1081, 2000.

[145] Thomas K. Kjeldsen, Christer Z. Bisgaard, Lars Boyer Madsen, and Henrik Stapelfeldt. Influence of molecular symmetry on strong-field ionization : Studies on ethylene, benzene, fluorebenzene and chlorofluorobenzene. Phys. Rev. A, 71: 013418, 2005.

[146] A. Talebpour, C.-Y. Chien, and S. L. Chin. The effects of dissociative recombination in multiphoton ionization of $\mathrm{O}_{2}$. J. Phys. B: At. Mol. Phys., 29:L677, 1996. 
Bibliography

[147] C. Guo, M. Li, J. P. Nibarger, and G. N. Gibson. Single and double ionization of diatomic molecules in strong laser fields. Phys. Rev. A, 58:R4271, 1998.

[148] S. M. Hankin, D. M. Villeneuve, P. B. Corkum, and D. M. Rayner. Nonlinear ionization of organic molecules in high intensity laser fields. Phys. Rev. Lett., 84: $5082,2000$.

[149] Jian Wu, Heping Zeng, and Chunlei Guo. Comparison study of atomic and molecular single ionization in the multiphoton ionization regime. Phys. Rev. Lett., 96: $243002,2006$.

[150] F. H. M. Faisal. Multiple absorption of laser photons by atoms. J. Phys. B: At. Mol. Phys., 6:L89, 1973.

[151] Howard R. Reiss. Effect of an intense electromagnetic field on a weakly bound system. Phys. Rev. A, 22:1786, 1980.

[152] E. P. Benis, J. F. Xia, X. M. Tong, M. Faheem, M. Zamkov, B. Shan, P. Richard, and Z. Chung. Ionization suppression of $\mathrm{Cl}_{2}$ molecules in intense laser fields. Phys. Rev. A, 70:025401, 2004.

[153] Thomas Kim Kjeldsen and Lars Bojer Madsen. Strong-field ionization of $\mathrm{N}_{2}$ : length and velocity gauge strong-field approximation and tunnelling theory. J. Phys. B: At. Mol. Phys., 37:2033, 2004.

[154] Thomas Kim Kjeldsen and Lars Bojer Madsen. Comment on "Strong-field ionization of laser-irradiated light homonuclear diatomic molecules: A generalized strong-field approximation-combination of atomic orbitals model". Phys. Rev. A, 73:047401, 2006.

[155] A. Jaroń-Becker, A. Becker, and F. H. M. Faisal. Ionization of $\mathrm{N}_{2}, \mathrm{O}_{2}$, and linear carbon clusters in a strong laser pulse. Phys. Rev. A, 69:023410, 2004.

[156] Vladimir I. Usachenko. Reply to "Comment on 'Strong-field ionization of laser-irradiated light homonuclear diatomic molecules: A generalized strong-field approximation-combination of atomic orbitals model' ". Phys. Rev. A, 73:047402, 2006 .

[157] D. B. Milošević. Strong-field approximation for ionization of a diatomic molecule by a strong laser field. Phys. Rev. A, 74:063404, 2006.

[158] A. M. Perelomov, V. S. Popov, and M. V. Terent'ev. Ionization of atoms in an alternating electric field. Sov. Phys. JETP, 23:924, 1966.

[159] J. Purvis, M. Dörr, M. Terao-Dunseath, C. J. Joachain, P. G. Burke, and C. J. Noble. Multiphoton ionization of $\mathrm{H}^{-}$and He in intense laser fields. Phys. Rev. Lett., 71:3943, 1993. 
[160] Armin Scrinzi and Bernard Piraux. Ionization and double excitation of helium by short intense laser pulses. Phys. Rev. A, 56:R13, 1997.

[161] Armin Scrinzi and Bernard Piraux. Two-electron atoms in short intense laser pulses. Phys. Rev. A, 58:1310, 1998.

[162] P. Lambropoulos, M. A. Kornberg, L. A. A. Nikolopoulos, and Alejandro Saenz. $\mathrm{Ab}$ initio approaches to multiphoton processes in two electron atoms. In L. F. DiMauro, R. R. Freeman, and K. C. Kulander, editors, Multiphoton Processes, page 231, New York, 2000. Melville.

[163] P. Maragakis, E. Cormier, and P. Lambropoulos. Time-dependent calculation of ionization in potassium at midinfrared wavelength. Phys. Rev. A, 60:4718, 1999.

[164] Alejandro Saenz. Electronically excited states of molecular hydrogen exposed to strong direct current, alternative current, or low-frequency laser fields. J. Phys. B: At. Mol. Phys., 35:4829, 2002.

[165] T. Nubbemeyer, K. Gorling, A. Saenz, U. Eichmann, and W. Sandner. Strong-field tunneling without ionization. Phys. Rev. Lett., 101:233001, 2008.

[166] B. Manschwetus, T. Nubbemeyer, K. Gorling, G. Steinmeyer, U. Eichmann, H. Rottke, and W. Sandner. Strong laser field fragmentation of $\mathrm{H}_{2}$ : Coulomb explosion without double ionization. Phys. Rev. Lett., 102:113002, 2009.

[167] Thomas Kim Kjeldsen and Lars Bojer Madsen. Vibrational excitation of diatomic molecular ions in strong field ionization of diatomic molecules. Phys. Rev. Lett., 95:073004, 2005.

[168] E. Goll, G. Wunner, and A. Saenz. Formation of ground-state vibrational wavepackets in intense ultrashort laser pulses. Phys. Rev. Lett., 97:103003, 2006.

[169] Th. Ergler, B. Feuerstein, A. Rudenko, K. Zrost, C. D. Schröter, R. Moshammer, and J. Ullrich. Quantum-phase resolved mapping of ground-state vibrational $\mathrm{D}_{2}$ wave packets via selective depletion in intense laser pulses. Phys. Rev. Lett., 97: 103004, 2006.

[170] M. Lezius, V. Blanchet, Misha Yu. Ivanov, and Albert Stolow. Polyatomic molecules in strong laser fields: Nonadiabatic multielectron dynamics. J. Chem. Phys., 117:1575, 2002.

[171] A. S. Alnaser, C. M. Maharjan, P. Wang, and I. V. Litvinyuk. Multi-photon resonant effects in strong-field ionization: origin of the dip in experimental longitudinal momentum distributions. J. Phys. B: At. Mol. Phys., 39:L323, 2006.

[172] Manohar Awasthi, Yulian V. Vanne, Alejandro Saenz, Alberto Castro, and Piero Decleva. Single-active-electron approximation for describing molecules in ultrashort laser pulses and its application to molecular hydrogen. Phys. Rev. A, 77: 063403, 2008. 
Bibliography

[173] A. Saenz. On the influence of vibrational motion on strong-field ionization rates in molecules. J. Phys. B: At. Mol. Phys., 33:4365, 2000.

[174] A. Requate, A. Becker, and F. H. M. Faisal. S-matrix theory of inelastic vibronic ionization of molecules in intense laser fields. Phys. Rev. A, 73:033406, 2006.

[175] Manfred Lein. Attosecond probing of vibrational dynamics with high-harmonic generation. Phys. Rev. Lett., 94:053004, 2005.

[176] S. Baker, J. S. Robinson, C. A. Haworth, H. Teng, R. A. Smith, C. C. Chirilă, M. Lein, J. W. G. Tisch, and J. P. Marangos. Probing Proton Dynamics in Molecules on an Attosecond Time Scale. Science, 312:424, 2006.

[177] M. Meckel, D. Comtois, D. Zeidler, A. Staudte, D. Pavičić, H. C. Bandulet, H. Pépin, J. C. Kieffer, R. Dörner, D. M. Villeneuve, and P. B. Corkum. Laserinduced electron tunneling and diffraction. Science, 320:1478, 2008.

[178] Xi Chu and Shih-I Chu. Role of the electronic structure and multielectron response in ionization mechanisms of diatomic molecules in intense short-pulse lasers: An all-electron ab initio study. Phys. Rev. A, 70:061402(R), 2004.

[179] G. Lagmago Kamta and A. D. Bandrauk. Imaging electron molecular orbitals via ionization by intense femtosecond pulses. Phys. Rev. A, 74:033415, 2006.

[180] Domagoj Pavičić, Kevin F. Lee, D. M. Rayner, P. B. Corkum, and D. M. Villeneuve. Direct measurement of the angular dependence of ionization for $\mathrm{N}_{2}, \mathrm{O}_{2}$, and $\mathrm{CO}_{2}$ in intense laser fields. Phys. Rev. Lett., 98:243001, 2007.

[181] A. Talebpour, S. Larochelle, and S. L. Chin. Multiphoton ionization of unsaturated hydrocarbons. J. Phys. B: At. Mol. Phys., 31:2769, 1998.

[182] Isao Kawata, Hirohiko Kono, Yuichi Fujimura, and André D. Bandrauk. Intenselaser-field-enhanced ionization of two-electron molecules: Role of ionic states as doorway states. Phys. Rev. A, 62:031401(R), 2000.

[183] A. Talebpour, S. Larochelle, and S. L. Chin. Suppressed tunnelling ionization of the $\mathrm{D}_{2}$ molecule in an intense Ti:sapphire laser pulse. J. Phys. B: At. Mol. Phys., 31:L49, 1998.

[184] Merrick J. DeWitt and Robert J. Levis. Calculating the Keldysh adiabaticity parameter for atomic and polyatomic molecules. J. Chem. Phys., 108:7739, 1998.

[185] A. Saenz. Molecular hydrogen exposed to a suddenly turned-on strong electric field or low-frequency laser. J. Phys. B: At. Mol. Phys., 33:3519, 2000.

[186] Chunlei Guo. Multielectron effects on single-electron strong field ionization. Phys. Rev. Lett., 85:2276, 2000. 
[187] F. Grasbon, G. G. Paulus, S. L. Chin, H. Walther, J. Muth-Böhm, A. Becker, and F. H. M. Faisal. Signatures of symmetry-induced quantum-interference effects observed in above-threshold-ionization spectra of molecules. Phys. Rev. A, 63: 041402(R), 2001.

[188] Merrick J. DeWitt, E. Wells, and R. R. Jones. Radiometric comparison of intense field ionization of atoms and diatomic molecules. Phys. Rev. Lett., 87:153001, 2001.

[189] J. Muth-Böhm, A. Becker, S. L. Chin, and F. H. M. Faisal. S-matrix theory of ionisation of polyatomic molecules in an intense laser pulse. Chem. Phys. Lett., 337:313, 2001.

[190] S. M. Hankin, D. M. Villeneuve, P. B. Corkum, and D. M. Rayner. Intense-field laser ionization rates in atoms and molecules. Phys. Rev. A, 64:013405, 2001.

[191] V. R. Bhardwaj, D. M. Rayner, D. M. Villeneuve, and P. B. Corkum. Quanten interference in double ionization and fragmentation of $\mathrm{C}_{6} \mathrm{H}_{6}$ in intense laser fields. Phys. Rev. Lett., 87:253003, 2001.

[192] Jürgen Zanghellini, Markus Kitzler, Thomas Brabec, and Armin Scrinzi. Testing the multi-configuration time-dependent Hartree-Fock method driven by a synthesized laser field. J. Phys. B: At. Mol. Phys., 37:763, 2004.

[193] J. Caillat, J. Zanghellini, O. Kitzler, O. Koch, W. kreuzer, and A. Scrinzi. Correlated multielectron systems in strong laser fields : A multiconfiguration timedependent hartree-fock approach. Phys. Rev. A, 71:012712, 2005.

[194] G. Jordan, J. Caillat, C. Ede, and A. Scrinzi. Strong field ionization of linear molecules: a correlated three-dimensional calculation. J. Phys. B: At. Mol. Phys., 39:S341, 2006.

[195] C. Cornaggia and Ph Hering. Laser-induced non-sequential double ionization of small molecules. J. Phys. B: At. Mol. Phys., 31:L503, 1998.

[196] C. Cornaggia and Ph. Hering. Nonsequential double ionization of small molecules induced by a femtosecond laser field. Phys. Rev. A, 62:023403, 2000.

[197] T. Otobe and K. Yabana. Density-functional calculation for the tunnel ionization rate of hydrocarbon molecules. Phys. Rev. A, 75:062507, 2007.

[198] Alejandro Saenz. Behavior of molecular hydrogen exposed to strong dc, ac, or lowfrequency laser fields: II. Comparison of ab initio and Ammosov-Delone-Krainov (ADK) rates. Phys. Rev. A, 66:063408, 2002. 



\section{List of Figures}

1.1 Ionization regimes on the basis of Keldysh parameter . . . . . . . . . . . 2

1.2 Ionization process in quasi-static regime . . . . . . . . . . . . 3

2.1 One-electron system . . . . . . . . . . . . . . . . . . . . . . 13

2.2 Solution of the one-electron Schrödinger equation for $\mathrm{H}_{2}^{+} \ldots \ldots \ldots$

2.3 The pulse shapes implemented in the code . . . . . . . . . . . . . . . . 20

4.1 Three photon ionization process . . . . . . . . . . . . . . 33

5.1 Ionization and excitation as a function of incident photon energy for He,

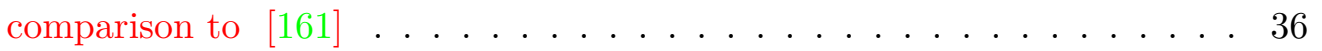

5.2 Ionization and excitation as a function of incident photon energy for He, comparison to $[162] \ldots \ldots \ldots \ldots \ldots \ldots$

5.3 Ionization as function of the peak laser intensity for He, comparison to LOPT . . . . . . . . . . . . . . . . . . . . 38

5.4 Ionization yield as a function of incident photon energy for $\mathrm{H}_{2}$, comparison

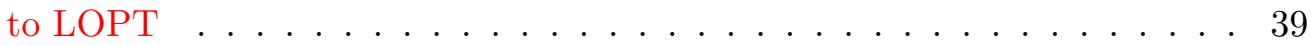

6.1 Ionization and sum of ionization and excitation for $\mathrm{H}_{2}$ as a function of incident photon energy . . . . . . . . . . . . . . . . 42

6.2 Ionization and excitation as a function of peak laser intensity for $\mathrm{H}_{2}(R=$ $\left.1.4 a_{0}\right)$ at $800 \mathrm{~nm} \ldots \ldots \ldots \ldots \ldots \ldots$

6.3 Ionization yield as a function of the internuclear distance. $800 \mathrm{~nm}$ laser with a peak intensity of $3.5 \times 10^{13} \mathrm{~W} / \mathrm{cm}^{2} \ldots \ldots \ldots \ldots$

6.4 Ionization yield as a function of the internuclear distance. $800 \mathrm{~nm}$ laser with a peak intensity of $5.4 \times 10^{13} \mathrm{~W} / \mathrm{cm}^{2} \ldots \ldots \ldots$. . . . . . 46

6.5 Ionization yield as a function of the internuclear distance. $800 \mathrm{~nm}$ laser with a peak intensity of $7.8 \times 10^{13} \mathrm{~W} / \mathrm{cm}^{2} \ldots \ldots \ldots$. . . . . . 46

6.6 Ionization yield as a function of the internuclear distance. $800 \mathrm{~nm}$ laser with a peak intensity of $1.06 \times 10^{14} \mathrm{~W} / \mathrm{cm}^{2} \ldots \ldots \ldots 47$

6.7 Ionization and excitation as a function of peak laser intensity for $\mathrm{H}_{2}(R=$ $\left.1.4 a_{0}\right)$ at $400 \mathrm{~nm} \ldots \ldots \ldots \ldots \ldots \ldots \ldots$

6.8 Ionization yield as a function of the internuclear distance. $400 \mathrm{~nm}$ laser with different peak intensities . . . . . . . . . . . . . . . . 50

6.9 Excitation yield as a function of the internuclear distance. $400 \mathrm{~nm}$ laser with different peak intensities . . . . . . . . . . . . . . . 51 


\section{List of Figures}

6.10 Ionization and excitation as a function of peak laser intensity for $\mathrm{H}_{2}(R=$

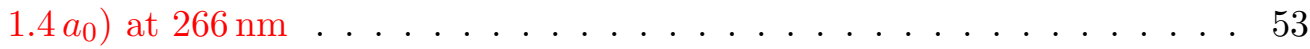

6.11 Ionization yield as a function of the internuclear distance. $266 \mathrm{~nm}$ laser with different peak intensities . . . . . . . . . . . . . . . 54

6.12 Photoelectron spectra for $6 \mathrm{eV}$ photon energy . . . . . . . . . . . . 55

7.1 Ionization yield as a function of incident photon energy for different basis

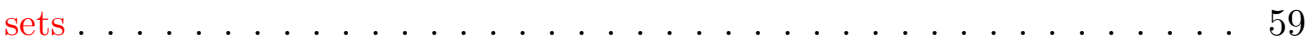

7.2 Excitation yield as a function of incident photon energy for different basis sets . . . . . . . . . . . . . . . . 6 60

7.3 Ionization yield as a function of peak laser intensity at $800 \mathrm{~nm}$ for different

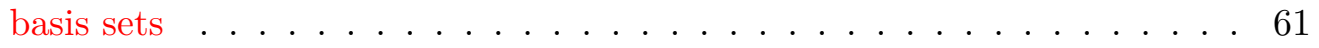

7.4 Ionization and excitation yield as a function of peak laser intensity. Multiplication by 2 for SAE models . . . . . . . . . . . . . . . . 62

7.5 Ionization yield as a function of peak laser intensity for different basis sets

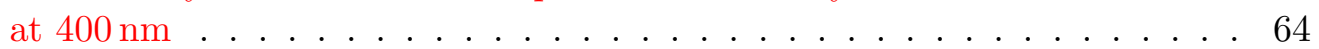

7.6 Excitation yield as a function of peak laser intensity for different basis

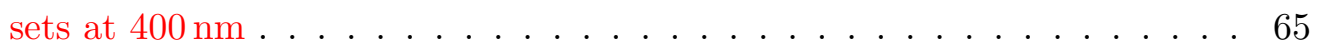

7.7 Ionization yield as a function of peak laser intensity for different basis sets

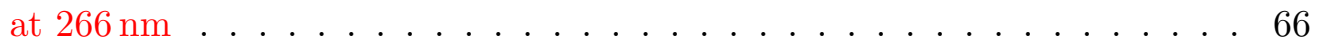

7.8 Excitation yield as a function of peak laser intensity for different basis sets at $266 \mathrm{~nm} \ldots \ldots \ldots \ldots \ldots$

7.9 MO-ADK comparison at $800 \mathrm{~nm} \ldots \ldots \ldots \ldots \ldots$

8.1 Intensity dependence at $2000 \mathrm{~nm}$ for $\mathrm{B}^{1} \Sigma_{u}^{+}$state $\ldots \ldots \ldots \ldots$

8.2 Intensity dependence at $800 \mathrm{~nm}$ for $\mathrm{B}^{1} \Sigma_{u}^{+}$state . . . . . . . . . . . . . 78

8.3 Intensity dependence at $400 \mathrm{~nm}$ for $\mathrm{B}^{1} \Sigma_{u}^{+}$state . . . . . . . . . . . 79

8.4 Single photon ionization regime for $\mathrm{B}^{1} \Sigma_{u}^{+}$state . . . . . . . . . . . . . 80

8.5 Intensity dependence at $8 \mathrm{eV}$ for different pulses . . . . . . . . . . . . 81

8.6 Photoelectron spectra at $8 \mathrm{eV}, \mathrm{B}^{1} \Sigma_{u}^{+}$state as the initial state . . . . . . 83

8.7 Comparison to QSA at $3000 \mathrm{~nm}$ for $\mathrm{B}^{1} \Sigma_{u}^{+}$and $\mathrm{E}, \mathrm{F}^{1} \Sigma_{g}^{+}$states at $R=1.4 a_{0} 84$

9.1 Incident photon energy scan for $\mathrm{O}_{2} \ldots \ldots \ldots$. . . . . . . . . 90

9.2 Incident photon energy scan for $\mathrm{N}_{2} \ldots \ldots \ldots \ldots$. . . . . . . . . 91

9.3 Incident photon energy scan for $\mathrm{C}_{2} \mathrm{H}_{2} \ldots \ldots \ldots \ldots$. . . . . . . . . 92

9.4 Intensity dependence at $800 \mathrm{~nm}$ for $\mathrm{N}_{2}$. MO-ADK and VG-SFA comparison 93

9.5 Intensity dependence at $800 \mathrm{~nm}$ for $\mathrm{O}_{2}$. MO-ADK comparison . . . . . . 94

9.6 Intensity dependence at $800 \mathrm{~nm}$ for $\mathrm{C}_{2} \mathrm{H}_{2} \ldots \ldots \ldots \ldots$

9.7 Intensity dependence at $400 \mathrm{~nm}$ for $\mathrm{N}_{2}, \mathrm{O}_{2}$ and $\mathrm{C}_{2} \mathrm{H}_{2} \ldots \ldots . \ldots 96$ 


\section{List of Tables}

3.1 Ionization potential and $C_{l m}$ values for different basis sets . . . . . . . . 27

8.1 Energies and ionization potential for $\mathrm{B}^{1} \Sigma_{u}^{+}$state . . . . . . . . . . . . 74

8.2 Ionization and ground state population for different basis sets for $8 \mathrm{eV}$ photon energy . . . . . . . . . . . . . . . . . 82 



\section{Selbständigkeitserklärung}

Ich erkläre, dass ich die vorliegende Arbeit selbständig und nur unter Verwendung der angegebenen Literatur und Hilfsmittel angefertigt habe.

Berlin, den 14.12.2009

Manohar Awasthi 